\title{
Scattering of vibrationally excited NO from vanadium dioxide
}

\author{
Dissertation \\ zur Erlangung des mathematisch-naturwissenschaftlichen Doktorgrades \\ "Doctor rerum naturalium" \\ der Georg-August-Universität Göttingen \\ im Promotionsprogramm Chemie \\ der Georg-August University School of Science (GAUSS)
}

\author{
vorgelegt von \\ Artur Meling \\ aus Kaskelen (Kasachstan)
}

Göttingen, 2019 


\section{Betreuungsausschuss}

Prof. Dr. Alec M. Wodtke

Institut für Physikalische Chemie, Georg-August-Universität Göttingen

Prof. Dr. Dirk Schwarzer

Max-Planck-Institut für biophysikalische Chemie, Göttingen

\section{Mitglieder der Prüfungskommission}

Referent: Prof. Dr. Alec M. Wodtke

Institut für Physikalische Chemie, Georg-August-Universität Göttingen

Korreferent: Prof. Dr. Jürgen Troe

Institut für Physikalische Chemie, Georg-August-Universität Göttingen

\section{Weitere Mitglieder der Prüfungskommission}

Prof. Dr. Igor Rahinov

Department of Natural Sciences, The Open University of Israel

Prof. Dr. Martin Suhm

Institut für Physikalische Chemie, Georg-August-Universität Göttingen

Prof. Dr. Dirk Schwarzer

Max-Planck-Institut für biophysikalische Chemie, Göttingen

Dr. Sebastian Kruss

Institut für Physikalische Chemie, Georg-August-Universität Göttingen

Tag der mündlichen Prüfung: 21.01.2020 


\section{Abstract}

This work investigates the surface dynamics of vanadium dioxide with nitric oxide in dependence of its metal-to-insulator transition (MIT) at $68^{\circ} \mathrm{C}$. At lower temperatures $\mathrm{VO}_{2}$ has a monoclinic lattice structure which has low electrical conductance. $\mathrm{VO}_{2}(\mathrm{M})$ is therefore considered an insulator. At higher temperature it is found in its rutile phase, which shows several orders of magnitude more electrical conductance than the monoclinic phase. $\mathrm{VO}_{2}(\mathrm{R})$ is therefore considered a metal.

In previous works it was found that when vibrationally excited NO scatters from metal surfaces, such as $\mathrm{Au}(111)$ and $\mathrm{Ag}(111)$, vibrational energy is transferred via an electron abstraction mechanism to the surface degrees of freedom. It is found that the vibrational relaxation probability of NO scattered from metals is dependent on the difference between the molecule's vertical binding energy at the outer turning point of the vibration and the work function of the surface. This mechanism is not present for scattering from insulator materials such as LiF because they have no electron density at energies similar to the vibrational energy of $\mathrm{NO} . \mathrm{As}_{\mathrm{VO}_{2}}$ is a material which can switch between metal and insulator phase it is investigated how it fits into the previously seen picture of vibrational relaxation of scattered NO.

For this samples of $\mathrm{VO}_{2}$ films are prepared with chemical vapor deposition (CVD) and radio frequency magnetron sputtering (RFMS). The former results in films with grainy surface structure. The latter yields $\mathrm{VO}_{2}$ films with a structure close to single crystals and relatively flat surfaces. Molecular beams of vibrationally excited NO are scattered from various $\mathrm{VO}_{2}$ films and analyzed for their translational, rotational and vibrational excitation after scattering. This is done with time-of-flight spectroscopy and REMPI spectroscopy of the $\gamma$-band system of NO.

It is shown that $\operatorname{NO}(v=2, J=0.5)$ and $\mathrm{NO}(v=11, J=0.5)$ scattered from CVD prepared films are dominantly scattered via the direct scattering channel. Scattered NO molecules retain mostly their initial vibrational state during scattering and have a hyperthermal rotational excitation after scattering. Furthermore, the translational energy after scattering and the rotational excitation increase both with the translational energy of the incident molecular beam. During the experiments no trapping desorption is observed but a certain amount of NO is seen with lower rotational and vibrational 
excitation and lower translational energy than the directly scattered molecules. It is assumed that they penetrate into the gaps between the $\mathrm{VO}_{2}$ grains of the CVD prepared surface and bounce multiple times before they leave the surface again. For NO scattered from CVD prepared $\mathrm{VO}_{2}$ no clear sign of a increased vibrational relaxation probability of $\mathrm{NO}$ scattered from $\mathrm{VO}_{2}(\mathrm{R})$ compared to $\mathrm{VO}_{2}(\mathrm{M})$ could be observed.

This is attributed to the fact that the CVD prepared samples are cleaned with $\mathrm{Ar}^{+}-$ ion sputtering which is found to eliminate oxygen from the $\mathrm{VO}_{2}$ film. This cleaning technique is yet not able to remove all carbon contaminations present on the surface of CVD prepared $\mathrm{VO}_{2}$. The former results in a changed chemical composition of the $\mathrm{VO}_{2}$ film, which causes a reduced contrast of the MIT. The latter results in surfaces partially covered in carbon. In consequence, it can not be outruled that a certain amount of NO scatters from carbon instead of $\mathrm{VO}_{2}$. To improve the surface cleaning procedure this work also investigates the application of $\mathrm{O}_{2}{ }^{+}$-ion sputtering. It is shown that $\mathrm{O}_{2}{ }^{+}$-ion sputtering is significantly more efficient in removing carbon contaminations from the surface. Furthermore, it negates the effect of oxygen elimination from the $\mathrm{VO}_{2}$ film by inserting oxygen back into the surface. This retains the chemical composition of $\mathrm{VO}_{2}$ during sputtering and in consequence also the contrast of the MIT. The $\mathrm{O}_{2}{ }^{+}$-ion sputtering technique is applied to the later RFMS prepared $\mathrm{VO}_{2}$ thin films instead of $\mathrm{Ar}^{+}$-ion sputtering.

$\mathrm{NO}(v=3, J=0.5)$ is prepared in a molecular beam with a translational energy of $E_{\text {inc,trans }}=0.99 \mathrm{eV}$ and scattered from RFMS prepared $\mathrm{VO}_{2}$ thin films. The directly scattered molecules relax with a probability of $2 \%$ to the $(v=2)$ state when scattered from $\mathrm{VO}_{2}(\mathrm{M})$. The vibrational relaxation probability increases by $0.3 \%$ when the thin film changes to $\mathrm{VO}_{2}(\mathrm{R})$ at $68^{\circ} \mathrm{C}$. No population is found for $\mathrm{NO}(v=1)$ for both phases of $\mathrm{VO}_{2} . \mathrm{NO}(v=11, J=0.5)$ is prepared in molecular beams with translational energies of $E_{\text {inc,trans }}=0.92 \mathrm{eV}, E_{\text {inc,trans }}=0.73 \mathrm{eV}$ and $E_{\text {inc,trans }}=0.47 \mathrm{eV}$. For direct scattering from RFMS prepared $\mathrm{VO}_{2}(\mathrm{M})$ vibrational relaxation probabilities of about $20 \%$ are found which increase with the translational energy of the incident molecular beam. For $\mathrm{VO}_{2}(\mathrm{R})$ the relaxation probability increases by $3 \%$ compared to scattering from $\mathrm{VO}_{2}(\mathrm{M})$.

A detailed comparison with the vibrational relaxation probabilities found for NO scattered from $\mathrm{Ag}(111)$ and $\mathrm{Au}(111)$ is done to understand why relaxation of $\mathrm{NO}$ from $\mathrm{VO}_{2}$ is significantly less likely than from other metals. For this the image charge stabilization, work function and the charge carrier density of $\mathrm{VO}_{2}(\mathrm{M}), \mathrm{VO}_{2}(\mathrm{R}), \mathrm{Au}(111)$ and $\operatorname{Ag}(111)$ are compared.

This work also shows the comparison of $\mathrm{NO}(v=11)$ scattered from $\mathrm{SiO}_{2}$ and $\mathrm{VO}_{2}$. It is shown that for scattering from $\mathrm{SiO}_{2} \mathrm{NO}$ is only found in the vibrational states 
$v=11$ and $v=10$. For scattering from $\mathrm{VO}_{2}$ additionally a small amount of population is observed in the vibrational states $4<v<10$.

Rotational state distributions of scattered $\mathrm{NO}(v=3)$ and $\mathrm{NO}(v=2)$ from the scattering experiments of $\mathrm{NO}(v=3, J=0.5)$ from RFMS prepared $\mathrm{VO}_{2}$ thin films are analyzed. It is shown that the rotational energy of scattered $\mathrm{NO}(v=3)$ is significantly higher that the rotational excitation of thermal $\mathrm{NO}(v=3)$ at the surface temperature. The rotational excitation of the vibrationally relaxed $\mathrm{NO}(v=2)$ is more than $50 \%$ higher than the rotational excitation of $\mathrm{NO}(v=3)$. The same effect for the rotational excitation of $\mathrm{NO}(v=11)$ and $\mathrm{NO}(v=10)$ is also shown in the scattering experiments of $\mathrm{NO}(v=11, J=0.5)$ scattered from RFMS prepared $\mathrm{VO}_{2}$ thin films and for $\mathrm{NO}(v=11, J=0.5)$ scattered from $\mathrm{SiO}_{2}$. This suggests that a mechanism is present during the scattering which transfers vibrational energy to the rotational quanta which has yet not been reported for NO scattering from metal surfaces. 



\section{Contents}

$\begin{array}{lll}1 & \text { Introduction } & 1\end{array}$

\begin{tabular}{|lll}
2 & Theoretical Background & $\mathbf{5}$
\end{tabular}

$2.1 \quad$ Metal-to-Insulator Transitions in Vanadium Oxides . . . . . . . . . . . . 5

$2.2 \quad$ Resonance Enhanced Multi Photon Ionization Spectroscopy of Nitric Oxide 11

2.2 .1 Quantum States of Diatomic Molecules . . . . . . . . . . . . 11

2.2 .2 Hund's Cases . . . . . . . . . . . . . . . . . . . . . . . . . 14

$2.2 .3 \quad$ Quantum States of Nitric Oxide $\ldots \ldots \ldots \ldots$

2.3 Surface Scattering with Nitric Oxide . . . . . . . . . . . . . . . . . 21

$2.3 .1 \quad$ Born-Oppenheimer Approximation and Breakdown . . . . . . . . 22

$2.3 .2 \quad$ Scattering of vibrationally excited NO . . . . . . . . . . . . 28

$\begin{array}{lll}3 & \text { Experimental Setup } & \mathbf{3 5}\end{array}$

3.1 Home-Built UHV Compatible Four-Terminal Sensing Device. . . . . . . 35

3.2 Preparation of $\mathrm{VO}_{2}$ films $\ldots \ldots \ldots \ldots \ldots \ldots \ldots$

$3.2 .1 \quad$ Chemical Vapor Deposition . . . . . . . . . . . . . . . . . . 36

$3.2 .2 \quad$ Reactive Radio Frequency Magnetron Sputtering . . . . . . . . . 39

3.2 .3 Preparation of $\mathrm{VO}_{2}$ under UHV conditions for Surface Scattering 39

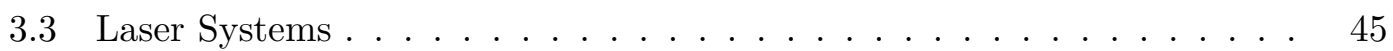

3.3.1 Preparation of $\mathrm{NO}$ in $\mathrm{X}^{2} \Pi(v=3) \ldots \ldots \ldots \ldots$. . . . . . . 45

$3.3 .2 \quad 1+1^{\prime}$ REMPI of $\mathrm{NO}$ in $\mathrm{X}^{2} \Pi(v=3) \ldots \ldots \ldots . \ldots . \ldots 47$

$3.3 .3 \quad$ Preparation of $\mathrm{NO}$ in $\mathrm{X}^{2} \Pi(v=11) \ldots \ldots \ldots . \ldots . \ldots 47$

$3.3 .4 \quad 1+1$ REMPI of NO in $\mathrm{X}^{2} \Pi(v=11) \ldots \ldots \ldots$

3.4 Molecular Beam Apparatus . . . . . . . . . . . . . . . . . . . . . . 49

$3.4 .1 \quad$ Apparatus for Surface Scattering of $\mathrm{NO} \mathrm{X}^{2} \Pi(v=3) \quad \ldots . . \quad 49$

$3.4 .2 \quad$ Apparatus for Surface Scattering of $\mathrm{NO} \mathrm{X}^{2} \Pi(v=11) \ldots \ldots . . .51$

3.5 Experimental Procedures . . . . . . . . . . . . . . . . . 53

3.5 .1 Timings and MCP detector . . . . . . . . . . . . . . 53

3.5 .2 Time-of-Flight Analysis . . . . . . . . . . . . . . . . . 55

$\begin{array}{lll}3.5 .3 & \text { REMPI Spectrum Analysis }\end{array} \ldots \ldots \ldots$. . . . . . . . . . . . 58

3.5.4 Angular Distribution Procedure . . . . . . . . . . . . . . . . 62 
4 Scattering of NO from CVD prepared $\mathrm{VO}_{2}$ films 65

$4.1 \quad$ Scattering of $\mathrm{NO}(v=0)$ and $\mathrm{NO}(v=11)$ from CVD1 and CVD2 . . . . 65

$4.1 .1 \quad$ Samples Characteristics of CVD1 and CVD2 . . . . . . . . 65

$4.1 .2 \quad$ Angular Distribution of Scattered NO . . . . . . . . . . . . 67

$4.1 .3 \quad$ Time-of-Flight Distributions of Scattered NO . . . . . . . . . . . 68

$4.1 .4 \quad$ REMPI Spectra of Scattered NO . . . . . . . . . . . . . . . 74

$4.2 \quad$ Scattering of $\mathrm{NO}(v=2)$ from CVD3 $\ldots \ldots \ldots \ldots \ldots . \ldots \ldots$

$4.2 .1 \quad$ Sample Characteristics of CVD3 $\ldots \ldots \ldots \ldots \ldots$

4.2 .2 Time-of-Flight Spectroscopy of Scattered NO . . . . . . . . . . . 80

$4.2 .3 \quad$ REMPI Spectra of Scattered NO . . . . . . . . . . . . . . . . . 82

4.3 Discussion $\ldots \ldots \ldots \ldots \ldots \ldots$. . . . . . . . . . . . . . . . . . . . 87

5 Scattering of $\mathrm{NO}(v=11)$ from Silicon Dioxide $\quad 89$

5.1 Sample Characterization . . . . . . . . . . . . . . . . . . . 89

$5.2 \quad$ Angular Distribution of Scattered NO . . . . . . . . . . . . . . . 90

5.3 Time-of-Flight Spectroscopy of Scattered NO . . . . . . . . . . . . . . . 90

5.4 REMPI Spectra of Scattered NO $\ldots \ldots \ldots$. . . . . . . . . . . . . 92

5.5 Discussion $\ldots \ldots \ldots \ldots \ldots \ldots$. . . . . . . . . . . . . . . . . . . . 94

$\begin{array}{lll}6 & \text { Scattering of NO from RFMS prepared } \mathrm{VO}_{2} \text { thin films } & 97\end{array}$

$6.1 \quad$ Scattering of $\mathrm{NO}(v=3)$ from RFMS1 and RFMS2 . . . . . . . . . . . . 97

$6.1 .1 \quad$ Sample Characterization of RFMS1 and RFMS2 . . . . . . . . . . . 97

$6.1 .2 \quad$ Angular Distribution of Scattered NO . . . . . . . . . . . 100

$6.1 .3 \quad$ Time-of-Flight Spectroscopy of Scattered NO . . . . . . . . . . . 100

$6.1 .4 \quad$ REMPI Spectra of Scattered NO . . . . . . . . . . . . . . . . 104

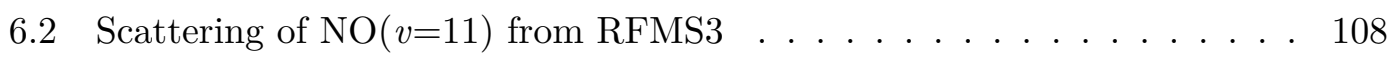

$6.2 .1 \quad$ Sample Characterization of RFMS3 $\ldots \ldots \ldots$

$6.2 .2 \quad$ Angular Distributions of Scattered NO . . . . . . . . . . . . . 110

6.2 .3 Time-of-Flight Spectroscopy of Scattered NO . . . . . . . . . . . 111

$6.2 .4 \quad$ REMPI Spectra of Scattered NO . . . . . . . . . . . . . . . 113

6.3 Discussion $\ldots \ldots \ldots \ldots \ldots$

$6.3 .1 \quad$ Low Relaxation Probability of Scattered NO . . . . . . . . . . 118

$6.3 .2 \quad$ Elevated Rotational Temperatures in Relaxed NO . . . . . . . . 123

\begin{tabular}{lll}
\hline & Summary and Outlook & 125
\end{tabular}

\begin{tabular}{|ll}
\hline A Appendix & 129
\end{tabular}

A.1 Custom-made LABView Environment . . . . . . . . . . . . . . . . . . . 129 
Contents

\begin{tabular}{ll}
\hline List of Abbrevations and Indices & 135
\end{tabular}

\begin{tabular}{ll}
\hline Bibliography & 137
\end{tabular}

\begin{tabular}{ll}
\hline Acknowledgments & 145
\end{tabular} 



\section{Introduction}

This work is dedicated to broaden the understanding of molecule-surface dynamics on a fundamental level. The relevance of these dynamics becomes clear when one understands its connection to heterogeneous catalysis.

Heterogeneous catalysis is a technique used to reduce drastically the energetic barrier of a specific chemical reaction step. In simple words, intermediates of chemical reactions are stabilized through adsorption onto solid materials, the heterogeneous catalysts, present during the reaction process 1 . In consequence, less energy has to be brought up to form these intermediate species resulting in higher reaction rates and lower energy consumption. Heterogeneous catalysts are used on an industrial scale since the late 18th century. The first industrial application of a heterogeneous catalyst was in the "contact process" used by the german company "Badische Anilin und Soda-Fabrik" 2]. Originally, a platinum catalyst is used to oxidize sulfur dioxide with oxygen to sulfur trioxide which is then used to form sulfuric acid. To this day sulfuric acid is one of the most important chemicals with a production of 180 megatonnes in $2004^{3}$, though nowadays vanadium pentoxide is used instead of platinum.

Another often cited application of heterogeneous catalysis is the Haber-Bosch process used for the synthesis of ammonia from atmospheric nitrogen with a magnetite $\left(\mathrm{Fe}_{3} \mathrm{O}_{4}\right)$ catalyst ${ }^{4}$. The Haber-Bosch process changed the world like no other invention since it allowed the production of synthetic fertilizer. This led to a unprecedented growth of the world population with all its consequences ${ }^{[5}$, which would otherwise never been possible.

The high prevalence of industrial chemicals in our daily life makes it important to find new catalyst to increase the efficiency of industrial processes. New technologies can help to decrease power requirements of chemical plants and are of course also financially interesting. Therefore, large amounts of time, man power and money are invested into the research of heterogeneous catalysts. Despite these factors catalyst research is to this day mainly conducted with a trial and error approach where various substances are tested for their catalytic activity. Of course, this is very time-consuming but often the only available way to improve the already used catalysts since the detailed reac- 
tion mechanism is not known and can often not be probed directly during the reaction, making many catalytic reactions basically black boxes.

The goal of the so-called "surface science" discipline of physical chemistry is to shed light into these catalyst reactions. It approaches the real life chemical reactions with model systems of catalysts and reactants to investigate the basic principles along which the chemical mechanisms work. Irving Langmuir can be regarded as the forefather of surface science. He published his work on the chemisorption of gas molecules on metal surfaces around the time of World War I and showed how it can be used to formulate rate laws for the elemental reactions that occur on the surface ${ }^{6}$. In the following decades advances in spectroscopic and structural measurement techniques allowed to further investigate these elemental reactions ${ }^{2}$.

This all culminates in the use of molecular beam scattering in ultra high vacuum apparatus from atomically flat surfaces with precisely cut surfaces as it is done on several experimental setups in the Wodtke group. This way all interfering influences can be excluded and only the reactions of interest are probed, which can lead to new discoveries on reactions which were formally regarded as well understood. One example is the oxidation of carbon monoxide on a platinum surface ${ }^{7}$. Through the use of an imaging detector it was possible to map the correct reaction mechanism and rate constants which could previously not be probed $\stackrel{8}{\text {. }}$.

Earlier than this, the role of vibrational motion in the electron transfer from a surface to the molecule was found by applying the above mentioned surface science methods $\underline{9}$. This was followed by a row of similar experiments ${ }^{10}$, which among other things investigated the role of $\mathrm{NO}$ orientation during scattering 11 and the difference between $\mathrm{Au}(111)$ and $\mathrm{Ag}(111)$ surfaces stemming from the different work function 12 . The role of vibrational excitation and electron affinity was probed by repeating the scattering experiments with vibrationally excited $\mathrm{CO} 13$ 14. This all culminates in the general picture of vibrational relaxation probability in dependence of the difference between work function of the surface and vertical electron binding energy at the outer turning point of the molecular vibrational motion published by Steinsiek in $2018^{15}$.

As already stated above all these experiments use mostly noble metals with atomically flat single crystal surfaces as models for heterogeneous catalysts. While this all led to new discoveries and advanced theoretic models which can be used to better describe catalytic reactions, they are still far away from real life catalytic processes. So it might be fruitful to use the tried and tested surface science techniques and experiments on a surface which resembles in its structure more actual used catalysts and is also composed of a substance which has already proven catalytic activity. Vanadium dioxide $\left(\mathrm{VO}_{2}\right)$ fulfills these criteria as it can be prepared with various deposition techniques as a film. 
Also the related compound vanadium pentoxide is already used as an industrial catalyst in the contact process for the synthesis of sulfur trioxide ${ }^{3}$ and for the selective reduction of $\mathrm{NO}_{\mathrm{x}}{ }^{16}$. Another point that makes $\mathrm{VO}_{2}$ an interesting substance for surface science experiments is its intense metal-to-insulator (MIT) transition. At $68^{\circ} \mathrm{C}$ it undergoes a phase transition from a monoclinic to a rutile crystal structure where the resistance of the solid changes by up to 5 orders of magnitude ${ }^{17}$. Since its discovery by Morin in the 1950s ${ }^{18}, \mathrm{VO}_{2}$ has been heavily investigated because of this bulk property. This work investigates how molecular beam scattering with vibrationally excited $\mathrm{NO}$ can be conducted from rough CVD prepared $\mathrm{VO}_{2}$ films. Furthermore, it shows how the MIT of radio frequency magnetron sputtering prepared thin film $\mathrm{VO}_{2}$ with a more single crystal like structure influences the relaxation probability of scattered $\operatorname{NO}(v=3)$ and $\mathrm{NO}(v=11)$. The key objective of this work is to conduct a comprehensive series of experiments where the influence of electron-hole pairs (EHP) excitation can be disentangled from other mechanisms of energy dissipation, yielding quantitative evaluation of the EHP-vibration coupling role in heterogeneous catalysis. A central aim of this work was to carry out state-to-state molecular beam surface scattering experiment in using solid-state $\mathrm{VO}_{2}$ samples that are realistic models for modern catalysts. The experiments have employed scattering of laser-prepared molecular beams of NO molecules from well characterized $\mathrm{VO}_{2}$ film samples. In these experiments the availability of EHP states will be varied in a controlled fashion by temperature variation in the vicinity of the MIT for the $\mathrm{VO}_{2}$ films. The propensity of EHP-vibration coupling was quantified via quantum state-specific, velocity and angular-resolved laser based detection of the nascent products. Such experiments are capable to generate data revealing the underlying dynamics. The dependence of metallic properties of $\mathrm{VO}_{2}$ films on their temperature should clearly bear on their propensity to interact with molecular vibrational coordinate and hence, possibly influence the chemistry of heterogeneous catalysis. To date these aspects have never been examined in well-controlled dynamical experiments and were, for the first time, addressed in the present work. The results are put into the context of the relaxation probability plot suggested by Krüger, Golibrzuch, Steinsiek and Wagner and compared to the results of NO scattering from $\mathrm{Au}(111)$ and $\mathrm{Ag}(111)$. 



\section{Theoretical Background}

\subsection{Metal-to-Insulator Transitions in Vanadium Oxides}

This work discusses the interaction of gaseous NO molecules when scattered from vanadium dioxide $\left(\mathrm{VO}_{2}\right)$ surfaces. This material is most famous for its pronounced metal-toinsulator transition (MIT). Because of this $\mathrm{VO}_{2}$ and its possible applications have been studied intensely 17 [19 since their discovery in the 1950s by Morin 18 . This section will explain the general properties of MIT materials and the special characteristics of $\mathrm{VO}_{2}$ as a basis to understand the relevance of MIT materials for surface dynamics.

Solid materials exist in two main categories, metals and insulators. These two differ by the fact that electrons can move relatively freely through a metal while they experience considerable resistance in an insulator. This results in metals showing higher electrical and thermal conductance than insulators and often a metallic shine. Insulators do not conduct electricity and are often used to isolate electrical charges. This phenomenon is explained by the band model ${ }^{20}$. When atom orbitals of similar energy are forced into close proximity they start two perturb with each other. This results in two new orbitals which are shifted energetically up and down with respect to the energy of the initial atom orbitals. The concept is shown schematically in Figure 2.1. This perturbation also occurs for higher numbers of atoms in a lattice where with each additional atom the energetic distance between two neighboring states is reduced until the perturbed lattice orbitals form a quasi-continuous energy band. The difference between a metal and an insulator in the band model is that the Fermi level of a metal is located inside of a band, while the Fermi level of an insulator is located in between two bands in a so-called band gap as shown in the first three cases depicted in Figure 2.2. Therefore, in a metal the lowest occupied band, called valence band, is never completely filled under standard conditions. So that electrons can excite to the next unoccupied electronic state with minimal energy expenditure. This way the electron can move through the solid. For insulators the lowest occupied band is always completely filled so that the next available unoccupied states the electrons can move to are across the band gap which can be several $\mathrm{eV}$ wide. Therefore, in this case electrons are under standard conditions bound to the valence band and can only be moved with high energy expenditure. Semiconduc- 


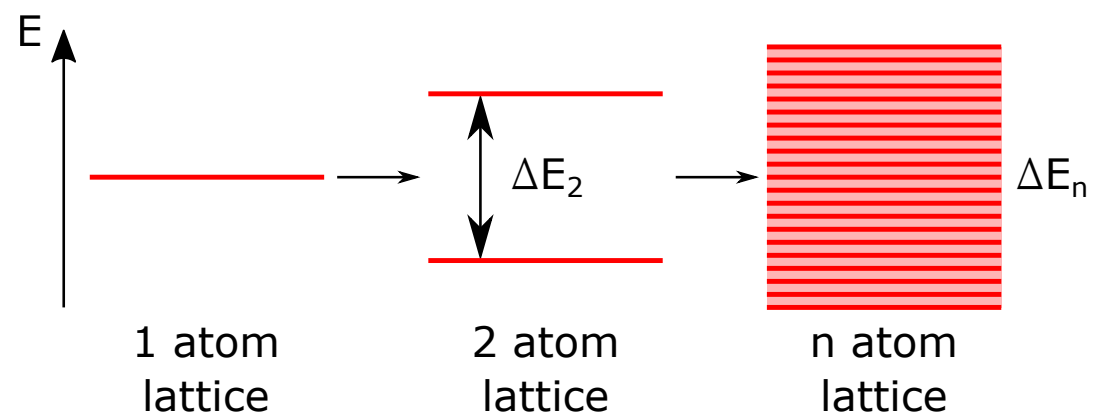

Figure 2.1: Schematic depiction of the energy of a single atom orbital in contrast to two and $n$ perturbed atom orbitals in a hypothetical crystal lattice. For the energy differences between the states of the two atom lattice $\Delta E_{2}$ and the $\Delta E_{n}$ atom lattice $\Delta E_{2} \gg \Delta E_{n}$ applies.

tors can be considered as a special case of insulator where the band gap is sufficiently small so that electron excitation can occur with low energetic effort. The upper band gap is in this case called conduction band where the excited electron can move freely like in a metal, which results in drastically increased electrical conductance. This can be achieved by light stimulation, heat, pressure or electrical discharge. A schematic depiction of the band structure of a semi-conductor is shown in the last case of Figure 2.2 ,

Most materials can be described by this model. In 1937 it was reported that many transition metal oxides with partially filled d-electron bands do show very low conductance, although they should be metals according to the band model 21 . This phenomenon was intensely investigated by Nevill F. Mott for over 60 years and materials with such a behavior are usually referred to as Mott Hubbard or more often just Mott insulators $\underline{22}$. Mott and Peierls $\frac{23}{23}$ attributed the non-conductivity of these crystals to electron-electron interactions, namely strong Coulomb repulsion between electrons.

If we imagine a crystal lattice of $n$ equal atoms where each atom contributes a single atom orbital and electron, we get an energy splitting as shown in Figure 2.1. In the simple model the electronic ground state of the lattice consists of electrons filling up all the lower lattice orbitals and leave the energetically higher lattice orbitals empty. One can imagine the ground state as a situation where on half of the orbitals is occupied by always two electrons, while the rest is left empty. By excitation with relatively low energy electrons can be moved to the unoccupied upper lattice orbitals. This way they can jump from orbital to orbital and along the crystal lattice. This is shown schematically on the left side of Figure 2.3. On the macroscopic scale the solid becomes electrically conducting and can be classified as a metal.

But this picture neglects the repulsive Coulomb-interaction between the electrons. This 


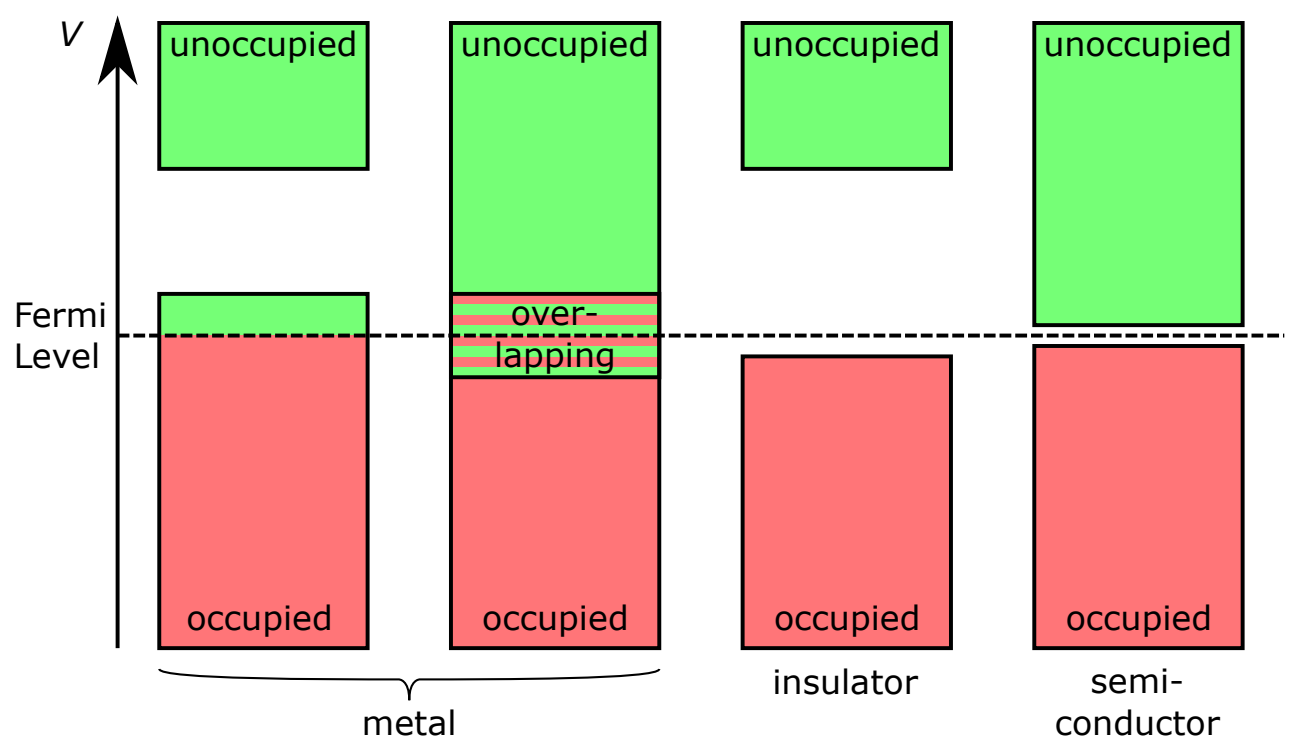

Figure 2.2: Band Model of a structure where the highest occupied band is partially occupied (metal), where the highest occupied band overlaps with the lowest unoccupied band forming a partially occupied composite band (metal) and a structures with a large band gap between highest occupied band and the lowest unoccupied band (insulator) and a similar structure with a small band gap (semiconductors).

is a force that pushes the electrons away from each another. This makes it energetically unfavorable to place two of them in the same orbital or atom. If the Coulomb repulsion is stronger than the energy gap between the lattice orbitals it is energetically favorable for the lattice to place all electrons in individual orbitals. In this case electron motion is only possible if enough energy is expended to force the electrons into an already partially occupied orbital. This is shown schematically on the right side of Figure 2.3. This situation resembles an insulator where a band gap has to be crossed to put the electrons in a position where they can gain mobility. Therefore, we have a material that while it has every prerequisite of a metal it does not conduct electricity.

Another cause for the low electrical conductance of Mott insulators is postulated by Slater 24. Through anti-ferromagnetic long-range order the electrons are forced into certain lattice positions. To move electrons in this material the magnetic ordering force has to be overcome. This results in the same situation as shown on the right side of Figure 2.3 .

The conductance is only suppressed as long as the forces, that keep the electrons in place, are prevailing. For the case, where Coulomb repulsion is the cause of the Mott insulator, subtle changes in the crystal structure that shift the energetic structure can diminish the Coulomb repulsion and consequently the band gap. This can be for ex- 


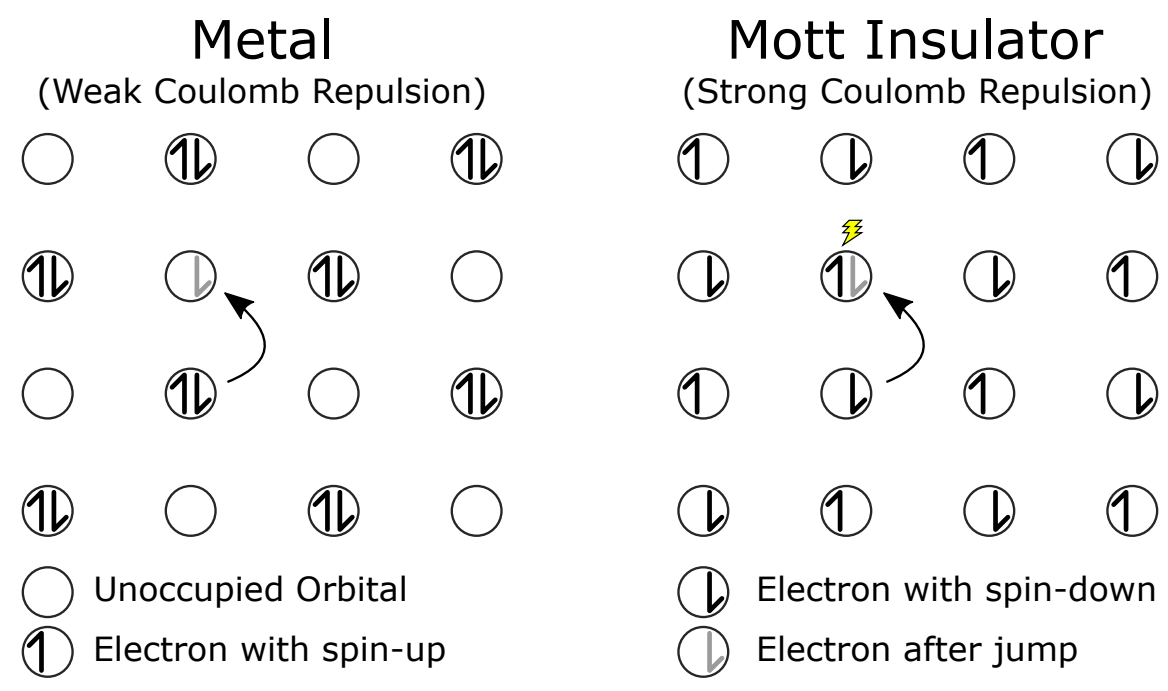

Figure 2.3: Schematic depiction of the electron distribution over the lattice orbitals for a metal (with weak Coulomb repulsion force) and a Mott insulator (with strong Coulomb repulsion force). In the metal the electrons occupy as few orbitals as possible while in the Mott insulator they are evenly distributed over all available orbitals. In the metal the electrons can move by jumping from orbital to orbital with a very low energetic barrier in between. In the Mott insulator the Coulomb repulsion has to be overcome which hinders the movement significantly. Please note that in reality the electrons of a metal form a delocalized electron cloud over the crystal. The depiction of localized electrons above is chosen deliberately to emphasize the difference between metal and mott insulator.

ample a phase transition or deformation of the crystal lattice by pressure. If the Mott insulator is caused by magnetic ordering the conductance of the material increases significantly once the Néel temperature is reached. When these requirements are met the conductivity of the solid can increase by several orders of magnitude while the outer appearance of it remains the same. Such transitions are therefore referred to as metalto-insulator transitions (MIT).

Another cause for a hindered electron mobility can be the very structure of a lattice. When the otherwise metallic parts, which can be atoms or larger clusters of atoms, are isolated from each other by distances over which electrons can not be exchanged, the same situation as with a Mott insulator is found. During phase transitions, where the lattice particles are rearranged so that these isolated islands are reconnected, the electrons can suddenly move freely over the whole crystal and the conductivity increases drastically. In this case one speaks of a Peierls transition or Peierls insulator. 


\section{Transition from Monoclinic to Rutile Vanadium Dioxide}

In most reports about MITs vanadium oxides are prominently featured since many of these oxides exhibit a MIT at various temperatures with partially high contrast. They can be grouped in two categories. On one hand there are Magnéli-type vanadium oxides ${ }^{25]}$ with the general formula $\mathrm{V}_{n} \mathrm{O}_{2 n-1}$. Examples of the Magnéli-type vanadium oxides are shown in Table 2.1. All these substances are Mott insulators through magnetic ordering except $\mathrm{V}_{7} \mathrm{O}_{13}$ which is always metallic.

Table 2.1: Examples of the $\mathrm{V}_{n} \mathrm{O}_{2 n-1}$ Magnéli-type vanadium oxides. Table data taken from Reference [26].

\begin{tabular}{ccc}
\hline$n$ & Molecular formula & MIT temperature $T_{\text {MIT }}[\mathrm{K}]$ \\
\hline 2 & $\mathrm{~V}_{2} \mathrm{O}_{3}$ & 168 \\
3 & $\mathrm{~V}_{3} \mathrm{O}_{5}$ & 430 \\
4 & $\mathrm{~V}_{4} \mathrm{O}_{7}$ & 250 \\
5 & $\mathrm{~V}_{5} \mathrm{O}_{9}$ & 135 \\
6 & $\mathrm{~V}_{6} \mathrm{O}_{11}$ & 170 \\
8 & $\mathrm{~V}_{8} \mathrm{O}_{15}$ & 70 \\
\hline
\end{tabular}

On the other hand there are vanadium oxides with the general formula $\mathrm{V}_{n} \mathrm{O}_{2 n+1}$. Examples of this kind of vanadium oxide are listed in Table 2.2

Table 2.2: Examples of the $\mathrm{V}_{n} \mathrm{O}_{2 n+1}$ type vanadium oxides.

\begin{tabular}{cccc}
\hline$n$ & Molecular formula & MIT temperature $T_{\mathrm{MIT}}[\mathrm{K}]$ & Reference \\
\hline 2 & $\mathrm{~V}_{2} \mathrm{O}_{5}$ & 550 & {$[27]$} \\
3 & $\mathrm{~V}_{3} \mathrm{O}_{7}$ & 5.2 & {$[28]$} \\
6 & $\mathrm{~V}_{6} \mathrm{O}_{13}$ & 154 & {$[29]$} \\
\hline
\end{tabular}

$\mathrm{VO}_{2}$ can be counted to both types of vanadium oxides if one assumes $n=\infty$. It exhibits a fully reversible MIT at $341 \mathrm{~K}^{17]}$ where the conductivity changes by up to 5 orders of magnitude. Furthermore, the transition temperature can be reduced by doping the crystal with tungsten ${ }^{30}$ or molybdenum 31 . Besides its electric properties $\mathrm{VO}_{2}$ also undergoes a measurable change in IR reflectivity along the MIT ${ }^{32}$. The combination 


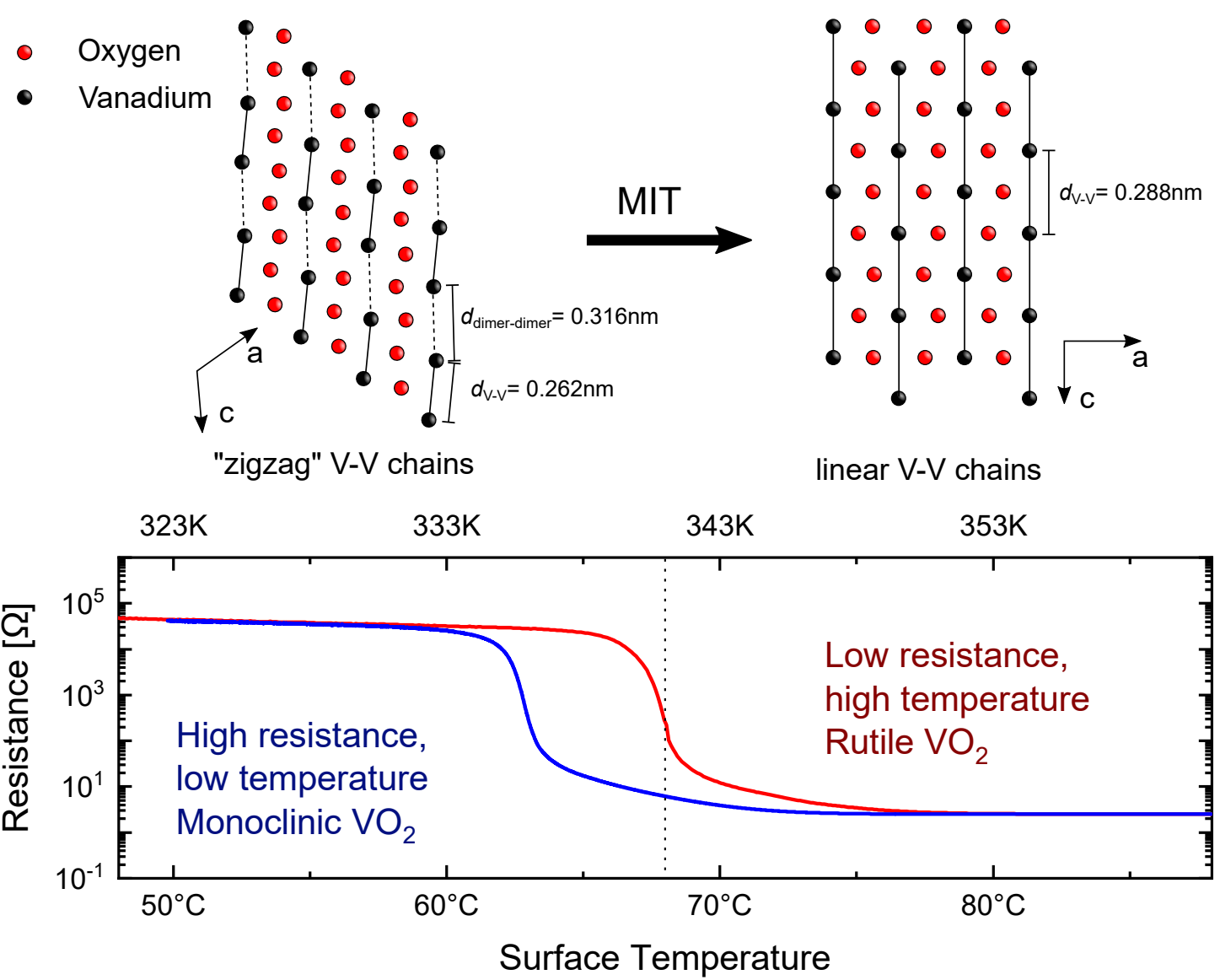

Figure 2.4: Change of the lattice structure of monoclinic $\mathrm{VO}_{2}(\mathrm{M})$ to rutile $\mathrm{VO}_{2}(\mathrm{R})$ along the temperature induced $\mathrm{MIT}$ at $68^{\circ} \mathrm{C}$ (pointed line). In $\mathrm{VO}_{2}(\mathrm{M})$ the vanadium atoms form "zigzaging" lines along the crystal lattice. Two vanadium atoms each form a dimer with a distance of $d_{\mathrm{V}-\mathrm{V}}=0.262 \mathrm{~nm}$ between its atoms and $d_{\text {dimer-dimer }}=0.316 \mathrm{~nm}$ between two dimers. In $\mathrm{VO}_{2}(\mathrm{R})$ the vanadium atoms line up to straight lines with equal distances of $d_{\mathrm{V}-\mathrm{V}}=0.288 \mathrm{~nm}$ between atoms. Since the critical distance to exchange electrons for vanadium is $d_{\text {crit }}=0.294 \mathrm{~nm}$, electric conductance is only possible in $\mathrm{VO}_{2}(\mathrm{R})$. Adapted from Reference [17.

of these properties makes $\mathrm{VO}_{2}$ a likely candidate for room temperature applications like Mott transistors $\underline{33}$ or smart windows $\underline{34}$. Although $\mathrm{VO}_{2}$ is one of the first described and investigated MIT materials ${ }^{36}$ it is still discussed if it can actually be classified as a Mott insulator or a Peierls insulator. More recent investigations suggest that the latter to be the case.

In Reference [17] the structural transition of vanadium dioxide and its influence on the conductivity and IR reflectivity along the temperature is described in detail. A schematic of the structural transition is shown in Figure 2.4. In short, the transition is 
explained by the fact that in monoclinic vanadium dioxide the vanadium atoms form socalled zigzagging chains along the crystal consisting of V-dimers with a V-V distance of

$d_{\mathrm{V}-\mathrm{V}}=2.62 \AA$ and a interdimer distance of $d_{\text {dimer-dimer }}=3.16 \AA$. As the critical distance for $\mathrm{V}-\mathrm{V}$ electronic interaction is $d_{\text {crit }}=2.94 \AA$ the electrons are trapped inside of the dimer and cannot move along the crystal. Therefore, although the electrons are located in principle in a partially unoccupied band, as it is usual for a metal, their movement is hindered by a potential hill reminiscent of a band gap. During the MIT the lattice rearranges to rutile $\mathrm{VO}_{2}$ where the vanadium atoms form straight lines with a uniform $\mathrm{V}-\mathrm{V}$ distance of $d_{\mathrm{V}-\mathrm{V}}=2.88 \AA$. In this case the $\mathrm{V}-\mathrm{V}$ distance is below the critical interaction distance and the electrons can be exchanged freely between the atoms. This leads to a drastically increased conductivity of the crystal.

\subsection{Resonance Enhanced Multi Photon Ionization Spectroscopy of Nitric Oxide}

This work is mostly concerned with the rovibrational excitation of Nitric Oxide (NO) scattered from $\mathrm{VO}_{2}$. It is used to probe the interaction of molecular quantum states with surface electrons on a microscopic scale. For this the properties of scattered NO are probed with Resonance Enhanced Multi Photon Ionization (REMPI) spectroscopy through excitation with high intensity pulsed laser beams. The following section will give a description of the quantum states of nitric oxide which are probed using REMPI spectroscopy and the selection rules which govern the shape of the REMPI spectra.

\subsubsection{Quantum States of Diatomic Molecules}

To understand REMPI spectroscopy of diatomic molecules one has to first understand the quantum structure of this kind of particles, their properties and the nomenclature necessary to describe them. The following section is based on the descriptions in the book by Herzberg "Spectra of diatomic molecules" 37 .

In atoms the motion of electrons is restricted to a force field of spherical symmetry around the nucleus. Its extend of this electronic motion is described sufficiently well by the constant electronic orbital angular momentum vector $\vec{L}$ as long as we neglect the influence of the electron's spin. Combining two atoms to a molecule reduces the symmetry of the force field to axial symmetry about the internuclear axis. Similar to an atom in an electric field the electronic orbital angular momentum vector $\vec{L}$ is also bound to the electric field generated by electrostatic potentials between the atoms. The direction of this field is for obvious reasons always along the internuclear axis so that 
the procession of $\vec{L}$ occurs always around the internuclear axis. The only remaining constant component of $\vec{L}$ is the projection onto the axis $h / 2 \pi \vec{M}_{\mathrm{L}}$ as shown in Figure 2.5. The precession rate becomes faster with a stronger electric field so that $\vec{L}$ becomes increasingly ill defined with $\vec{M}_{\mathrm{L}}$ remaining as the only good quantum number of the electronic orbital angular momentum. For this reason, $\vec{M}_{\mathrm{L}}$ is used instead of $\vec{L}$ to describe the electron motion. Since the possible directions of $\vec{L}$ are quantizised the same is true for the length of $\vec{M}_{\mathrm{L}}$ which can only appear with the numbers

$$
\vec{M}_{\mathrm{L}}=L, L-1, L-2, \ldots,-L
$$

The energies of these electronic orbital angular momentum states do not depend on the sign of $\vec{M}_{\mathrm{L}}$ but only on its absolute number. Because of this usually only the absolute value of $\vec{M}_{\mathrm{L}}$ is given, which is defined as

$$
\Lambda=\left|M_{\mathrm{L}}\right|=0,1,2, \ldots,|L|
$$

Accordingly, every molecules have $L+1$ individual states of which all but $\Lambda=0$ are doubly degenerate. It has to be noted that usually $\vec{L}$ is a bad quantum number. This means its discreet value cannot be determined. Therefore, $\Lambda$ is used instead. Each of these states is assigned a term symbol based on the term symbol nomenclature $(\mathrm{s}, \mathrm{p}, \mathrm{d}, \mathrm{f}, \ldots)$ used for atoms. Starting with $\Lambda=0$ they are labeled $\Sigma, \Pi, \Delta, \Phi$ and so on.

As the atoms retain their electron spin in the molecule the spin also influences the energetic situation in the molecule. Relevant in this depiction is only the total molecular spin $\vec{S}$ which is the sum of all unpaired electron spins of the two atoms. $\vec{S}$ can be both integral or half integral depending on the number of electrons being even or odd. For all molecular states but those with $\Sigma$ term symbol there is a magnetic field along the internuclear axis that is generated by angular motion of the electrons. Since this magnetic field of the electron motion is directed parallel to the internuclear axis the spin vector $\vec{S}$ is, like $\vec{L}$, bound to the internuclear axis. This results in a precession of $\vec{S}$ around the internuclear axis, similar to the precession of $\vec{L}$. Accordingly, $\vec{S}$ is also as ill defined as $\vec{L}$ with only its component along the internuclear axis $h / 2 \pi \vec{M}_{\mathrm{S}}$ as a remaining good quantum number. The left side of Figure 2.5 shows a sketch of the described vectors and their precession. In contrast to the electronic angular momentum the spin is influenced by the direction of the innermolecular magnetic field. Therefore, the energy of the system depends on the sign of $\vec{M}_{\mathrm{S}}$ and in consequence it is equal to $\Sigma$ which can take the following numbers analogous to $\vec{L}$ and $\Lambda$ :

$$
\vec{M}_{\mathrm{S}}=\Sigma=S, S-1, S-2, \ldots,-S .
$$



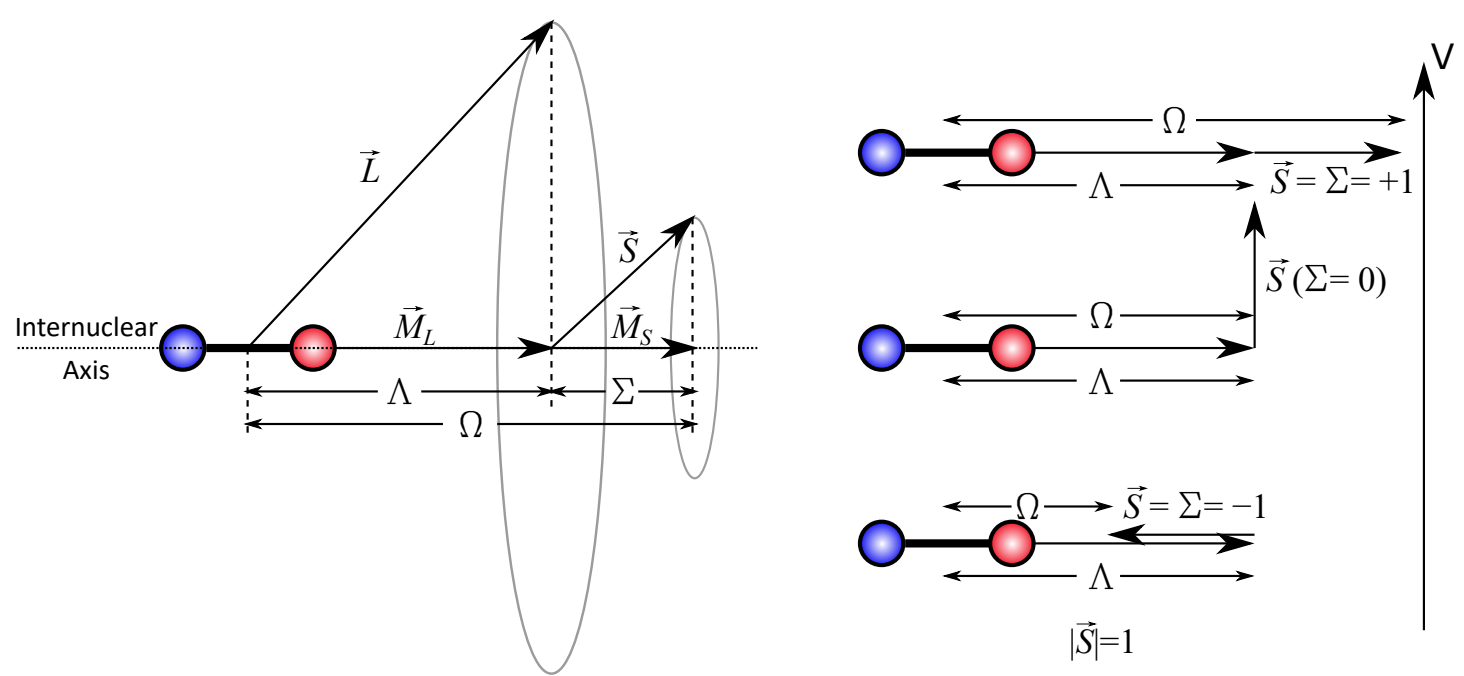

Figure 2.5: The left side of the Figure shows the vector diagram of electronic motion in a generic diatomic molecule. The electronic orbital angular momentum $\vec{L}$ is shown to precess around the internuclear axis. The component along this axis of $\vec{L}$ is labeled as $\vec{M}_{\mathrm{S}}$ with its absolute magnitude $\Lambda$. Adjacent to it is the total molecular spin $\vec{S}$ with its internuclear axis component $\vec{M}_{\mathrm{S}}=\Sigma$. The right side of the figure shows how the sign of $\Sigma$ influences the total electronic angular momentum about the internuclear axis $\Omega$ and the energy of the system. Adapted from Reference [37.

In $\Sigma$ state molecules with $\Lambda=0$ no innermolecular magnetic field is generated from the precession of the electrons. In consequence, $\vec{S}$ is fixed in space and does not precess as long as the molecule is not rotating or influenced by an external magnetic field. Therefore, the quantum number $\Sigma$ can not be defined. It should be made clear that the notation of states with $\Lambda=0$ as $\Sigma$ states has nothing to do with the total spin along the internuclear axis $\Sigma$.

By adding up the quantum numbers for the electronic angular momentum $\Lambda$ and $\Sigma$ the total electronic angular momentum

$$
\Omega=\Lambda+\Sigma
$$

is gained.

Every $\Lambda \neq 0$ state is therefore split into $2 S+1$ states as illustrated by the right side of Figure 2.5. This is called the spin multiplicity of the molecule. For the unperturbed $\Sigma$ state no splitting occurs. The spin multiplicity is added as a left superscript and $\Omega$ is added as a right subscript to the term symbol $\Gamma$ to form an unique description for each energy level of the molecule

$$
{ }^{2 S+1} \Gamma_{\Omega}
$$


which displays all quantum mechanical properties of the molecular state. For example a molecule with $L=2$ and $S=1$ can be found in the states ${ }^{3} \Sigma,{ }^{3} \Pi_{0},{ }^{3} \Pi_{1},{ }^{3} \Pi_{2},{ }^{3} \Delta_{1}$, ${ }^{3} \Delta_{2}$ and ${ }^{3} \Delta_{3}$

The energetic spacing between the different $\Lambda$ states is usually much higher than between the multiplet states with the lowest $\Lambda=0$ quantum number forming the energetically lowest state. $\Lambda=1$ is usually the next higher one and so on. The multiplet states energy is either proportional or anti-proportional to its $\Omega$ value depending on the sign of the spin-orbit constant. States with the lowest $\Omega$ as the energetically lowest state are called regular. They are sometimes indicated by a right subscript $r$ on the term symbol. Irregular states get a right subscript $\mathrm{i}$ and has the lowest $\Omega$ as the energetically highest state.

In a diatomic and also every linear molecule every plane that includes all nuclei is a mirror plane. In non-degenerate states, which for diatomic molecules are compromised by the $\Sigma$ states, the electronic eigenfunction remains either unchanged or changes signs upon reflection on such a plane. This property is relevant for the classification of the electronic state and is therefore added to the term symbol as a right superscript. $\Sigma^{+}$ for unchanging eigenfunctions and $\Sigma^{-}$if they change sign upon reflection.

\subsubsection{Hund's Cases}

The descriptions above about the electronic motion in a diatomic molecule are made under the assumption that the molecules nuclei are fixed in space and not rotating or vibrating. In reality a molecule rotates and vibrates simultaneously to its electron motion and all these are coupled with each other to varying degrees.

The molecular vibration takes the form of several discreet vibrational states in the wells of the electronic potential energy curves. Figure 2.7 from the next section shows an example of the potential energy curves of three electronic states of the NO molecule along the internuclear distance $r$ with some of the vibrational state structure. These curves are cuts of the potential energy surface of the NO molecule. The vibrational motion occurs along this potential energy surface with a certain vibrational energy. The vibrational energy of each electronic state can be approximately described by the vibrational term

$$
G(v)=\tilde{v}_{0}\left(v+\frac{1}{2}\right)-\tilde{v}_{0} \chi_{\mathrm{e}}\left(v+\frac{1}{2}\right)^{2}
$$

which is added to electronic ground state energy. $\tilde{v}_{0}$ is the energetic spacing in wave numbers between two vibrational levels in a harmonic oscillator, $v$ is the vibrational states quantum number and $\chi_{\mathrm{e}}$ the anharmonicity constant to adjust for the anharmonicity of the oscillation. 

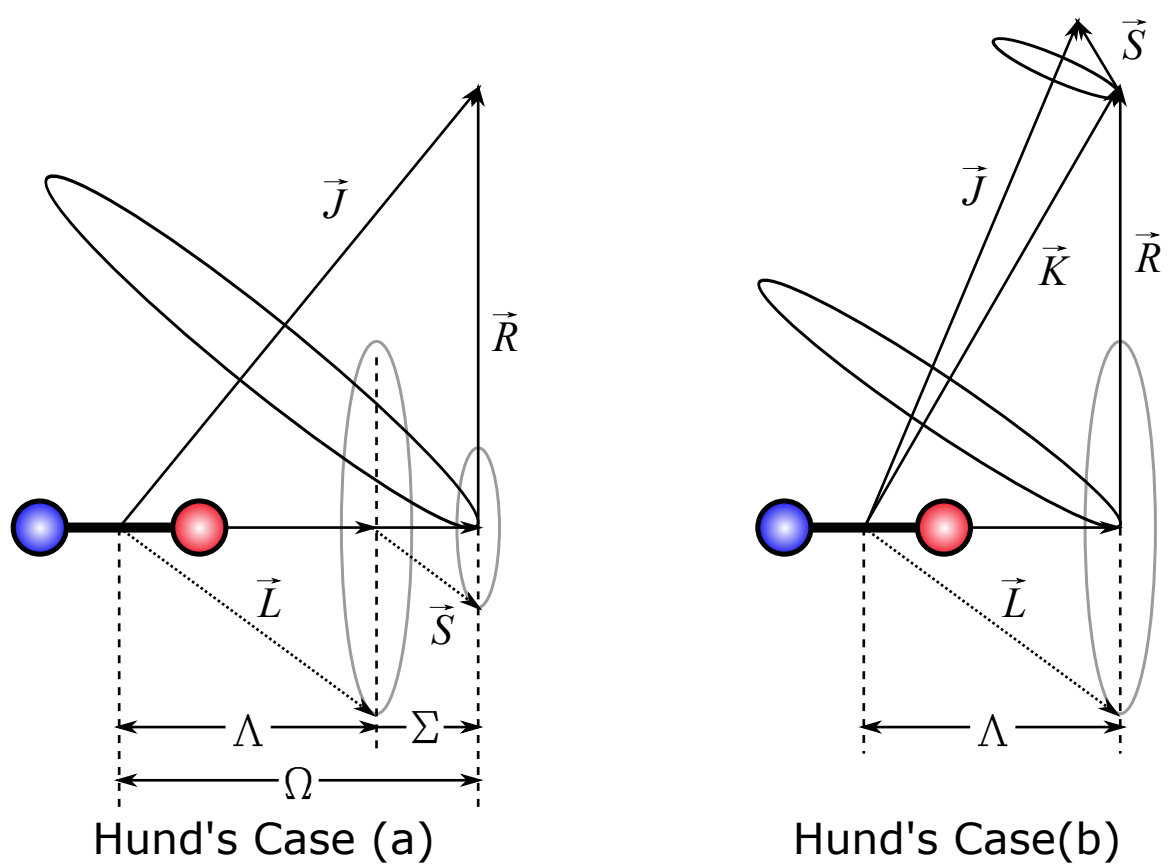

Hund's Case(b)

Figure 2.6: Vector diagram for Hund's case (a) and (b) of a diatomic molecule. In Hund's case (a) the coupling between the nuclear rotation $\vec{R}$ and the electronic motion is negligible while the coupling of $\vec{L}$ and $\vec{S}$ to the internuclear axis is very strong. In consequence its components along the internuclear axis $\Lambda$ and $\Sigma$ are constant and well defined. $\vec{L}$ and $\vec{S}$ combine with the nuclear angular momentum $\vec{R}$ to the total angular momentum vector of the molecule $\vec{J}$ with it internuclear axis component $\Omega$. In Hund's case (b) $\vec{S}$ is no longer coupled or bound to the internuclear axis. This makes $\Sigma$, and in consequence $\Omega$ no longer applicable. $\Lambda$ and $\vec{R}$ combine to the total angular momentum apart from spin vector $\vec{K}$. Adapted from Reference [37.

As each electronic state contains a vibrational structure, each vibrational state likewise contains a rotational structure. The rotational structure can appear to be erratic on first sight because the rotation of a molecule is coupled both to the vibrational and to the electronic motion of the molecule and also strongly influenced by the symmetry of the molecule ${ }^{[38}$. The influence of the coupling between rotation and vibration is taken into by different rotational constants $B_{v}$ for each vibrational state $v$ of the molecule. $B_{v}$ is also different for each direction of the rotation. This is caused by the fact that each direction has a different moment of inertia $I_{\mathrm{i}}$ depending on the molecular structure and follows the equation

$$
B_{v}=\frac{h}{8 \pi^{2} I_{\mathrm{i}}}
$$

For diatomic molecules, both rotational axis perpendicular to the bond have equal moments of inertia. 
The order and spacing of the rotational structure is governed by the interaction between the nuclear angular momentum vector $\vec{R}$ and the electronic motion of both angular momentum $\vec{L}$ and spin $\vec{S}$ and their coupling to the internuclear axis. Hund formulated 1926 a system of five cases called Hund's cases (a), (b), (c), (d) and (e). These cover the possible combinations of couplings between these vectors. Most molecules can be well described by one of these Hund's cases but are actually more accurately described by an intermediate case between these limiting cases. This work will focus on Hund's case (a) and (b) because they work best to describe the properties of NO in its lower electronic states.

The left side of Figure 2.6 shows the vector diagram for Hund's case (a). In this case both the electronic orbital angular momentum $\vec{L}$ and the total electronic spin $\vec{S}$ are strongly coupled to the internuclear axis and very weakly coupled to the nuclear angular momentum $\vec{R}$. This results in a precession around the molecular bond. This means both components along the internuclear axis of $\vec{L}$ and $\vec{S}, \Lambda$ and $\Sigma$, are well defined constants that add up to the total electronic motion along the internuclear axis $\Omega$. $\Omega$ and $\vec{R}$ then add up and form the total angular momentum vector $\vec{J}$. $\vec{J}$ has a constant direction and length and both $\Omega$ and $\vec{R}$, together with the internuclear axis, rotate around it. The energy of the rotation can be best described by a symmetric top with $\Omega$ as the angular momentum around the figure axis. If we neglect centrifugal stretching the rotational energy can be described as

$$
F(J)=B_{v}\left(J(J+1)-\Omega^{2}\right)+A \Omega^{2} .
$$

$B_{v}$ is the rotational constant of the vibrational state $v . A$ is the rotational constant of the electronic component. As $\Omega$ is the internuclear axis component of $J$, states where $J<\Omega$ do not occur and it can only take the values

$$
J=\Omega, \Omega+1, \Omega+2, \ldots
$$

This also means that since $R$ is always integral both $J$ and $\Omega$ are always both integral or half-integral depending on whether the total number of electrons is even or odd.

In Hund's case (b), illustrated by the right side of Figure 2.6, the electronic orbital angular momentum $\vec{L}$ is still strongly coupled to the internuclear axis but the spin $\vec{S}$ is not. This occurs mainly when no inner-molecular magnetic field is generated by electronic motion because $\Lambda=0$. In consequence the spin is not coupled to the innernuclear axis. This effect can also occur in small molecules when the intensity of electronic motion is sufficiently small. In consequence $\Lambda$ is still well defined but $\Sigma$ is not and so is $\Omega$. Therefore $\vec{J}$ cannot be constructed from the nuclear rotation $\vec{R}$ and $\Omega$. 
Instead the total angular momentum vector without spin $\vec{K}$ is generated by adding $\vec{R}$ to $\Lambda$. Analogous to the $\vec{J}$ in Hund's case (a) above it can only take the values

$$
K=\Lambda, \Lambda+1, \Lambda+2, \ldots
$$

starting with $\Lambda=0$ where $K=R$. The spin $\vec{S}$ and angular momentum $\vec{K}$ add up to the total angular momentum $\vec{J}$ which can take the values

$$
J=|K+S|,|K+S-1|,|K+S-2|, \ldots,|K-S| .
$$

Accordingly $J$ and $S$ are always both integral or half-integral depending on whether the number of electrons is even or odd. Since the splitting of the $K$ quantum number is much larger than the splitting of the $J$ quantum number we get a series of $K$ states consisting of $2 S+1$ states with different $J$ values ranging from $|K+S|$ to $|K-S|$.

\subsubsection{Quantum States of Nitric Oxide}

In the context of this work the lowest excited electronic state of $\mathrm{NO}^{2} \Sigma^{+}$is used for the preparation of highly vibrationally excited NO via stimulated emission pumping (SEP). Furthermore, NO in its ground electronic state $\mathrm{X}^{2} \Pi_{r}$ can be detected through resonance enhanced multi-photon ionization (REMPI) along allowed transitions of the $\gamma$-band system 40 . The ionization occurs via the $\mathrm{NOA}^{2} \Sigma^{+}$state to the $\mathrm{X}^{1} \Sigma^{+}$state of the nitrosyl ion $\mathrm{NO}^{+}$. Individual transitions are in the following labeled as:

$$
\gamma\left(v^{\prime \prime}, v^{\prime}\right)
$$

$v^{\prime}$ is the vibrational state of the ground electronic state $\mathrm{X}^{2} \Pi_{r}$ and $v^{\prime \prime}$ is the vibrational state of the excited electronic state $\mathrm{A}^{2} \Sigma^{+}$. Figure 2.7 shows the calculated energies of these three electronic states and the underlying structure of vibration states. The REMPI scheme used in this work is well understood and is described in detail in an article by Jacobs $\underline{40}$. Previous works have used it with success in various NO scattering experiments 4143 . For the interpretation and selective preparation of certain NO states it is essential to be able to calculate the energy of these states and their transitions with sufficient precision. The following section is based on the remarks found in the thesis of Bastian Krüger 41 which itself is based on the articles by Amiot 44 46. The calculation of diatomic molecules can be done by employing an effective Hamiltonian established by Brown ${ }^{47}$ :

$$
H=H_{0}+H_{\mathrm{SO}}+H_{\mathrm{Rot}}+H_{\mathrm{SR}} .
$$

$H_{0}$ is the Hamiltonian of the molecular vibration, $H_{\mathrm{SO}}$ is the Hamiltonian of the spinorbit interaction, $H_{\mathrm{Rot}}$ is the Hamiltonian of the molecular rotation and $H_{\mathrm{SR}}$ the Hamiltonian of the spin-rotation interaction. 


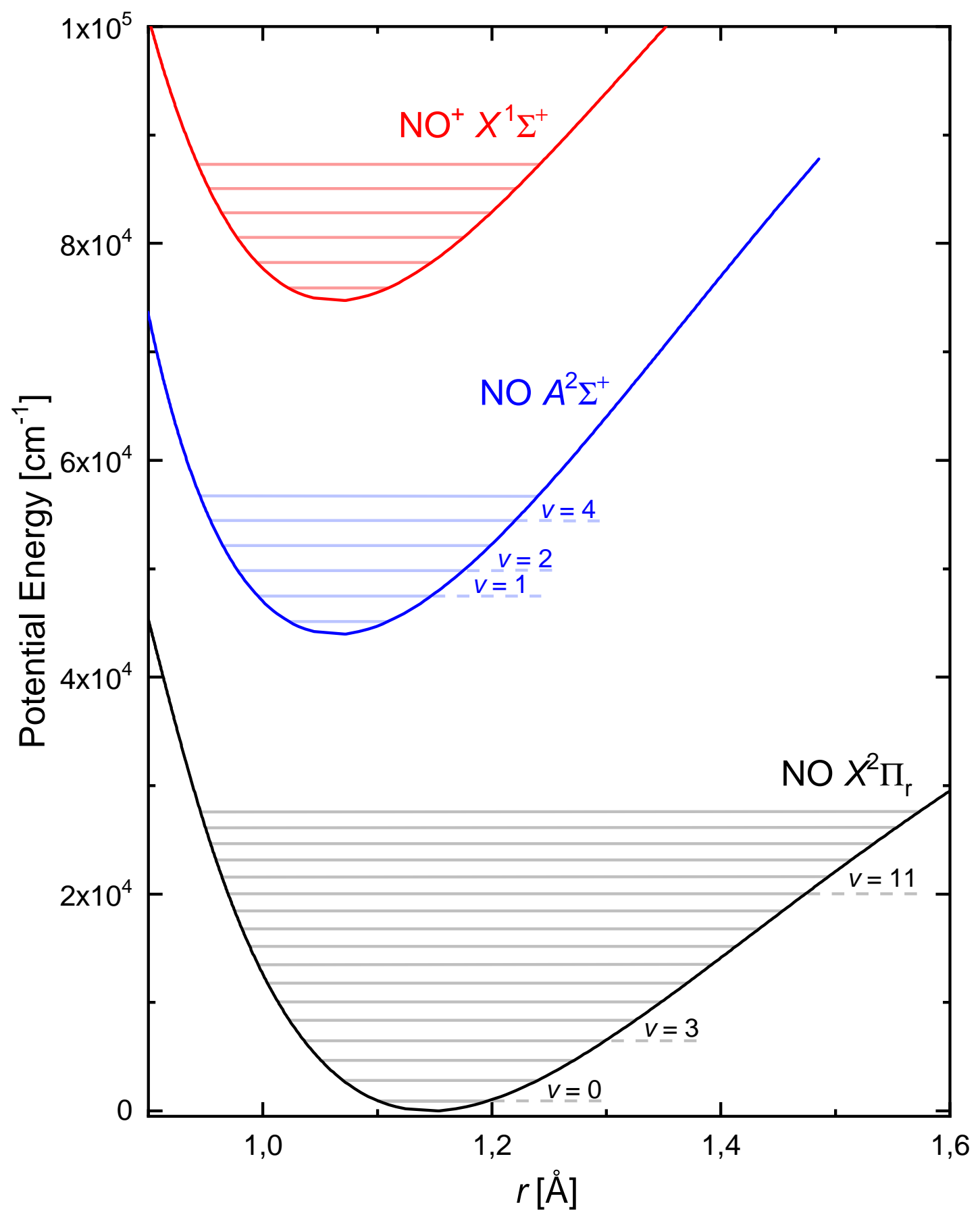

Figure 2.7: Calculated potential energy curves of the electronic states $\mathrm{X}^{2} \Pi_{r}$ and $\mathrm{A}^{2} \Sigma^{+}$of $\mathrm{NO}$ and $\mathrm{X}^{1} \Sigma^{+}$of $\mathrm{NO}^{+}$in vacuum. The lowest vibrational states are added to the potential energy curves in fade colors with states relevant for this work marked with their quantum number $v$. Vibrational state energies are calculated with diatomic constants from Reference 39 . 
Ground State $\mathrm{X}^{2} \Pi_{r}$ NO is best described by the Hund's case (a) according to the Reference [48]. The wave function can therefore be written as

$$
|J M \Omega \epsilon\rangle=\frac{1}{\sqrt{2}}(|J M+\Omega\rangle+\epsilon|J M-\Omega\rangle)
$$

with the quantum numbers of the total angular momentum of the molecule $J$, its component on the lab frame axis $M$ and its component on the internuclear axis $\Omega$. $\epsilon$ is the variable of the molecule's parity and can be either +1 or -1 written as e or $f$. It is translated into the total parity $(+/-)$ by

$$
(-1)^{J-\frac{\epsilon}{2}}
$$

Furthermore, the NO ground state has a spin multiplicity of $2 S+1=2$ with $\Omega$ as either $\Omega=1 / 2$ or $\Omega=3 / 2$. Considering this the Hamiltonian is written as a $2 \times 2$ matrix:

$$
H_{\epsilon}(v, J)=\left(\begin{array}{cc}
H_{11, \epsilon, \Omega=3 / 2}(v, J) & H_{12, \epsilon}(v, J) \\
H_{21, \epsilon}(v, J) & H_{22, \epsilon, \Omega=1 / 2}(v, J)
\end{array}\right)
$$

with the first diagonal element

$$
\begin{aligned}
H_{11, \epsilon, \Omega=3 / 2}(v, J)= & h c\left(T_{\mathrm{X}}+W_{\mathrm{X}}+0.5 A_{\mathrm{X}}+0.5 A_{\mathrm{D}, \mathrm{X}} \cdot z\right. \\
& \left.+\tilde{B}_{\mathrm{X}, v} \cdot z-\tilde{D}_{\mathrm{X}, v} \cdot z(z+1)+\tilde{H}_{\mathrm{X}, v} \cdot z(z+1)(z+2)\right),
\end{aligned}
$$

the second diagonal element

$$
\begin{aligned}
H_{22, \epsilon, \Omega=1 / 2}(v, J)= & h c\left(T_{\mathrm{X}}+W_{\mathrm{X}}-0.5 A \mathrm{X}-0.5 A_{\mathrm{D}, \mathrm{X}} \cdot(z+2)\right. \\
& +\tilde{B}_{\mathrm{X}, v} \cdot(z+2)-\tilde{D}_{\mathrm{X}, v} \cdot z(z+1)(z+4) \\
& +\tilde{H}_{\mathrm{X}, v} \cdot(z+1)\left(z^{2}+8 z+8\right) \\
& \left.-0.5 \epsilon \cdot p_{\Lambda} \cdot(J+0.5)-\epsilon \cdot q_{\Lambda} \cdot(J+0.5)\right)
\end{aligned}
$$

and the non-diagonal elements

$$
\begin{aligned}
H_{12, \epsilon}(v, J)=H_{21, \epsilon}(v, J)= & h c(-\tilde{B} \sqrt{z}+2 \tilde{D} \sqrt{z} \cdot(z+1)-\tilde{H} \sqrt{z} \cdot(z+1)(3 z+4) \\
& \left.+0.5 \epsilon \cdot q_{\Lambda} \cdot \sqrt{z} \cdot(J+0.5)\right)
\end{aligned}
$$

$z$ is used to abbreviate the expression $(J-0.5)(J+0.5)$. $T_{\mathrm{X}}$ denotes the electronic energy of the ground state and $W_{\mathrm{X}}$ the vibrational energy. $\tilde{B}_{\mathrm{X}, v}$ is the rotation constant of the ground state. $\tilde{D}_{\mathrm{X}, v}$ and $\tilde{H}_{\mathrm{X}, v}$ are centrifugal distortion constants of the ground state. $A \mathrm{X}$ and $A_{\mathrm{D}, \mathrm{X}}$ are the constants of spin-orbit splitting. $p_{\Lambda}$ and $q_{\Lambda}$ are constants to describe the $\Lambda$ doubling caused by perturbations with the ${ }^{2} \Sigma^{-}$states. All constants 
beside $T_{0}$ depend on the vibrational state $v$ and are extracted from Reference [46] except for the $\Lambda$ doubling parameters that were taken from Reference [49].

The First Electronically Excited State $\mathrm{A}^{2} \Sigma^{+} \mathrm{NO}$ can be, as a $\Sigma$ state, be best described by Hund's case (b). But in order to simplify the calculations of the transitions $J$ is used instead of the quantum number of nuclear rotation $R$ which would otherwise be the more appropriate choice. The energies for the e and $f$ states are described as

$$
H_{\epsilon}=h c\left(T_{\mathrm{A}}+W_{\mathrm{A}}+\tilde{B}_{\mathrm{A}, v} \cdot z-\tilde{D}_{\mathrm{A}, v} \cdot z^{2}+0.5 \epsilon \cdot \gamma_{\mathrm{A}}(J+0.5-\epsilon)\right) .
$$

$T_{\mathrm{A}}$ is the electronic energy of the $\mathrm{A}^{2} \Sigma^{+}$state and $W_{\mathrm{A}}$ the vibrational energy of the same. $B_{\mathrm{A}}$ is the rotational constant and $D_{\mathrm{A}}$ is the constant of centrifugal distortion. $\gamma_{\mathrm{A}}$ is the constant of the spin-rotation interaction. All the constants are found in Reference [49]. The selection rules for electronic transitions such as $\left(\mathrm{A}^{2} \Sigma^{+}(v, R) \leftarrow \mathrm{X}^{2} \Pi_{r}(v, J)\right)$ are given by Hollas' book 38 as

$$
\begin{gathered}
\Delta J=0, \pm 1 \\
\Delta S=0 \\
\Delta \Lambda=0, \pm 1 \\
+\leftrightarrow-,+\leftrightarrow+,-\leftrightarrow-
\end{gathered}
$$

The resulting branches are shown in Figure 2.8. The individual transitions are labeled as $\mathrm{P}, \mathrm{Q}$ or $\mathrm{R}$ depending on whether $\Delta J$ is $-1,0$ or +1 and a subscript describing the spin label (F1 or F2) of the initial state as the second digit and of the destination state as the first digit. The spin label for Hund's case (a) is assigned regarding the $\Sigma$ quantum number. The state gets the spin label F1 if $\Sigma=1 / 2$ and F2 if $\Sigma=-1 / 2$. For Hund's case (b) the spin label is assigned differently because $\Sigma$ is undefined. Here it depends on the direction of the total spin vector $\vec{S}$ in relation to the total angular momentum of the molecule without spin $\vec{R}$. So for every two rotational states of the $\mathrm{A}^{2} \Sigma^{+}$state with the same $R$ the state with the larger $J$ has the spin label F1 and the one with the lower $J$ has the spin label F2. Examples of this can be seen in Figure 2.8. The method used to extract relative population distribution of $\mathrm{NO}$ quantum states from a 1+1 REMPI spectrum is described in Reference [40]. 


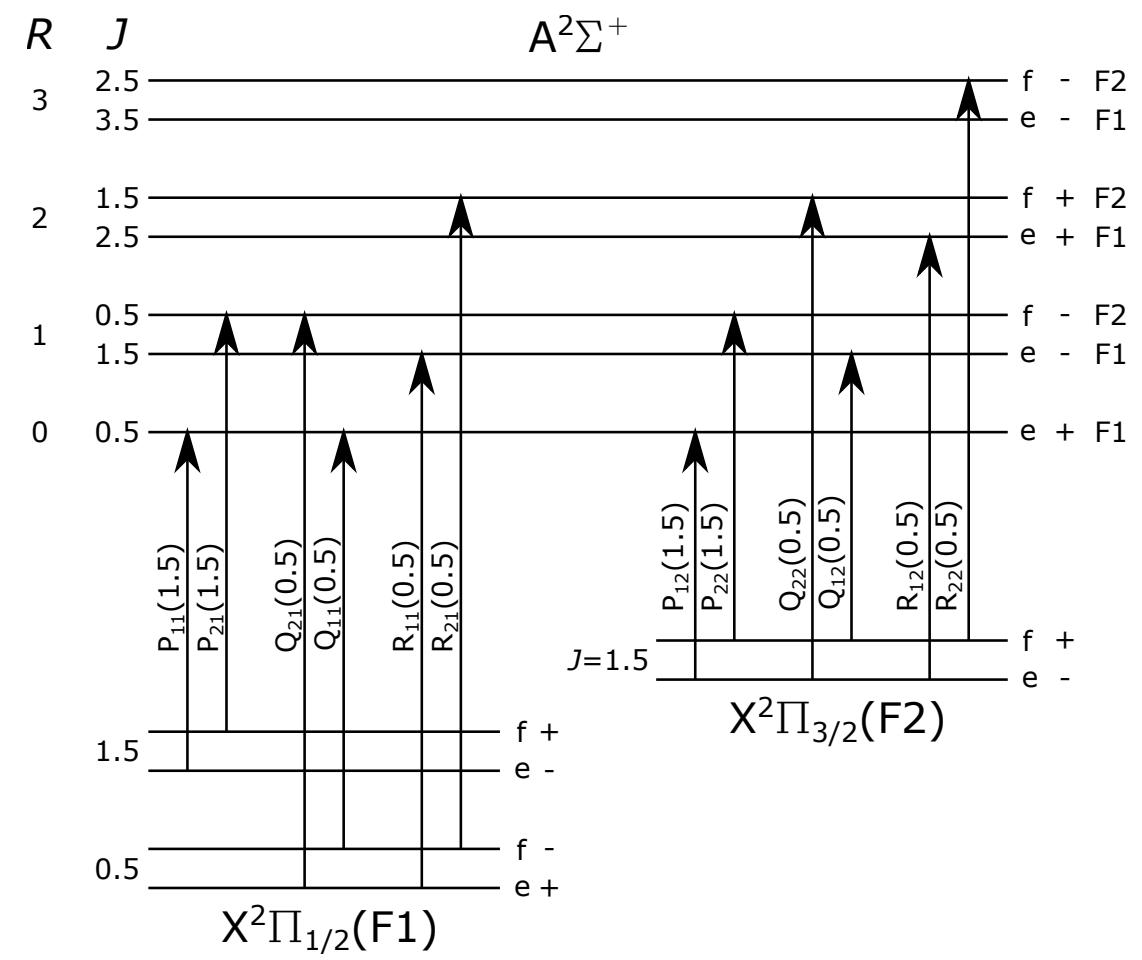

Figure 2.8: Rotational branches of the allowed transitions for a generic vibrational band of the $\gamma$-band system. Adapted from Reference [48.

\subsection{Surface Scattering with Nitric Oxide}

As shown in Figure 2.9 molecules can interact through different mechanisms referred to as channels when they collide with a solid surface. They can stick to the surface by getting trapped in the chemi- or physisorption well. Depending on the depth of the well and the surface temperature this state can be stable. The alternative is that they bounce back directly when they hit the repulsive potential of the surface. Both cases are shown schematically in Figure 2.10. In the first case, the molecule is trapped long enough to equilibrate with the surface temperature. This occurs through coupling of the molecular vibrations to phonons. These interactions take about $\tau_{p h}>1 \mathrm{ps}$ to occur and therefore require the molecule to dwell for some time on the surface. When the molecule desorbs from the surface its translational energy and vibrational and rotational population adjust to the surface temperature. In the second case, the interaction time between surface and molecule is not long enough for equilibration to occur. On this small time scale of $<1$ ps only interactions between the molecular vibration and the surface electrons with $\tau_{e l} \approx 0.1-1 \mathrm{ps}$ for d-band metals can occur 51 . This relaxation channel is not anticipated by the Born-Oppenheimer approximation which is usually 


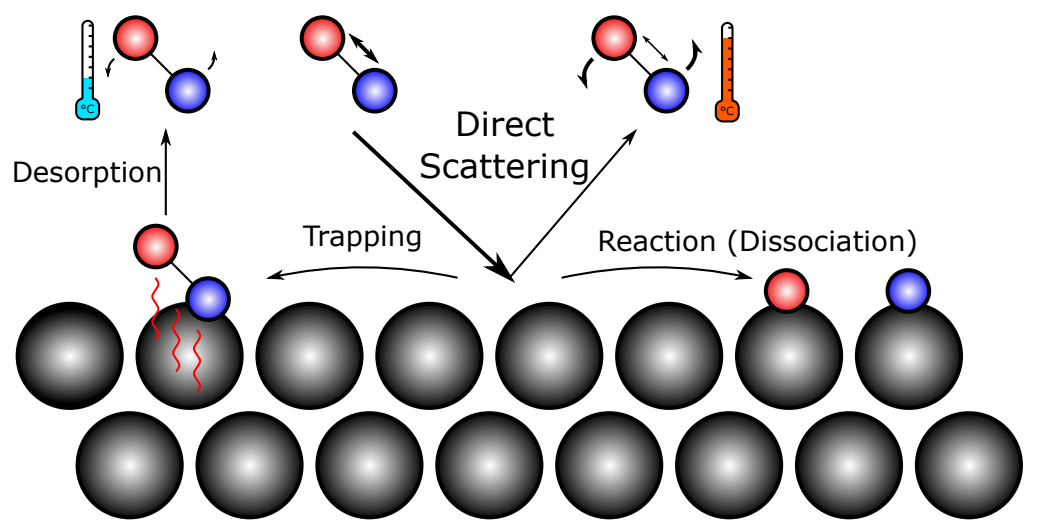

Figure 2.9: Schematic sketch of a selection of possible gas-surface scattering channels that can potentially occur during the scattering of a fast molecule $(1 \mathrm{eV})$ from a room temperature surface $(300 \mathrm{~K})$. This work is mostly concerned with direct scattering events. Trapping desorption and and surface reactions occur only as side effects and expenses were incurred to suppress them in experiment. The arrows on the scattered molecules represent vibrational and rotational excitation whose intensity is shown by the arrows thickness. Thermometer icons indicate the increase in rotational temperature of the molecule after direct scattering. It is generated through the conversion of translational to rotational excitation. During trapping desorption the rotational temperature equilibrates with the surface resulting in a lower rotational temperature.

used to describe molecular potentials and reactions so that for a sufficient theoretical description this approximation cannot be used.

Of course there are several intermediate channels such as multi-bounce collision where the molecule is not trapped but jumps over two or three binding sides before it desorbs again. Another channel can be reactions on the surface such as dissociation of the molecule or reactions with other adsorbed particles.

\subsubsection{Born-Oppenheimer Approximation and Breakdown}

The Born-Oppenheimer approximation is an important theoretical simplification used to make the description of the potentials found in molecules feasible. Nevertheless, it is based on assumptions which are not necessarily always true. So it can occur that theoretical predictions turn out to be off because they rely on the Born-Oppenheimer approximation. This leads to the often cited Born-Oppenheimer breakdown which is still often hard to accommodate for if one wants to describe molecular interaction theoretically. 


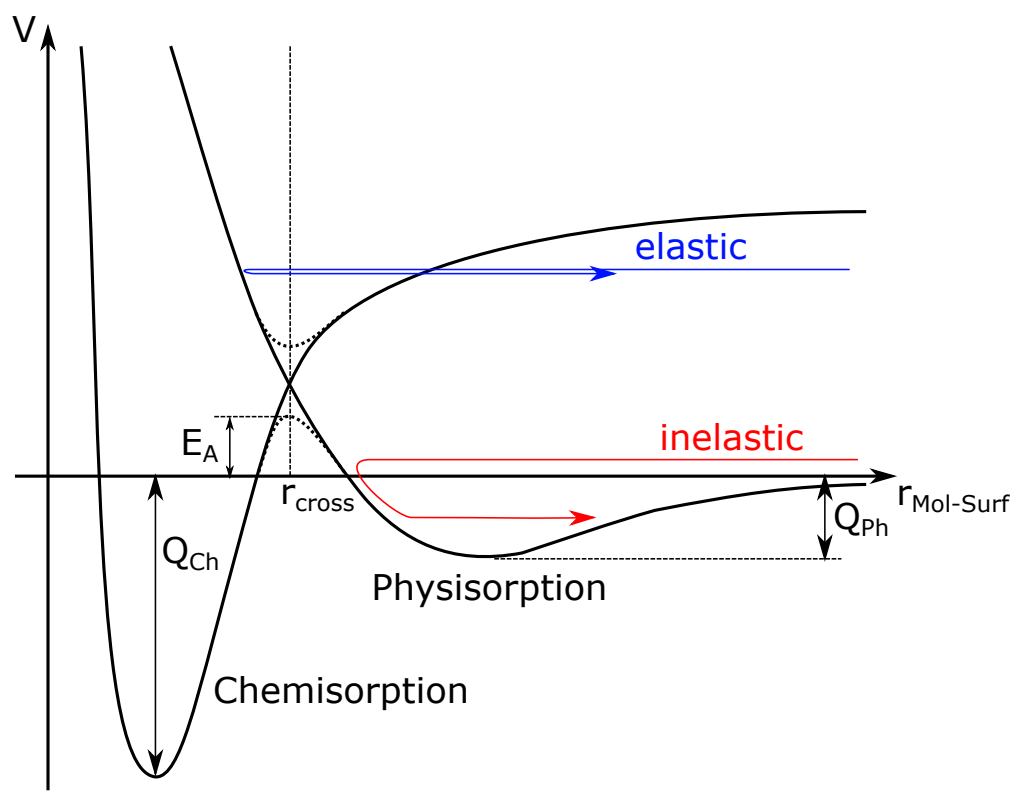

Figure 2.10: Schematic potential energy $(V)$ curves of the physisorption well with the depth $Q_{\mathrm{Ph}}$ and chemisorption well with the depth $Q_{\mathrm{Ch}}$. The curves cross at $r_{\text {cross }}$ and form an activation barrier with the height $E_{\mathrm{A}}$. Physisorption by energy loss an subsequent trapping in the potential well is shown by the red arrow. Direct scattering of a fast molecule (with higher energetic potential) is shown by the blue arrow. The arrow indicates the molecules direction. Figure taken and modified from Reference [50].

The explanations in this sections are based on the articles by John Tully [52, 53. The structure of an atom can be described by the time-independent Schrödinger equation postulated in 1926 [54].

$$
\hat{H}|\Psi\rangle=E|\Psi\rangle
$$

$\hat{H}$ is the Hamiltonian operator. $\Psi$ is the wave function of the observed system that can be both an atom or a group of atoms like a molecule with the particle's energy eigenvalue $E$. It has to be noted that this equation is only analytically solvable for particles with two or less bodies like the hydrogen atom with its single proton nucleus and the single orbiting electron. Another example for a solvable system is the helium cation if you regard its nucleus as a single body although it contains two protons and two neutrons. This assumption is valid because the internuclear interactions are usually not coupled to the electrons. One reason for this is the large difference in potential energy of the nuclei and electrons. The other reason is the difference in size between the nucleus and the electron orbitals. So for electronic dynamics the nuclei can be considered as single point charges. Any system with more independent bodies requires a numerical approach. This works by calculating the potential energy for each combination of 
relative positions of all the relevant bodies. As each body's position has to be varied along the three space dimensions the time-independent wave function $\Psi$ requires $3 N$ dimensions to describe the total particle properly with $N$ being the number of bodies. Depending on the resolution of such a potential energy surface mapping the computing time can easily exceed any reasonable amount for even relatively simple molecules. Therefore, it is absolutely necessary to simplify the equation with approximations to cut down the computing time to a manageable magnitude. One such approximation was published already in the year after Schrödinger published his equation in 1927 by Born and Oppenheimer [55. In simple terms it assumes that electrons move much faster than nuclei. A proton is 1836 times heavier than an electron. As the moment of inertia scales with mass, the nucleus' moment of inertia is significantly higher than the electrons moment of inertia. From the perspective of the electron a nucleus is essentially a fixed point in space and any movement of the nucleus is met with an immediate response from the nearby electrons, while the nucleus barely reacts to the electron movement. In consequence, this means that the nuclear and electron motion are decoupled from each other and can be described individually.

One example for the application of the Born-Oppenheimer approximation is the hydrogen cation $\mathrm{H}_{2}^{+}$which consists of two protons and an electron. Under normal circumstances its Schrödinger equation would not be analytically solvable but under the Born-Oppenheimer approximation the two protons are fixed in space and act as a single particle from the electron's perspective. This reduces the system to two bodies. Keep in mind that in contrast to the $\mathrm{He}^{+}$cation the protons in $\mathrm{H}_{2}^{+}$are not compressed into the nucleus but separated by a molecular bond of about $1 \AA$ length, which is about the size of the electron orbital.

For the formal description of the Born-Oppenheimer approximation one has to start with the time-independent Schrödingers equation for a generic molecule

$$
\hat{H}|\Psi(\vec{r}, \vec{R})\rangle=E(r, R)|\Psi(\vec{r}, \vec{R})\rangle .
$$

The molecular wave function $\Psi(\vec{r}, \vec{R})$ is dependent on the electron positions $\vec{r}$ and the nuclear positions $\vec{R}$. The ensemble of infinite eigenvalues spans a potential energy surface $E(r, R)$ with the electron coordinates $\vec{r}$ and nuclei coordinates $\vec{R}$. If spin-orbit coupling is neglected the full non-relativistic molecular Hamiltonian operator $\hat{H}$ can be written as

$$
\hat{H}=\hat{T}_{\alpha}+\hat{T}_{\mathrm{e}}+V(\vec{r}, \vec{R}) .
$$

The first addend describes the kinetic energy operator

$$
\hat{T}_{\alpha}=-\frac{\hbar^{2}}{2} \sum_{\alpha} \frac{\nabla_{\mathrm{R}}^{2}}{M_{\alpha}}
$$


of the nucleus $\alpha$ with the mass $M_{\alpha}$. The second addend stands for the kinetic energy operator of the electron

$$
\hat{T}_{\mathrm{e}}=-\frac{\hbar^{2}}{2} \sum_{i} \frac{\nabla_{\mathrm{r}}^{2}}{m_{\mathrm{e}}}
$$

with the electron mass $m_{\mathrm{e}}$. The last addend $V(\vec{r}, \vec{R})$ includes all potential energy operators for the inter-particle interactions. The last two addends can be summarized to the Hamiltonian $H_{\mathrm{el}}(\vec{r} ; \vec{R})$ of the whole system except $T_{\alpha}(\vec{R})$ :

$$
\hat{H}=\hat{T}_{\alpha}(\vec{R})+\hat{H}_{\mathrm{el}}(\vec{r} ; \vec{R}) .
$$

Note that the coordinates $\vec{r}$ and $\vec{R}$ of $\hat{H}_{\mathrm{el}}(\vec{r} ; \vec{R})$ are now separated by a semicolon to emphasize that this particular Hamiltonian depends only parametrically on $\vec{R}$. Like the Hamiltonian the molecular wave function $\Psi(\vec{r}, \vec{R})$ is, in accordance with the BornOppenheimer approximation, also separated in wave functions of nuclear motion $\Omega_{i}(\vec{R})$ and the adiabatic electronic wave functions $\Phi_{i}(\vec{r} ; \vec{R})$ of the electronic state $i$

$$
\Psi(\vec{r}, \vec{R})=\sum_{i} \Phi_{i}(\vec{r} ; \vec{R}) \Omega_{i}(\vec{R})
$$

$\Phi_{i}(\vec{r} ; \vec{R})$ is a set of infinite wave function for each nucleus position $\vec{R}$ so that when the electronic Hamiltonian $\hat{H}_{\mathrm{el}}(\vec{r} ; \vec{R})$ from equation 2.30 is applied to them we get a set of infinite eigenvalues which span the state $i$ specific potential energy surfaces $E_{i}(\vec{R})$ in dependence of the nuclei positions $R$ :

$$
\hat{H}_{\mathrm{el}}\left|\Phi_{i}(\vec{r} ; \vec{R})\right\rangle=E_{i}(r, R)\left|\Phi_{i}(\vec{r} ; \vec{R})\right\rangle .
$$

The ground electronic state is labeled with $i=0$ and the excited states with $i>0$. With these energy surfaces one can simulate possible reaction pathways if the initial conditions are known.

The formal justification of the Born-Oppenheimer approximation becomes visible when equation 2.31 is substituted by the Hamiltonian of equation 2.27 and multiplied from the left by $\Phi_{i}^{*}(\vec{r} ; \vec{R})$ and the result then integrated over $\vec{r}$ :

$$
\sum_{j}(\underbrace{\sum_{\alpha} \frac{-\hbar^{2}}{2 M_{\alpha}} \nabla_{\mathrm{R}}^{2}}_{T_{\alpha}}+E_{i}(\vec{R})-E_{i j}-\underbrace{\sum_{i}^{\frac{-\hbar^{2}}{2} D_{j i}(\vec{R})}}_{T_{i j}^{\prime \prime}}-\underbrace{\left.\sum_{i \neq j} \frac{\hbar^{2}}{2} d_{j i}(\vec{R}) \nabla_{\mathrm{R}}\right)}_{T_{i j}^{\prime}}) \Omega_{j}(\vec{R})=0
$$

with the first derivative matrix elements

$$
d_{i j}(\vec{R})=-\sum_{\alpha} \frac{\int \Phi_{i}^{*}(\vec{r} ; \vec{R})\left[\nabla_{R} \Phi_{j}(\vec{r} ; \vec{R})\right] \mathrm{d} \vec{r}}{M_{\alpha}}
$$




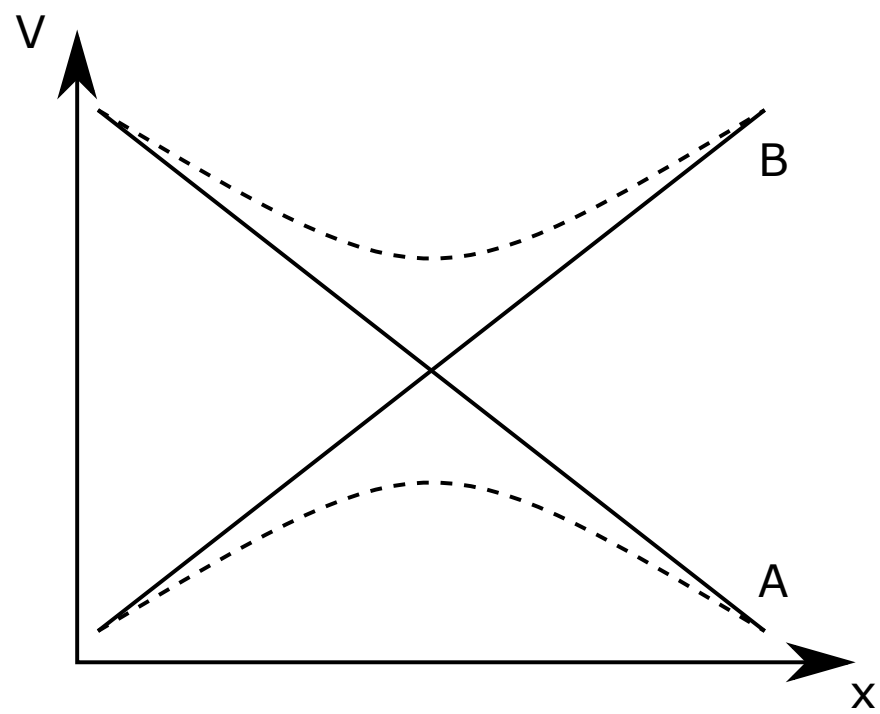

Figure 2.11: Crossing of two potential energy curves for generic particles A and B along the $x$-axis. The dashed line depicts the adiabatic picture under the Born-Oppenheimer approximation where the electronic structure adapts instantly to the changing potential energy during the crossing. In this picture A phases to B radiationlessly but cannot transit from the lower curve to the upper one without external excitation. The solid line depicts the nonadiabatic picture where electrons cannot adept during the crossing. This occurs if the the transition along $x$ occurs too fast for the electrons to react leading to the breakdown of the Born-Oppenheimer approximation. In this case A cannot transit to B.

and second derivative matrix elements

$$
D_{i j}(\vec{R})=-\sum_{\alpha} \frac{\int \Phi_{i}^{*}(\vec{r} ; \vec{R})\left[\nabla_{R}^{2} \Phi_{j}(\vec{r} ; \vec{R})\right] \mathrm{d} \vec{r}}{M_{\alpha}}
$$

$T_{\alpha}$ is the representation of the nuclear motion from Equation $2.30 E_{i}(\vec{R})$ is the potential energy surface from Equation 2.32 The off-diagonal elements $T_{i j}^{\prime}$ and $T_{i j}^{\prime \prime}$ and $E_{i j}$ describe the interaction between different electronic states $i$ and $j$ when $i \neq j$. Under the assumption of adiabaticity $E_{i j}$ is zero while $T_{i j}^{\prime}$ and $T_{i j}^{\prime \prime}$ are non-zero but still negligibly small. $T_{i j}^{\prime}$ and $T_{i j}^{\prime \prime}$ are governed by the parameters $d_{i j}(\vec{R})$ and $D_{i j}(\vec{R})$ whose squares are according to Tully ${ }^{[53}$ comparable with typical rotational or vibrational energies. Tully assumes values of $10^{-4}$ for the rotation and $10^{-2}$ for vibration while the square of $d_{i j}(\vec{R})$ and $D_{i j}(\vec{R})$ get intensities of $10^{-6}$ and $10^{-8}$, respectively, making them several orders of magnitude smaller in comparison. The diagonal element $T_{i i}^{\prime \prime}$ with $D_{i i}(\vec{R})$ in the case of $i=j$ is often used as a correction term for $E_{i}(\vec{R})$ but in this case it is also neglected for the sake of simplicity and because its intensity is only around the energy of rotation with a numerical value of $10^{-4}$.

This approximation holds true if we observe isolated adiabatic potential energy curves 
or surfaces where no coupling to other states can occur. This is the case when $d_{i j}(\vec{R})$ and $D_{i j}(\vec{R})$ are weak but this is not always given. In some cases the energy gap between two electronic states can become so small that the coupling between them becomes relevant. An example for this is when two diabatic energy curves cross as depicted in Figure 2.10 at $r_{\text {cross }}$. Here, we have the physisorption curve where the electronic structures do not interact with the surface and the chemisorption curve where the electrons of the particle are bound to the electron structure of the surface. The solid lines depict adiabates that illustrate the picture where an incoming non-bound particle will travel along the physisorption curve. In the end it will be reflected from the repelling potential (red arrow) although at $r_{\text {Mol-Surf }}<r_{\text {cross }}$ the chemisorption would be energetically preferable. In the adiabatic picture a so-called avoided crossing occurs at $r_{\text {cross }}$ forming a energy barrier which can be traversed resulting in a radiationless transition from the the unbound to the bound particle. Figure 2.11 shows a more general picture of such an avoided crossing between the diabatic potential curves A and B.

It should be made clear that since the ratio between the nucleus and electron mass $M_{\mathrm{N}} / m_{\mathrm{e}}$ is neither infinite nor zero both the adiabatic and diabatic picture are in any situation at to some extend partially valid and at the crossing region both pathways can be taken depending on the present parameters. To determine the tendency of these so-called non-adiabatic transitions in the adiabatic picture the Massey parameter can be used ${ }^{56}$.

$$
\xi=\left|\frac{\hbar v_{\mathrm{N}} d_{i j} M_{\alpha}}{E_{i}(R)-E_{j}(R)}\right|
$$

$v_{\mathrm{N}}$ is the classical velocity of nuclear motion. $d_{i j}$ is the coupling matrix element from Equation 2.34 and $E_{i}\left(r_{\text {cross }}\right)-E_{j}\left(r_{\text {cross }}\right)$ is the energetic distance between the two observed electronic states. With higher $\xi$ the coupling between these states becomes more intense and the non-adiabatic transition becomes more probable. In the case of $\xi \gg 1$ the system can be almost solely described by the diabatic picture and in the case of $\xi \ll 1$ the contrary is true. As stated above $d_{i j}$ is a rather small parameter so that $E_{i}\left(r_{\text {cross }}\right)-E_{j}\left(r_{\text {cross }}\right)$ needs to be equally small. In consequence, the adiabatic picture is usually sufficient to describe most situations. 


\subsubsection{Scattering of vibrationally excited NO}

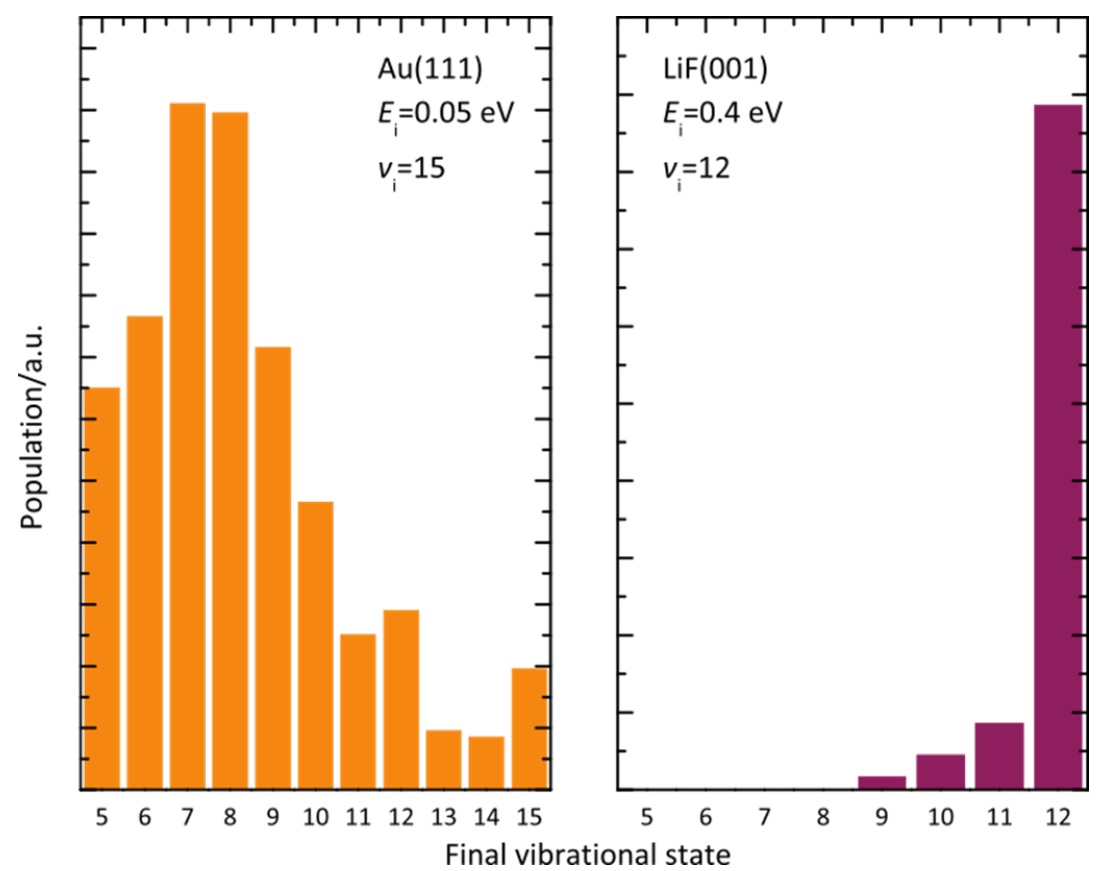

Figure 2.12: Vibrational distribution of NO scattering experiment from $\mathrm{Au}(111)$ and $\mathrm{LiF}(001)$ of Huang et al. from 2000. It shows that vibrational relaxation occurs more during the collision with gold than with LiF. Taken and altered from Reference 9 .

Previous works by Huang ${ }^{[9}$, Bartels $\underline{43}$, Golibrzuch $\underline{42}$, Steinsiek $\frac{59}{}$, Krüger $\underline{41}$ and Wagner ${ }^{58}$ have investigated the vibrational excitation and relaxation of small molecules such as $\mathrm{NO}$ or $\mathrm{CO}$ when directly scattered from different metallic and insulator surfaces. The general picture, as shown in Figure 2.12, is that vibrational deexcitation occurs more efficiently on metals where the molecular vibration can couple to the energetically close electronic states of the surface. For insulators such as LiF this mechanism is not possible. Because of the band gap there is no electron density with matching energy available.

Later experiments with $\mathrm{NO}$ scattering from $\mathrm{Au}(111)$ and $\mathrm{Ag}(111)$ also showed that the relaxation is not only dependent on the translational energy of the incident molecule. It is also dependent on the electron affinity of the molecule and the work function of the surface. From Figure 2.13 it is apparent that if the work function $\Phi$ and the molecular energy at the outer turning point of the molecule $E_{\mathrm{v}}\left(v, r_{\text {outer }}\right)$ are sufficiently close $\left(\left|\Phi-E_{\mathrm{v}}\left(v, r_{\text {outer }}\right)\right|<3 \mathrm{eV}\right)$ almost all molecules relax upon collision with the surface from their initial to a lower vibrational state. Also beyond this point the final vibrational states shift to lower states the smaller $\left|\Phi-E_{\mathrm{v}}\left(v, r_{\text {outer }}\right)\right|$ is. This can be seen in 


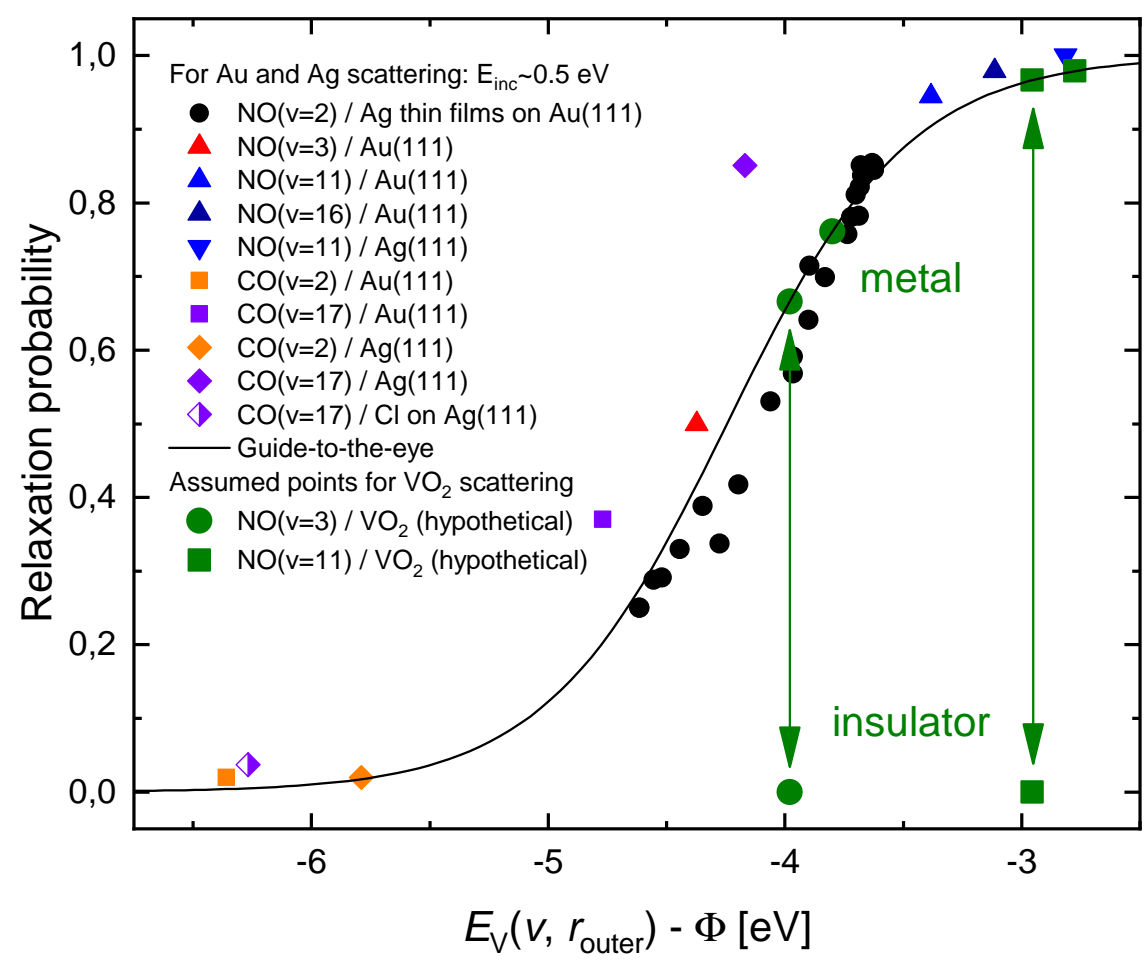

Figure 2.13: Relaxation probability during a direct scattering event plotted against the difference between vertical electron binding energy $E_{\mathrm{v}}\left(v, r_{\text {outer }}\right)$ of $\mathrm{NO}$ or $\mathrm{CO}$ and work function $\Phi$ of gold or silver. It shows that for a single metal crystal or metal thin film on a single metal crystal vibrational relaxation is promoted by a smaller energy gap between $E_{\mathrm{v}}\left(v, r_{\text {outer }}\right)$ and $\Phi$. For $\left(E_{\mathrm{v}}\left(v, r_{\text {outer }}\right)-\Phi\right)<-5 \mathrm{eV}$ almost no vibrational relaxation can be observed while for $\left(E_{\mathrm{v}}\left(v, r_{\text {outer }}\right)-\Phi\right)>-3 \mathrm{eV}$ almost no molecule stays in its initial vibrational state. The incident translational energy in all experiments was around $E_{\text {inc }} \approx 0.5 \mathrm{eV}$. Hypothetical points for $\mathrm{NO}(v=3)$ and $\mathrm{NO}(v=11)$ scattered from both $\mathrm{VO}_{2}(\mathrm{M})(\Phi=4.88 \mathrm{eV})$ and $\mathrm{VO}_{2}(\mathrm{R})(\Phi=4.70 \mathrm{eV})$ in green. For $\mathrm{VO}_{2}(\mathrm{M})$ two points for each $\mathrm{NO}$ vibrational state are shown. The one with the higher relaxation probability assumes the metallic behavior in both $\mathrm{VO}_{2}(\mathrm{M})$ and $\mathrm{VO}_{2}(\mathrm{R})$ that follows the sigmoidal from the Au and Ag scattering experiments. The difference between $\mathrm{VO}_{2}(\mathrm{M})$ and $\mathrm{VO}_{2}(\mathrm{R})$ is based on their different work function. The other assumes that $\mathrm{VO}_{2}(\mathrm{M})$ shows insulator behavior and has a vibrational relaxation probability of 0. Adapted from Reference [15].

the $\mathrm{NO}(v=11)$ scattering experiments from silver and gold in the thesis of Bastian Krüger 11 . If the gap is large enough $\left(\left|\Phi-E_{\mathrm{v}}\left(v, r_{\text {outer }}\right)\right|>6 \mathrm{eV}\right)$ almost no relaxation takes place.

As this work is concerned with scattering vibrationally excited $\mathrm{NO}$ from $\mathrm{VO}_{2}$ films it is of interest to know how this system fits into this picture. Besides the previous experiments with gold and silver surfaces Figure 2.13 also shows hypothetical points for the relaxation probability of $\mathrm{NO}(v=3)$ and $\mathrm{NO}(v=11)$ scattered from $\mathrm{VO}_{2}$. The work 


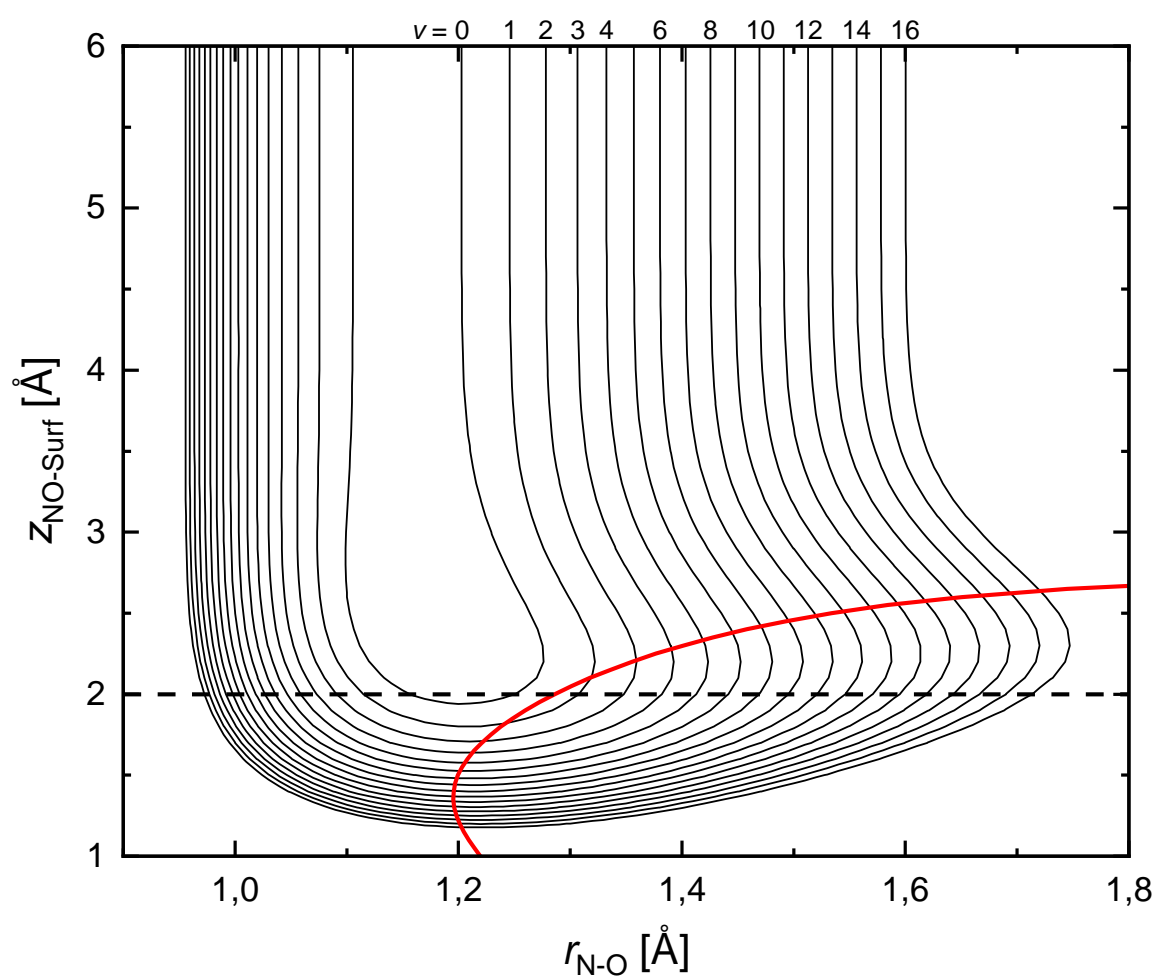

Figure 2.14: Contour plot of the adiabatic potential energy surface of ground state NO over a $\mathrm{Au}(111)$ surface hollow site. The potential energy surface was calculated following the work of Roy et. al. 57 . The contour lines indicate the energy of the vibrational states $v=0$ to $v=16$. The red line indicates the crossing curve between the diabatic energy surfaces of $\mathrm{NO} \mathrm{X}^{2} \Pi_{\mathrm{r}}$ and $\mathrm{NO}^{-} \mathrm{X}^{3} \Sigma^{-}$and therefore marks the line where the NO transforms from neutral to anionic particle along the adiabatic potential. The dashed line mark the potential energy curve depicted in Figure 2.15. Adapted from Reference [58.

function $\Phi$ of $\mathrm{VO}_{2}$ is taken to be $4.88 \mathrm{eV}$ for the low conducting $\mathrm{VO}_{2}(\mathrm{M})$ and $4.70 \mathrm{eV}$ for the high conducting $\mathrm{VO}_{2}(\mathrm{R}) \underline{60}$. Note that the literature is contradictory concerning the work function values 61 . The vibrational energy at the outer turning point $E_{\mathrm{v}}\left(v, r_{\text {outer }}\right)$ for $\mathrm{NO}(v=3)$ and $\mathrm{NO}(v=11)$ on the other hand is taken from the previous works $\frac{15}{15}$ where it was scattered from gold or silver.

According to the fact that $\mathrm{VO}_{2}$ is a Peierls insulator it should show the usual metal behavior like gold or silver when the surface temperature is above the MIT. Therefore the points for $\mathrm{NO}$ scattering from $\mathrm{VO}_{2}(\mathrm{R})$ are placed on the sigmoidal from the scattering experiments with gold and silver. $\mathrm{VO}_{2}(\mathrm{M})$ present below the MIT temperature can be assumed to also show metallic behavior. In this case the difference in vibrational relaxation probability between both $\mathrm{VO}_{2}$ phases is based solely on the work function difference. On the other hand $\mathrm{VO}_{2}(\mathrm{M})$ could also show insulator behavior where the re- 


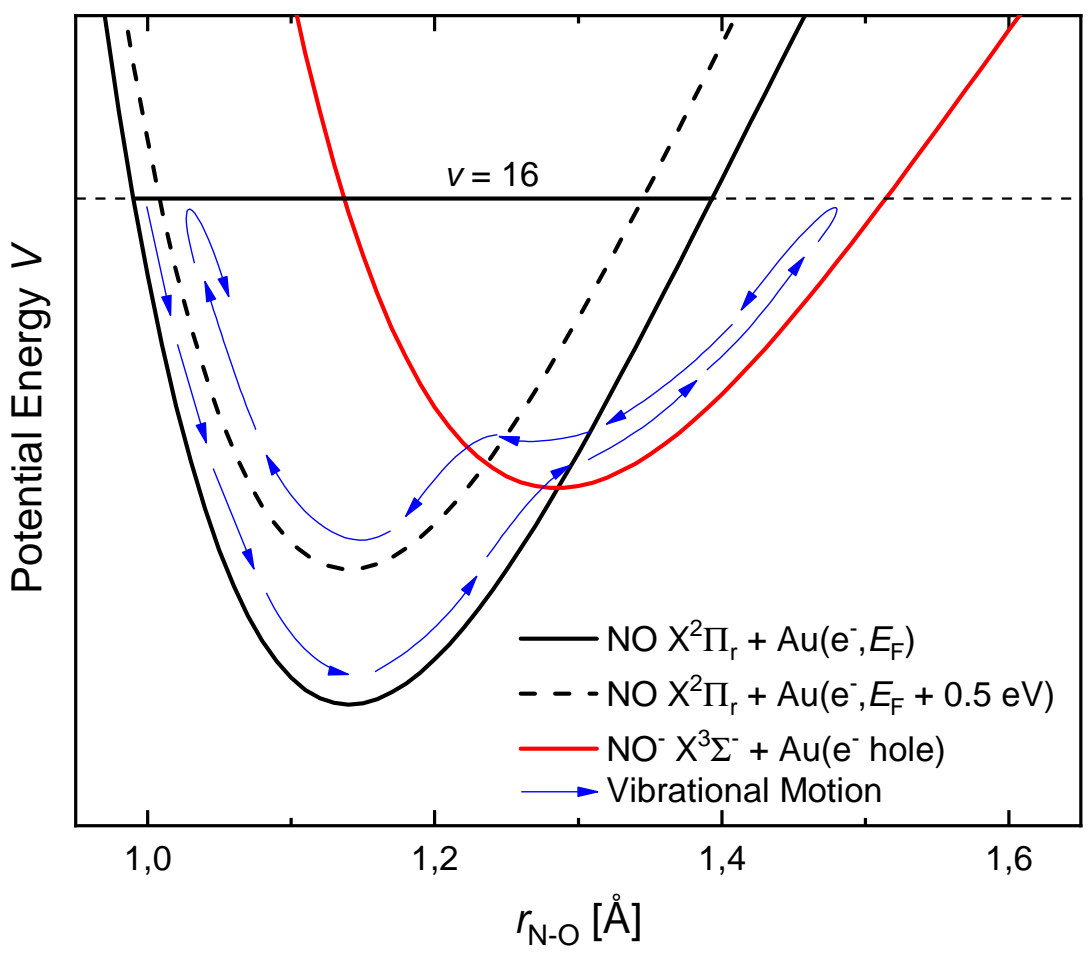

Figure 2.15: Potential energy curves for $\mathrm{NOX}^{2} \Pi_{\mathrm{r}}$ (black) and $\mathrm{NO}^{-} \mathrm{X}^{3} \Sigma^{-}$(red) placed at a distance of $z=2 \AA$ above a gold surface. The curves correspond to the potential energy surface shown in Figure 2.14 along the dashed line. From these curve the mechanism of nonadiabatic energy transfer from the molecular vibrational motion (blue arrows) of NO to the gold surface can be seen. Starting from the inner turning point of the $\mathrm{NOX}^{2} \Pi_{\mathrm{r}}$ potential at $\approx 1 \AA$ the molecule stretches until the potential energy curve of $\operatorname{NOX}^{2} \Pi_{\mathrm{r}}$ crosses the potential energy curve of $\mathrm{NO}^{-} \mathrm{X}^{3} \Sigma^{-}$. Here, similar to Figure 2.11 the molecule transforms from the neutral state to the anionic state by extracting an electron from the Au surface. This process occurs with a finite probability. From here it follows the potential energy curve of $\mathrm{NO}^{-} \mathrm{X}^{3} \Sigma^{-}$ to the outer turning point at $\approx 1.5 \AA$. On the way back the molecule takes the non-adiabatic path and misses the potential energy curve of $\mathrm{NOX}^{2} \Pi_{\mathrm{r}}$. It continues to the dashed line which represents the potential energy curve of $\mathrm{NOX}^{2} \Pi_{\mathrm{r}}$ elevated by $0.5 \mathrm{eV}$ where again it transforms back to neutral $\mathrm{NOX}^{2} \Pi_{\mathrm{r}}$. Since $\mathrm{SI} 0.5 \mathrm{eV}$ are now transferred to the surface the vibrational motion of $\mathrm{NOX}^{2} \Pi_{\mathrm{r}}$ is reduced from the initial $v=16$. Adapted from Reference [58].

laxation probability becomes almost zero. The difference between $\mathrm{VO}_{2}(\mathrm{M})$ and $\mathrm{VO}_{2}(\mathrm{R})$ would be much smaller in the first case than in the second case but should still easily observable.

To explain this phenomenon one has first to understand the mechanism of vibrational relaxation in molecule-metallic surface scattering. Figure 2.14 shows the calculated adiabatic potential energy surface of a ground state NO molecule above a hollow site of a $\mathrm{Au}(111)$ as function of the $\mathrm{N}-\mathrm{O}$ distance $r_{\mathrm{N}-\mathrm{O}}$ and the molecule-surface distance 
$z_{\mathrm{NO}-\text { Surf }}$. The red line marks the transition between the neutral and the anionic character of $\mathrm{NO}$ with the latter being more present at long N-O distance and close to the surface. It can be seen that with increasing proximity to the surface longer N-O distances become more stable. In consequence this leads to a situation where as long as the NO is sufficiently close to the surface it phases between the neutral and anionic state as it stretches and contracts in its vibrational motion by exchanging an electron back and forth with the surface as shown in Figure 2.15. While the adiabatic picture is due to the small kinetic energy of translational motion dominant along the molecule-surface distance the non-adiabatic picture is more valid along the N-O axis. So with every crossing from neutral to anionic state there is a finite chance for the exchanged electron to end up on a different energy level.

This is schematically shown in Figure 2.15 where the vibrational motion depicted as blue arrows start at the inner turning point of the potential energy curve of $\operatorname{NOX}^{2} \Pi_{\mathrm{r}}(v=16)$ depicted as a solid black line. Along the vibrational motion the molecule stretches and phases adiabatically to $\mathrm{NO}^{-} \mathrm{X}^{3} \Sigma^{-}$by abstracting a surface electron depicted by the solid red curve where it hits the outer turning point of the anionic potential and is contracted again. Instead of following the adiabatic potential back to the initial state it performs a non-adiabatic transition and continues on the anionic potential. From here it takes the route unto the dashed potential of $\mathrm{NOX}^{2} \Pi_{\mathrm{r}}$ by transferring the electron back to an excited state in the surface. In consequence, the potential energy curve of $\mathrm{NOX}^{2} \Pi_{\mathrm{r}}$ is shifted along the $\mathrm{y}$-axis by the same amount of energy as the difference between the surface's ground and excitated state. In this case the shift is $0.5 \mathrm{eV}$. As the available vibrational energy for the elevated potential energy curve of the molecule stays the same the effective vibrational quantum is reduced. This process can occur in both direction ergo reducing and increasing the vibrational excitation of the molecule but since the for the mechanism relevant non-adiabatic transition is velocity dependent it occurs preferred in higher vibrational states with faster vibrational motion so that in consequence relaxation is more probable.

The relative energetic distance between the potential energy curve of $\mathrm{NO}^{-} \mathrm{X}^{3} \Sigma^{-}$and the potential energy curve of $\operatorname{NOX}^{2} \Pi_{\mathrm{r}}$ is determined by both the work function $\Phi$ of the surface, the vertical binding energy at the molecules outer turning point $E_{\mathrm{v}}\left(v, r_{\text {outer }}\right)$ and the image charge stabilization (ICS) the surface exerts on the ion. A higher work function shifts the curve to a higher energy because more energy is required to abstract an electron from the surface and to form the anion. A higher vertical binding energy at the outer turning point shifts the anionic potential energy curve relative to the neutral potential energy curve to lower energies. Image charge stabilization lowers also the anionic energy curve the anionic state is stabilized in proximity to the metallic surface. 
A lower anionic potential energy curve means that the crossing point between the two potential energy curves lies closer to the minimum of the vibrational potential. Here, more kinetic energy in the vibrational motion is present which increases the probability of a non-adiabatic transition in accordance with Equation 2.36 . 



\section{Experimental Setup}

\subsection{Home-Built UHV Compatible Four-Terminal Sensing Device}

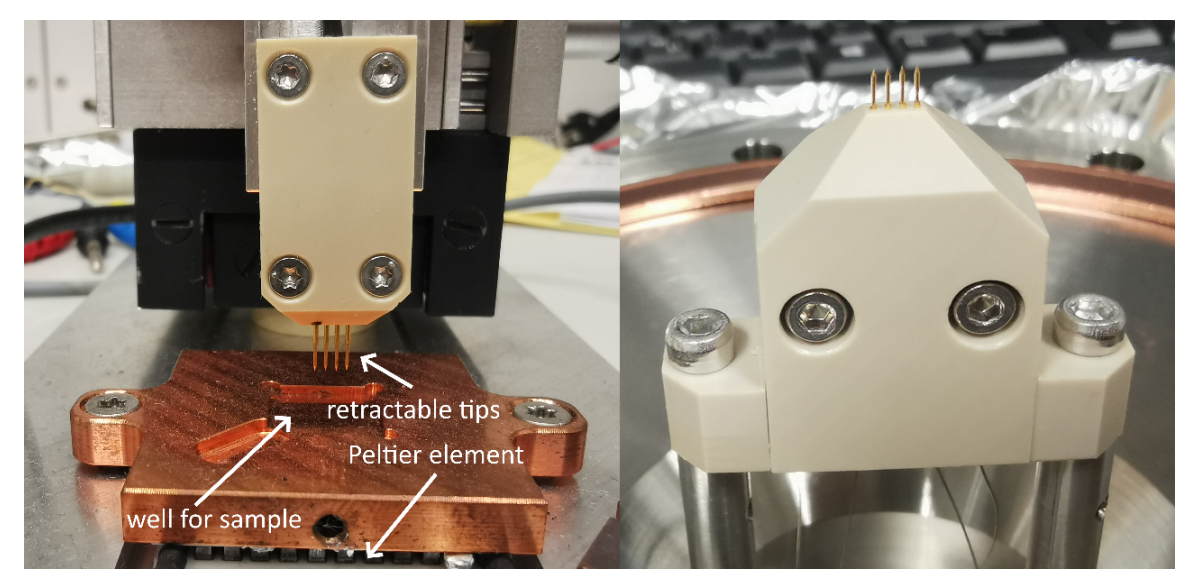

Figure 3.1: Images of Four-Terminal Sensing for measurements under atmosphere (left) and for measurements in UHV conditions (right).

As mentioned above in Section 2.1 the metal-to-insulator transition at $68{ }^{\circ} \mathrm{C}$ is accompanied by a resistance change of several orders of magnitude in the $\mathrm{VO}_{2}$ film. The resistance of a substance can be accurately probed by using Four-Terminal Sensing, which is in the following referred to as 4-Probe. For this a device was built that allows to approach a surface with four spring-loaded contacts (Feinmetall, Standard Probe F701.18) that are arranged in a row with $1 \mathrm{~mm}$ spacing. The ensemble can be moved along three dimensions with micrometer screws. The voltage and current is simultaneously measured and applied by a multimeter (Keithley, 2400 Source Meter). To compensate for the voltage offset caused by different surface potentials between the contacts and the sample surface a compensation measurement was done. At the beginning of each measurement the source is set to zero. The measured zero current $I_{0}$ and 
zero voltage $U_{0}$ were used to gain offset corrected resistance measurements as a function of the the surface temperature $T_{\text {Surf }}$ with the equation

$$
R\left(T_{\text {Surf }}\right)=\frac{U\left(T_{\text {Surf }}\right)-U_{0}}{I\left(T_{\text {Surf }}\right)-I_{0}} .
$$

The sample is placed in a solid copper block during the measurement. The copper can be heated and cooled by a $100 \mathrm{~W}$ Peltier element which is used to ramp the sample temperature linearly. The temperature is measured with a K-Type thermocouple read by voltmeter (National Instruments, NI-9212). The end of the thermocouple is positioned in the center of the copper block which allows a repetition rate of $10 \mathrm{~Hz}$. The measurement is controlled and processed by a home-built LabVIEW program (National Instruments, LabVIEW 2014).

A similar four-terminal sensing probe was build from UHV-compatible materials and attached to a DN $40 \mathrm{CF}$ BNC feed-through (Pfeiffer Vacuum). The contacts used are the same as mentioned above (Feinmetall, Standard Probe F701.18) also with a $1 \mathrm{~mm}$ spacing to allow comparability.

\subsection{Preparation of $\mathrm{VO}_{2}$ films}

In contrast to the pure metals used in previous $\mathrm{NO}$ scattering experiments 4143 vanadium dioxide is not commercially available as cut single crystals. This is because bulk crystals of $\mathrm{VO}_{2}$ cannot be grown. It is only stable as a film on a support substrate.

To prepare vanadium dioxide surfaces for experimental analysis a number of methods can be used such as chemical vapor deposition (CVD), radio frequency magnetron sputtering (RFMS), laser ablation or sol gel deposition. All this methods have in common that they produce films of varying thickness but no self standing crystals. This work uses samples made by CVD employing $\mathrm{V}(\mathrm{acac})_{2}$ as precursor and RFMS of $\mathrm{V}_{2} \mathrm{O}_{3}$. The specific methods are described in detail in the following sections.

\subsubsection{Chemical Vapor Deposition}

Chemical vapor deposition is chosen for the preparation of vanadium dioxide film because it is a technique with high throwing power which in general is able to generate uniformly thick depositions on a variety of substrate materials. In contrast to other techniques, like laser ablation or radio frequency sputtering, it does not require high vacuum conditions or expensive laser setups. Furthermore, CVD is not restricted to the line of sight making it possible to line up several substrates in each experiment. This makes CVD a good candidate for the cheap and quick production of samples for surface 
scattering experiments. Earlier works have already shown that CVD is suitable for the formation of the desired $\mathrm{VO}_{2}(\mathrm{M})$ species. 62,63

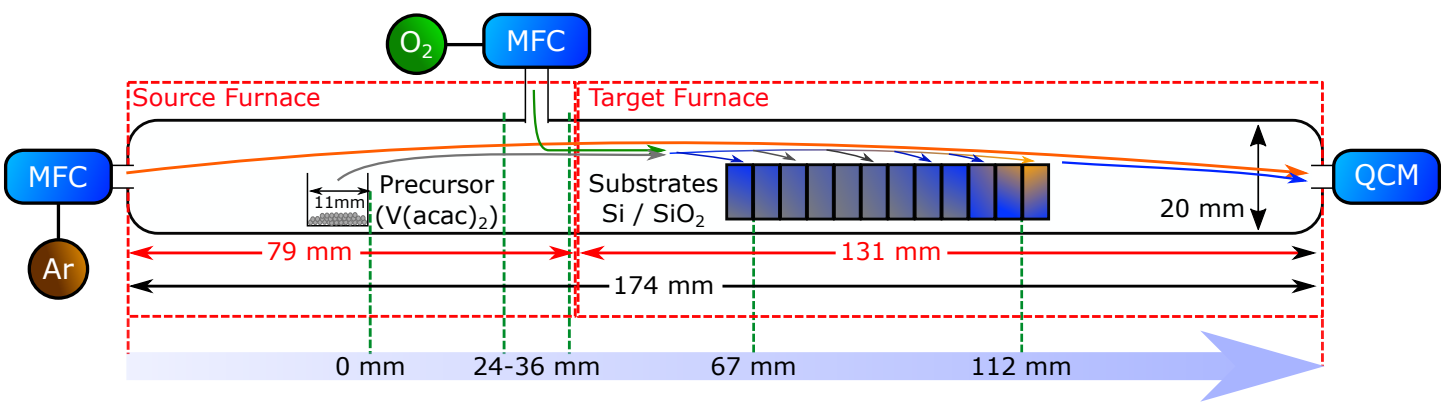

Figure 3.2: Schematic sketch of the chemical vapor deposition setup. The precursor $\mathrm{V}(\mathrm{acac})_{2}$ is placed in a crucible at the front side of the quartz tube. It is heated by the source furnace which encloses the left half of the quartz tube. From here the vaporized precursor is dragged by a stream of argon gas from the mass flow controller (MFC) at far left end of the tube in the direction of the substrate plates. Along the way oxygen gas is added from another MFC to oxidize the precursor to vanadium oxide. The substrate plate holder is placed in the right half of the tube which itself is enclosed by the target furnace. One part of the vanadium dioxide deposits on the substrate plates and the rest is dragged further to the far right end of the tube. There it is caught by a quartz crystal microbalance which is used to detect if $\mathrm{VO}_{2}$ is produced. The gas flows are indicated by colored arrows. Orange for argon, green for oxygen, grey for $\mathrm{V}(\mathrm{acac})_{2}$ and blue to orange for vanadium oxides.

For the samples used in this work the home-built setup shown in Figure 3.2 was used. It was set up and operated by Anita Pilipodi-Best in Tel Aviv. It consists of a $174 \mathrm{~mm}$ long and $20 \mathrm{~mm}$ inner diameter fused quartz tube with mass flow controlled (MFC) inlets for argon and oxygen in the front region and an outlet with a quartz crystal microbalance (QCM) on the backside. The former allows a controllable gas flow along the tube while the latter makes it possible to detect changes in the composition of the exiting gas flow. The front part of the tube contains a precursor reservoir with vanadium acetylacetone powder $43 \mathrm{~mm}$ behind the argon inlet. The rear part of the tube contains the substrate holder with up to $1210 \mathrm{~mm} \times 10 \mathrm{~mm}$ substrate wafers positioned $67 \mathrm{~mm}$ to $112 \mathrm{~mm}$ downstream from the precursor. Both parts are enclosed by two separate furnaces to allow individual temperature control of the precursor and substrate section. The precursor or source furnace can be heated up to $260^{\circ} \mathrm{C}$ to allow the controlled sublimation of the $\mathrm{V}(\mathrm{acac})_{2}$ precursor $\left(T_{\text {subl }} \approx 277^{\circ} \mathrm{C}\right.$ at $\left.1 \mathrm{bar}\right)$ in the argon gas stream. Usual temperatures for the sublimation are $175^{\circ} \mathrm{C}$ to $190^{\circ} \mathrm{C}$. The substrate or target furnace can maintain temperatures up to $1200^{\circ} \mathrm{C}$ with most depositions conducted at $370{ }^{\circ} \mathrm{C}$ to $600{ }^{\circ} \mathrm{C}$. The experiment is usually conducted at pressures ranging from 20 Torr to 100 Torr. 


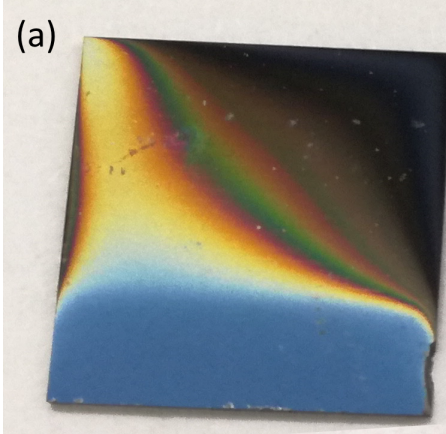

(d)

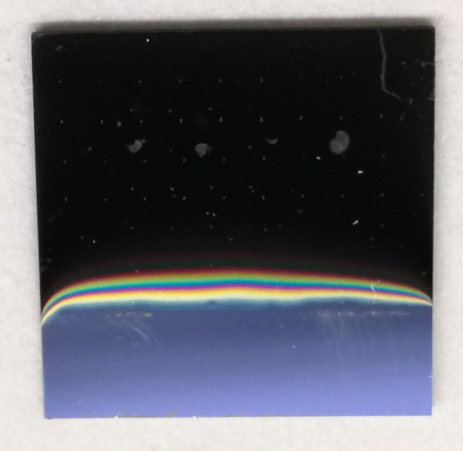

(b)

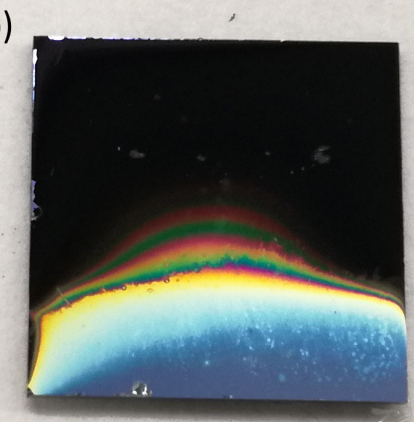

(e) (c)
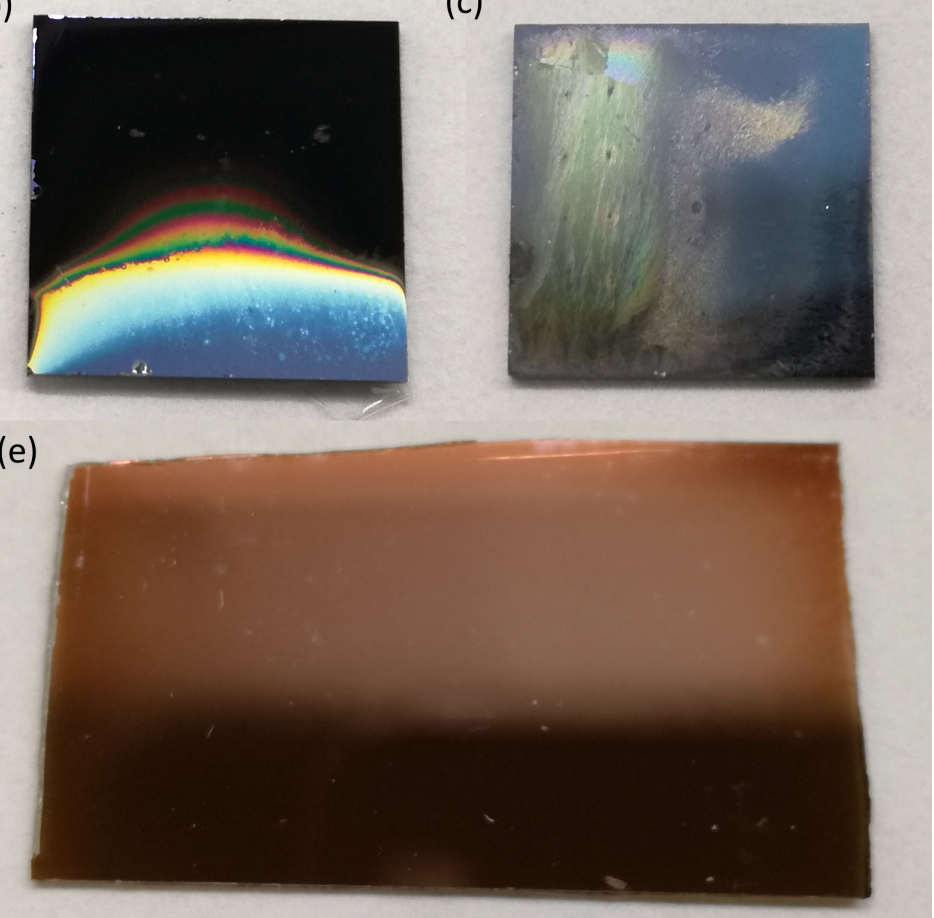

Figure 3.3: Images of various $\mathrm{VO}_{2}$ films produced by CVD (a-d) and reactive radio frequency magnetron sputtering (e). (a) and (b) show only partially covered substrates. The colorful sections are thinly covered while the black areas are thicker. Such depositions occur when the substrate is placed to close to the precursor crucible or if the gas flow is not strong enough. (c) shows a surface that is oxidized too far and covered with yellow crystalline $\mathrm{V}_{2} \mathrm{O}_{5}$. (d) shows a surface which is evenly covered by matt black $\mathrm{VO}_{2}$. The blue shiny section is uncovered substrate. (e) shows a slab of r-cut sapphire covered by a thin film of $\mathrm{VO}_{2}$ through reactive radio frequency magnetron sputtering. The color stems from reflections of the surroundings. The surface is due to its thickness actually colorless and reflective.

Experiments at the Open University of Israel in Tel-Aviv showed that it is possible to deposit vanadium dioxide onto silicon wafer substrates with a sufficiently intense metalto-insulator phase transition between $65^{\circ} \mathrm{C}$ to $70^{\circ} \mathrm{C}$. A resistance change over several orders of magnitude was measured with multiple samples prepared that way. It also showed that the results of CVD deposition rely heavily on the CVD parameters such as temperature program, gas flow rate, pressure, $\mathrm{Ar}-\mathrm{O}_{2}$ ratio deposition time and distance between precursor container and substrate. While this can be used to produce a variety of different grain sizes, oxidation states and structures of vanadium oxides it makes it hard to find the conditions necessary for the desired high order, uniformly covered $\mathrm{VO}_{2}(\mathrm{M})$ films. A selection of different results from the CVD preparation is shown in Figure 3.3 (a-d). 
Furthermore, parameters of earlier reports on $\mathrm{VO}_{2} \mathrm{CVD}$ deposition rarely give the same results on a different setup. Different geometries lead to different local temperatures and flow rates on the substrates surface even if the same set-points are used. Since local temperatures and flow rates are hard to determine these are rarely reported and have to be investigated from scratch for a new apparatus. Also, the prepared films have always either a grainy structure or show only an insufficiently intense MIT. Therefore, part of this work is concerned with the fact if direct scattering of NO is possible from these rough surfaces or if all scattered molecules become entrapped upon collision.

\subsubsection{Reactive Radio Frequency Magnetron Sputtering}

The second technique used for $\mathrm{VO}_{2}$ preparation is reactive radio frequency magnetron sputtering. This is a physical vapor deposition (PVD) technique. The preparation was performed by the group of Amos Sharoni at the Bar Ilan University in Israel 64. It requires a target surface, in this case made of $\mathrm{V}_{2} \mathrm{O}_{3}$, which is sputtered at $600{ }^{\circ} \mathrm{C}$ with an $\mathrm{Ar} / \mathrm{O}_{2}$ plasma. The atmosphere consists of $2 \% \mathrm{O}_{2}$ and $98 \% \mathrm{Ar}$ with a pressure of $3 \mathrm{~m}$ Torr. The surface was deposited for $100 \mathrm{~min}$. Assuming a deposition rate of $1 \mathrm{~nm} \min ^{-1}$ we can assume that the samples have an approximate thickness of about $100 \mathrm{~nm}$. An example of such a thin film is shown in Figure 3.3 (e).

\subsubsection{Preparation of $\mathrm{VO}_{2}$ under UHV conditions for Surface Scattering}

Surface preparation for experiments under UHV conditions are not trivial and require different procedures of ion-sputtering, annealing in various atmospheres and to different temperatures 65 . This can be even more complicated regarding composite substances such as $\mathrm{VO}_{2}$ where the different cleaning procedure may not only influence the physical form of the surface but also its chemical composition. This might lead to the immediate destruction of the surface or might change properties relevant for the subsequent experiments.

The chemical composition of the $\mathrm{VO}_{2}$ surface is monitored with Auger Electron Spectroscopy (AES). The electronic characteristics of the surface are monitored with a fourterminal sensing device (4-Probe). Surface preparation was incidentally performed using the cleaning procedure known from gold and silver scattering experiments. Here, the surface is first sputtered with $\mathrm{Ar}^{+}$-ions with $1 \mathrm{kV}$ acceleration and subsequently annealed. Quickly, it became clear that heating beyond $150{ }^{\circ} \mathrm{C}$ for less than 5 min already reduces the resistance of $\mathrm{VO}_{2}(\mathrm{M})$ permanently by several orders of magnitude. In most cases, the resistance of the $\mathrm{VO}_{2}(\mathrm{R})$ phase also shifts slightly. This is illustrated in 


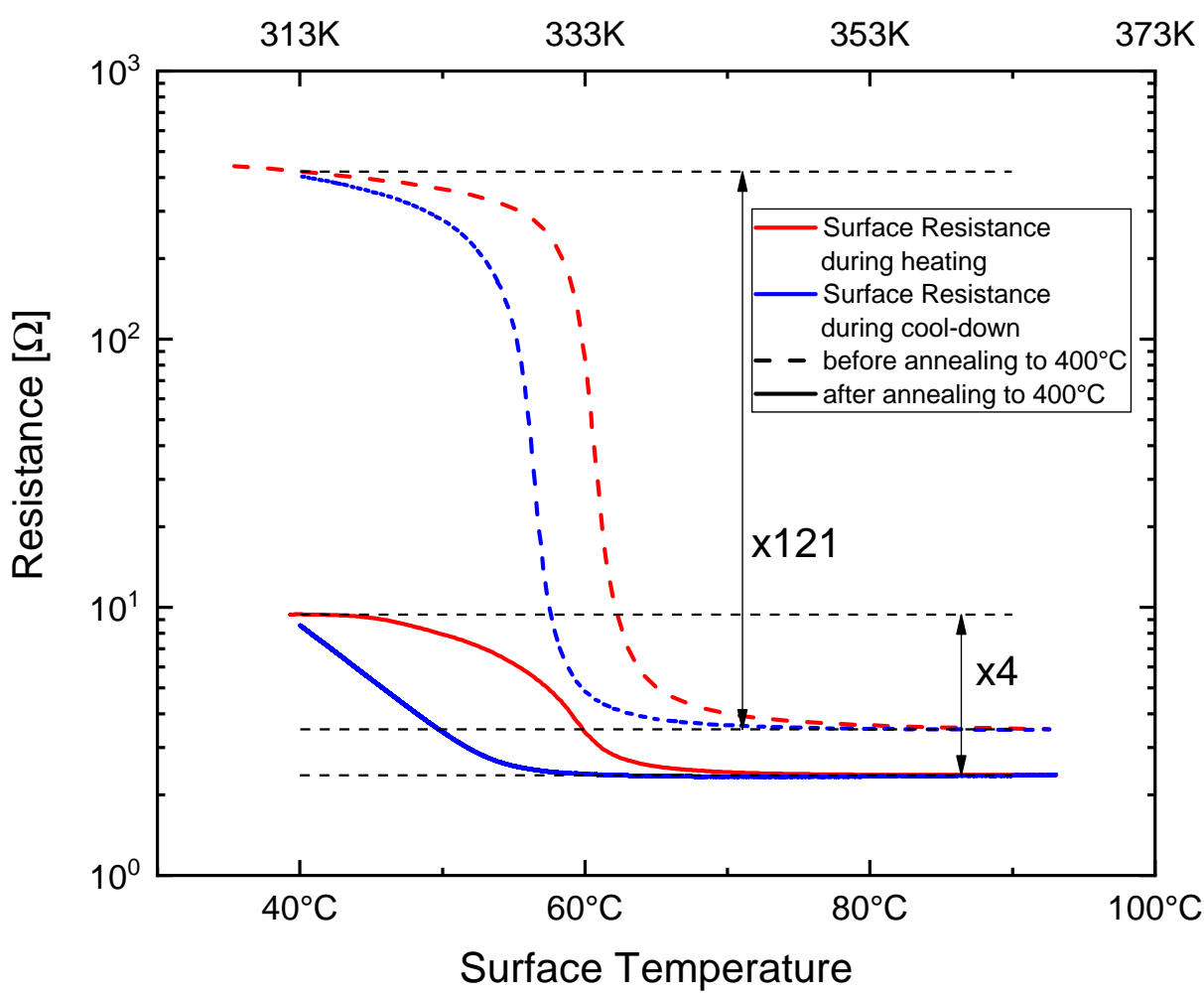

Figure 3.4: Resistance measurement over the surface temperature of a CVD prepared $\mathrm{VO}_{2}$ sample (CVD3) before and after it was annealed to $400{ }^{\circ} \mathrm{C}$ for $30 \mathrm{~min}$.

Figure 3.4. It shows the resistance of a $\mathrm{VO}_{2}$ film as a function of surface temperature before and after heating it for $30 \mathrm{~min}$ to $400^{\circ} \mathrm{C}$. To break down the intensity of the MIT to a single number the term "MIT contrast" will be used in the following. It consists of the ratio of the $\mathrm{VO}_{2}$ surface resistance at $40{ }^{\circ} \mathrm{C}$ and the $\mathrm{VO}_{2}$ surface resistance at $90^{\circ} \mathrm{C}$. The MIT contrast of the sample shown in Figure 3.4 is reduced by three orders of magnitude from 120 to 4 .

Later surface preparations refrained from heating the surface beyond what is necessary to measure the MIT contrast. Therefore, the surface was kept below $100^{\circ} \mathrm{C}$ at all times. While it is thinkable that annealing might induce some surface reconstruction effect in the $\mathrm{VO}_{2}$ surface and evaporate gaseous surface contaminations the conservation of MIT contrast has a higher priority.

While $\mathrm{Ar}^{+}$-ion sputtering is less destructive than heating, it also reduces the MIT contrast. The black points in Figure 3.5 show how subsequent $\mathrm{Ar}^{+}$-ion sputtering reduces the measured MIT contrast in the initial step from about 10000 down to 60 after 5 min of sputtering. After subsequent sputtering sessions the MIT contrast saturates to a value of 30 . While this may sound at first that $\mathrm{Ar}^{+}$-ion sputtering has a more severe 


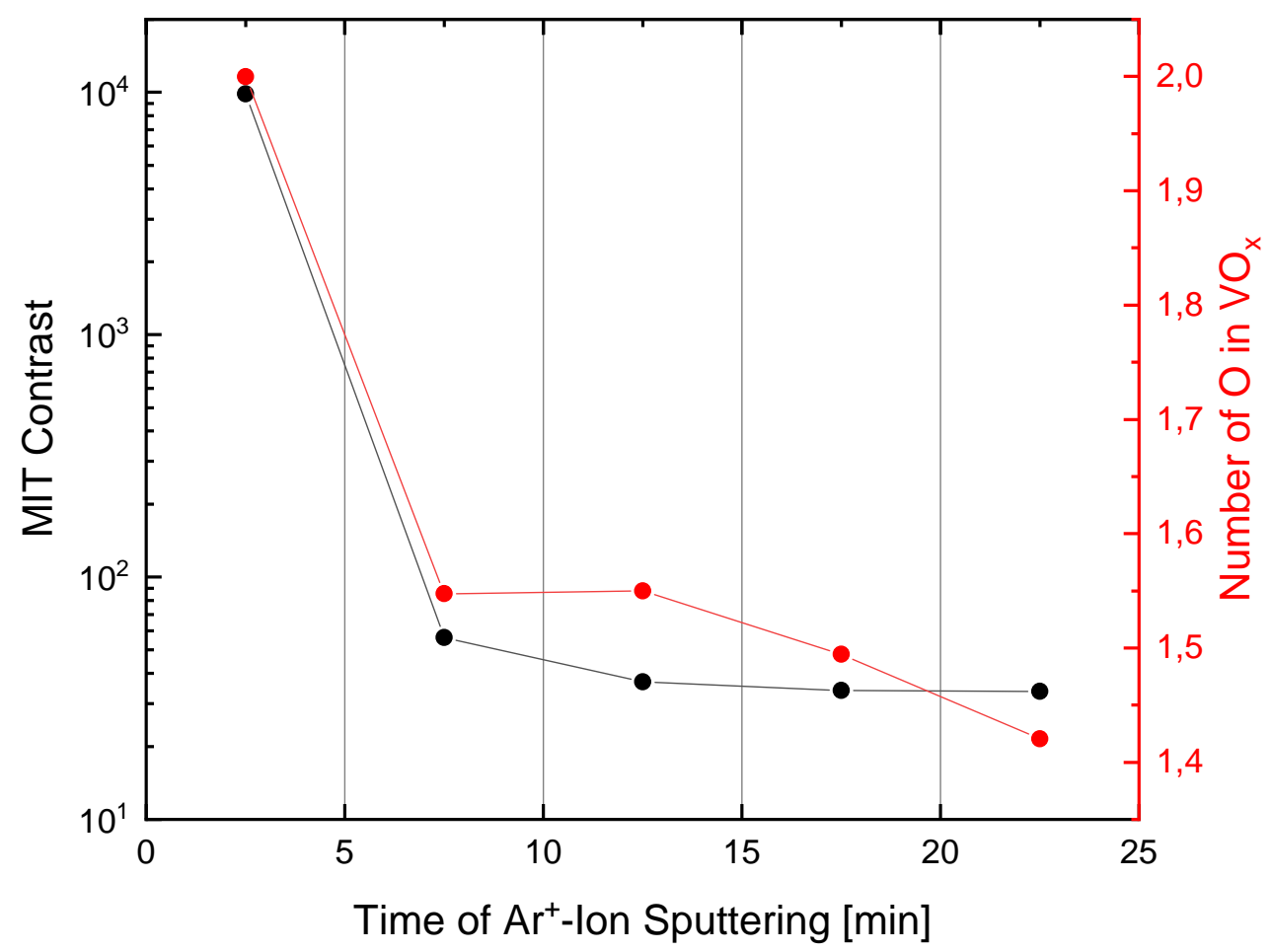

Figure 3.5: Influence of subsequent 5 min sputtering sessions with $1 \mathrm{kV}$ accelerated $\mathrm{Ar}^{+}$ions on the MIT contrast (black points) and oxygen-to-vanadium ratio measured by Auger Electron Spectroscopy (red points).

impact on the MIT contrast than heating it should be noted that for scattering experiments the final MIT contrast after preparation is relevant and not the overall loss of MIT contrast. Heating always results in a MIT contrast of 5 or less. $\mathrm{Ar}^{+}$-ion sputtering results in MIT contrasts between 10 and 1000, depending on the sample and the time of sputtering.

The negative influence of $\mathrm{Ar}^{+}$-ion sputtering is also shown with AES. The intensity of a given AES signal in a spectrum is proportional to the amount of the element found in the probed sample. By taking into account the Auger sensitivity factor it is possible to get a rough estimate of the surface's elemental composition [6]. The red points of Figure 3.5 show the ratio between the oxygen and vanadium signal normalized to 2 for the unsputtered surface. It can be seen that with subsequent $\mathrm{Ar}^{+}$-ion sputtering the ratio shifts to lower values that indicate that the $\mathrm{VO}_{2}$ surface is reduced to a $\mathrm{VO}_{1.5}$ compound. The effect is most probably kinetic and not $\mathrm{Ar}^{+}$-ion specific since loss of MIT contrast has also been observed by another group when irradiating a $\mathrm{VO}_{2}$ surface with low doses of high energy uranium ions $\frac{67}{6}$. The loss of oxygen can be explained by the preferred expulsion of the more volatile oxygen compared to vanadium through the 


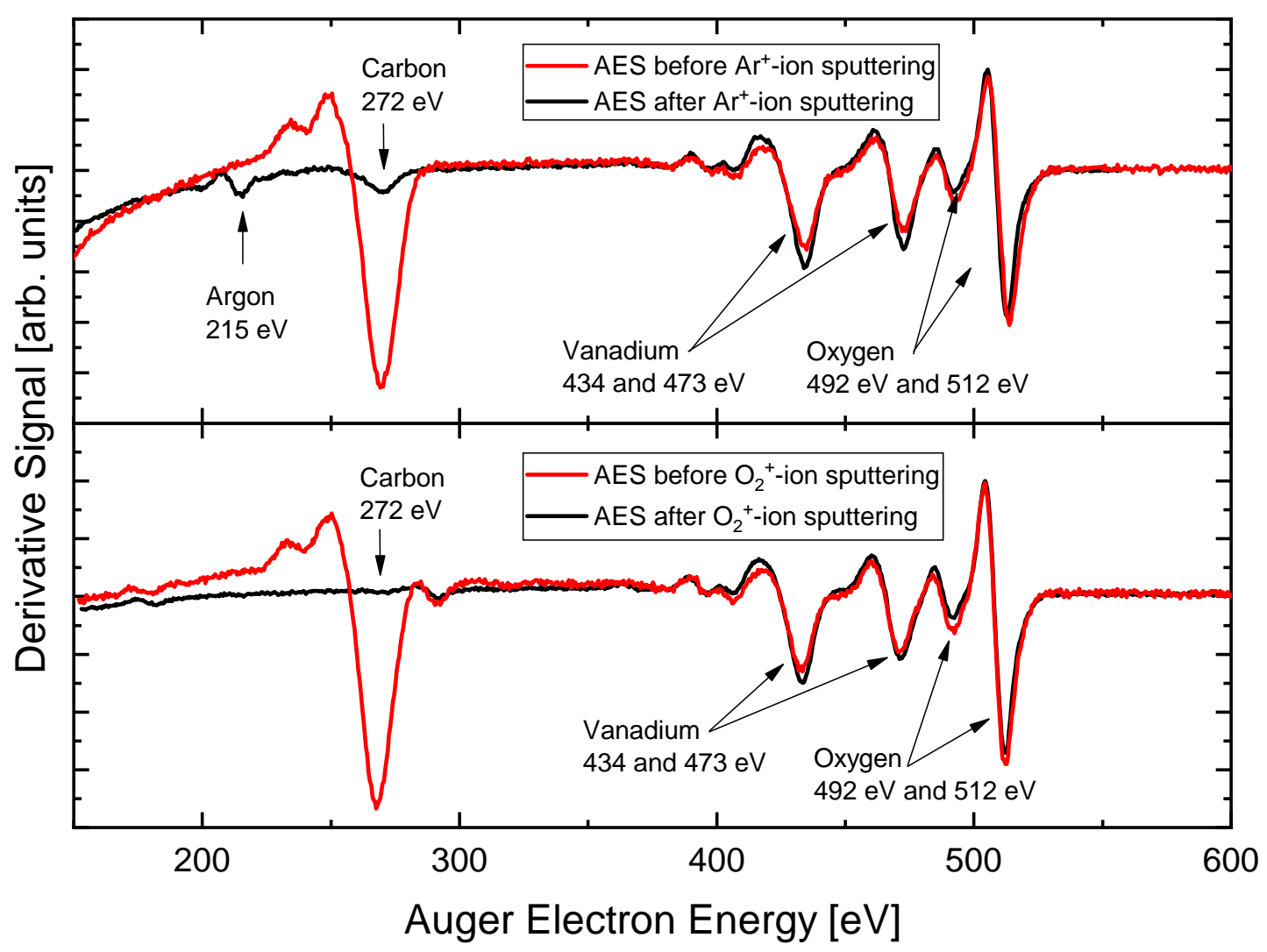

Figure 3.6: Auger Electron Spectra of a $\mathrm{VO}_{2}$ surface before (black) and after (red) $2 \times 10 \mathrm{~min}$ of $\mathrm{Ar}^{+}$-ion sputtering (top) and $3 \times 10 \mathrm{~min}$ of $\mathrm{O}_{2}^{+}$-ion sputtering (bottom) with $1 \mathrm{kV}$ acceleration.

kinetic impact of ions or the local heat up at the impact side. In any case this chemical reduction of vanadium dioxide is the most probable cause for the loss in MIT contrast during $\mathrm{Ar}^{+}$-ion sputtering. The similar shape of the black and red curve in Figure 3.5 suggests that the reduced vanadium oxide species is no longer able to perform the metal-to-insulator transition at $68^{\circ} \mathrm{C}$.

Furthermore, the main goal of sputtering is to remove foreign substances, mostly carbon, that stick to the surface. They are generated during the preparation procedure or by exposing the surface to the environment. For scattering experiments it is necessary to eliminate all these contaminations to make sure that the molecules scatter from the surface and not from a layer of undefined substances. Figure 3.6 (top) shows an AES spectra of a vanadium dioxide surface before and after sputtering with $\mathrm{Ar}^{+}$-ions for $4 \times 5 \mathrm{~min}$. A carbon signal can be seen at $272 \mathrm{eV}$ which is reduced to about 0.2 of its former height after sputtering. This means that the amount of $\mathrm{Ar}^{+}$-ion sputtering necessary to remove $80 \%$ of the carbon deposits on the surface will also reduce its MIT 


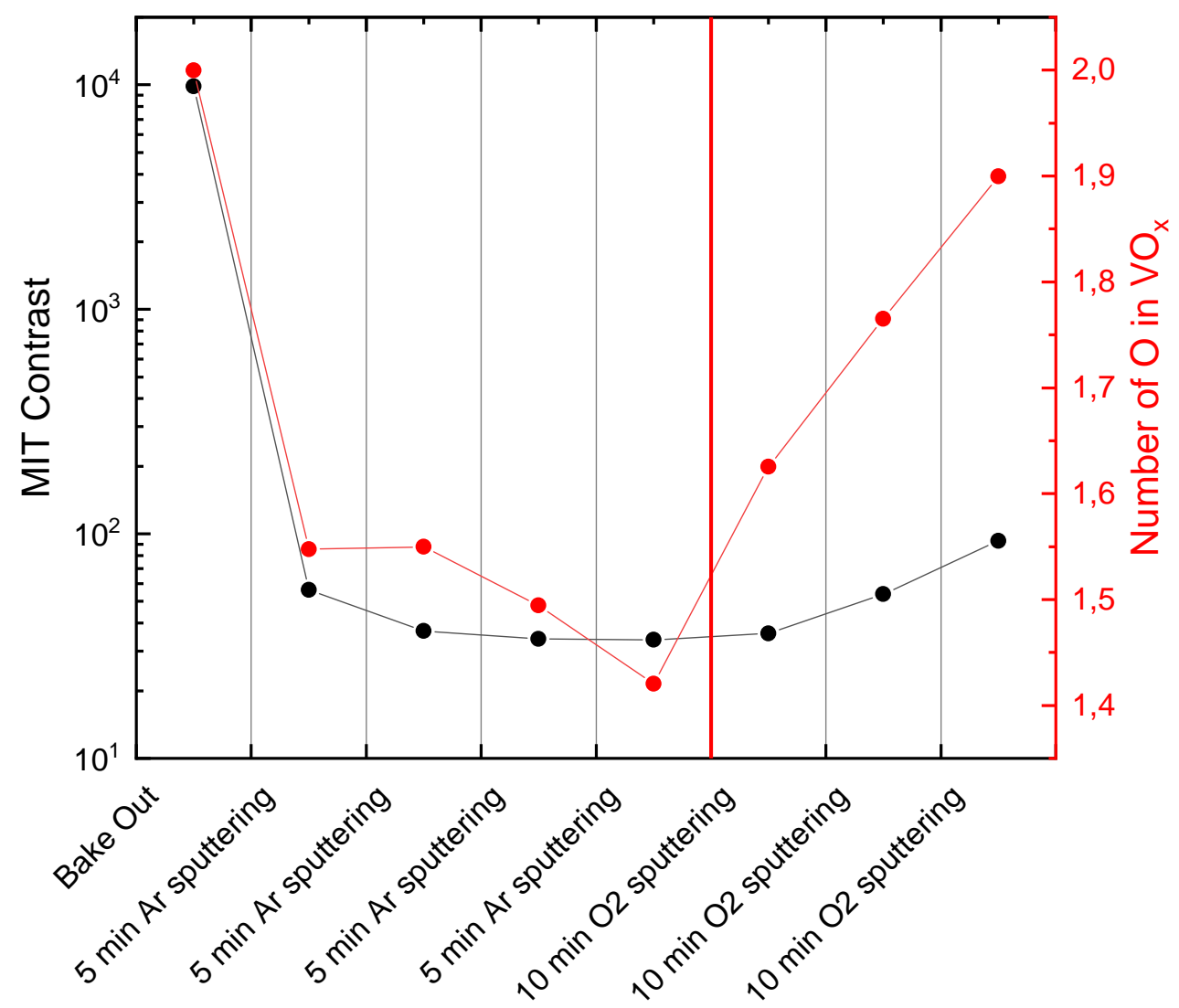

Figure 3.7: Influence of subsequent sputtering sessions with $1 \mathrm{kV}$ accelerated $\mathrm{Ar}^{+}$-ions or $\mathrm{O}_{2}{ }^{+}$-ions on the MIT contrast (black points) and oxygen-to-vanadium ratio measured by Auger Electron Spectroscopy (red points). The red vertical line indicates where the sputtering switches from $\mathrm{Ar}^{+}$to $\mathrm{O}_{2}{ }^{+}$-ion sputtering.

contrast by 2.5 orders of magnitude. This makes $\mathrm{Ar}^{+}$-ion sputtering unsuitable for the preparation of vanadium dioxide.

An alternative to sputtering with argon can be oxygen which is more reactive and able to oxidize carbon into gaseous species which are pumped out of the chamber. In Figure 3.6 (bottom) it can be seen that the carbon signal at $272 \mathrm{eV}$ is no longer visible in the AES after 30 min $\mathrm{O}_{2}{ }^{+}$-ion sputtering. This shows that oxygen is a viable option for the removal of carbon.

Usually, $\mathrm{O}_{2}{ }^{+}$-ions are not used as a sputter gas because the accelerated ions penetrate into the surface. In contrast to argon oxygen's reactive properties keep it bound to the sputtered material. This results in subsurface oxygen impurities which change the properties of a pure metal surface. The quality of a $\mathrm{VO}_{2}$ surface, which already contains oxygen, is less prone to suffer from oxygen insertion. Especially since the sputtering 


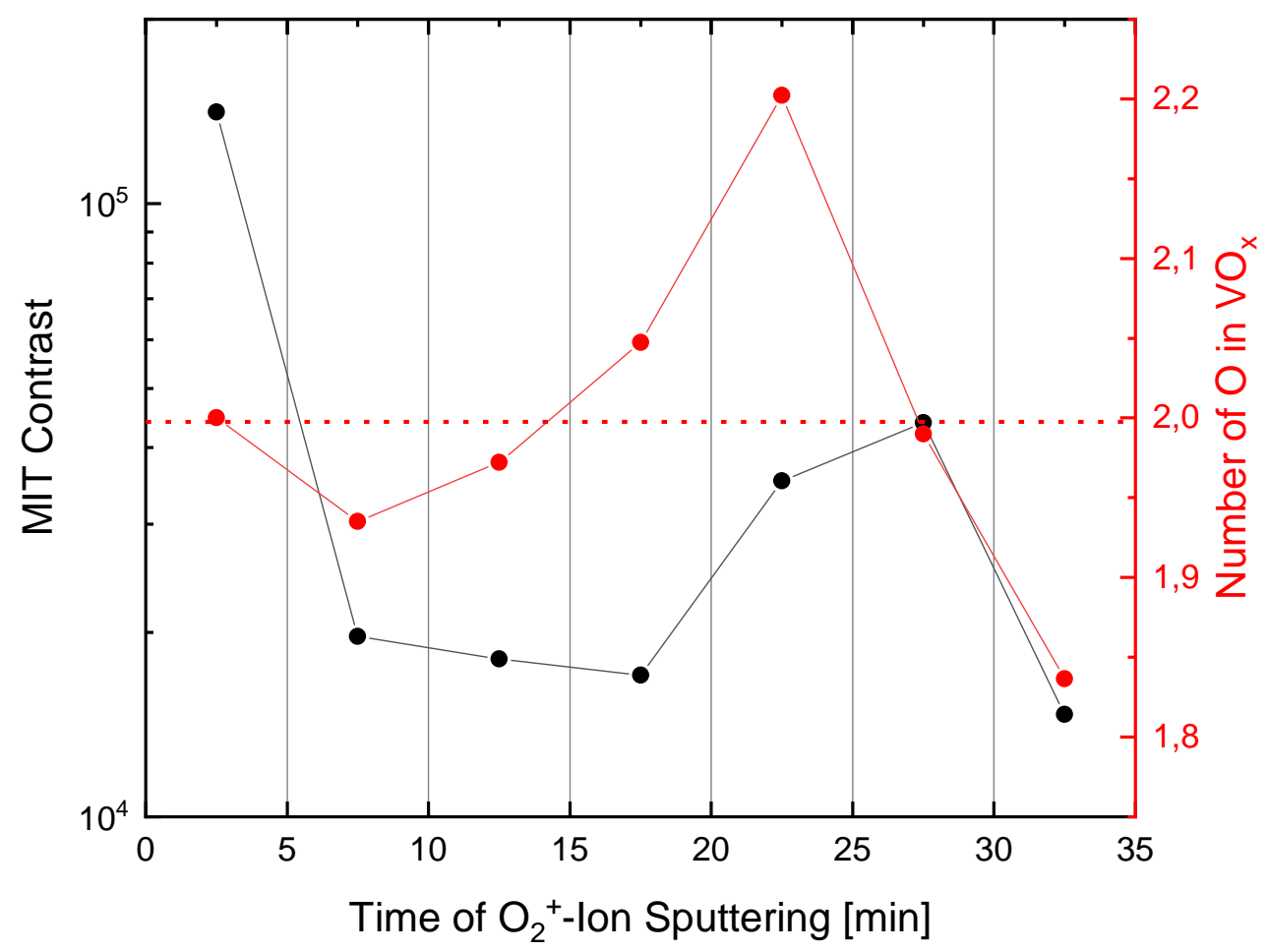

Figure 3.8: Influence of subsequent 5 min sputtering sessions with $1 \mathrm{kV}$ accelerated $\mathrm{O}_{2}{ }^{+}$ions on the MIT contrast (black points) and vanadium-to-oxygen ratio measured by Auger Electron Spectroscopy (red points). The red dotted line indicates the average value of all oxygen-to-vanadium ratios.

process itself appears to selectively remove oxygen from the $\mathrm{VO}_{2}$. Figure 3.7 shows that a previously by $\mathrm{Ar}^{+}$-ion sputtering depleted surface can be re-oxidized using $\mathrm{O}_{2}{ }^{+}$-ion sputtering. While the oxygen-to-vanadium ratio is almost back to 2 after $30 \mathrm{~min}$ of $\mathrm{O}_{2}{ }^{+}$sputtering the MIT contrast only rises to about 100 . This probably caused by the fact that although the oxygen in the surface is replenished the original $\mathrm{VO}_{2}$ structure is not automatically reconstructed. Therefore, the initial MIT properties are not regained. Figure 3.8 shows the influence of $\mathrm{O}_{2}{ }^{+}$-ion sputtering on a new $\mathrm{VO}_{2}$ surface. After an initial drop of less than one order of magnitude the MIT contrast saturates at values between 20000 and 40000 which exceeds everything accomplished with $\mathrm{Ar}^{+}$-ion sputtering by a factor of 100 . The oxygen-to-vanadium ratio shows no noticeable trend which suggest that the insertion of oxygen and removal of oxygen cancels each other out. Also the measured oxygen-to-vanadium ratios have on average the value 2 (dotted line). It can be assumed that the seen deviations are caused by measurement uncertainties and the amount of oxygen in the surface remains constant. 
It can be concluded that $\mathrm{O}_{2}{ }^{+}$-ion sputtering is the most viable option for surface preparation of $\mathrm{VO}_{2}$ under UHV conditions. $\mathrm{O}_{2}{ }^{+}$-ions have compared to argon ions a tolerable effect on the MIT contrast and surface composition and eliminate all detectable carbon contaminations.

\subsection{Laser Systems}

The preparation and detection of NO in various electronic, vibrational and rotational states is done by excitation with laser radiation in the ultraviolet, visible and infrared region of the electromagnetic spectrum generated by various laser systems described in the following section. Figure 3.9 shows the various transitions excited by electromagnetic radiation.

\subsubsection{Preparation of $\mathrm{NO}$ in $\mathrm{X}^{2} \Pi(v=3)$}

For the preparation of $\mathrm{NO} \mathrm{X}^{2} \Pi(v=3, J=0.5)$ from $\mathrm{NO} \mathrm{X}^{2} \Pi(v=0, J=0.5)$ overtone pumping by a high power nanosecond infrared system with nearly Fourier transform limited bandwidth was used [42]. It generates $1803.75 \mathrm{~nm}$ light with a bandwidth of $<0.001 \mathrm{~cm}^{-1}$. A schematic depiction of the laser system is shown in Figure 3.10 . A continuous wave (cw) ring dye laser (Sirah Matisse DR) is used to generate a narrow bandwidth $(<20 \mathrm{MHz})$ laser beam with a wavelength of $669.23 \mathrm{~nm}$ and a power of $0.5 \mathrm{~W}$. The ring dye laser is pumped with $5.5 \mathrm{~W} 532 \mathrm{~nm}$ laser light from a cw Nd:YLF laser (Coherent Verdi-10). The cw laser light is then amplified in a five stage pulsed amplifier (Sirah PulsAmp 5X), which is pumped by the second harmonic (532 nm) from an injection seeded pulsed Nd:YAG laser (Spectra Physics Quanta-Ray Pro-230). This generates $669.23 \mathrm{~nm}$ pulsed laser light with a $8 \mathrm{~ns}$ pulse duration and an energy up to $60 \mathrm{~mJ}$. This laser light is then used with $130 \mathrm{~mJ}$ of the fundamental $(1064 \mathrm{~nm})$ from the injection seeded pulsed Nd:YAG laser for difference frequency mixing (DFM) in a $\mathrm{LiNbO}_{3}$ crystal (Sirah DFM-2400-T). The generated $1803.75 \mathrm{~nm}$ light with energy of $3 \mathrm{~mJ}$ to $5 \mathrm{~mJ}$ is then again amplified by optical parametric amplification (OPA) in a second $\mathrm{LiNbO}_{3}$ crystal (Sirah DFM-2400-T) using about $280 \mathrm{~mJ}$ of the $1064 \mathrm{~nm}$ laser light. This generates up to $30 \mathrm{~mJ} 8 \mathrm{~ns}$ pulses of $1803.75 \mathrm{~nm}$ light with a bandwidth of less than $0.001 \mathrm{~cm}^{-1}$. 


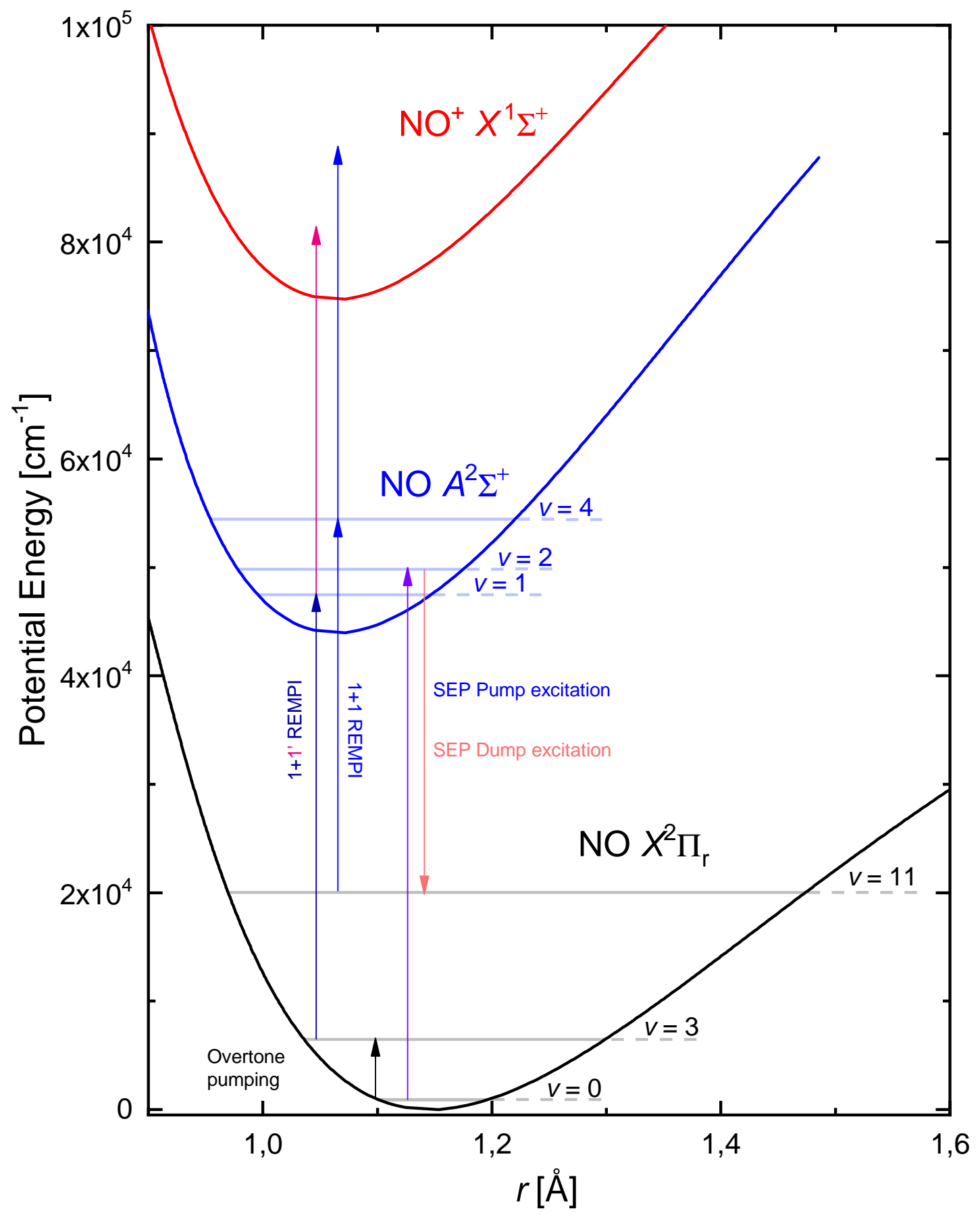

Figure 3.9: Calculated potential energy curves of the electronic states $X^{2} \Pi_{r}$ and $A^{2} \Sigma^{+}$of $\mathrm{NO}$ and $X^{1} \Sigma^{+}$of $\mathrm{NO}^{+}$in vacuum. The relevant vibrational states are added to the potential energy curves and marked with their quantum number $v$. For this work relevant transitions for preparation and detection of NO are indicated by arrows of different colors with matching labels. 


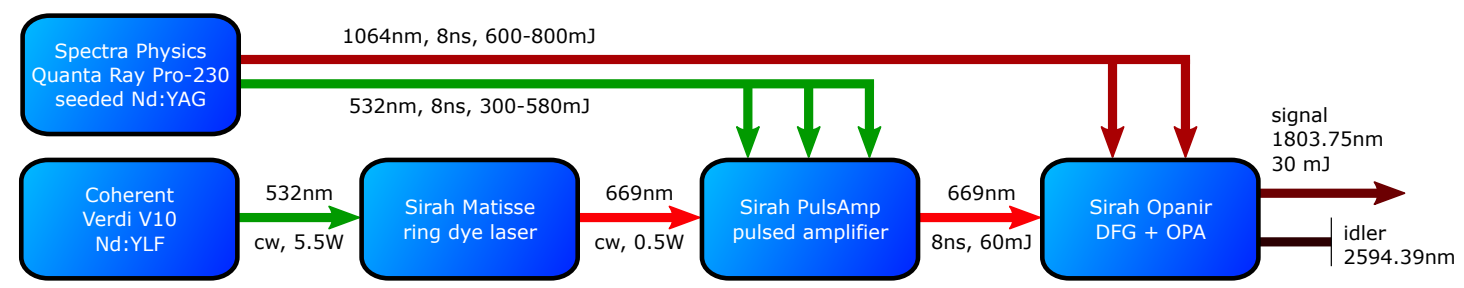

Figure 3.10: Schematic of the laser system used to generate $1803.75 \mathrm{~nm}$ laser light to excite the $\mathrm{X}^{2} \Pi(v=3) \leftarrow \mathrm{X}^{2} \Pi(v=0)$ transition in NO.

\subsubsection{1+1' REMPI of NO in $\mathrm{X}^{2} \Pi(v=3)$}

Ionization of $\mathrm{NO} \mathrm{X}^{2} \Pi(v=3)$ is done in a $1+1^{\prime}$ REMPI scheme. The molecules are initially excited via the $\gamma(1,3), \gamma(0,2)$ or $\gamma(0,1)$ transition and then ionized by a second photon. The first step occurs depending on the vibrational and rotational excitation of the NO molecule between $248.2 \mathrm{~nm}$ and $234.0 \mathrm{~nm}$. The second transition to the ionized state is done with $296.5 \mathrm{~nm}$. Therefore, this setup works with a 1+1' REMPI scheme. The laser light for the first transition is generated by a dye laser (Sirah, Cobra Stretch, CSTR-LG-24) with a dye mixture of Coumarin $102\left(0.3 \mathrm{~g} \mathrm{~L}^{-1}\right)$ and Coumarin 307 $\left(0.1 \mathrm{~g} \mathrm{~L}^{-1}\right)$ in ethanol. The output is then frequency-doubled in a $\beta-\mathrm{BaB}_{2} \mathrm{O}_{4}(\mathrm{BBO})$ crystal (Sirah, SHG-215). It is pumped with the SHG $(532 \mathrm{~nm}$ ) of a nanosecond pulsed Nd:YAG laser (Continuum, Surelight SL-III-Ex). Note that it is also possible to accomplish ionization in a $1+1$ REMPI with two photons with a wavelength of $248.2 \mathrm{~nm}$ to $234.0 \mathrm{~nm}$. A disadvantage of $1+1$ REMPI is that the ionization transition is competing with stimulated emission back to the initial state. 1+1' REMPI has therefore a much higher ionization probability which allows lower voltages on the MCP detector and in consequence reduces the noise in the signal. Also, the 1' pulse does not broaden the spectral lines because it only excite the ionization and not the transition to the A state. The light for the ionization is generated by the SHG (BBO, Sirah, SHG-250-T) of a second dye laser (Sirah, Cobra Stretch, CSTR-LG-18) with Rhodamin B $\left(0.2 \mathrm{gL}^{-1}\right)$ in ethanol as laser dye. The dye laser is pumped by a nanosecond pulsed Nd:YAG laser (Continuum, Powerlight, PL7010).

\subsubsection{Preparation of $\mathrm{NO}$ in $\mathrm{X}^{2} \Pi(v=11)$}

For the preparation of $\mathrm{NO} \mathrm{X}^{2} \Pi(v=11, J=0.5)$ a stimulated emission pumping (SEP) scheme was used since the direct selective excitation to $v=11$ would require unrealistic high light intensities due to the low excitation probability. In the used SEP scheme NO $\mathrm{X}^{2} \Pi(v=0, J=0.5)$ was first pumped via the $\mathrm{Q}_{11}$ branch transition to the $\mathrm{A}^{2} \Sigma^{+}(v=$ 


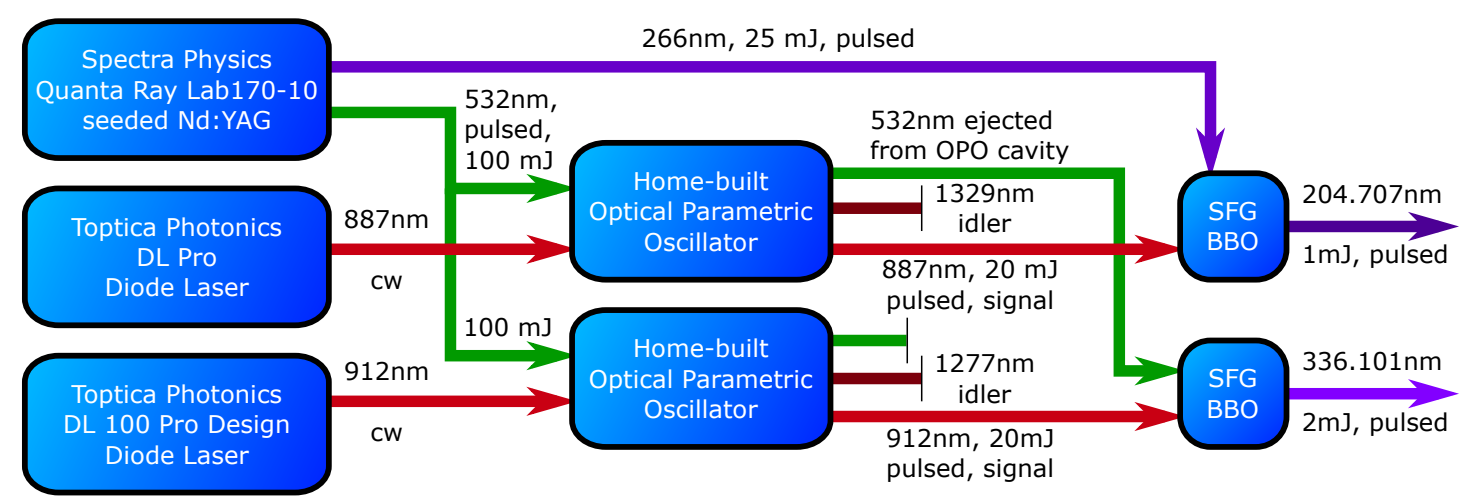

Figure 3.11: Schematic of the laser system used to generate $1803.75 \mathrm{~nm}$ laser light to excite the $\mathrm{X}^{2} \Pi(v=3) \leftarrow \mathrm{X}^{2} \Pi(v=0)$ transition in NO.

$2, J=0.5)$ state using $204.707 \mathrm{~nm}$ laser light. The molecules are then, after a delay of $3 \mathrm{~ns}$, dumped via the $\mathrm{Q}_{11}$ branch transition to the $\mathrm{X}^{2} \Pi(v=11, J=0.5)$ state using $336.101 \mathrm{~nm}$ laser light. The transitions are in the following called accordingly Pump and Dump transition.

The laser light for the Pump transition is generated by sum frequency generation (SFG) of the infrared output of a home-built optical parametric oscillator with the Fourth Harmonic Generation (FHG) of a Nd:YAG laser. The laser light for the Dump transition is generated by AFG of the infrared output of a second home-built optical parametric oscillator with the SHG of the same Nd:YAG laser. The schematic laser setup is shown in Figure 3.11. A detailed description of the used system can be found in the $\mathrm{PhD}$ thesis of Nils Bartels [43]. The OPOs are simultaneously pumped by the SHG of a injection seeded, pulsed Nd:YAG laser (Spectra Physics Quanta-Ray Lab170-10) with a total output of $200 \mathrm{~mJ}$. Each OPO is seeded by an individual $\mathrm{cw}$ external cavity diode laser (ECDL) with a wavelength of $887 \mathrm{~nm}$ for the Pump setup (Toptica Photonics, DL Pro) and $912 \mathrm{~nm}$ for the Dump setup (Toptica Photonics, DL 100 Pro Design). The signal output of the Pump OPO is used with the $266 \mathrm{~nm}$ FHG of the Nd:YAG laser for $\mathrm{SFG}$ in a $\beta-\mathrm{BaB}_{2} \mathrm{O}_{4}(\mathrm{BBO})$ crystal (Altechna Co. Ltd, $\Theta=58.3^{\circ}$ ) to generate light with a wavelength of $204.707 \mathrm{~nm}$. The signal output of the Dump OPO is used with the $532 \mathrm{~nm}$ (SHG) of the Nd:YAG laser for SFG in a second BBO crystal (Castech, $\left.\Theta=23.6^{\circ}\right)$ to generate light with a wavelength of $336.101 \mathrm{~nm}$. 


\subsubsection{1+1 REMPI of NO in $\mathrm{X}^{2} \Pi(v=11)$}

NO has several transitions from states between $\mathrm{X}^{2} \Pi(v=5)$ and $\mathrm{X}^{2} \Pi(v=13)$ in the frequency doubled laser dye range of Rhodamin B. The laser light is generated by a dye laser (Sirah, Cobra Stretch, CSTR-LG-18) with Rhodamin B laser dye $\left(0.20 \mathrm{~g} \mathrm{~L}^{-1}\right)$. The dye laser is pumped by a nanosecond pulsed Nd:YAG laser (Continuum, Powerlight, PL7010).

\subsection{Molecular Beam Apparatus}

Vibrationally excited $\mathrm{NO}$ was scattered from $\mathrm{VO}_{2}$ in the ultra high vacuum chambers of two different experimental setups. One is used for scattering of NO in low vibrational states, while the other is used for scattering highly vibrationally excited NO. The following section will give a brief description of both.

\subsubsection{Molecular Beam Apparatus for Surface Scattering of $\mathrm{NO}^{2} \Pi(v=3)$}

The experimental setup used for surface scattering of $\mathrm{NO} \mathrm{X}^{2} \Pi(v=3)$ has been described in detail previously [68]. This section will give a short description of specific settings and geometries used for this work and changes done to the setup described in Reference [68.

Figure 3.12 shows schematically the surface scattering setup. It consists of 4 chambers which are labeled as source, differential I, differential II and surface chamber with respective base pressures of about $10^{-8}, 10^{-8}, 10^{-9}$ and $10^{-10}$ mbar which rise by about one order of magnitude with the molecular beam running. The molecular beam is generated through supersonic jet expansion of $1 \%$ NO seeded in $\mathrm{H}_{2}$ with 3 bar stagnation pressure from a piezoelectric nozzle ( $1 \mathrm{~mm}$ orifice diameter, $10 \mathrm{~Hz}$ repetition rate). The piezoelectric crystal (PI, P288.23) is biased with a $70 \mu$ s pulse at $460 \mathrm{~V}$ to open the nozzle. The molecular beam then passes a skimmer with $1.5 \mathrm{~mm}$ diameter opening and the two differential pumped chambers with $2 \mathrm{~mm}$ openings before it enters the surface chamber. The source chamber is pumped by a turbomolecular pump (TMP) (Edwards, STP-A2203C) with a pumping speed of $3000 \mathrm{~L} \mathrm{~s}^{-1}$. The differential chambers are pumped by two TMPs with pumping speeds of $400 \mathrm{~L} \mathrm{~s}^{-1}$ (Seiko Seiki, STP-400) and $120 \mathrm{~L} \mathrm{~s}^{-1}$ (Osaka Vacuum Ltd., TF160CA). The surface chamber is pumped by a TMP (Leybold, NT360) with $350 \mathrm{~L} \mathrm{~s}^{-1}$ pumping speed which is backed by a second TMP (Pfeiffer-Balzers, TPU062) with a pumping speed of $70 \mathrm{~L} \mathrm{~s}^{-1}$.

The surface chamber is split into two interconnected parts where the surface scattering experiments are performed in the lower part and the surface preparation is performed in 


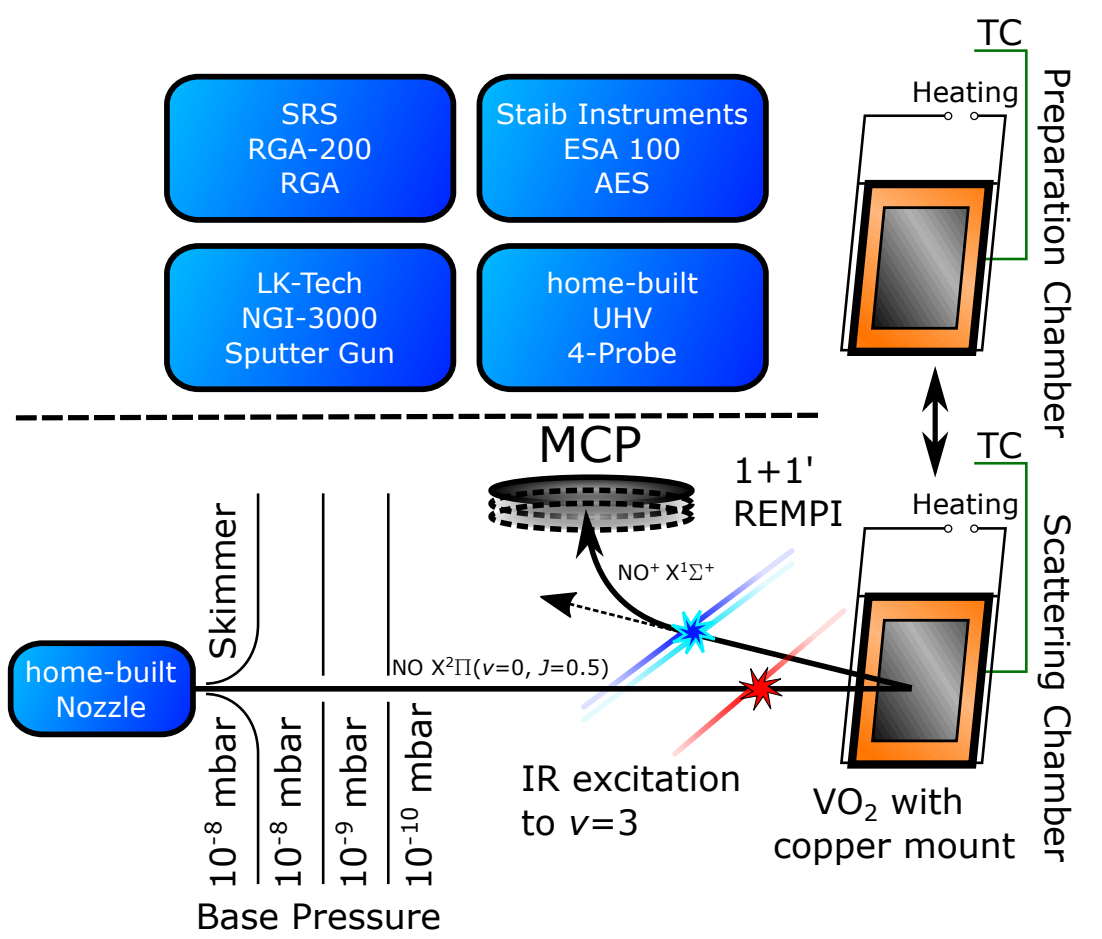

Figure 3.12: Experimental setup for the surface scattering of $\mathrm{NO} \mathrm{X}^{2} \Pi(v=3, J=0.5)$ from $\mathrm{VO}_{2}$ films. The molecular beam of $\mathrm{NO}$ seeded in $\mathrm{H}_{2}$ is generated in a piezoelectric nozzle and passes through three differentially pumped chambers before it reaches the scattering chamber with the surface. Before the NO reaches the surface it is excited through overtone pumping to $v=3$. After scattering from the $\mathrm{VO}_{2}$ surface it is ionized in a $1+1$ ' REMPI scheme and the ions are collected by a micro-channel plate (MCP) detector. The surface is heated by resistive heating of the surface mount. The sample mount can be moved to the preparation chamber above the scattering chamber where various device for surface preparation and characterization are located.

the upper part. The upper preparation chamber contains an Auger Electron Spectrometer (AES) (Staib Instruments, ESA 100) for characterization of the surface's elemental composition, a residual gas analyzer (RGA) (Stanford Research Systems, RGA-200) for characterization of the chamber atmosphere's chemical composition, an ion sputter gun (LK-Tech, NGI-3000) for surface preparation and a home-built 4-Probe to measure the surface resistance of the sample. The setup uses resistive heating of tantalum wires connected to the sample to heat it. The temperature is measured with a K-type thermocouple (Keithley, 2000 Multimeter) with manual cold junction compensation (CJC). Since the $\mathrm{VO}_{2}$ films are either deposited onto brittle r-cut sapphire or $\mathrm{Si} / \mathrm{SiO}_{2}$ substrates it is not possible to mount them directly to the heating wires. Therefore, the sample is mounted to a solid oxygen free high conducting (OFHC) copper mount to which both 
the heating wires and the thermocouple are attached. The surface can be moved along four axes $(x, y, z, \phi)$ by a UHV manipulator (Vacuum Generator Fisons Instruments, Omniax 800) to position it for characterization, preparation or surface scattering. The scattering chamber possesses two $\mathrm{CaF}_{2}$ windows through which the laser beams, used for excitation and resonance enhanced multi photon ionization(REMPI), are passed through. For REMPI spectroscopy a micro channel plate (MCP) detector is placed above the molecular beam path.

\subsubsection{Molecular Beam Apparatus for Surface Scattering of NO $\mathrm{X}^{2} \Pi(v=11)$}

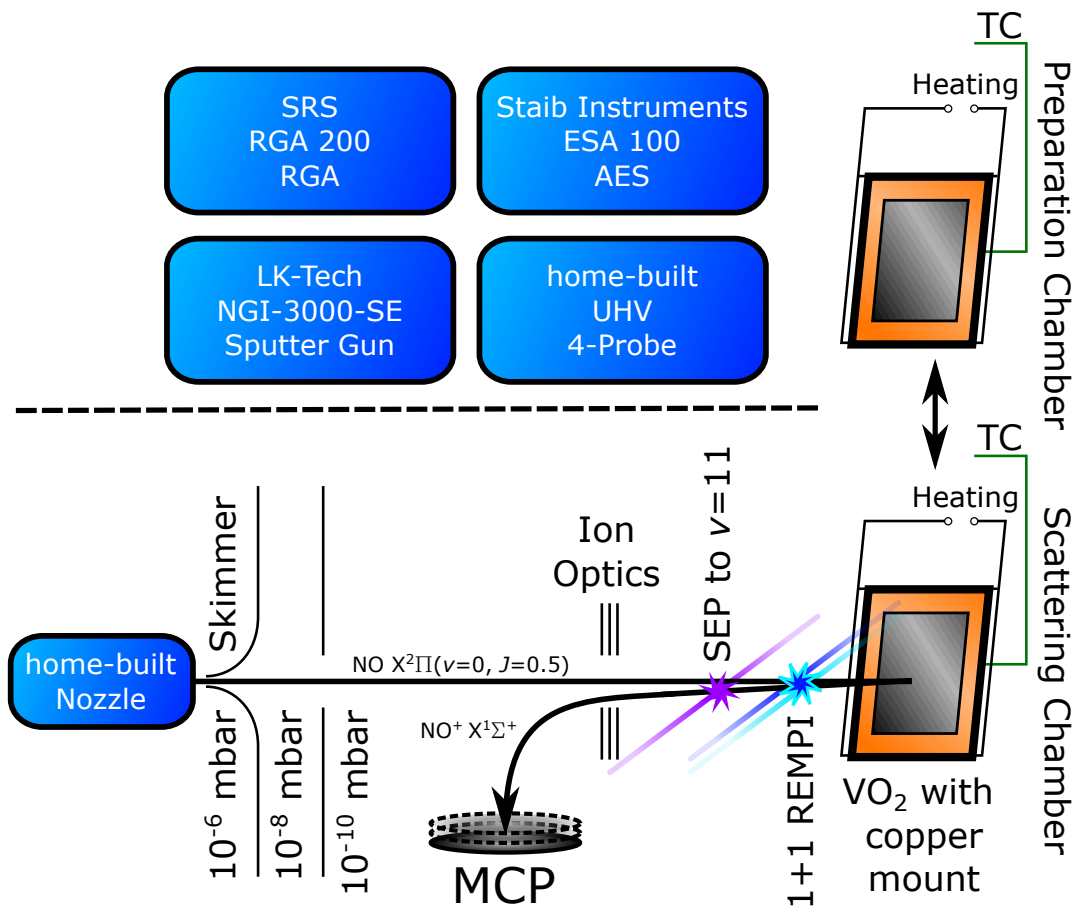

Figure 3.13: Experimental setup for the surface scattering of $\mathrm{NO} \mathrm{X}^{2} \Pi(v=11, J=0.5)$ from $\mathrm{VO}_{2}$ films. The molecular beam of $\mathrm{NO}$ seeded in $\mathrm{H}_{2}$ is generated in a home-built Evan-Lavie type nozzle and passes through three differentially pumped chambers before it reaches the scattering chamber with the surface. Before the NO reaches the surface it is excited through stimulated emission pumping to $v=11$. After scattering from the $\mathrm{VO}_{2}$ surface it is ionized in a $1+1$ REMPI scheme and the ions are collected by a micro-channel plate (MCP) detector. The surface is heated by resistive heating of the surface mount. The sample mount can be moved to the preparation chamber above the scattering chamber where various device for surface preparation and characterization are located. 
The experimental setup used for surface scattering of $\mathrm{NO} \mathrm{X}^{2} \Pi(v=11, J=0.5)$ has been described in detail previously [43]. This section will give a short description of the specific setting used for this work.

Figure 3.13 shows the schematic experimental setup. It consists of three chambers labeled as source, differential and surface chamber in the following. The base pressure of these chambers are respectively $10^{-6}, 10^{-8}$ and $10^{-10}$ mbar which all rise by about one order of magnitude when the molecular beam is running. The source chamber holds a home-built Evan-Lavie type nozzle [69, designed and constructed by Prof. Dr. Schwarzer. It generates a pulsed molecular beam with $10 \mathrm{~Hz}$ repetition rate. The nozzle is run with mixtures of $1,2,5$ and $10 \% \mathrm{NO}$ seeded in $\mathrm{H}_{2}$ with a stagnation pressure of 6 bar. The source chamber is pumped with $400 \mathrm{~L} \mathrm{~s}^{-1}$ by a TMP (Osaka Vacuum Ltd., TS443).

The differential chamber is connected to the source chamber by a $2 \mathrm{~mm}$ electro-formed skimmer (Beam Dynamics Inc., NI Model 2). It is pumped by a TMP with $360 \mathrm{~L} \mathrm{~s}^{-1}$ pumping speed (Leybold, Turbovac 360CSV). The molecular beam leaves the differential chamber to the surface chamber through a $2 \mathrm{~mm}$ aperture.

The surface chamber is, similar to the setup described above, split into two parts labeled in the following as preparation and scattering chamber. The preparation chamber contains instruments for surface preparation and characterization such as an Auger Electron Spectroscope (AES) (Staib Instruments, ESA 100) for characterization of the surface's elemental composition, a residual gas analyzer (RGA) (Stanford Research Systems, RGA-200) for characterization of the chamber atmosphere's chemical composition, an ion sputter gun (LK-Tech, NGI-3000-SE) for surface preparation and a home-built 4Probe to measure the resistance of the sample. The surface is mounted to a solid OFHC copper frame which itself is held by either tungsten or tantalum wires. Resistive heating of the mounting wires is used to heat the $\mathrm{VO}_{2}$ surface while a K-type thermocouple fixed to the OFHC copper frame is used to monitor the temperature. The thermocouple voltage is read by a NI-9213 voltmeter by National Instruments. The sample can be moved along four axes $(x, y, z, \phi)$ by a UHV manipulator (U.H.V Instruments, model number: 30007651110) to position it for measurements and transfer it between preparation and scattering chamber. Laser beams used for preparation of the NO molecules enter the surface through two UV-fused silica windows facing each other. 


\subsection{Experimental Procedures}

\subsubsection{Timings and MCP detector}

Surface Scattering of NO $X^{2} \Pi(v=3)$

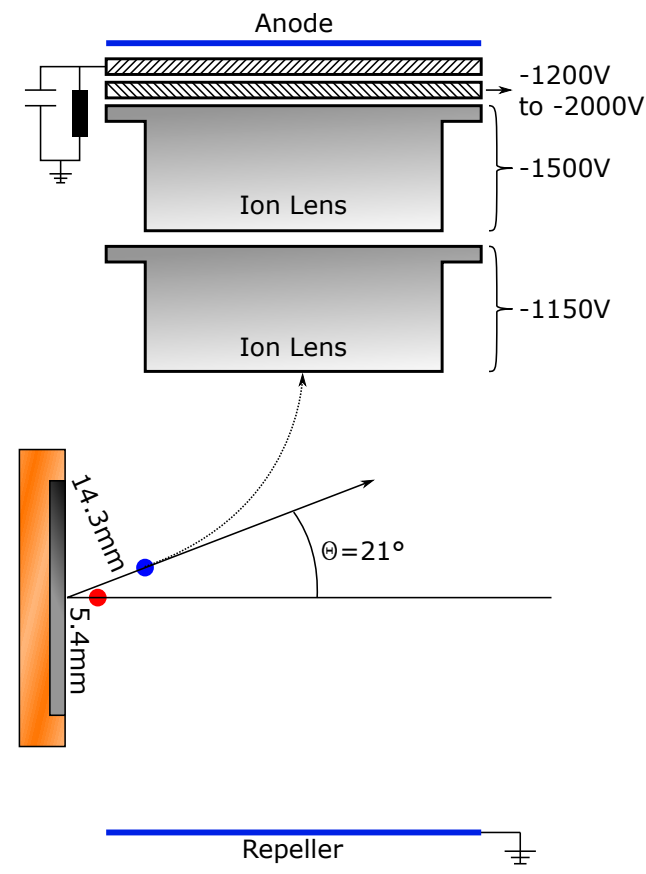

Figure 3.14: Ion detector setup with molecular beam and laser positions from a typical NO $\mathrm{X}^{2} \Pi(v=3)$ time-of-flight experiment.

The nozzle is held open for $40 \mu \mathrm{s}$ with at a repetition rate of $10 \mathrm{~Hz}$. After $184 \mathrm{\mu s}$ the pulsed Nd:YAG pump laser of the IR laser system fires. The timing to fire the pump laser for the initial transition of the 1+1' REMPI scheme is varied from $180 \mu \mathrm{s}$ to $290 \mu \mathrm{s}$ after the nozzle opens. The laser to ionize the excited molecules fires $10 \mu$ s after the pump laser.

The ions are detected with a double MCP detecter in chevron configuration with an ion lens consisting of two cylindrical elements. The ion lens is charged with $-1150 \mathrm{~V}$ and $-1500 \mathrm{~V}$ to attract and guide ions from the ionization region to the MCP plates which are charged with $-1200 \mathrm{~V}$ to $-2000 \mathrm{~V}$ depending on the desired amplification gain. Furthermore, the charge of MCP is only applied for $6 \mu$ s set to switch on shortly after the arrival of the REMPI laser pulse in the chamber. Otherwise it is reduced to $1200 \mathrm{~V}$ to avoid the saturation of the detector by stray UV light from the crossing laser beam pulses. A grounded repeller plate is located on the opposite side of the molecular beam path as shown in Figure 3.14. The amplified signal is transferred to an anode 
behind the MCPs and is displayed by a digital oscilloscope (LeCroy, WaveSurfer 104 WXs-B). The data from the oscilloscope is then processed in a home-built LabVIEW environment (National Instruments, LabVIEW 2010).

\section{Surface Scattering of $\mathrm{NO} \mathrm{X}^{2} \Pi(v=11)$}

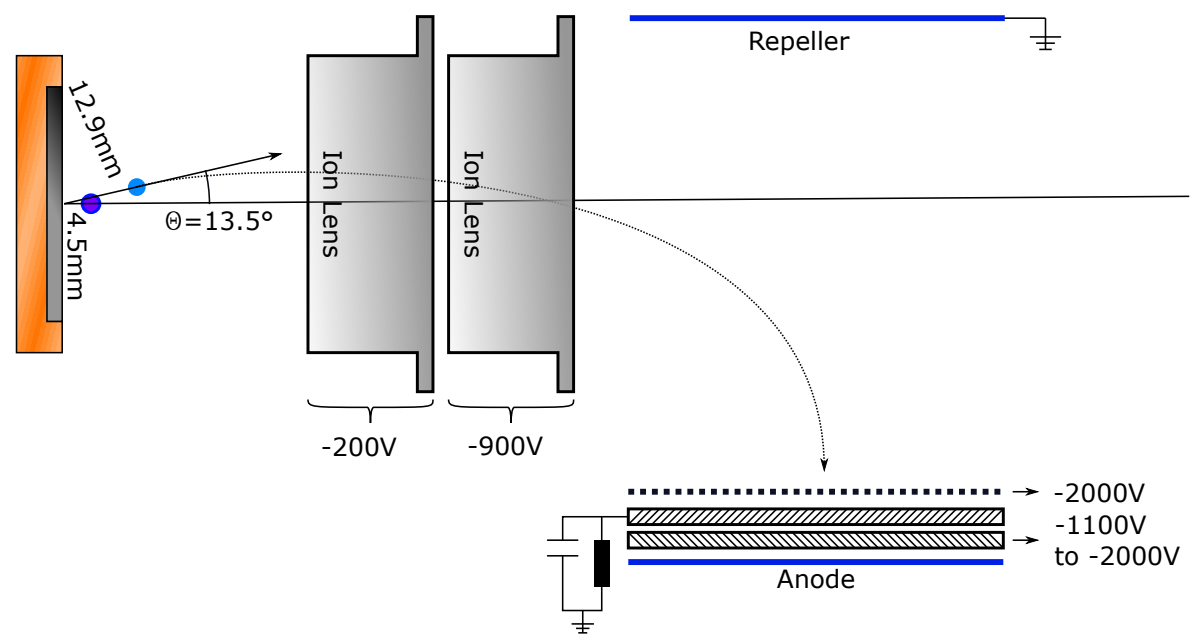

Figure 3.15: Ion detector setup with molecular beam and laser positions from a typical NO $\mathrm{X}^{2} \Pi(v=11)$ time-of-flight experiment.

The nozzle opens every $100 \mathrm{~ms}$. $293 \mu$ s after the nozzle opening the pump laser of the two OPO systems fires and generates both the Pump and the Dump light pulse to prepare NO $\mathrm{X}^{2} \Pi(v=11)$. The beam pathway is designed so that the Dump pulse arrives 2 ns after the Pump pulse. The Pump laser of the REMPI laser setup can be set up to fire $1 \mu$ s to $50 \mu$ s after the OPO setup. It arrives $1.13 \mu$ s earlier than the Pump pulse if the timing of OPO and REMPI pump laser are set to the same value.

The detector consists of a chevron configured MCP (tectra, $25 \mathrm{~mm}$ diameter) with a grid to attract the ions onto the MCP placed in front of it. The MCP is charged with $-1100 \mathrm{~V}$ to $-2000 \mathrm{~V}$ depending on the desired amplification gain. The MCP charge is also only applied between $239.5 \mu$ s to $462.5 \mu$ s after the nozzle opens to avoid MCP saturation through stray UV light from the crossing beam pulses. The amplified signal from the MCP is detected by an anode and displayed on a digital oscillator (Lecroy, Waverunner LT344). The data is processed by a home-build LabVIEW environment (National Instruments, LabVIEW 2009). Along the molecular beam path an ion lens is placed consisting of two elements charged with $-200 \mathrm{~V}$ and $-900 \mathrm{~V}$ to focus and guide the $\mathrm{NO}^{+}$ions to the detector. 


\subsubsection{Time-of-Flight Analysis}

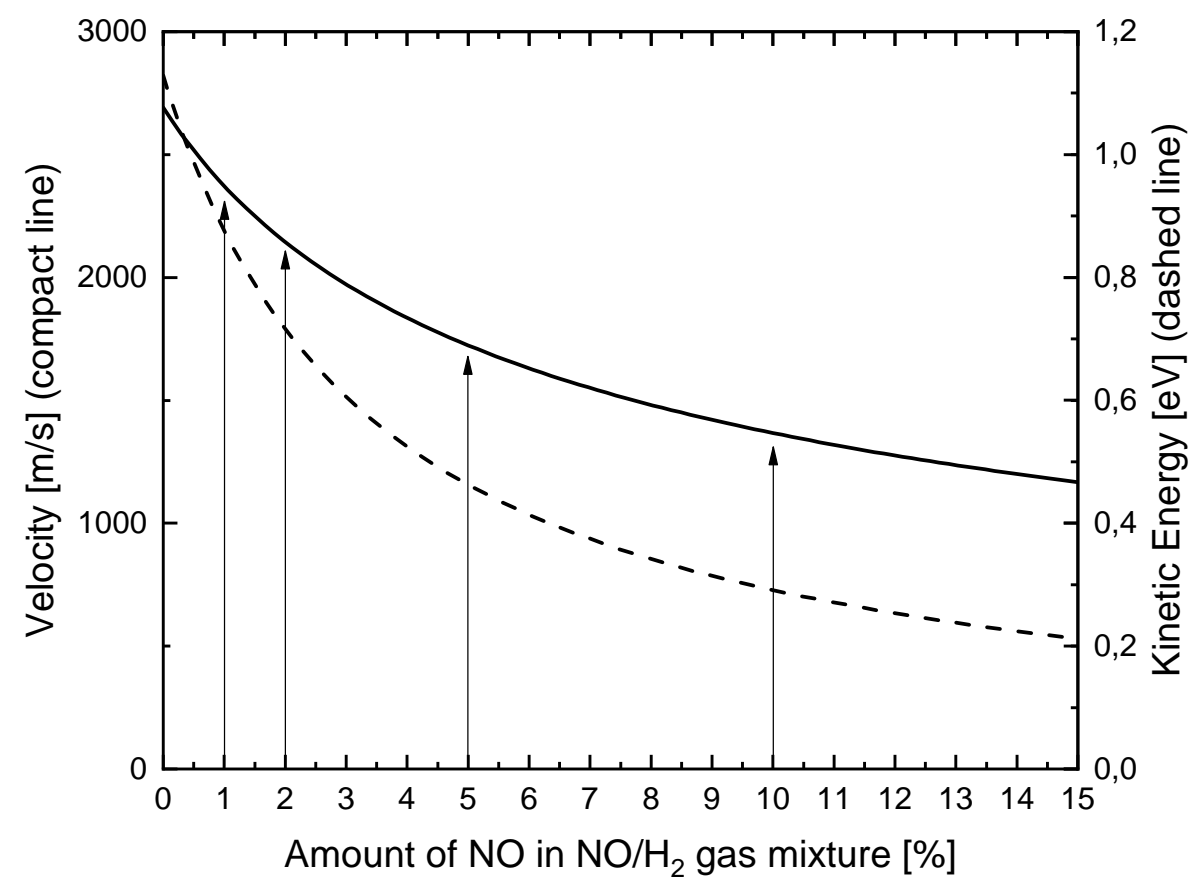

Figure 3.16: Velocity and translational energy of different $\mathrm{NO} / \mathrm{H}_{2}$ gas mixtures. Arrows indicate gas mixtures used in this work.

Time-of-flight (TOF) distributions are measured by first determining the velocity of the incident beam which is dependent on the gas mixture ratio of the seed gas, in most cases hydrogen, and NO. Since the molecular beams are all generated in a supersonic jet expansion the velocity of the molecular beam $v_{\text {inc }}$ can be estimated by the following equation 70

$$
v_{\text {inc }}=\sqrt{\frac{2}{\bar{m}} \overline{c_{\mathrm{p}}} T_{0}} .
$$

$\bar{m}$ is the average mass of the expanded molecules and $\overline{c_{\mathrm{p}}}$ is the average heat capacity of the gas mixture. Since both, hydrogen and NO, are both diatomic molecular gases $\overline{c_{\mathrm{p}}}$ should be approximately independent of the gas mixture. The lighter the seed gas and the higher percentage of the lighter gas the lower $\bar{m}$ becomes. In consequence $v_{\text {inc }}$ becomes faster. $T_{0}$ is the gas temperature upon expansion which equals approximately the nozzle temperature which is left at room temperature $T_{\text {nozzle }} \approx 300 \mathrm{~K}$ in this work. Figure 3.16 shows both the estimated velocity and the translational energy of a molecular beam from supersonic jet expansion of a $\mathrm{NO} / \mathrm{H}_{2}$ gas mixture. The gas mixtures used in this work are marked with arrows. 
To measure the velocity of the incident molecular beam the surface is moved out of the molecular beam path to avoid scattering and enable a longer beam path. The molecular beam of $\mathrm{NO} \mathrm{X}^{2} \Pi(v=0)$ is then tagged with the IR or Pump beam to the $v=3$ or $v=11$ state. Simultaneously the REMPI laser is set to cross the molecular beam at a known distance $l$ downstream from the tagging laser beam. This was usually around $10 \mathrm{~mm}$ to $20 \mathrm{~mm}$. By scanning the REMPI timing described in section 3.5.1 a temporal distribution of the beam pulse of tagged molecules is measured. The difference between the tagging laser timing and the REMPI laser timing with some compensation for electronic delays and laser beam flight time is equal to the flight time of the tagged molecules. The time-of-flight distribution can be described by a three dimensional velocity distribution

$$
f_{\text {flux }}(v)=v^{3} \cdot \exp \left(-\left(\frac{v-v_{0}}{\alpha}\right)^{2}\right) .
$$

$v$ is the velocity of the beam and $v_{0, \text { inc }}$ the mean velocity of the distribution. $\alpha_{\text {inc }}$ is a parameter that characterizes the width of the exponential term or the spread of the described beam pulse [70].

Since the velocity distribution cannot be directly measured it is necessary to measure the time-dependent distribution of the molecules in the molecular beam pulse tagged by the Pump laser and fit it to the parameters of Equation 3.3 .

The intensity of a laser excitation is proportional to the number of particles in the volume of the laser beam pulse. This means that the measured distribution is a density distribution in contrast to the Equation 3.3 which is a distribution of flux. The flux distribution is converted to density by dividing it through the velocity $v$ once for every laser excitation, in this case two times (Pump and REMPI excitation):

$$
f_{\text {density }}(v)=\frac{f_{\text {flux }}(v)}{v^{2}}=v \cdot \exp \left(-\left(\frac{v-v_{0}}{\alpha}\right)^{2}\right) .
$$

To transform from the velocity to the time domain the function has furthermore to be multiplied by the Jacobian:

$$
f_{\mathrm{ds}}(t)=\frac{f_{\text {flux }}(v)}{v^{2}} \frac{\mathrm{d} v}{\mathrm{~d} t}=A \cdot\left(\frac{t}{l}\right)^{2} \cdot\left(\frac{l}{t^{2}}\right) \cdot\left(\frac{l}{t}\right)^{3} \cdot \exp \left(-\left(\frac{l / t-v_{0}}{\alpha}\right)^{2}\right) .
$$

For the case of a TOF distribution that does not originate from a scattering event but from a desorption process that is not influenced by the initial conditions of the incoming molecular beam the Equation 3.5 has to be modified. First the velocity of the molecules $v$ in Equation 3.4 is not influenced by the incident velocity $v_{0}$. Therefore, this parameter is dropped. Also the molecular beam volume only interacts with one laser during the 
ionization. Therefore, $f_{\text {flux }}(v)$ is only divided once by $v$ to transform it to $f_{\text {density }}(v)$. The result is the equation

$$
f_{\mathrm{td}}(t)=\frac{f_{\text {flux }}(v)}{v} \frac{\mathrm{d} v}{\mathrm{~d} t}=A \cdot\left(\frac{t}{l}\right) \cdot\left(\frac{l}{t^{2}}\right) \cdot\left(\frac{l}{t}\right)^{3} \cdot \exp \left(-\left(\frac{l / t}{\alpha}\right)^{2}\right)
$$

The Equations 3.5 and 3.6 are used to fit the measured time-dependent distribution and extract the parameters $v_{0}$ and $\alpha$ for Equation 3.3 . The mean velocity $\langle v\rangle$ is then calculated with the equation

$$
\langle v\rangle=\int_{0}^{\infty} v f_{\text {flux }}(v) \mathrm{d} v
$$

With the mean velocity $\langle v\rangle$ the translational Energy $E_{0}$ of the mean velocity is calculated:

$$
E_{0}=\frac{1}{2} m(\mathrm{NO})\langle v\rangle^{2} .
$$

With $E_{0}$, the beam spread parameter $\alpha$ and the mass of the NO molecule $m(\mathrm{NO})$ the energy distribution is obtained using Equation 3.9 .

$$
f(E)=\frac{2 E}{m(\mathrm{NO})^{2}} \cdot \exp \left(-\left(\frac{\sqrt{\frac{2 E}{m(\mathrm{NO})}}-\sqrt{\frac{2 E_{0}}{m(\mathrm{NO})}}}{\alpha}\right)^{2}\right)
$$

Analogous to the mean velocity $\langle v\rangle$ described above the mean translational energy of scattered NO can be obtained

$$
\langle E\rangle_{\operatorname{trans}}=\int_{0}^{\infty} E f(E) \mathrm{d} E .
$$

The time-dependent TOF from Equation 3.3 used to fit the experimental data is dependent on the flight distance $l$ which is the main contributor to measurement inaccuracy in this experiment. It is determined by moving the surface trough the laser beams by adjusting the micrometer screw of the translation stage of the surface mount along the molecular beam path. Simultaneously, the transmitted laser power of the exiting beam is measured. The position of the laser beam is determined as the point where half of the laser power is block by the surface. Since the $\mathrm{VO}_{2}$ films are delicate and may be damaged by the intense preparation and detection laser beams they are usually substituted by a low power cw laser pointer in the same position as the actually used laser beam. From the sigmoid like intensity function of the micrometer screw position a flight distance error $\Delta l$ of either $0.3 \mathrm{~mm}$ or $0.5 \mathrm{~mm}$ was determined depending on the experimental setup. The function from Equation 3.5 is fit to the experimental data with 
the parameters $l, l+\Delta l$ and $l-\Delta l$ from which a mean, a minimum and a maximum velocity and translational energy are obtained. The half of the difference of the latter two is used as a the error of the former.

The translational energies gained from the TOF analysis are used to draw a Baule plot which shows the loss of translation energy of the molecules during the scattering event. Usually, the translational energies follow a linear progression if plotted against the rotational energy. By fitting them with a linear function the translational energy of scattered molecules in $J=0$ can be gained as the y-axis intercept. The translational energy adjusted for rotational excitation is then plotted against the corresponding translational energy of the incident molecular beam. In the hard sphere model ${ }^{71}$ scattered molecules follow a linear progression. The slope of this linear progression can be described by the following equation

$$
b=\frac{\left(m_{1}-m_{2}\right)^{2}}{\left(m_{1}+m_{2}\right)^{2}} .
$$

If the mass of the scattered molecules $m_{1}$ is known this method can be used to determine the effective mass $m_{2}$ of the surface the molecules are scattered from.

\subsubsection{REMPI Spectrum Analysis}
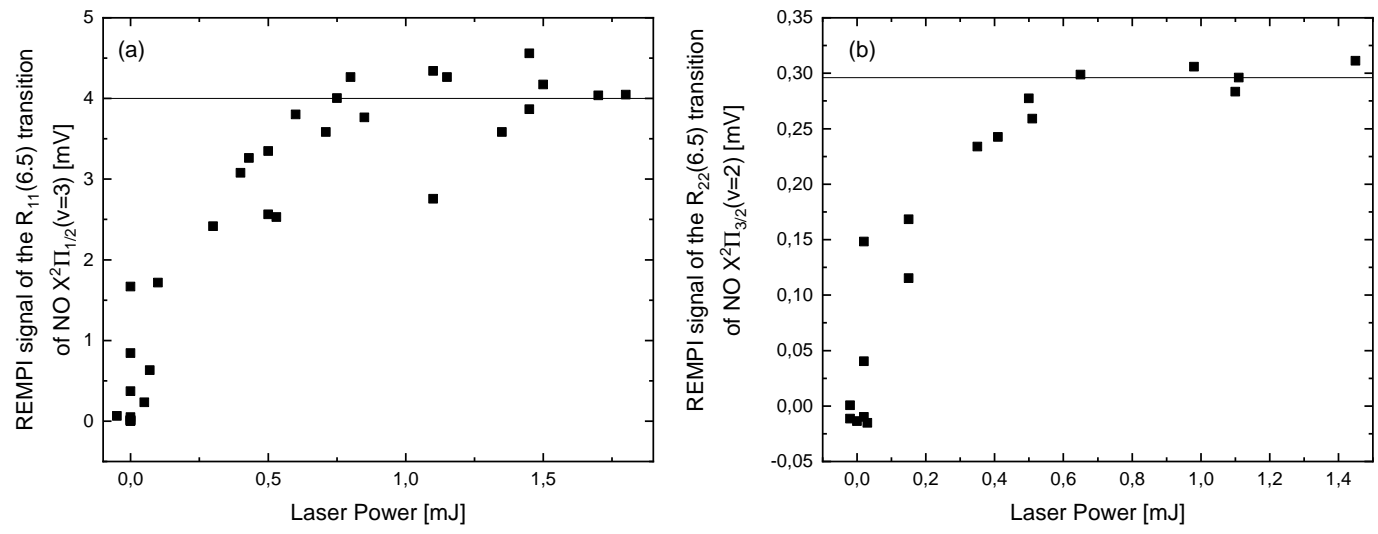

Figure 3.17: REMPI signal in dependence of the REMPI laser power for two transition lines of $\mathrm{NOX}^{2} \Pi_{\mathrm{r}}$.

REMPI spectra were performed at an arrival time where directly scattered molecules are expected. This is ensured by probing at an arrival time of $2 \mu$ s earlier than the maximum of the TOF distribution. During the measurement the power of the REMPI laser was measured after exiting the chamber to allow correction of the spectral intensity for laser power fluctuation. Figure 3.18 shows a visualization of the analysis process of REMPI spectra. 
Recording a REMPI spectrum of surface scattered NO X ${ }^{2} \Pi_{\mathrm{r}}(v=3)$ the laser power dependence is measured by observing the relative signal strength of the $\mathrm{R}_{11}(6.5)$ transition of $\mathrm{X}^{2} \Pi_{\mathrm{r}}(v=3)$ and the $\mathrm{R}_{22}(6.5)$ transition of $\mathrm{X}^{2} \Pi_{\mathrm{r}}(v=2)$ in dependence of the power of the REMPI beam. The result can be seen in Figure 3.17. They show that the signal reaches a saturation above a laser power of $0.6 \mathrm{~mJ}$ in both cases. In most cases laser powers around $1 \mathrm{~mJ}$ were used so the signal can be assumed to be saturated at all times. Therefore, no correction of the laser power was performed on these spectra. Furthermore, with overtone pumping the molecules are solely prepared in the desired state of $\mathrm{X}^{2} \Pi_{\mathrm{r}}(v=3)$ with the background consisting only of residual $\mathrm{X}^{2} \Pi_{\mathrm{r}}(v=0)$ and $\mathrm{X}^{2} \Pi_{\mathrm{r}}(v=1)$. During the experiments it could be seen that only a negligibly small amount of scattered NO relaxes to $\mathrm{X}^{2} \Pi_{\mathrm{r}}(v=1)$. In consequence, in all following experiments only transitions from $v=2$ and $v=3$ were measured.

Furthermore, in the experiments with $\mathrm{NO}(v=11)$ a number of other vibrational states are also populated through spontaneous emission from the excited $\mathrm{A}^{2} \Sigma^{+}$state. Background spectra with a blocked Dump laser are measured to subtract these populations. The spectra are analyzed for the vibrational and rotational population distribution by fitting a simulated spectrum to the experimental data. This method is described by Jacobs ${ }^{40]}$ and the method to construct simulated NO spectra from empirical parameters is based on the effective Hamiltonian by Brown $\underline{47}$. It is described in detail in Section 2.2 .3 .

The relative transition efficiencies of the individual vibrational bands are determined by measuring spectra of the incident molecular beam populated by Franck-CondonPumping from the $\mathrm{A}^{2} \Sigma^{+}(v=2)$ state. From this a raw vibrational distribution is gained which is influenced by the emission coefficients between the individual vibrational states of the ground electronic state and the excited $\operatorname{NOA}^{2} \Sigma^{+}(v=2)$ state and the individual REMPI efficiency of the $v$ states. The emission coefficients for each probed vibrational transition band from the $\mathrm{NOA}^{2} \Sigma^{+}(v=2)$ state are known from the literature ${ }^{72]}$. Therefore, the vibrational distribution of the molecular beam is known. By comparing each relative vibrational state population of the raw distribution with the actual vibrational distribution of the molecular beam gives the REMPI efficiency for each probed vibrational transition band. Using these REMPI efficiencies on later measured spectra allows to determine the vibrational distribution of the scattered NO molecules.

The simulated spectrum is fitted to the individual vibrational transition bands of the experimental REMPI spectrum by varying the respective rotational populations. This way the rotational population distribution of each probed vibrational transition band is 


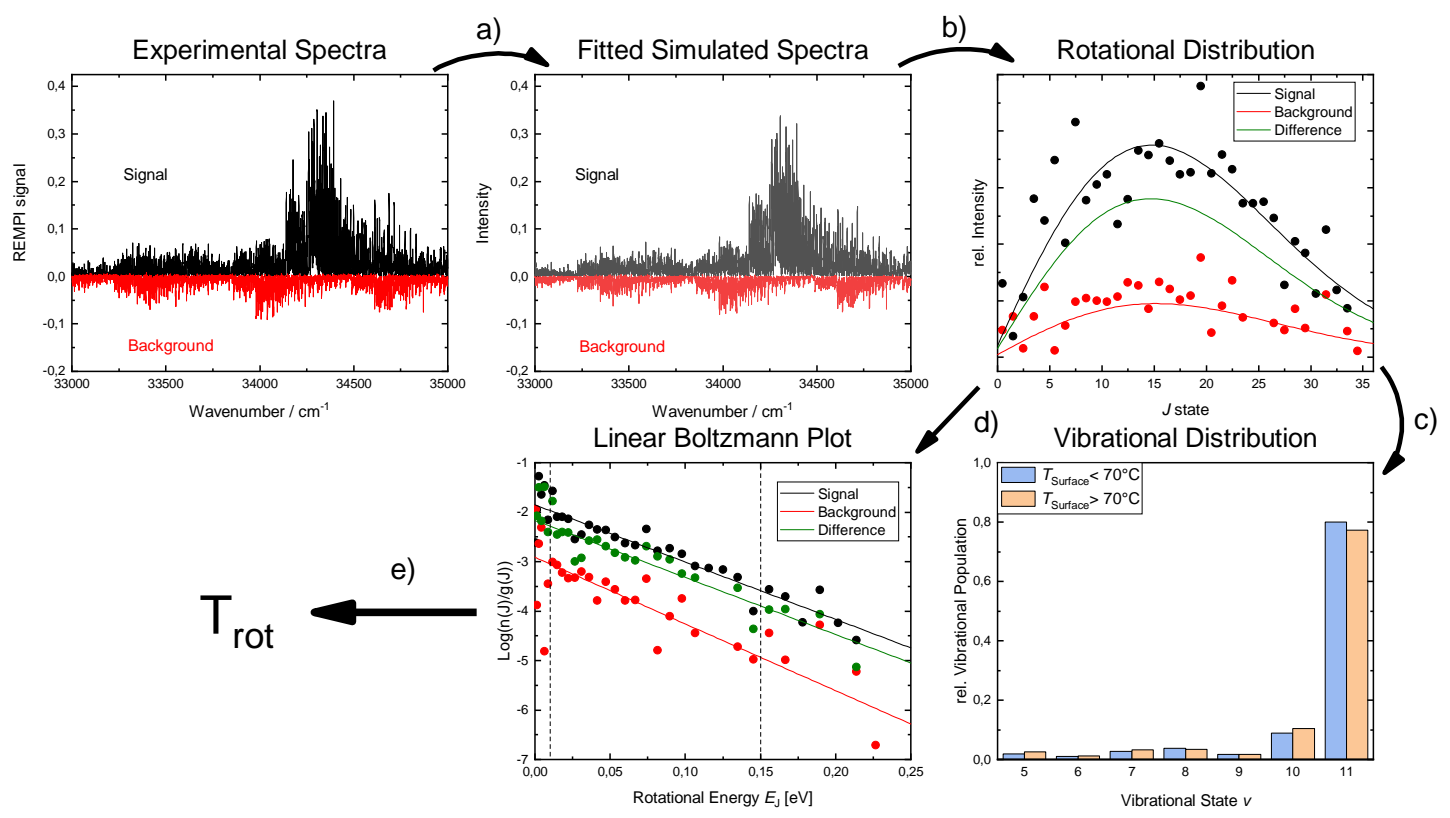

Figure 3.18: Process of REMPI spectrum analysis. First the experimental spectra (top left) are measured. This is done both with the Dump laser (signal) and without the Dump laser (background). In step a) the experimental REMPI spectra are fitted with simulated spectra (top center). In step b) the rotational populations of the observed transition bands are gained as fitting parameter. The rotational population distributions (top right) are fitted with a Boltzmann function (see Equation 3.12). The background population is reduced by a factor of 0.8. This is done because the Dump transition depopulates the $\operatorname{NOA}^{2} \Sigma^{+}(v=2)$ from which the background population is formed via spontaneous emission. The correction factor is gained from comparison between incident molecular beam spectra with and without Dump laser. In step c) the Boltzmann function of signal and background are subtracted from each other and resulting difference is integrated. This generates the vibrational population of each probed transition band (bottom right). In step d) the rotational distributions are plotted as a logarithmic plot or linear Boltzmann (bottom center). In step e) the rotational temperature is extracted from the slope of the linear plot. 
derived from the experimental REMPI spectra. The rotational population distribution is fitted with a Boltzmann distribution

$$
N(J)=N_{0} \cdot g(J) \cdot \exp \left(\frac{E(J)}{k_{\mathrm{B}} T_{\mathrm{rot}}}\right) .
$$

$N(J)$ is the population of the rotational state $J$ relative to the total rotational population $N_{0} . E(J)$ is the rotational energy, $g(J)=2 J+1$ is the degeneracy of the $J$ state and $k_{\mathrm{B}}$ the Boltzmann constant.

From the normalized rotational distributions the mean rotational energy $\langle E\rangle_{\text {rot }}$ is obtained. For this the following equation is used

$$
\langle E\rangle_{\mathrm{rot}}=\int_{0}^{J_{\max }} J \cdot \frac{N(J)}{N_{0}} \mathrm{~d} J .
$$

The values for each probed surface temperature are averaged and the error of $\langle E\rangle_{\text {rot }}$ is obtained from the standard deviation.

From Equation 3.12 the logarithmic Boltzmann function is obtained:

$$
\ln \frac{N(J)}{N_{0} \cdot g(J)}=\frac{E(J)}{k_{\mathrm{B}} T_{\mathrm{rot}}} .
$$

From this function the temperature of the distribution of rotational states $T_{\text {rot }}$ is extracted. The logarithmic Boltzmann function has the advantage that it is easy to see at which point, at high and low $J$, the noise of the distribution becomes significant. The quality of the fit is increased by neglecting these points.

The error of $T_{\text {rot }}$ is obtained by the "95\% Confindence Interval" option of the "LinearModelFit" function of Wolfram Mathematica 11.2. From here a maximum and minimum slope is obtained which is used to calculate the error interval of the rotational temperature.

It should be noted that while the description above uses the Boltzmann equation for the analysis of rotational population distributions rotationally excited molecules have no reason to show a thermal rotational population distribution. In consequence, on can not expect them to follow a Boltzmann distribution. The rotational temperature $T_{\text {rot }}$ is in the following used to see if the probed rotational population distributions are thermally equilibrated with the surface temperature. This would be an indicator for a trapping desorption mechanism during the scattering. If the rotational temperature $T_{\text {rot }}$ differs significantly from the surface temperature the molecules rotational excitation is generated from its initial translational energy. This is an indication for a short interaction time with the surface and an indicator for direct scattering. 


\subsubsection{Angular Distribution Procedure}

Angular distributions are used as an indicator to distinguish between the direct scattering and other non-direct channels when $\mathrm{NO}$ is scattered from the $\mathrm{VO}_{2}$ surface. When the translational energy of the incident molecules is retained after the collision the molecules scatter back with the same angle with respect to the surface normal. If the molecules desorp from the surface after they had time to equilibrate with the surface they loose all initial information. In consequence, they desorp along the surface normal and show $a \cos (\Theta)$ distribution. For scattered particles that retain their initial information the angular distribution should follow a $\cos ^{>1}(\Theta)$ distribution ${ }^{\text {[71. }}$. Also they should have an angular offset with respect to the surface normal depending on the incoming angle of the incident molecular beam.

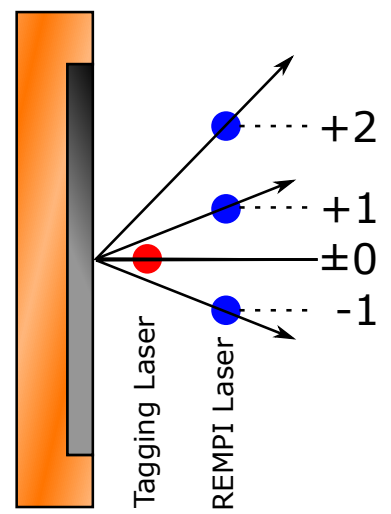

Figure 3.19: Schematic setup of the Angular Distribution setup.

For the measurement of angular distributions of NO scattered from a surface a setup similar to the one for TOF distribution measurements is used. A schematic of the setup is shown in Figure 3.19. The vertical position of the REMPI laser spot is moved parallel to the surface and ionizes scattered molecules at different angles. At each position a TOF distribution is measured. The integral of the TOF distribution is proportional to the beam intensity at this particular position. Since the relative positions between the surface and the laser spots are known the angles between these vectors can easily be calculated.

One has to keep in mind that since only the vertical position of the REMPI laser beam is moved, the flight path between the laser spot and the surface is longer for larger angles. Therefore, as the beam dilutes with increasing distance from the surface the measured intensity is usually lower than the actual intensity at larger angles. This error is in this work assumed to be negligible since the angular range is rather small. The angular distribution is only used as a qualitative indicator for direct scattering. 
Also the REMPI detection efficiency along the surface plane is not homogeneous due to the geometry of the ion lens and MCP geometry. This error is also neglected because it slightly distorts the distribution but should not be relevant for the qualitative distinction between desorption and direct scattering. 



\section{Scattering of NO from CVD prepared $\mathrm{VO}_{2}$ films}

This chapter describes the NO scattering experiments with CVD prepared $\mathrm{VO}_{2}$ films. Since in the past molecular scattering was conducted from atomically flat single crystal surface it is not clear if the direct scattering channel can be observed from sample with microscopically rough surfaces. It was assumed that the high amount of edges on the surface will result in a high sticking probability of incident NO. Therefore, this section will mainly analyze the properties of scattered NO from CVD prepared $\mathrm{VO}_{2}$ films with regard to their conservation of translational momentum and their thermal equilibration with surface degrees of freedom.

\subsection{Scattering of $N O(v=0)$ and $N O(v=11)$ from CVD1 and CVD2}

\subsubsection{Samples Characteristics of CVD1 and CVD2}

CVD1 and CVD2 were the first two samples produced by the CVD apparatus described in Section 3.2.1. It was never verified if these samples have a MIT. In contrast to later samples the films of $\mathrm{VO}_{2}$ are deposited in both cases on a $\mathrm{SiO}_{2}$ substrate. Images of them are shown in Figure 4.1 as an inset.

The surface of CVD1 appears to be reflective with large flakes peeling off while CVD2 has a mostly homogeneous matt surface with a grained structure. Due to the early stage of the project neither in Tel Aviv nor in Göttingen a 4-Probe device (see Section 3.1) was available. The resistance in dependence of temperature could only be measured several months after the scattering experiment were performed. The resistance-temperature curves are shown in Figure 4.1. CVD1 shows a high resistance of about $10^{5} \Omega$ which decreases with temperature as it is typical for a semi-conductor. CVD2 has a much lower resistance of about $10^{1} \Omega$ with much lower anti-proportional temperature dependence. This suggests that also has semi-conductor properties. Neither surface exhibits any MIT transition in the range between $30^{\circ} \mathrm{C}$ and $120^{\circ} \mathrm{C}$. CVD1 and CVD2 were not sputtered 


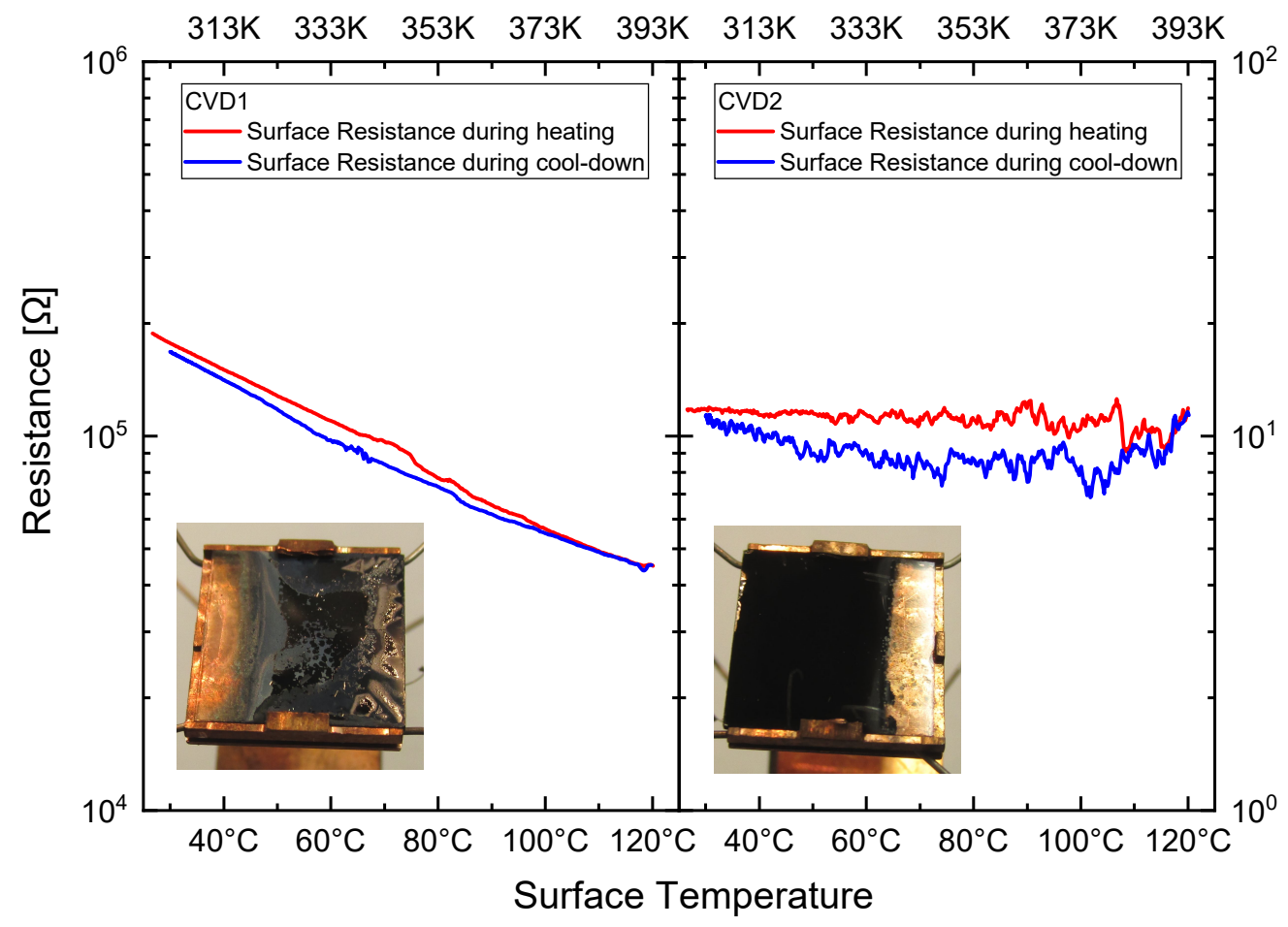

Figure 4.1: Resistance against temperature scan for the samples CVD1 and CVD2 measured under atmospheric conditions after surface scattering experiments. Insets show pictures of the samples taken before being built in into the UHV chamber.

with $\mathrm{Ar}^{+}$ions, as it is usually done in surface scattering experiments with metal singlecrystals. The samples were only repeatedly annealed to $400^{\circ} \mathrm{C}$ for up to $20 \mathrm{~min}$ to evaporate gaseous surface contaminations such as carbon monoxide, oxygen or water. As described in Section 3.2 .3 later experiments with heating and sputtering showed that this approach reduces the MIT contrast of $\mathrm{VO}_{2}$ films significantly. Which means that if these samples ever had a MIT it was probably destroyed during the annealing.

After the scattering experiments AES spectra were measured of CVD2 to analyze the elemental composition of the surface. This is shown in Figure 4.2. The size of the carbon signal visible in the spectrum is comparable to the amount of vanadium and oxygen. This means that a significant portion of the surface is probably covered with carbon during the scattering experiments.

CVD1 and CVD2 were used for scattering experiments with $\mathrm{NO} \mathrm{X}^{2} \Pi(v=11, J=0.5)$ to verify if direct scattering can occur from surfaces with a rough $\mathrm{VO}_{2}$ film. While neither surface showed the desired MIT they can still give insights about scattering from rough surfaces. 


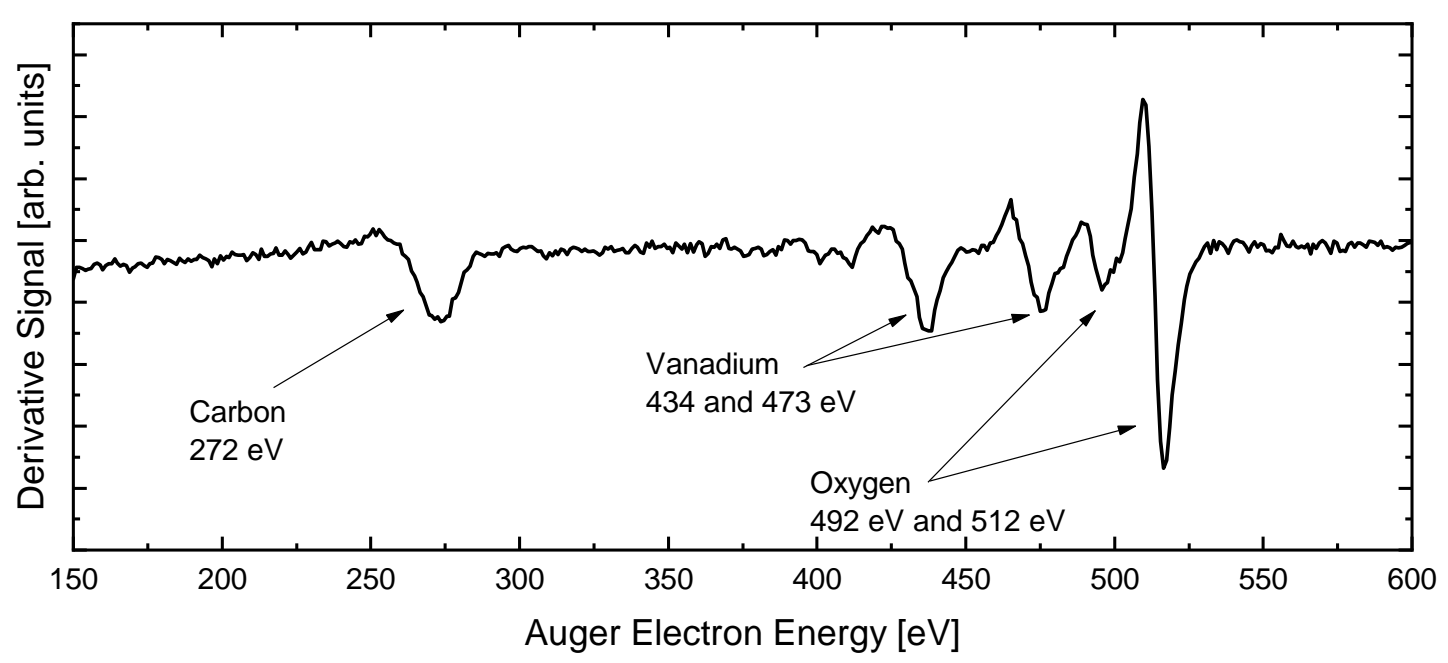

Figure 4.2: Auger Electron Spectrum of CVD2 taken after scattering experiments. Carbon contamination of the surface is shown by the signal at $272 \mathrm{eV}$.

\subsubsection{Angular Distribution of Scattered NO}

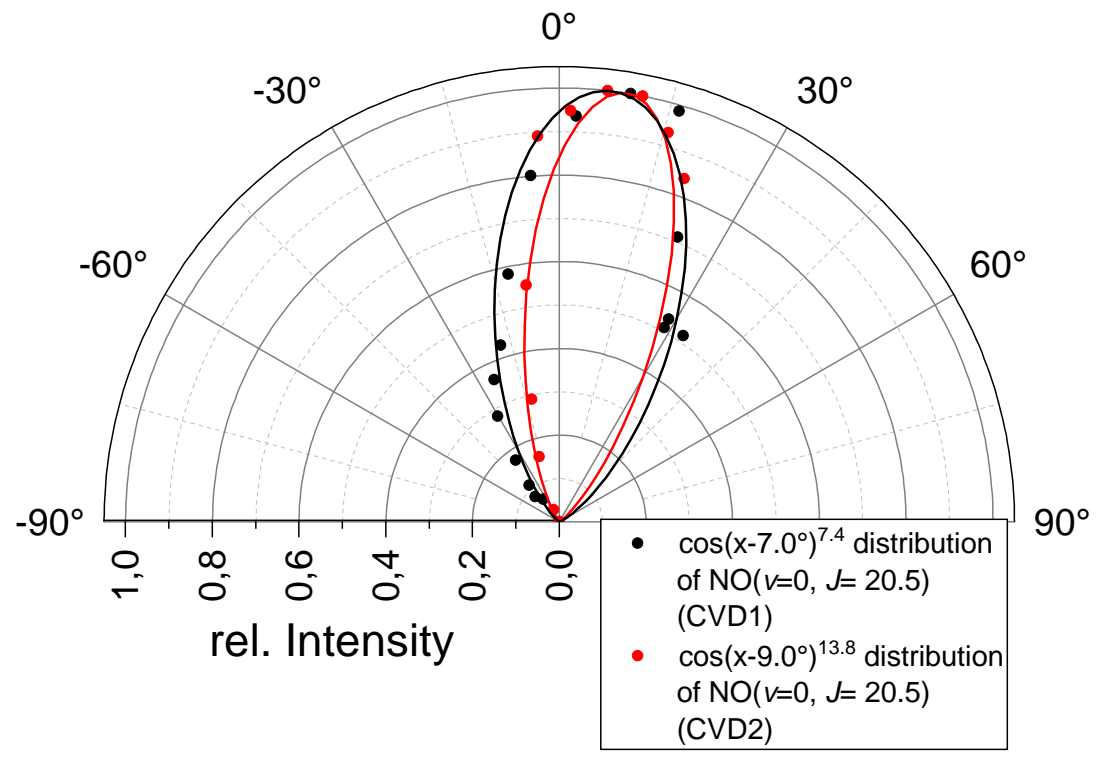

Figure 4.3: Angular distribution of the $\mathrm{Q}_{11}(J=20.5)$ line of the $\gamma(0,0)$ band after scattering from CVD1 (black) and CVD2 (red).

Angular distributions $\mathrm{NO}(v=11)$ the wavelength of the $\mathrm{Q}_{11}(J=20.5)$ line of the $\gamma(0,0)$ transition band were recorded for both surfaces. The results are shown in Figure 4.3 One can see two narrow angular distributions. For CVD1 the distribution is fitted with a $\cos ^{7.4}$ function and for CVD2 it is fitted with a $\cos ^{13.8}$ function. The 
narrow angular distributions are an indicator for direct scattering. CVD1 appears to have a wider distribution than CVD2. This is caused by the fact that CVD2 has a flatter surface while CVD1 consists of several visible flakes with slightly different angles in relation to the molecular beam. This widens the distribution because its composed of several overlaid components with different angular offsets. The samples were placed perpendicular to the molecular beam. Due to deformation during heating and inaccuracy during the mounting the samples are slightly shifted resulting in an angular distribution that is not centered at $0^{\circ}$.

\subsubsection{Time-of-Flight Distributions of Scattered NO}

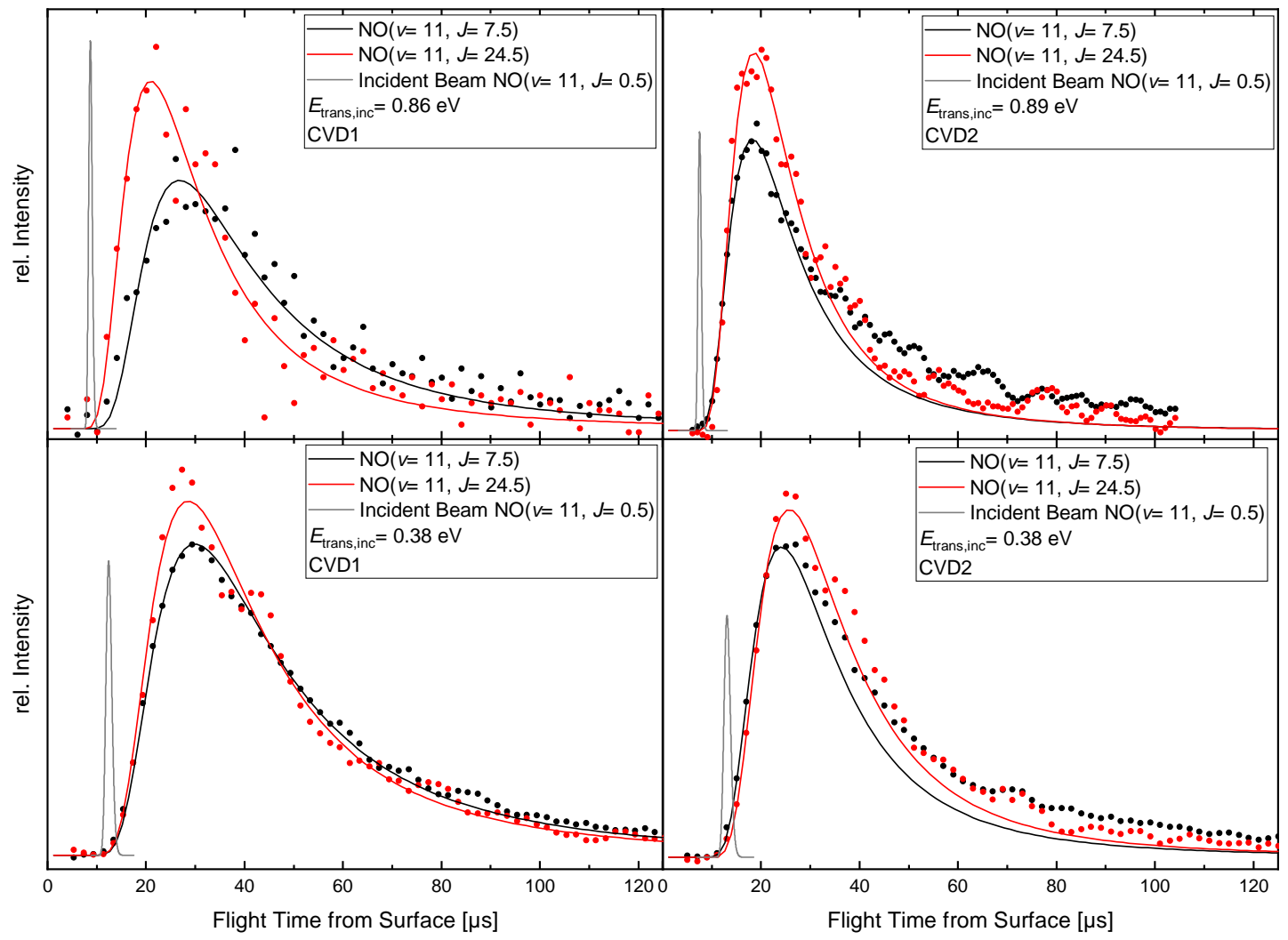

Figure 4.4: Time-of-Flight distributions (points) for high and low translational energy of the incident molecular beam $E_{\text {inc }}$ of the $\mathrm{Q}_{11}(7.5)$ and $\mathrm{Q}_{11}(24.5)$ line of the $\gamma(3,11)$ transition band scattered from CVD1 and CVD2 with direct scattering TOF distribution functions fitted to them (line plot). The TOF distribution of $\mathrm{NO}(v=11, J=0.5)$ in the incident molecular beam is shown as a grey line.

TOF distributions at the wavelengths of the $\mathrm{Q}_{11}(7.5), \mathrm{Q}_{11}(10.5), \mathrm{Q}_{11}(16.5), \mathrm{Q}_{11}(19.5)$, $\mathrm{R}_{11}(24.5), \mathrm{Q}_{11}(31.5)$ transitions of the $\gamma(3,11)$ band were measured. They are analyzed 


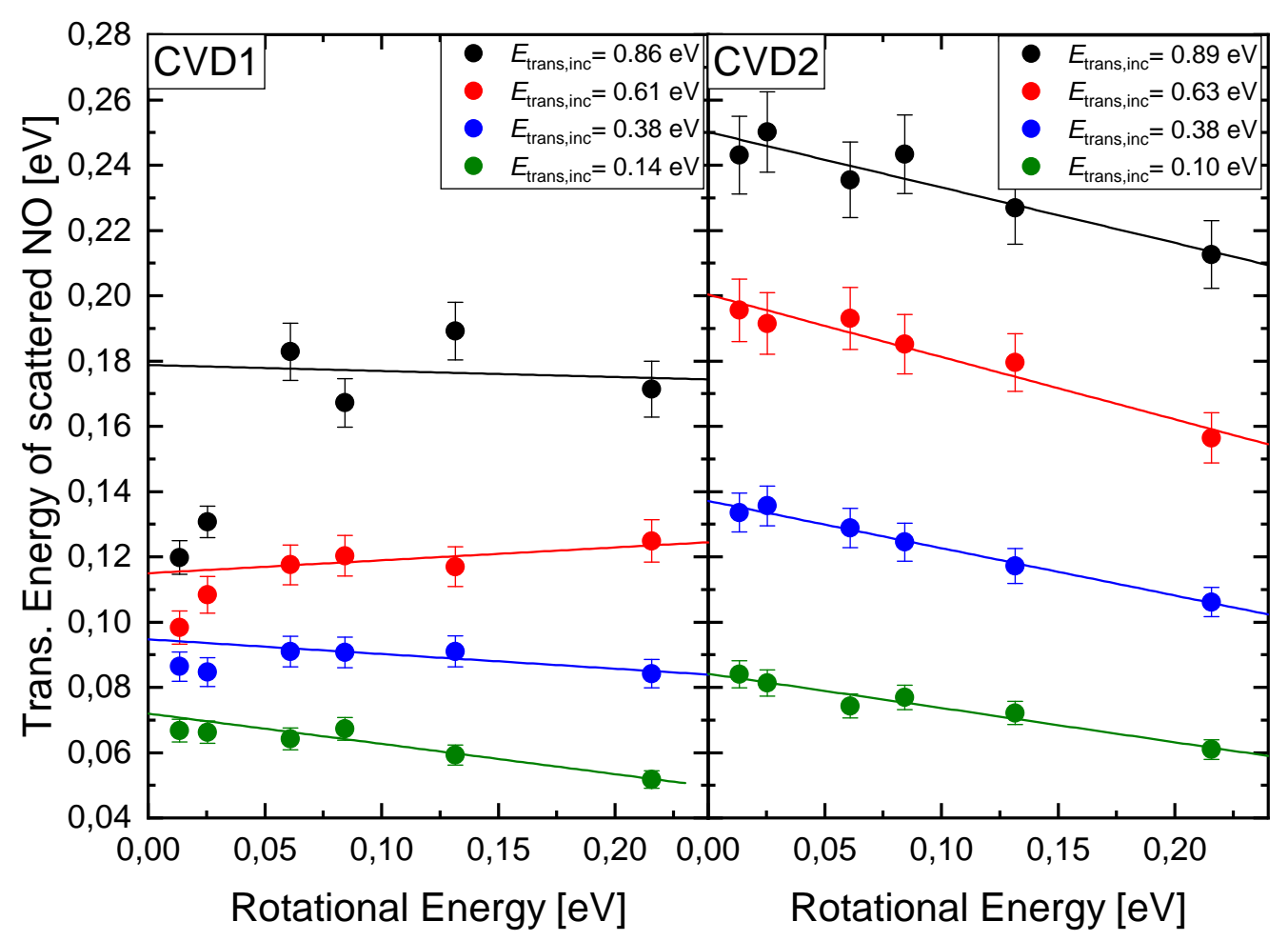

Figure 4.5: Translational energies of the direct scattering TOF distributions for all rotational transition lines measured from CVD1 (left) and CVD2 (right) plotted against the rotational energy of the rotational levels $J=7.5, J=10.5, J=16.5, J=19.5, J=24.5$ and $J=31.5$. Each set of TOFs is fitted with a linear function to extrapolate it to $J=0$.

for the translational energy of the NO molecules scattered from both CVD1 and CVD2. The results for CVD1 are listed in Table 4.1 and the results for CVD2 are listed in Table 4.2. Exemplary TOF distributions for CVD1 and CVD2 are shown in Figure 4.4. The shape, width and flight time of the TOF distributions indicate on first sight that the molecules are scattered directly. The shape of the TOF distribution is almost perfectly matched by the fitted direct scattering TOF function shown in Equation 3.5. Only for CVD2 a tail component arises at arrival times $>50 \mu \mathrm{s}$. The TOF distributions of CVD2 can be fitted by adding a trapping desorption term (Equation 3.6) to the fitting function. The tail component becomes more pronounced for NO molecules in lower $J$ states such as $\mathrm{NO}(J=7.5)$ shown on the right side of Figure 4.4 .

TOF distributions of NO scattered from CVD1 appear to consist only of a direct scattering component. The TOF distributions of NO scattered from CVD1 have a significantly larger beam spread than the corresponding TOF distributions of NO scattered from CVD2. Especially for the low rotational state $J=7.5$ the distributions of NO 
Table 4.1: Mean translational energy of TOF distributions of $\mathrm{NO}(v=11)$ scattered from CVD1.

\begin{tabular}{ccccc}
\hline Rovibrational State & $\begin{array}{c}\langle E\rangle_{\text {trans }} \\
\text { in meV }\end{array}$ & $\begin{array}{c}\langle E\rangle_{\text {trans }} \\
\text { in meV }\end{array}$ & $\begin{array}{c}\langle E\rangle_{\text {trans }} \\
\text { in meV }\end{array}$ & $\begin{array}{c}\langle E\rangle_{\text {trans }} \\
\text { in meV }\end{array}$ \\
\hline Incident $\mathrm{NO}(v=11, J=0.5)$ & $860 \pm 50$ & $610 \pm 40$ & $380 \pm 30$ & $140 \pm 10$ \\
$(v=11, J=7.5)$ & $120 \pm 6$ & $98 \pm 6$ & $86 \pm 5$ & $67 \pm 4$ \\
$(v=11, J=10.5)$ & $131 \pm 5$ & $108 \pm 6$ & $85 \pm 5$ & $66 \pm 4$ \\
$(v=11, J=16.5)$ & $182 \pm 9$ & $118 \pm 7$ & $91 \pm 5$ & $64 \pm 4$ \\
$(v=11, J=19.5)$ & $167 \pm 8$ & $120 \pm 7$ & $91 \pm 5$ & $67 \pm 4$ \\
$(v=11, J=24.5)$ & $189 \pm 9$ & $117 \pm 7$ & $91 \pm 5$ & $59 \pm 4$ \\
$(v=11, J=31.5)$ & $171 \pm 9$ & $125 \pm 7$ & $84 \pm 5$ & $52 \pm 3$ \\
\hline
\end{tabular}

scattered from CVD1 are about twice as wide than those observed for CVD2. Since both measurements were done on the same apparatus with identical parameters this difference in width must be caused by the surface. Furthermore, TOF distributions of NO scattered from CVD1 show some differences regarding their relative position along flight time axis compared to NO scattered from CVD2. In both plots shown on the left side of Figure 4.4 the peak of the TOF distributions of $\mathrm{NO}(v=11, J=7.5)$ arrives later than the peak of the distributions of $\mathrm{NO}(v=11, J=24.5)$. This behavior can be seen especially pronounced for the faster incident molecular beam with $0.86 \mathrm{eV}$ translational energy. For directly scattered NO molecules one would expect an anti-proportional dependence, because during the scattering event translational energy is converted to rotational excitation ${ }^{42}$. So the more rotationally excited a molecule is after scattering, the less translational energy it should have. This behavior reminiscent of direct scattering can be seen in NO scattered from CVD2.

The difference in behavior between CVD1 and CVD2 can be seen more clearly in Figure 4.5. On the right side of the figure the translational energies of scattered NO are plotted against their rotational energy. It can be seen that for all probed translational energies of the incident molecular beam the translational energy of scattered NO follows a linear progression with negative slope along the rotational energy axis.

Similar plots of translational energy against rotational energy are shown for NO scattered from CVD1 on the left side of the same figure. It can be seen that only for low translational energies of incident NO and for high $J$ values the points follow linear progressions with a negative slope. For all translational energies but $0.14 \mathrm{eV}$ the two 
Table 4.2: Mean translational energy of TOF distributions of $\mathrm{NO}(v=11)$ scattered from CVD2.

\begin{tabular}{ccccc}
\hline Rovibrational State & $\begin{array}{c}\langle E\rangle_{\text {trans }} \\
\text { in meV }\end{array}$ & $\begin{array}{c}\langle E\rangle_{\text {trans }} \\
\text { in meV }\end{array}$ & $\begin{array}{c}\langle E\rangle_{\text {trans }} \\
\text { in meV }\end{array}$ & $\begin{array}{c}\langle E\rangle_{\text {trans }} \\
\text { in meV }\end{array}$ \\
\hline Incident $\mathrm{NO}(v=11, J=0.5)$ & $890 \pm 50$ & $640 \pm 40$ & $380 \pm 30$ & $100 \pm 10$ \\
$(v=11, J=7.5)$ & $243 \pm 12$ & $196 \pm 10$ & $134 \pm 6$ & $84 \pm 5$ \\
$(v=11, J=10.5)$ & $250 \pm 13$ & $192 \pm 10$ & $136 \pm 7$ & $81 \pm 4$ \\
$(v=11, J=16.5)$ & $236 \pm 12$ & $193 \pm 10$ & $129 \pm 6$ & $74 \pm 4$ \\
$(v=11, J=19.5)$ & $243 \pm 13$ & $185 \pm 10$ & $125 \pm 6$ & $77 \pm 4$ \\
$(v=11, J=24.5)$ & $227 \pm 12$ & $179 \pm 9$ & $117 \pm 6$ & $72 \pm 4$ \\
$(v=11, J=31.5)$ & $212 \pm 11$ & $156 \pm 8$ & $106 \pm 5$ & $61 \pm 3$ \\
\hline
\end{tabular}

lowest probed $J$ states drop out of the linear progression. Furthermore, the slopes of the linear progressions for CVD1 are smaller compared to CVD2. The translational energy of $0.61 \mathrm{eV}$ in red shows even a positive slope. In general, the loss of translational energy during the scattering is about $30 \%$ higher when scattered from CVD1 than for scattering of CVD2.

This is a strong indicator that the TOF distributions of NO scattered from CVD1 consist of another non-direct scattering component which overlays the direct scattering component. Especially in low $J$ states this non-direct scattering component is of comparable intensity to the direct scattering component. This results in an uncharacteristically broad TOF distribution where the direct scattering component can not be resolved.

Figure 4.6 shows the Baule plot drawn with the for rotational excitation adjusted translational energies from the TOF analysis. With the mass of NO $m_{\mathrm{NO}}=30 \mathrm{amu}$ and the slope from the linear progression shown in Figure 4.6 the effective surfaces masses of $m_{\mathrm{CVD} 1}=48 \mathrm{amu}$ and $m_{\mathrm{CVD} 2}=81 \mathrm{amu}$ are gained. The mass of CVD2 is very close to the mass of $\mathrm{VO}_{2}$ with $2 \cdot m(\mathrm{O})+m(\mathrm{~V})=(2 \cdot 16+51) \mathrm{amu}=83 \mathrm{amu}$. In the case of CVD1 it is significantly off. This is an indication that the surface composition of CVD1 is different than the surface composition of CVD2. 


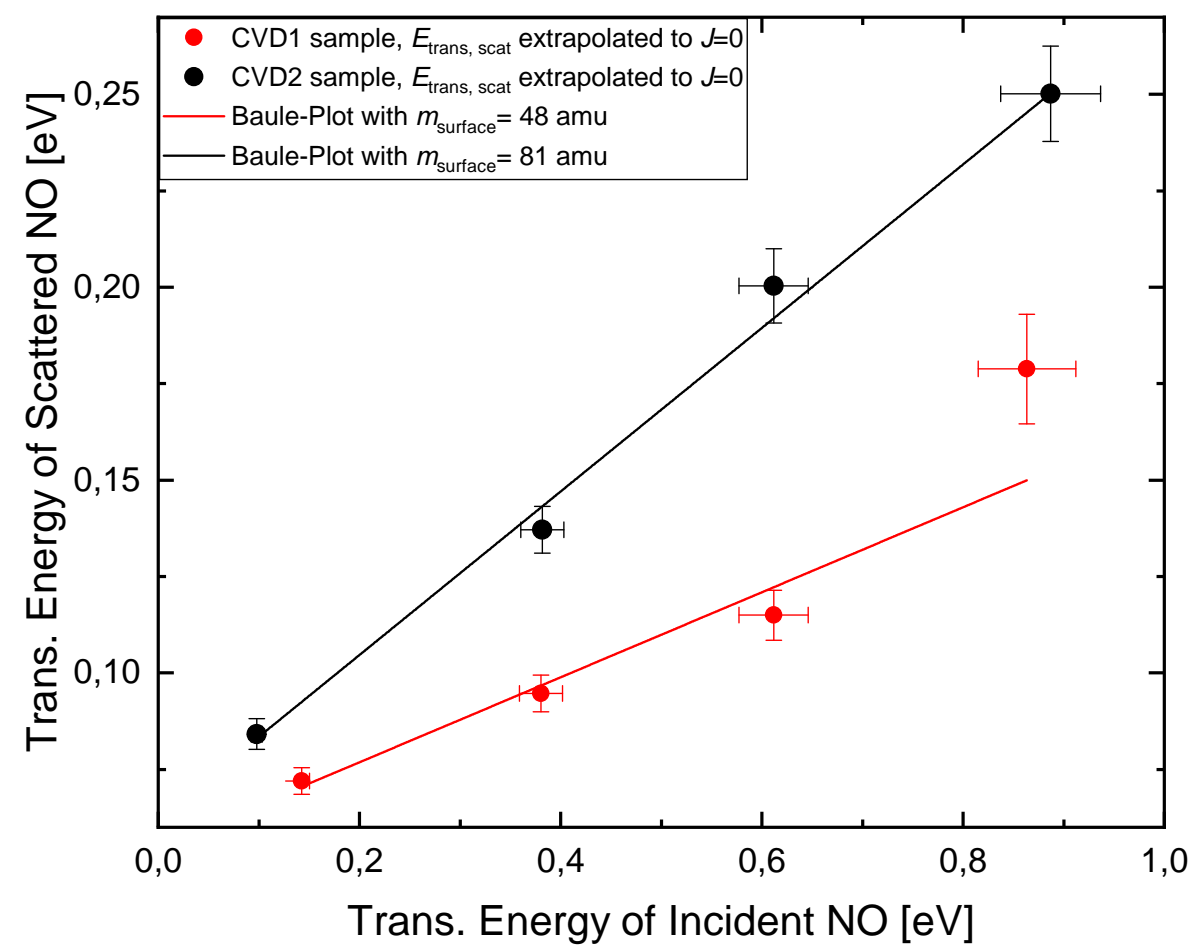

Figure 4.6: Baule plot of the dependence between the translational energy of the incident NO and the scattered NO. The points correspond to $J=0$ extrapolation of the data shown in Figure 4.5

\section{Conclusions}

The TOF experiments show that for NO scattering from CVD1 and CVD2 the direct channel is the dominant channel. Yet, for both CVD1 and CVD2 non-direct components can be observed in the TOF distributions.

For NO scattered from CVD2 the non-direct scattering component emerges as a tail next to the direct scattering component at later flight times. Its intensity is, compared to the direct scattering component, small so that it does not overshadow the direct scattering component. This makes it possible to analyze the directly scattered NO molecules for their translation energy.

For NO scattered from CVD1 the non-direct scattering component's intensity is comparable to the direct scattering component. In consequence, the direct scattering component is not resolvable for some $J$ states and translational energies can not be determined for these NO molecules.

In both cases the intensity of the non-direct scattering component is higher for lower $J$ states. This can be explained by the fact that the NO molecules of the non-direct 


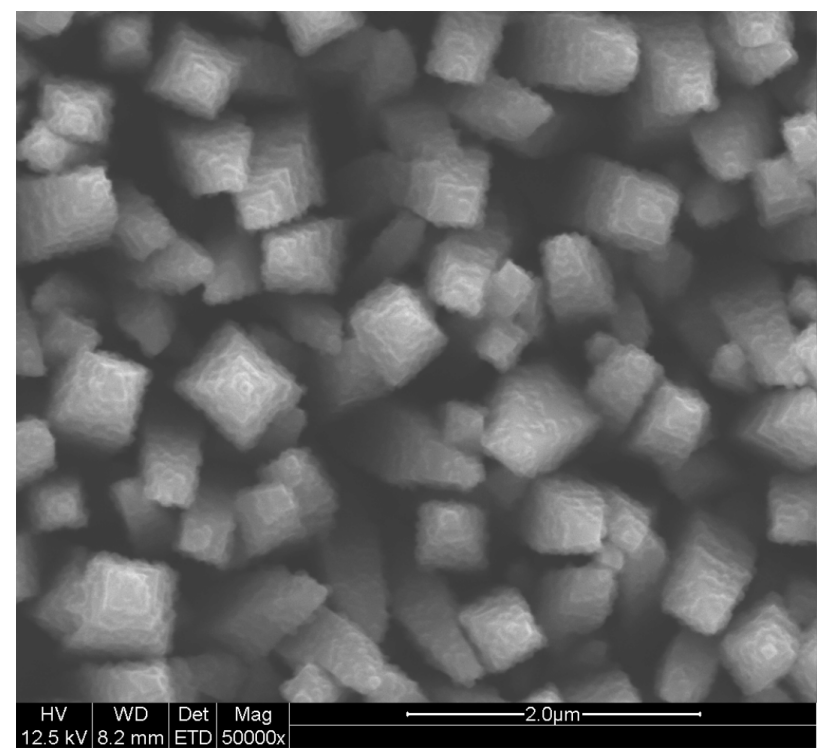

Figure 4.7: Environmental scanning electron microscope (ESEM) image of a CVD prepared $\mathrm{VO}_{2}$ film. It shows that the surface consists of various grains with less than $1 \mu \mathrm{m}$ diameter. It is assumed that NO molecules are trapped between the grains. This results in multiple surface collision and partial equilibration with the surface degrees of freedom. The brightness indicates the height of the surface features. Brighter pixels are closer to the observer than the dark pixels.

scattering component equlibrate more with the surface. The surface temperature of $\approx 300 \mathrm{~K}$ then results in increased populations of lower $J$ states in the non-directly scattered molecules.

The mechanism of this non-direct scattering component can be either trapping desorption, a multi-bounce scattering event or a combination of both. From the environmental scanning electron microscope (ESEM) image of a CVD prepared $\mathrm{VO}_{2}$ film shown in Figure 4.7 it can be speculated that NO molecules might penetrate into the gaps between the $\mathrm{VO}_{2}$ grains of the surface. It can be assumed that faster molecules penetrate with a higher probability than slower molecules. There they bounce multiple times before leaving. During this they equilibrate to the surface degrees of freedom. CVD2 consists of condensed individual grains of $\mathrm{VO}_{2}$ that are homogeneously spread over the substrate. It is thinkable that NO might also get trapped in the gaps between the grains and get equilibrated through multiple collisions before it leaves. This has a lower probability on CVD2 than on CVD1 so the TOF distributions of NO scattered from CVD2 only shows a small tail.

The results from the Baule plot for the effective mass of CVD2 $(m($ CVD2 $)=81 \mathrm{amu})$ are close to the mass of the $\mathrm{VO}_{2}$ unit $\left(m\left(\mathrm{VO}_{2}\right)=83 \mathrm{amu}\right)$. This is probably coincidental 
because the $\mathrm{VO}_{2}$ lattice does not consist of isolated $\mathrm{VO}_{2}$ units but all particles in the lattice are interconnected through chemical bonds. Therefore, NO is not expected to scatter from an isolated $\mathrm{VO}_{2}$ unit. The fact that the effective mass of CVD1 is different from the effective mass of CVD1, exemplifies how CVD preparation can result in very different surfaces and that films prepared this way are not necessarily comparable.

In summary it can be said that direct scattering with CVD prepared samples can be observed under certain circumstances. TOF distribution analysis of NO scattered from CVD1 showed some issues with higher translational energies of the incident molecular beam pulse and for lower $J$ states of scattered NO. In these cases a single direct scattering component in the TOF distributions could not be resolved. It is reasonable to assume that this is caused by the rough surface structure.

\subsubsection{REMPI Spectra of Scattered NO}

REMPI spectra of NO scattered in the vibrational and ground electronic state and in the highly vibrationally excited ground electronic state $\mathrm{NO}(v=11)$ were measured at different translational energies of the the incident molecular beam. Exemplary spectra of both initial vibrational states of NO in its raw and background corrected form and with corresponding fitted simulation spectra are shown in Figure 4.8. It can be seen that the experimental data is well reproduced by the fitted simulation spectrum. It also shows that, while a wide rotational distribution up to $J=40.5$ is generated during the collision, NO remains almost exclusively in its initial vibrational state when scattered from a $\mathrm{VO}_{2}$ surface at room temperature. In the REMPI spectra of scattered $\mathrm{NO}(v=11)$ next to the $\gamma(3,11)$ band the $\gamma(1,8)$ band can be detected with lower intensity. It is overshadowed by the high $J$ lines of the $\gamma(3,11)$ band. In Figure 4.8 (c) one can see that most of the $\gamma(3,11)$ transition band is generated from spontaneous emission from the electronically excited $\mathrm{A}^{2} \Sigma^{+}(v=2, J=0.5)$ state. Therefore, if any $\mathrm{NO}(v=11)$ relaxes down to the $(v=8)$ state it is reasonable to assume that the amount is very small compared to the relaxation one can observe from scattering $\operatorname{NO}(v=11)$ from $\mathrm{Au}(111)$ 41].

The rotational temperatures of the various REMPI spectra taken from NO scattered from CVD1 and CVD2 are plotted against the translational energy of the incident molecular beam in Figure 4.9. The corresponding numbers are listed in Table 4.3. Rotational temperatures that belong to the same translational energy are averaged for this plot. All sets of rotational temperatures of $\mathrm{NO}(v=0)$ and $\mathrm{NO}(v=11)$ scattered from CVD1 and CVD2 show a linear progression with positive slope along the translational energy of the incident molecular beam pulse. 


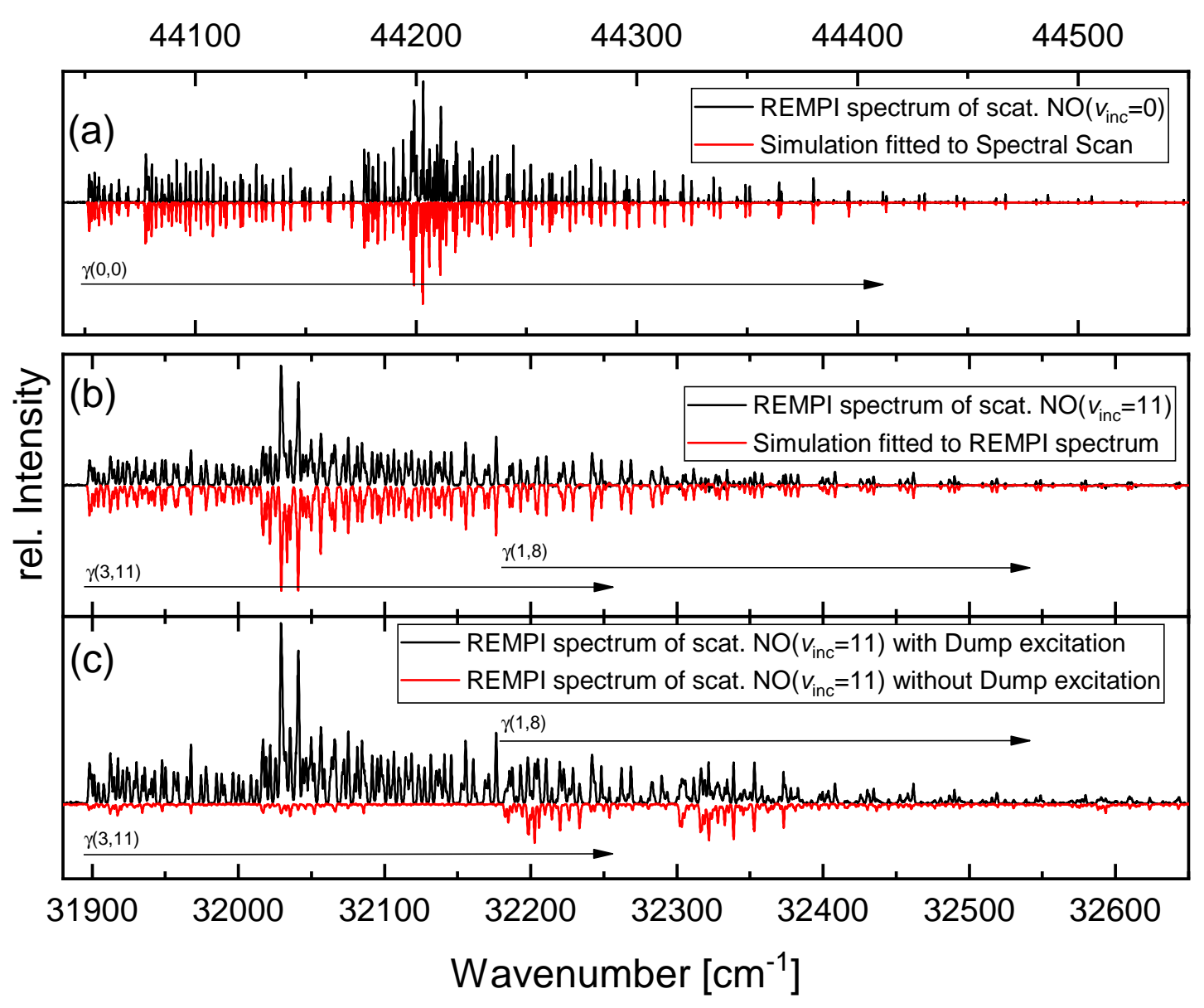

Figure 4.8: Different exemplary REMPI spectra of NO scattered with $E_{\text {trans,inc }}=0.6 \mathrm{eV}$ from CVD2. Panel (a) shows the experimental REMPI spectrum of $\mathrm{NO}\left(v_{\text {inc }}=0\right.$ ) (black line) and a simulated spectrum fitted to the experimental data (red line). Panel (b) shows the experimental background corrected REMPI spectrum of $\mathrm{NO}\left(v_{\text {inc }}=11\right)$ (black line) and a simulated spectrum fitted to the experimental data (red line). Panel (c) shows the REMPI spectrum of the $\mathrm{NO}\left(v_{\text {inc }}=11\right)$ molecular beam prepared in a Pump-Dump scheme (black line) and the corresponding background of the same molecular beam without the Dump deexcitation. 


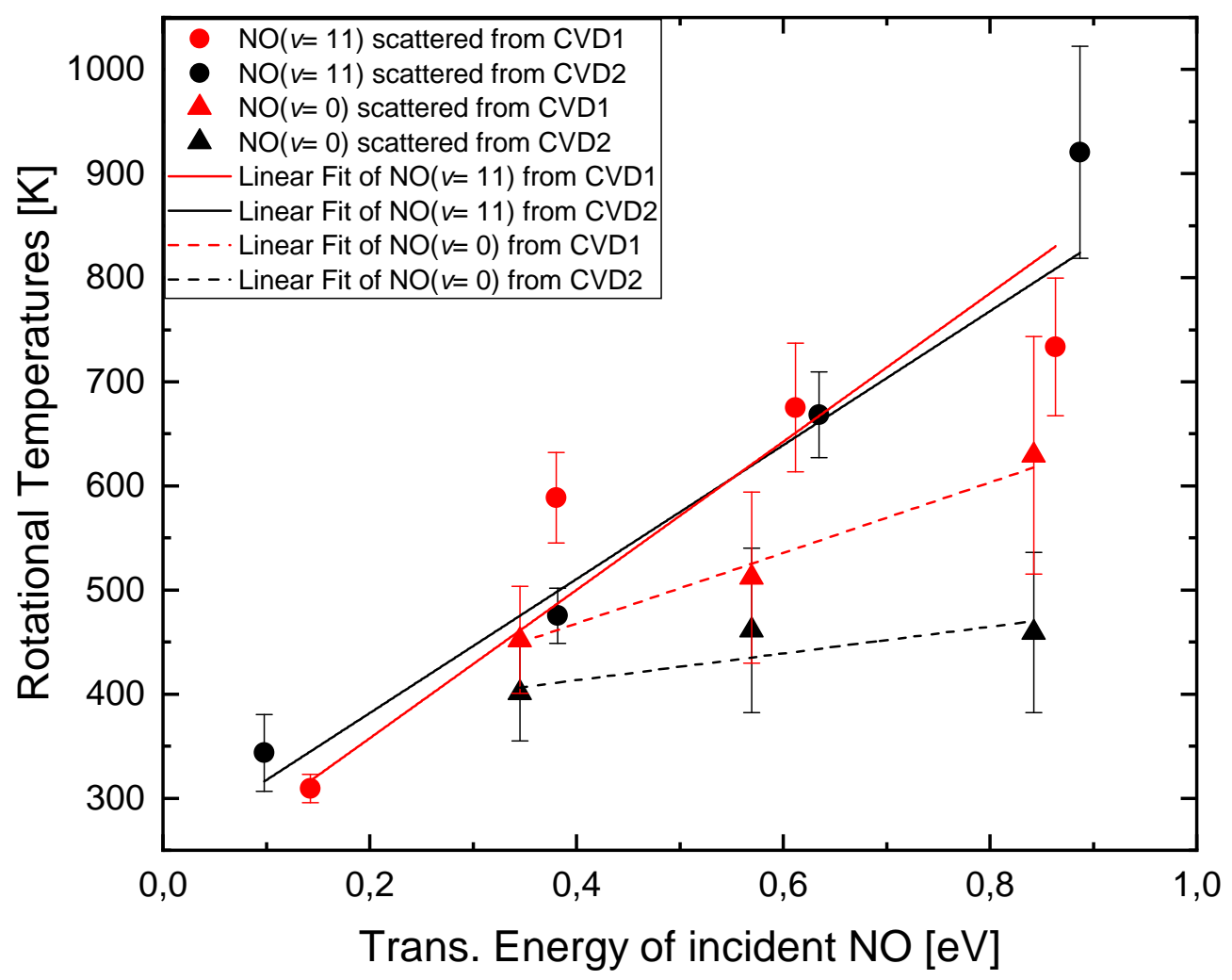

Figure 4.9: Rotational temperatures of scattered NO. The initial vibrational states $v=0$ and $v=11$ are probed and scattered from CVD1 and CVD2. One has to note that the rotational temperatures of $v=0$ and $v=11$ are not comparable. For $v=11$ only a small cut from the molecular beam pulse with a relatively narrow velocity spread is observed. In the case of $v=0$ no excitation occurs so the whole molecular beam is observed. The molecular beam consists of $\mathrm{NO}(v=0)$ molecules with a large range of different velocities that generate different rotational excitation when scattered.

Furthermore, all measured rotational temperatures of NO molecules scattered with translational energies larger than $0.3 \mathrm{eV}$ are significantly higher than the surface temperature of $T_{\text {Surface }} \approx 300 \mathrm{~K}$. The linear progressions of rotational temperature of $\mathrm{NO}(v=11)$ scattered from CVD1 and CVD2 are with regard to the error bars and the deviation of the single points almost identical. The rotational temperatures corresponding to $\mathrm{NO}(v=0)$ scattered from CVD1 are smaller than those of $\mathrm{NO}(v=11)$. Those corresponding to scattering from CVD2 are even lower. Again, with regard of the error bars the difference between those two surfaces can be considered negligible. Also the slope of the linear progression of the rotational temperatures of $\mathrm{NO}(v=0)$ is smaller than for $\mathrm{NO}(v=11)$. 
Table 4.3: Rotational temperatures $T_{\text {rot }}$ of the $\gamma(3,11)$ and $\gamma(0,0)$ transition band of NO scattered from CVD1 and CVD2.

\begin{tabular}{cccc} 
CVD1 & \multicolumn{3}{c}{ CVD2 } \\
\hline$\langle E\rangle_{\text {trans,inc }}$ & $T_{\text {rot }}(v=11)$ & $\langle E\rangle_{\text {trans,inc }}$ & $T_{\text {rot }}(v=11)$ \\
in meV & in K & in meV & in K \\
\hline 860 & $730 \pm 70$ & 890 & $920 \pm 100$ \\
610 & $680 \pm 70$ & 640 & $670 \pm 50$ \\
380 & $590 \pm 50$ & 380 & $480 \pm 30$ \\
140 & $310 \pm 20$ & 100 & $340 \pm 40$ \\
\hline$\langle E\rangle_{\text {trans,inc }}$ & $\langle E\rangle_{\text {rot }}(v=0)[\mathrm{meV}]$ & $\langle E\rangle_{\text {trans,inc }}$ & $T_{\text {rot }}(v=0)$ \\
in meV & in K & in meV & in K \\
\hline 840 & $630 \pm 120$ & 840 & $460 \pm 80$ \\
570 & $510 \pm 90$ & 570 & $460 \pm 80$ \\
350 & $450 \pm 60$ & 350 & $400 \pm 50$ \\
\hline
\end{tabular}

\section{Conclusions}

Similar to the TOF distributions the results from the REMPI spectrum analysis also indicate direct scattering from CVD1 and CVD2.

The low relaxation probability of $\mathrm{NO}\left(v_{\text {inc }}=11\right)$ during the collision with both surfaces indicates that the interaction time between molecule and surface is too small for vibrational equilibration. Furthermore, it shows that the coupling between the molecular vibrational quanta and electron-hole pairs of the surface, that is essential for the occurrence of vibrational relaxation of NO on metal surfaces, is weak. This suggests that both CVD1 and CVD2 do not behave like metal surfaces in this regard. This is conceivable since the surface are held at room temperature where $\mathrm{VO}_{2}$ is in its monoclinic phase below the MIT with high resistance. Also, it is likely that it is at least partially covered with carbon as shown in Figure 4.2 .

The high rotational temperatures draw the same picture. The NO molecules show very low equilibration with the surface, which indicates direct scattering from CVD1 and CVD2. The incidence translational energy of the NO molecules is transferred to rotational excitation during the collision. Only in the case of translational energies close to the rotational energy the rotational temperature is found close to room temperature. For all translational energies the rotational temperature is significantly higher 
than room temperature. Furthermore, it scales linearly with the translational energy. While not mentioned in the paragraphs above the surface was heated several times to $100{ }^{\circ} \mathrm{C}$ and back to room temperature. Along this temperature ramp the intensity of different transition lines of the $\gamma(3,11)$ band was measured. In no case it was possible to observe a sudden change in intensity over the temperature neither at the MIT temperature nor at any other temperature. This absence of dependence between relaxation probability of $\mathrm{NO}(v=11)$ and MIT temperature is attributed to the broken state of the surface that have been heated to $400{ }^{\circ} \mathrm{C}$ for extended periods of time and therefore showed no MIT in the resistance measurement against temperature. Also significant carbon contaminations covered the surface so it can not be excluded that NO was at least partially scattered from carbon instead of $\mathrm{VO}_{2}$.

\subsection{Scattering of $\mathrm{NO}(v=2)$ from CVD3}

\subsubsection{Sample Characteristics of CVD3}

With the insights from NO scattering from CVD1 and CVD2 another CVD prepared $\mathrm{VO}_{2}$ film was chosen for follow up $\mathrm{NO}$ scattering experiments. In the following it will be labeled as CVD3. From all the, at that time, available CVD prepared $\mathrm{VO}_{2}$ it showed the most pronounced MIT. An image of the sample is shown in Figure 4.10 (b). It shows the surface before it was placed into the sample mount. In contrast to CVD1 and CVD2, CVD3 was prepared on a $\mathrm{Si}$ substrate instead of $\mathrm{SiO}_{2}$. The substrate material can be seen as the upper rectangular part of the sample with a darker shade. The lower grey part is the $\mathrm{VO}_{2}$ film which shows the same rough grained structure as CVD2. The surface bears minor scratches at the edges and four point marks from the 4-Probe device contacts at its center.

For the experiments with CVD3 the UHV chamber was modified and a UHV compatible 4-Probe was added. Figure 4.10 (a) shows the results from the 4-Probe measurement right after the sample was placed in the UHV as a dashed line. The solid line in the same figure shows a 4-Probe measurement that was taken directly before the scattering experiments. An initial MIT contrast of about 820 was measured at a transition temperature of $64^{\circ} \mathrm{C}$. It was reduced to 220 after the $\mathrm{Ar}^{+}$-ion sputtering procedure.

For surface preparation the surface was sputtered two times for 10 min with $\mathrm{Ar}^{+}$-ions accelerated to $1 \mathrm{kV}$ before the day of the scattering experiments. On the experiments day it was sputtered for an additional $10 \mathrm{~min}$ with $\mathrm{Ar}^{+}$-ions accelerated to $1 \mathrm{kV}$. The AES before and after surface preparation are shown in Figure 4.10 (c). It is apparent that while the carbon signal intensity is significantly reduced compared to the initial 

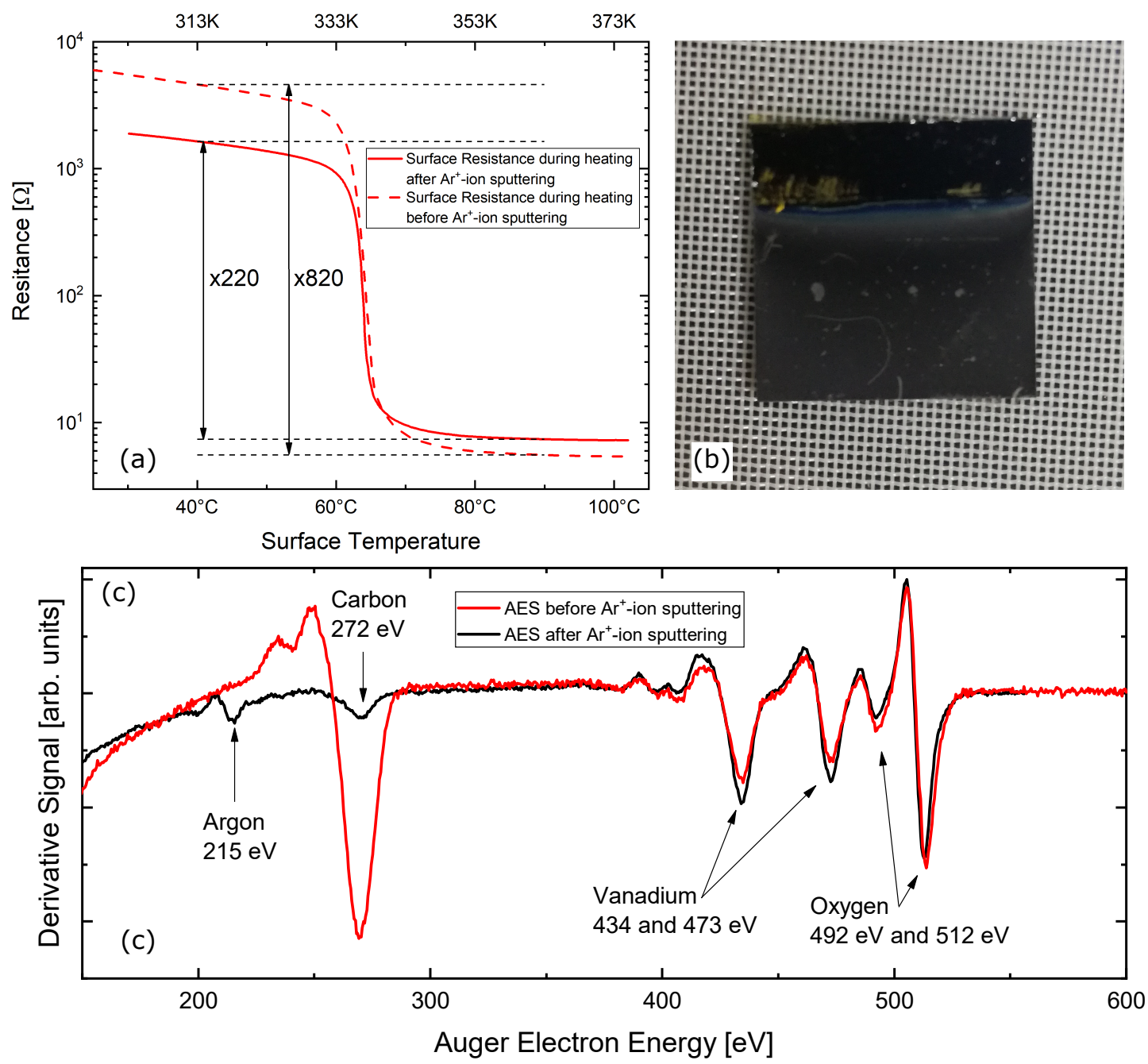

Figure 4.10: Sample characteristics of CVD3. (a) shows the surface resistance against temperature plot of CVD3 before and after sputtering with $1 \mathrm{kV} \mathrm{Ar}{ }^{+}$-ions. (b) shows an image of the surface with the dark upper part being uncovered substrate and the lower grey part being the $\mathrm{VO}_{2}$ film. (c) shows the AES of CVD3 before and after sputtering.

carbon signal it is still clearly visible after sputtering. Furthermore, next to it is another signal that only arises after sputtering and can be attributed to argon. It is assumed that the signal is generated by argon from the sputtering procedure which has penetrated the surface and is now trapped in the crystal lattice. Usually this cannot be seen in sputtered metal single crystal because it evaporates during annealing. In the case of $\mathrm{VO}_{2}$ the trapped argon can not be eliminated since the surface can not be heated above $100{ }^{\circ} \mathrm{C}$ without damaging the surface composition. 
Table 4.4: Mean translational energy of $\operatorname{NO}(v=11)$ scattered from CVD3..

\begin{tabular}{ccc}
\hline & $\langle E\rangle_{\text {trans }}$ & $\langle E\rangle_{\text {trans }}$ \\
Rovibrational State & $\begin{array}{c}T_{\text {surf }}=25^{\circ} \mathrm{C} \\
\text { in meV }\end{array}$ & $\begin{array}{c}T_{\text {surf }}=80^{\circ} \mathrm{C} \\
\text { in meV }\end{array}$ \\
\hline Incident $\mathrm{NO}(v=2, J=0.5)$ & $980 \pm 10$ & $980 \pm 10$ \\
$(v=2, J=6.5)$ & $190 \pm 17$ & $195 \pm 18$ \\
$(v=2, J=8.5)$ & $190 \pm 17$ & $199 \pm 18$ \\
$(v=2, J=16.5)$ & $186 \pm 17$ & $189 \pm 18$ \\
$(v=2, J=31.5)$ & $168 \pm 15$ & $175 \pm 16$ \\
\hline
\end{tabular}

To increase the chance to see MIT related effects on the scattered NO a different, less complex laser excitation scheme was used with CVD3. This was done by switching from SEP preparation of $\mathrm{NO}(v=11)$ to overtone pumping preparation of $\mathrm{NO}(v=2)$. This technique allows the preparation of high intensity molecular beam pulses of $\mathrm{NO}(v=2)$ with low background signal and overall reduced power fluctuations due to the fact that this molecular beam preparation only requires one laser beam instead of two.

With CVD3 only one gas mixture of $1 \% \mathrm{NO}$ in $\mathrm{H}_{2}$ with a translational energy of about $0.98 \mathrm{eV}$ was used. From earlier experiments 41 it is known that vibrational relaxation in NO increases with higher translational energy of incident NO molecules. For this reason and since direct scattering with fast gas mixture was observed by scattering NO from CVD2 the fastest available gas mixture was used.

\subsubsection{Time-of-Flight Spectroscopy of Scattered NO}

The TOF distributions of $\mathrm{NO}(v=2)$ scattered from CVD3 consist in all cases of a fast and a slow component. The fast component has a distinct narrow distribution and is visible for all measured rotational lines as shown in Figure 4.11. This component is fitted perfectly by the function shown in Equation 3.5 and has a width of about $10 \mu \mathrm{s}$ for all probed transition lines. Due to its position and shape in the TOF measurement it is attributed to the direct scattering channel. At later flight times another component arises that is fitted by the function shown in Equation 3.6. The results from the analysis of the TOF distributions are listed in Table 4.4.

The slow component is more intense for lower $J$ states than for higher $J$ states. For $J=31.5$ the intensity of the slow component is only seen as a tail component of the TOF 

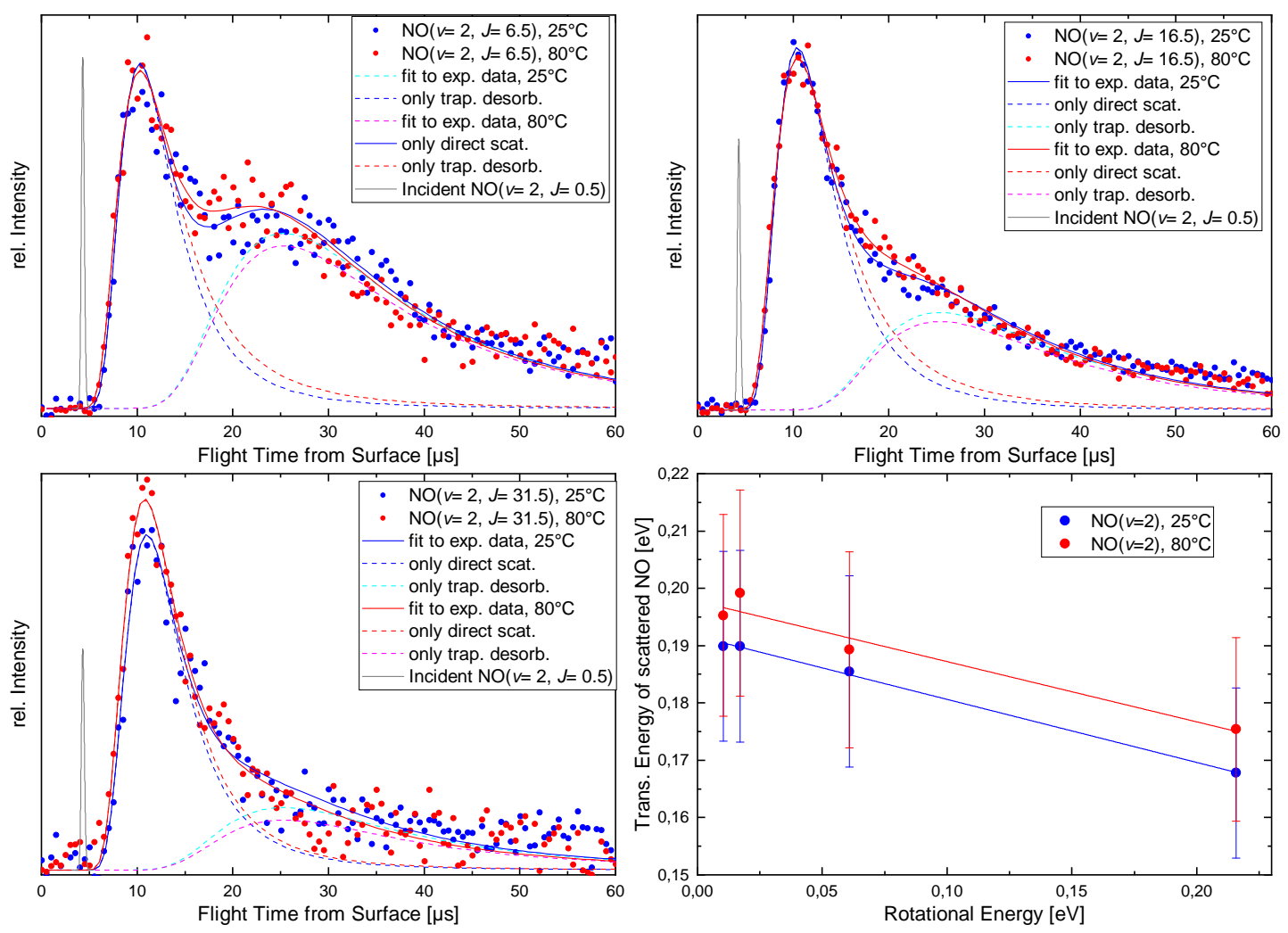

Figure 4.11: TOF distributions of $\mathrm{NO}(v=2)$ in the rotational states $J=6.5$ (top left), $J=16.5$ (top right) and $J=31.5$ (bottom left) scattered from CVD3 at surface temperatures below (blue) and above (red) the MIT temperature. In each TOF distribution of scattered NO also the TOF distribution of incident NO is shown in grey. In the bottom right panel the translational energy of the direct scattering component is plotted against the energy of the corresponding rotational states.

distribution as shown in Figure 4.11 (bottom left). For $J=6.5$ the slower component of the distribution has significantly more intensity than the fast component. This suggests that the molecules of the slower component equlibrate more with the surface resulting in more population of lower $J$ states than it is the case for directly scattered NO.

The surface temperature has no apparent influence on either distribution. Neither their position nor the relative intensity changes between room temperature and $80^{\circ} \mathrm{C}$. This can also be seen in the translational energy of the directly scattered NO plotted against rotational energy shown in the bottom right of Figure 4.11. The shown translational energy of scattered NO decreases with its rotational excitation which further supports the assumption that the measured distribution is of directly scattered $\mathrm{NO}(v=2)$. 


\section{Conclusions}

While the TOF distribution and its analysis suggests that $\mathrm{NO}(v=2)$ is partly scattered directly from CVD3 there is no indication that any aspect of it behaves differently above and below the MIT. Therefore, the TOF distribution of directly scattered NO is the same when it is scattered from $\mathrm{VO}_{2}(\mathrm{M})$ or $\mathrm{VO}_{2}(\mathrm{R})$.

In all TOF distributions an intense and broad second distribution can be seen that can be attributed to non-directly scattered NO. The intensity of this slower distribution suggests that a significant portion of the molecular beam does not scatter directly from the surface in contrast to the $\mathrm{NO}(v=11)$ scattering observed from CVD1 and CVD2. This can be caused if the surface of CVD3 is more porous than the earlier samples. If larger cavities are found on the surface NO molecules can become trapped inside and bounce several times while equilibrating with the surface.

\subsubsection{REMPI Spectra of Scattered NO}

Since the TOF distributions show a significant amount of signal which can not be attributed to direct scattering the vibrational and rotational excitation at different points of the TOF distribution are analyzed. This can give insights about the scattering mechanism of the probed molecules. For this REMPI spectra at different flight times were measured. Figure 4.12 shows such a REMPI spectrum. The figure compares the measured REMPI spectrum of scattered $\mathrm{NO}(v=2)$ to the background REMPI spectrum without overtone pumping (top panel). The bottom panel shows the background corrected REMPI spectrum and the fitted REMPI spectrum simulation. In the REMPI spectrum the $\gamma(0,2)$ and $\gamma(0,1)$ transition band are probed. Next to the $\gamma(0,1)$ transition band one can also see the $\gamma(1,2)$ band which was not analyzed since it bears the same information but has significantly less intensity than the $\gamma(0,2)$ band. The background spectrum is dominated mostly of the $\gamma(0,1)$ transition band because in the $\mathrm{NO}(v=0)$ molecular beam a small portion of $\mathrm{NO}$ is thermally excited to the $v=1$ state. The amount of thermal $\mathrm{NO}(v=2)$ in the molecular beam is too low to be detected in the REMPI spectrum. The background is also the major contributor to the $\mathrm{NO}(v=1)$ population in the shown REMPI spectrum. In the background corrected REMPI spectrum it becomes apparent that the $\gamma(0,1)$ transition band is almost completely subtracted during the background correction. The fitted simulation seen in the lower part of Figure 4.12 reproduces the experimental data sufficiently well to use its parameters for the analysis of rotational and vibrational state populations.

Figure 4.13 shows on the left side the relative population distributions for the two probed vibrational states at different flight times. The spectra for each surface temperature of 


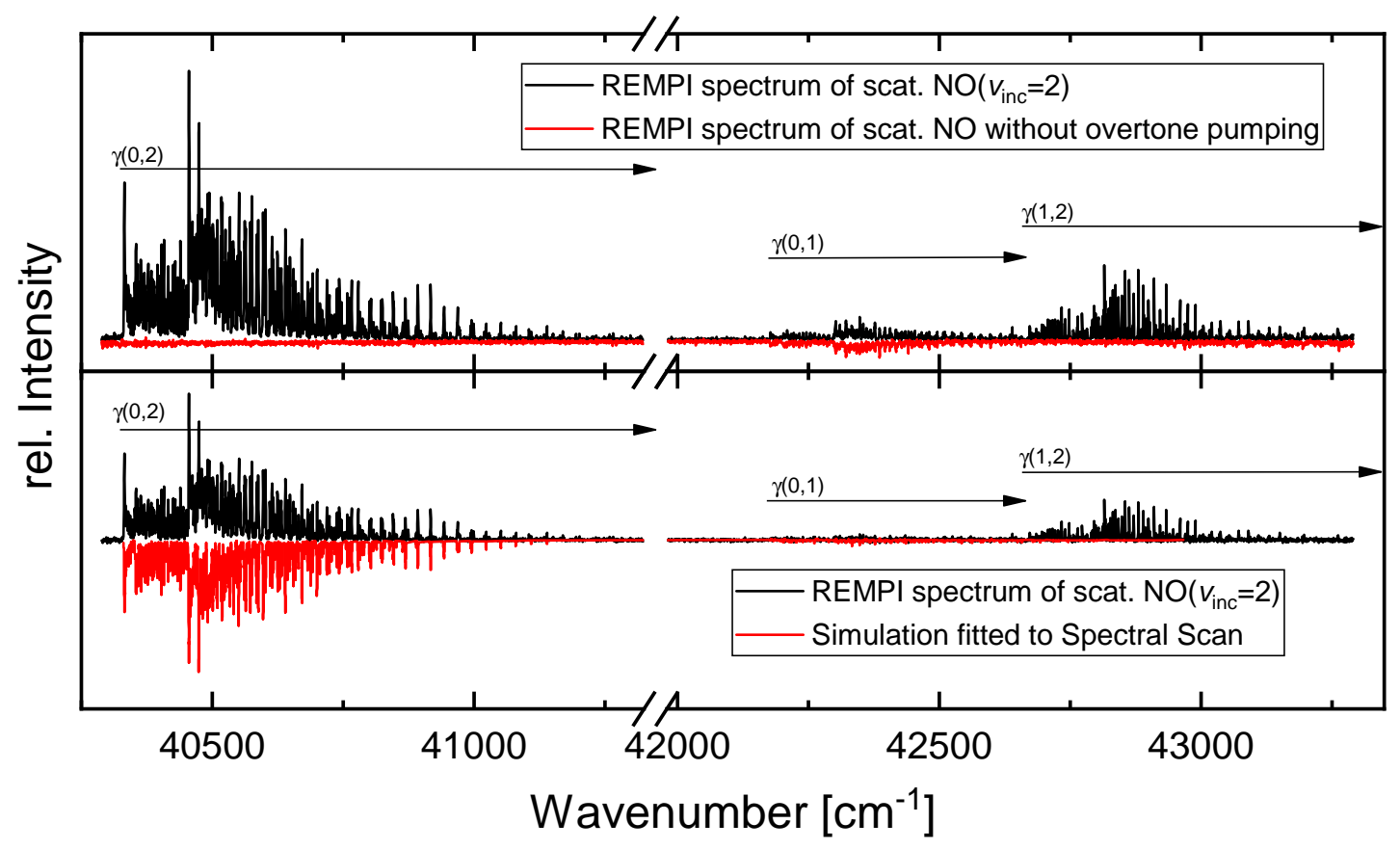

Figure 4.12: The upper plot shows the REMPI spectrum of $\mathrm{NO}\left(v_{\text {inc }}=2\right)$ scattered from CVD3 (black) with the background REMPI spectrum of NO without the overtone pumping (red). The lower plot shows the background corrected REMPI spectrum (black) and the corresponding simulation fitted to the experimental data (red). The $\gamma(1,2)$ transition band of NO is not seen in the fitted simulation because it is deliberately excluded from it. Both spectra e measured at an early flight times of $t=11 \mu$ s after the collision.

$25^{\circ} \mathrm{C}$ and $80^{\circ} \mathrm{C}$ are individually analyzed and are shown as blue and orange bars. It can be seen that at a flight time of $11 \mu \mathrm{s}$ about $95 \%$ of the molecules remain in the initial vibrational state $v=2$. For all other probed flight times only about $75 \%$ remain in the initial state. Furthermore, only for a flight time of $11 \mu$ s the population in $v=2$ is $1.6 \%$ higher for the surface below the MIT temperature than for the surface above the MIT temperature. For later probed flight times it is the other way around. This might be an indication for enhanced vibrational relaxation of $\mathrm{NO}(v=2)$ when scattered from $\mathrm{VO}_{2}(\mathrm{R})$ compared to $\mathrm{VO}_{2}(\mathrm{M})$. On the right side of the same figure vibrational state distributions for thermal NO are shown at $80^{\circ} \mathrm{C}$ and $750{ }^{\circ} \mathrm{C}$ for comparison.

Figure 4.14 shows the rotational temperatures of the $\gamma(0,2)$ transition band for different arrival times together with the TOF distribution for molecules scattered back in $\mathrm{NO}(v=2, J=16.5)$ probed via the $\mathrm{R}_{11}$ transition. The rotational temperatures measured at short flight times around the rising flank and peak of the distribution associated with direct scattering are with values of $850 \mathrm{~K}$ to $1000 \mathrm{~K}$ significantly higher than the rotational temperatures measured at the tail of the TOF distribution. Here, rotational 

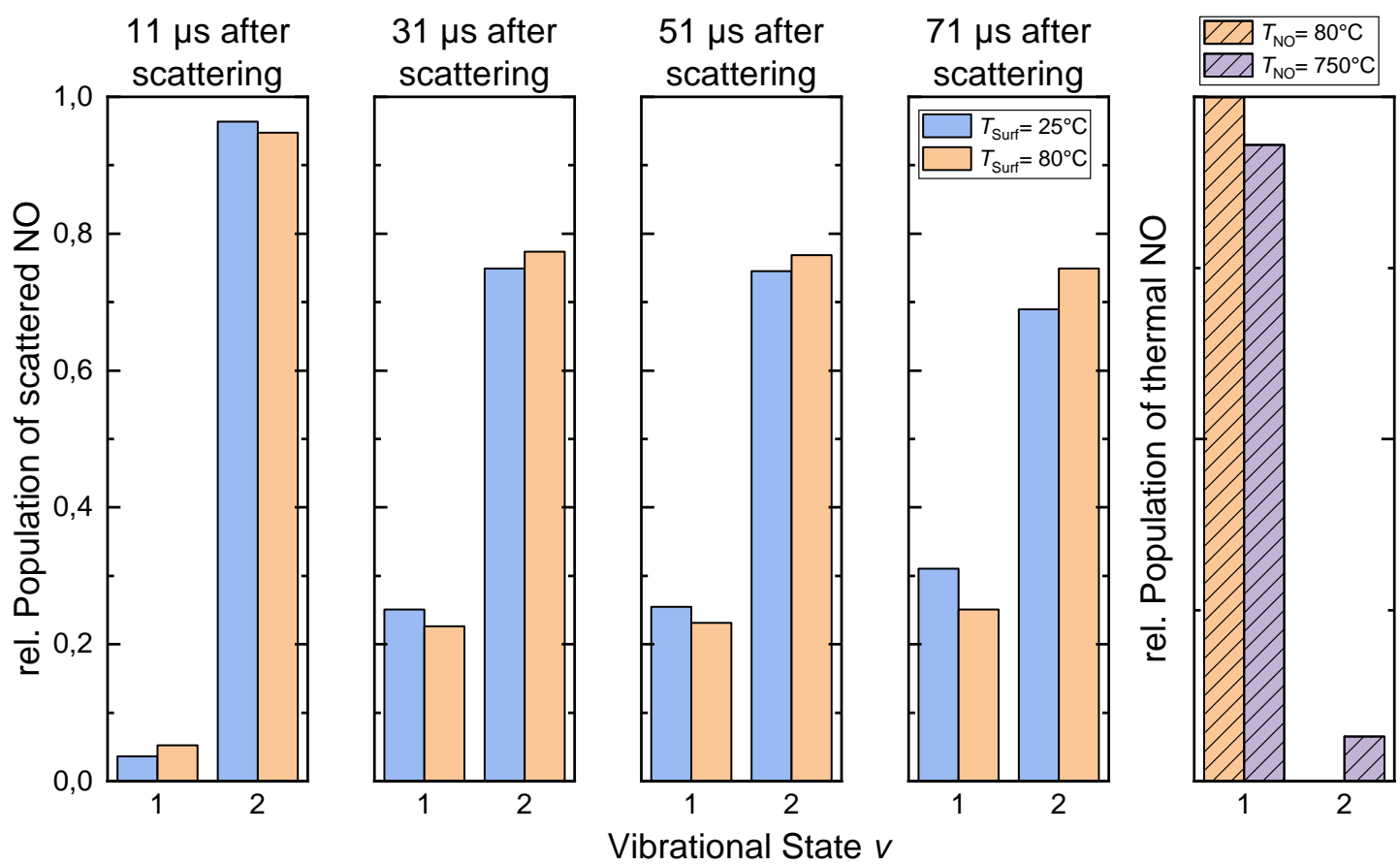

Figure 4.13: Relative population of scattered $\mathrm{NO}(v=1)$ and $\mathrm{NO}(v=2)$. $\mathrm{NO}$ is scattered from CVD3 and each plot on the left side shows a different flight time between 11 and 71 s. The single bars are discerned between surface temperatures of 25 and $80^{\circ} \mathrm{C}$. On the right side the relative population distribution of the same vibrational states in thermally excited $\mathrm{NO}{ }^{72}$ is shown. It shows the thermal relative vibrational excitation at $80^{\circ} \mathrm{C}$ and $750{ }^{\circ} \mathrm{C}$. It is apparent that the vibrational excitation of scattered $\mathrm{NO}$ is in all cases much higher than for thermal excitation at $750{ }^{\circ} \mathrm{C}$.

temperatures of $400 \mathrm{~K}$ to $700 \mathrm{~K}$ are measured. This observation contradicts that a full equilibration the NO molecules of the slow component are fully equilibrated with the surface. This might indicate that these molecules undergo some scattering mechanism which is intermediate between direct scattering and trapping desorption such that full equilibration cannot take place.

All differences between the rotational temperature of NO scattered from CVD3 above or below the MIT are within the error interval except for the rotational temperatures measured at a flight time of $51 \mu \mathrm{s}$. This value can be considered an outlier.

\section{Conclusions}

The analysis of REMPI spectra of NO scattered from CVD3 shows that the two components of the TOF distributions stem from two separate scattering channels. The component at short flight times one can be identified as direct scattering with its high 


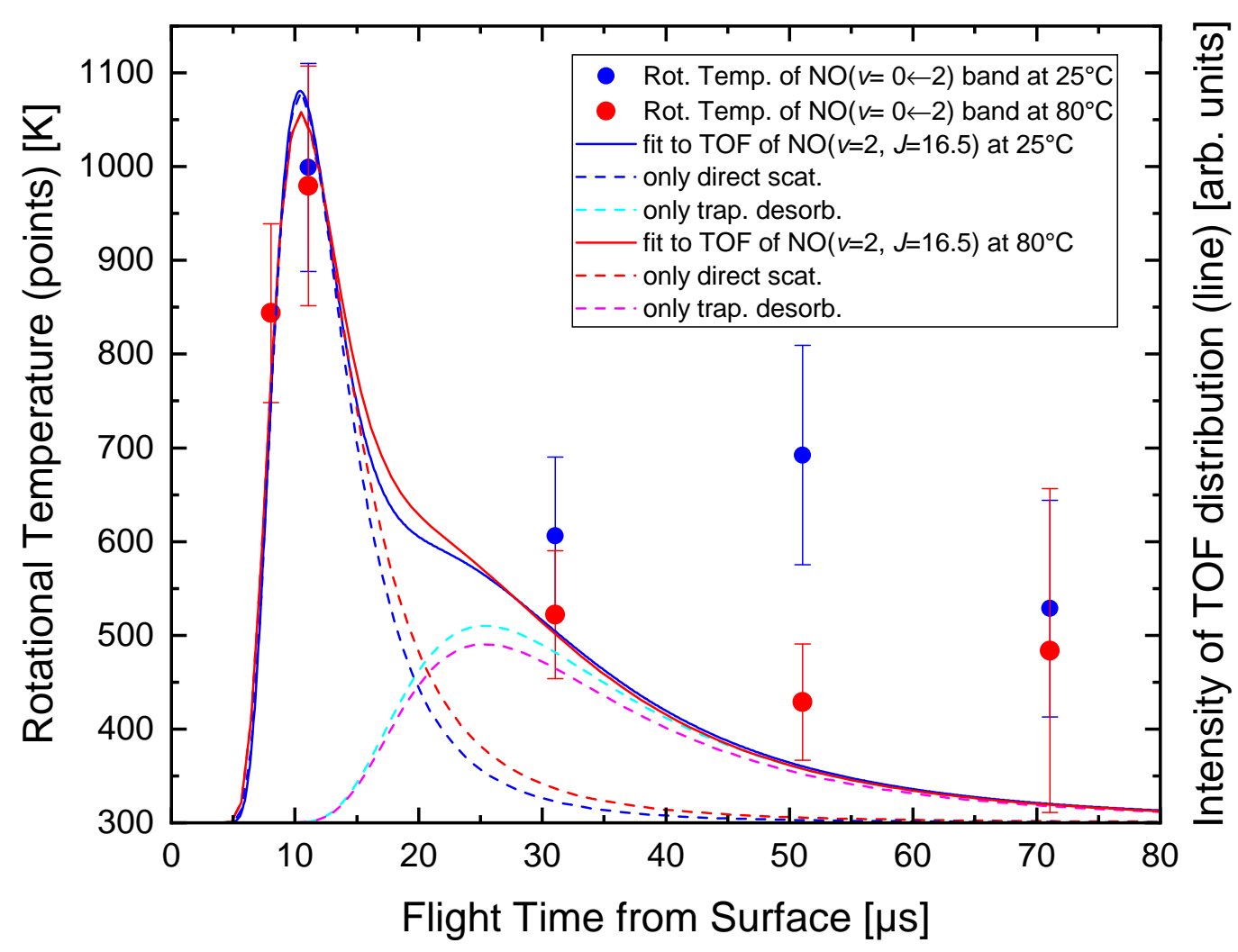

Figure 4.14: Rotational temperature of the $\gamma(0,2)$ transition band of scattered NO plotted along the TOF distribution of scattered NO. The TOF distribution consists of two parts depicted by dashed lines. The early narrow distribution is a direct scattering distribution and the late broad distribution is a trapping desorption distribution. Their some is depicted by the solid line.

Table 4.5: Rotational temperatures $T_{\text {rot }}$ of the $\gamma(0,2)$ transition band of NO scattered from CVD3.

\begin{tabular}{ccccccc}
\hline TOF $[\mu \mathrm{s}]$ & & 8 & 11 & 31 & 51 & 71 \\
$T_{\text {rot }}[\mathrm{K}]$ & $T_{\text {surf }}=25^{\circ} \mathrm{C}$ & - & $1000 \pm 120$ & $600 \pm 90$ & $690 \pm 120$ & $530 \pm 120$ \\
$T_{\text {rot }}[\mathrm{K}]$ & $T_{\text {surf }}=80^{\circ} \mathrm{C}$ & $840 \pm 100$ & $980 \pm 130$ & $522 \pm 70$ & $430 \pm 70$ & $480 \pm 180$ \\
\hline
\end{tabular}


conservation of initial vibrational population and its high rotational excitation upon collision. The component at later flight times is probably generated by a several bounces on the rough surface. It is characterized by an increased loss of vibrational energy upon collision and lower rotational excitation that both still exceed the thermal excitation of the surface.

While a small decrease between the vibrational population of the initial vibrational state $v=2$ between the room temperature and the $80^{\circ} \mathrm{C}$ hot surface can be seen in Figure 4.13 both the amount of vibrational relaxation and the difference between the two phases above and below the MIT are less than is expected. The fact that in all three vibrational distributions the hot surface generates more population of $\mathrm{NO}(v=2)$ than the cold surface suggests that the vibration equilibrates to some amount with the surface. That it does not equilibrate fully with the surface is shown on the right side of Figure 4.13 . Here, the vibrational excitation of thermal NO molecules is shown for temperatures of $80^{\circ} \mathrm{C}$ and $750^{\circ} \mathrm{C}$. It can be seen that the vibrational excitation in all probed vibrational population distributions of scattered $\mathrm{NO}$ is much larger than the thermal excitaion at $750{ }^{\circ} \mathrm{C}$. For $80^{\circ} \mathrm{C}$ the population of $\mathrm{NO}(v=2)$ is negligibly small. In scattered $\mathrm{NO}, v=2$ is the vibrational state with the most population. Therefore, the tail component of the TOF distribution is unlikely to be generated by trapping desorption. It is most probably generated by NO bouncing multiple times on the surface or inside gaps and cracks of the $\mathrm{VO}_{2}$ film.

The measured rotational temperatures at early arrival times ( $8 \mu \mathrm{s}$ and $11 \mu \mathrm{s})$ are several $100 \mathrm{~K}$ hotter than the rotational temperatures at later arrival times ( $\geq 31 \mu \mathrm{s})$. This means that in the case of the directly scattered NO a large amount of translational energy is transferred to rotational quanta and little to no rotational excitation is lost due to equilibration with the surface temperature. The rotational temperatures found in the slow scattered NO are furthermore also about $100 \mathrm{~K}$ to $400 \mathrm{~K}$ higher than the surface temperature. Also there is no dependence between the surface temperature and the measured rotational temperature with all measurements at room temperature resulting in higher rotational temperatures than the measurements at $80^{\circ} \mathrm{C}$. This indicates that the slow distribution is not caused by trapping since the interaction time would be enough to result in complete thermal equilibration between the rotational quanta and the surface temperature. It is more reasonable to assume that this component is caused by NO molecules bouncing two or more times on or beneath the porous surface before they leave again. 


\subsection{Discussion}

With the NO scattering experiments from CVD1, CVD2 and CVD3 the feasibility of direct scattering from $\mathrm{VO}_{2}$ is tested. $\mathrm{NO}$ molecules show direct scattering from all CVD prepared samples but for example when scattering from CVD1 the direct scattering component can not be easily separated from other components. The ratio between direct and non-direct scattering components shown in the TOF distributions is for higher $J$ states higher than for lower $J$ states. Also the translational energy of the molecular beam plays an important role to which extend direct scattering can be observed separated from rival scattering channels.

From the CVD1 experiments it was shown that it is absolutely necessary to use $\mathrm{VO}_{2}$ films with high structural homogeneity and no macroscopic defects. While CVD2 and CVD3 appear to be less flat than CVD1 and are also more sensitive to scratches it was not possible to observe resolvable direct scattering components in the TOF distributions of low $J$ state NO scattered from CVD1. The presence of overlapping non-direct scattering components in the TOF distribution of NO scattered from CVD1 results in erroneous translational energy analysis.

The fact that the direct scattering channel is dominant when scattering NO from CVD1 and CVD2 could be shown by the rotational temperature analysis of the REMPI spectra. For both surfaces rotational temperatures several $100 \mathrm{~K}$ above the surface temperature were measured. The measured rotational temperatures also show a linear dependence on the translational energy as shown in Figure 4.9. Therefore, a significant portion of the translational energy of the incident molecular beam is transformed to rotational excitation and the rotational excitation of the molecules also does not equlibrate with the surface degrees of freedom.

While the TOF distribution of both CVD2 and CVD3 showed another component of slower non-directly scattered NO, both components are clearly separated and can be probed individually. This is shown by the REMPI spectra measured along the TOF distribution from CVD3 (Figure 4.13 and 4.14). Depending on the flight time different rotational temperatures and vibrational state distributions were measured. For flight times corresponding to the fast component the measured rotational temperatures are far higher while the vibrational relaxation is significantly reduced compared to REMPI spectra at flight times corresponding to the slow component. Both the vibrational relaxation at the fast component and at the slow component show a small dependence on the surface temperature though in different direction. For the fast component about $1.5 \%$ more $\mathrm{NO}$ is found in $v=2$ than scattered from $\mathrm{VO}_{2}(\mathrm{M})$ compared to $\mathrm{NO}(v=2)$ scattering from $\mathrm{VO}_{2}(\mathrm{R})$. This can be seen as a first indication for an increased relaxation 
probability of $\mathrm{NO}$ scattered from $\mathrm{VO}_{2}(\mathrm{R})$ compared to $\mathrm{VO}_{2}(\mathrm{M})$. Although, the observed vibrational relaxation probability of $\mathrm{VO}_{2}(\mathrm{M})$ and $\mathrm{VO}_{2}(\mathrm{R})$ is significantly lower than what is expected for a metal with their respective work function 15 .

For CVD1 and CVD2 no temperature dependence on the intensity of the observed vibrational transition bands can be observed. This can be caused by the fact that after the scattering experiments neither showed a resistance dependence on the MIT and large amounts of carbon contamination are found on the surface. While CVD3 is also sputtered with $\mathrm{Ar}^{+}$-ions and most carbon could be removed a small amount is still detectable using AES. The low vibrational relaxation probability can be explained by the fact that the film of CVD3 is not pure $\mathrm{VO}_{2}$ but a compound with carbon. Also a MIT contrast of 220 is measured on CVD3. The resistance change of only two orders of magnitude might be too low to see an intense effect on the vibrational relaxation probability.

The exploratory work with CVD prepared surfaces convinced us to concentrated on experiments using surfaces with better smoothness and higher MIT contrast. These surfaces are found in RFMS prepared thin films discussed in chapter 6 . 


\section{Scattering of $\mathrm{NO}(v=11)$ from Silicon Dioxide}

The experiments with CVD prepared $\mathrm{VO}_{2}$ showed that $\mathrm{NO}$ scattered from $\mathrm{VO}_{2}$ exhibits little vibrational relaxation. This behavior is shown for both the low electrically conducting $\mathrm{VO}_{2}(\mathrm{M})$ and the high electrically conducting $\mathrm{VO}_{2}(\mathrm{R})$ and was formally only observed for NO scattered from insulator material ${ }^{958}$. To obtain reference data of insulator scattering that is comparable to the $\mathrm{VO}_{2}$ scattering shown in this work, $\mathrm{NO}$ scattering experiments were conducted with a reference insulator surface.

\subsection{Sample Characterization}

The insulator surface used in the NO scattering experiments described in this chapter consists of a $\mathrm{SiO}_{2}$ substrate similar to those used for the deposition of CVD1 and CVD2. While the $\mathrm{SiO}_{2}$ is clear and colorless it is not a single crystal with a distinct surface orientation. In consequence, the sample has probably a rough structure which makes it better comparable to the likewise rough $\mathrm{VO}_{2}$ samples.

The insulator properties of $\mathrm{SiO}_{2}$ make AES measurements impossible. Since $\mathrm{SiO}_{2}$ does not conduct electricity the charge build-up from the accelerated electrons can not be discharged fast enough. In consequence, it was not possible to monitor the elemental composition of the surface during the experiments. Nevertheless, it is safe to assume that the surface was initially contaminated with significant amounts of carbon. Therefore, for surface preparation the $\mathrm{SiO}_{2}$ sample was sputtered for 20 min with $\mathrm{O}_{2}{ }^{+}$-ions accelerated to $1 \mathrm{kV}$ at the beginning of each day of measurement. It is assumed that charge build-up during sputtering is less of a problem because $\mathrm{Ar}^{+}$-ions have a much higher momentum of inertia than electrons. In consequence, they are less likely to be reflected from charges on the surface. The $\mathrm{SiO}_{2}$ surface was kept at room temperature at all times. 


\subsection{Angular Distribution of Scattered NO}

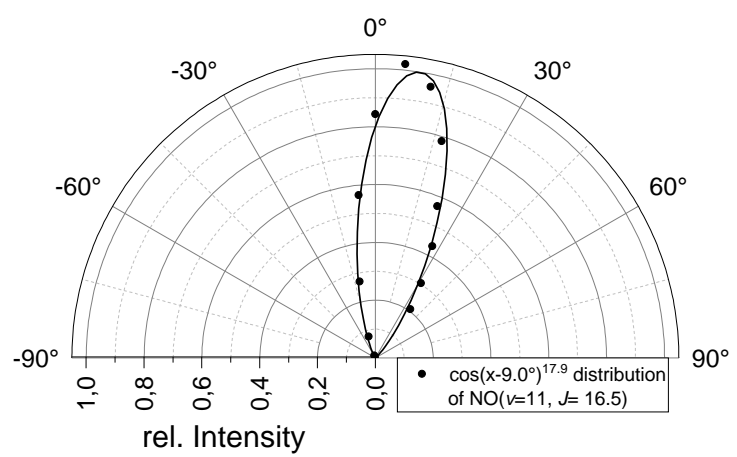

Figure 5.1: Angular distribution of scattered $\mathrm{NO}(v=11, J=16.5)$ from $\mathrm{SiO}_{2}$ and detected via the $\mathrm{Q}_{11}(16.5)$ line of the $\gamma(4,11)$ band.

Figure 5.1 shows the angular distribution of $\mathrm{NO}(v=11, J=16.5)$ scattered from $\mathrm{SiO}_{2}$. The used transition is the $\mathrm{Q}_{11}(16.5)$ line of the $\gamma(4,11)$ transition band. The distribution follows a $\cos ^{17.9}$ function and is therefore narrow enough that it can be considered to be generated by direct scattering. The angular offset is caused by the tilt of the surface mount in respect to the incident molecular beam.

\subsection{Time-of-Flight Spectroscopy of Scattered NO}

The left side of Figure 5.2 shows the results from the translational energy analysis of TOF distributions measured at the wavelengths of the $\mathrm{R}_{22}(5.5), \mathrm{Q}_{11}(9.5), \mathrm{Q}_{11}(16.5)$ and $\mathrm{Q}_{22}(30.5)$ transition lines of the $\gamma(4,11)$ band of NO. The shown data points are listed in Table 5.1. Incident molecular beam pulses with translational energies of $0.88 \mathrm{eV}$, $0.76 \mathrm{eV}, 0.65 \mathrm{eV}$ and $0.55 \mathrm{eV}$ are used. The points are fitted with linear progressions which all show a negative slope. This is characteristic behavior for directly scattered NO molecules where the translation energy of scattered NO decreases with rotational excitation.

The for rotational excitation adjusted translational energies of scattered NO are plotted against the corresponding translational energies of incident NO. The resulting Baule plot is shown on the right side of Figure 5.2. The slope of the linear progression fitted to the data points corresponds to an effective surface mass of $m=128 \mathrm{amu}$. This value is significantly higher than the masses of $\mathrm{Si}(m(\mathrm{Si})=28 \mathrm{amu})$ and $\mathrm{O}(m(\mathrm{O})=16 \mathrm{amu})$. It is caused by the rigid crystal structure of $\mathrm{SiO}_{2}$ where the individual particles are strongly bound to each other. 

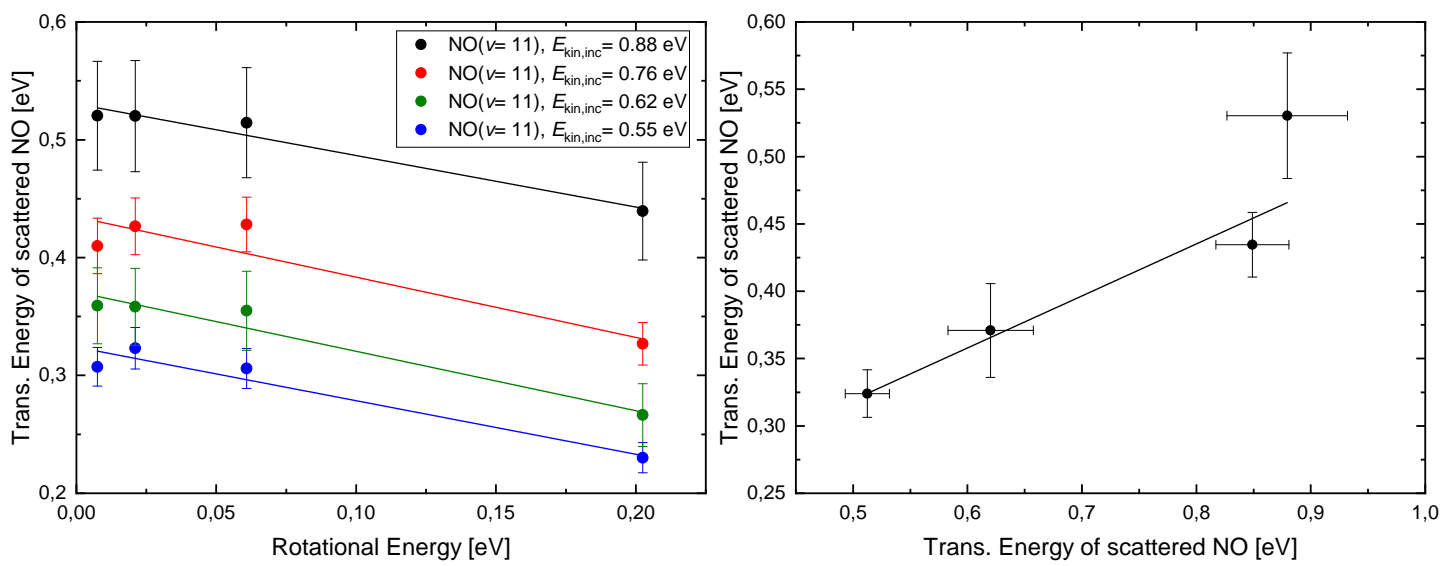

Figure 5.2: Left side: Plot of translational energy of scattered $\mathrm{NO}(v=11)$ against rotational energy of the respective probed rotational line. Scattering occurred from a $\mathrm{SiO}_{2}$ substrate and with incident molecular beam of different translational energies $E_{\text {trans,inc }}$. Linear fits are added to extrapolate the translational energy of scattered NO to $J=0$.

Right side: Baule plot of for rotational excitation adjusted translational energy of NO scattered from $\mathrm{SiO}_{2}$ against the corresponding translational energy of incident NO. The slope of the linear progression fitted to the data points corresponds to an effective surface mass of $m=128 \mathrm{amu}$.

Table 5.1: Mean translational energy from TOF distribution analysis of $\mathrm{NO}(v=11)$ scattered from $\mathrm{SiO}_{2}$.

\begin{tabular}{ccccc}
\hline Rovibrational State & $\begin{array}{c}\langle E\rangle_{\text {trans }} \\
\text { in meV }\end{array}$ & $\begin{array}{c}\langle E\rangle_{\text {trans }} \\
\text { in meV }\end{array}$ & $\begin{array}{c}\langle E\rangle_{\text {trans }} \\
\text { in meV }\end{array}$ & $\begin{array}{c}\langle E\rangle_{\text {trans }} \\
\text { in meV }\end{array}$ \\
\hline Incident $\mathrm{NO}(v=11, J=0.5)$ & $880 \pm 60$ & $760 \pm 40$ & $620 \pm 40$ & $510 \pm 20$ \\
$(v=11, J=5.5)$ & $520 \pm 50$ & $410 \pm 30$ & $359 \pm 40$ & $307 \pm 20$ \\
$(v=11, J=9.5)$ & $520 \pm 50$ & $427 \pm 30$ & $358 \pm 40$ & $323 \pm 20$ \\
$(v=11, J=16.5)$ & $514 \pm 50$ & $428 \pm 30$ & $355 \pm 40$ & $306 \pm 20$ \\
$(v=11, J=30.5)$ & $440 \pm 50$ & $327 \pm 20$ & $266 \pm 30$ & $230 \pm 20$ \\
\hline
\end{tabular}




\subsection{REMPI Spectra of Scattered NO}

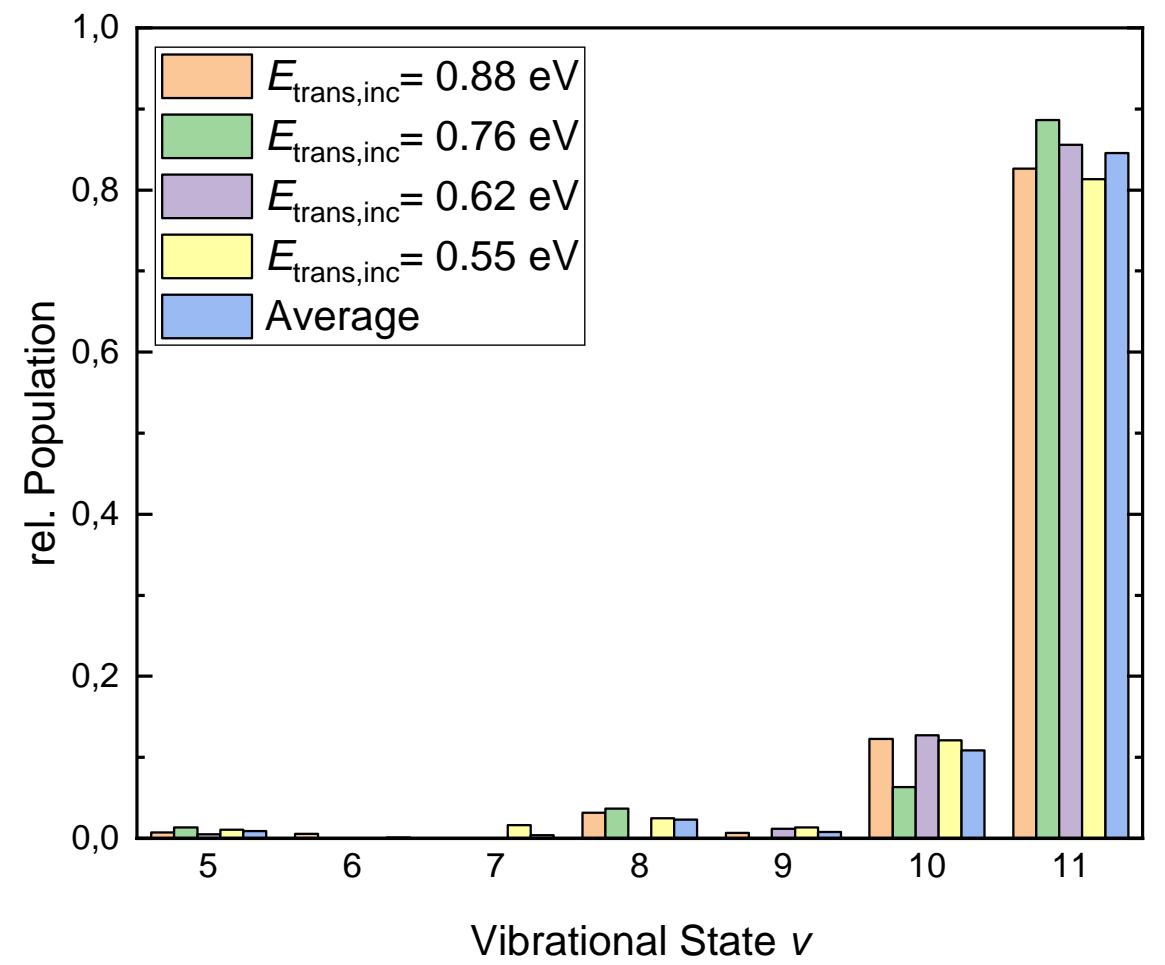

Figure 5.3: Vibrational population distribution of $\mathrm{NO}(v=11)$ scattered from $\mathrm{SiO}_{2}$ with different translational energies of the incedent molecular beam pulse. The blue bars show the average of the other bars.

Figure 5.3 shows vibrational population distributions of $\mathrm{NO}(v=11)$ scattered from $\mathrm{SiO}_{2}$ for incident molecular beam pulses with different translational energies. In the shown vibrational population distributions $80 \%$ to $90 \%$ of the total population is found in $v=11$. About $5 \%$ to $12 \%$ of the total population is found in $v=10$. The other vibrational states $v<10$ have little to no population. The population in these vibrational states can mostly be traced to noise in the REMPI signal or is generated by the background correction. The highest vibrational population of these lower vibrational states can be found in $v=8$. Only for a translational energy of $E_{\text {trans,inc }}=0.62 \mathrm{eV}$ it is found to be zero. The reason for this can be found in the REMPI spectrum shown in Figure 5.4. The observed $\gamma(2,8)$ transition band is located close to the high $J$ state transition lines of the $\gamma(4,11)$ transition band. The algorithm used to fit the experimental REMPI spectrum with a simulated REMPI spectrum counts some of the line intensity of the $\gamma(4,11)$ transition band to the $\gamma(2,8)$ transition band. This leads to exaggerated populations of $\mathrm{NO}(v=8)$. 


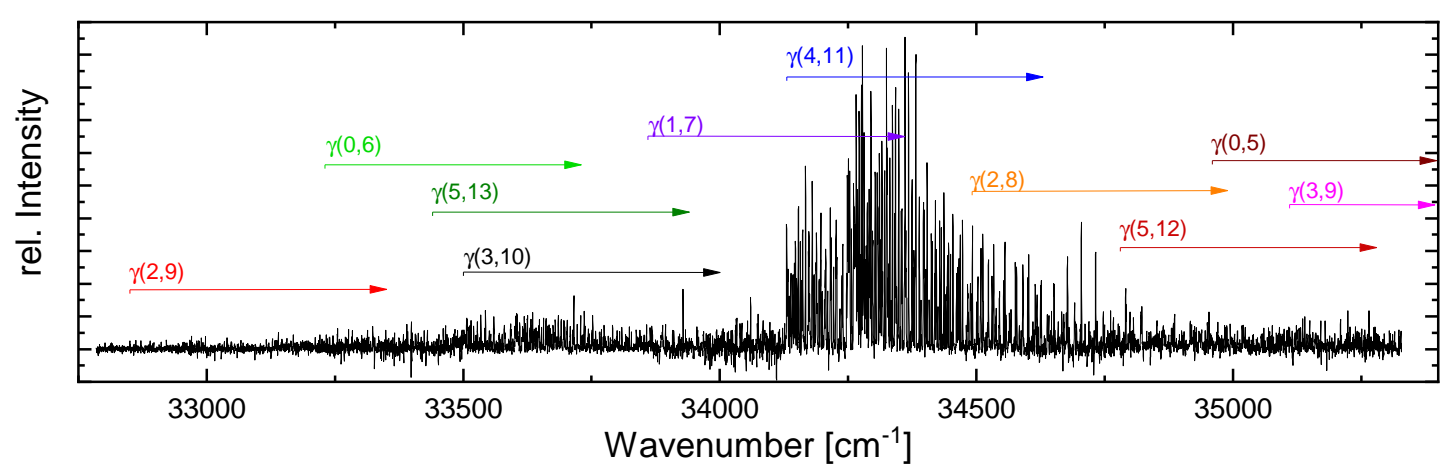

Figure 5.4: Exemplary background corrected REMPI spectrum of $\mathrm{NO}(v=11)$ scattered from $\mathrm{SiO}_{2}$. Potentially observable transition bands are marked with arrows.

Table 5.2: Mean rotational energies $\langle E\rangle_{\text {rot }}$ and temperatures $T_{\text {rot }}$ from REMPI spectra of NO scattered from $\mathrm{SiO}_{2}$ with different translational energies $E_{\text {trans,inc }}$ in the incident molecular beam.

\begin{tabular}{ccccc}
\hline $\begin{array}{c}E_{\text {trans,inc }} \\
\text { in meV }\end{array}$ & $\begin{array}{c}\langle E\rangle_{\text {rot }}(v=10) \\
\text { in meV }\end{array}$ & $\begin{array}{c}T_{\text {rot }}(v=10) \\
\text { in } \mathrm{K}\end{array}$ & $\begin{array}{c}\langle E\rangle_{\text {rot }}(v=11) \\
\text { in } \mathrm{meV}\end{array}$ & $\begin{array}{c}T_{\text {rot }}(v=11) \\
\text { in } \mathrm{K}\end{array}$ \\
\hline $879 \pm 60$ & 56 & $960 \pm 250$ & 46 & $720 \pm 50$ \\
$762 \pm 70$ & 61 & $930 \pm 650$ & 48 & $730 \pm 80$ \\
$620 \pm 40$ & 42 & $760 \pm 160$ & 39 & $630 \pm 50$ \\
$552 \pm 90$ & 47 & $680 \pm 180$ & 34 & $500 \pm 30$ \\
\hline
\end{tabular}

The REMPI spectra are analyzed for the rotational excitation of the observed vibrational states. The results are listed in Table 5.2. Figure 5.5 shows the data points plotted against the translational energy of the incident molecular beam pulse. Both for the $\gamma(3,10)$ and the $\gamma(4,11)$ transition band a linear dependence between the rotational temperature and the translational energy of the incident molecular beam can be observed. The values for $v=10$ are about $10 \%$ higher than the corresponding values for $v=11$. The difference between the rotational temperature of $\operatorname{NO}(v=11)$ and $\mathrm{NO}(v=10)$ are just within the error of the data points. The large error of the rotational temperature of $\mathrm{NO}(v=10)$ is caused by the low intensity of the probed $\gamma(3,10)$ band. The mean rotational energy of $\mathrm{NO}(v=11)$ mirrors the rotational temperature of $\mathrm{NO}(v=11)$. The mean rotational energy of $\mathrm{NO}(v=10)$ shows high fluctuations along the translational energy of the incident molecular beam pulse. This is also caused by the low intensity of the $\gamma(3,10)$ transition band which leads to values with high uncertainty. 

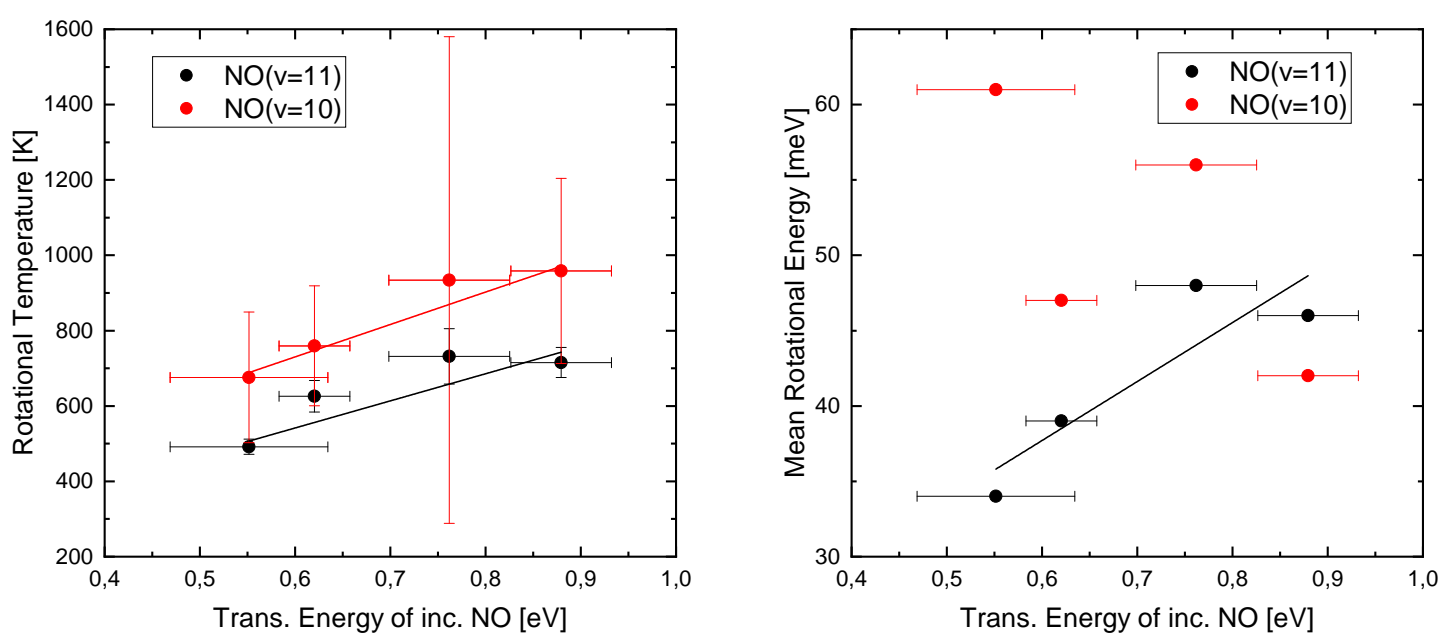

Figure 5.5: Rotational temperature (left side) and mean rotational energy (right side) of the $\gamma(4,11)$ and $\gamma(3,10)$ transition band of $\mathrm{NO}(v=11)$ scattered from $\mathrm{SiO}_{2}$. Linear progressions are added to the data points to illustrate the linear dependence between rotational excitation and translational energy of incident NO. Because of the large measurement uncertainty of the mean rotational energy of $\mathrm{NO}(v=10)$ no linear dependence can be seen in this case. No error was assigned to the mean rotational energies because only one spectrum for each incident molecular beam energy was measured.

\subsection{Discussion}

The results of the scattering experiments of $\mathrm{NO}(v=11)$ from $\mathrm{SiO}_{2}$ show clearly that NO scatters through the direct scattering channel.

NO scatters with a narrow angular distribution from the surface. The angular offset of this distribution corresponds to the tilt of the surface in respect to the incident molecular beam. The translational energy of the scattered NO molecules descreases linearly with their rotational excitation. After scattering NO shows high rotational excitation which scales with the translational energy of the incident molecular beam pulse. All these points are strong indicators for direct scattering.

The observed rotational excitation of the relaxed $\mathrm{NO}(v=10)$ turns out higher than the rotational excitation of the $\mathrm{NO}(v=11)$ molecules that retain their initial vibrational state. In previous experiments with $\mathrm{NO}$ scattered from $\mathrm{Au}(111)^{41}$ the opposite effect is observed. Here, the rotational excitation of scattered NO decreases the more it relaxes vibrationally. On the other hand, due to the high error of the rotational temperature of $\mathrm{NO}(v=10)$ it can not be said for certain if this is a persistent effect or if it is caused by measurement uncertainty. 
The Baule plot shows that the $\mathrm{SiO}_{2}$ surface has an effective mass of $m=128 \mathrm{amu}$ caused by the rigid crystal lattice.

Furthermore, it is shown that the scattered NO retains its initial vibrational state $v=11$ with a probability of $80 \%$ to $90 \%$. Almost all the population of the relaxed NO molecules is found in $v=10$. The observed populations of $\mathrm{NO}(4<v<10)$ are so low that is can be considered to be within the measurement uncertainty. In consequence, they are assumed to be zero. Since no transition bands of $\operatorname{NO}(v=0,1,2,3,4)$ are probed with the scanned spectral region it cannot be said for certain if some relaxation of $\mathrm{NO}(v=11)$ occurs to these states. 



\section{Scattering of NO from RFMS prepared $\mathrm{VO}_{2}$ thin films}

This chapter describes the NO scattering experiments from RFMS prepared $\mathrm{VO}_{2}$ thin films. It is mainly concerned with the question if and how the MIT of $\mathrm{VO}_{2}$ influences the relaxation probability of scattered NO.

\subsection{Scattering of $\mathrm{NO}(v=3)$ from RFMS1 and RFMS2}

\subsubsection{Sample Characterization of RFMS1 and RFMS2}

For the scattering of $\mathrm{NO}(v=3)$ two RFMS prepared $\mathrm{VO}_{2}$ thin films are used, which are labeled in the following as RFMS1 and RFMS2. Pictures and surface analysis are shown in Figure 6.1 and 6.2. Both samples have a flat and reflecting surface with no visible defects and are roughly of the same size. After they were retrieved from the chamber no visible changes of the surface or its reflectivity were noticeable. Also in contrast to the CVD prepared films, no scratching marks from the 4-Probe are seen on any used RFMS prepared thin film.

The main difference between the two samples is that RFMS1 was prepared only by being sputtered twice for $5 \mathrm{~min}$ with $\mathrm{Ar}^{+}$-ions with $1 \mathrm{kV}$ acceleration before the day of the experiment. At the beginning of the day of the scattering experiments it was sputtered for 2 min with $\mathrm{Ar}^{+}$-ions with $1 \mathrm{kV}$ acceleration. RFMS2 on the other hand was sputtered 4 times for 5 min each with $\mathrm{Ar}^{+}$-ions with $1 \mathrm{kV}$ acceleration and then 3 additional times for $10 \mathrm{~min}$ each with $\mathrm{O}_{2}^{+}$-ions with $1 \mathrm{kV}$ acceleration. Figure 6.1 (a) and 6.2 (a) show that while RFMS2 is sputtered much more intensely it retained about 4 times more MIT contrast (150) than RFMS1 (MIT contrast of 40). Furthermore, RFMS2 showed afterwards no argon inclusions in the AES shown in Figure 6.2 (c). It should to be noted that RFMS2 started out with a much more intense initial MIT contrast but also more severe carbon contamination. 

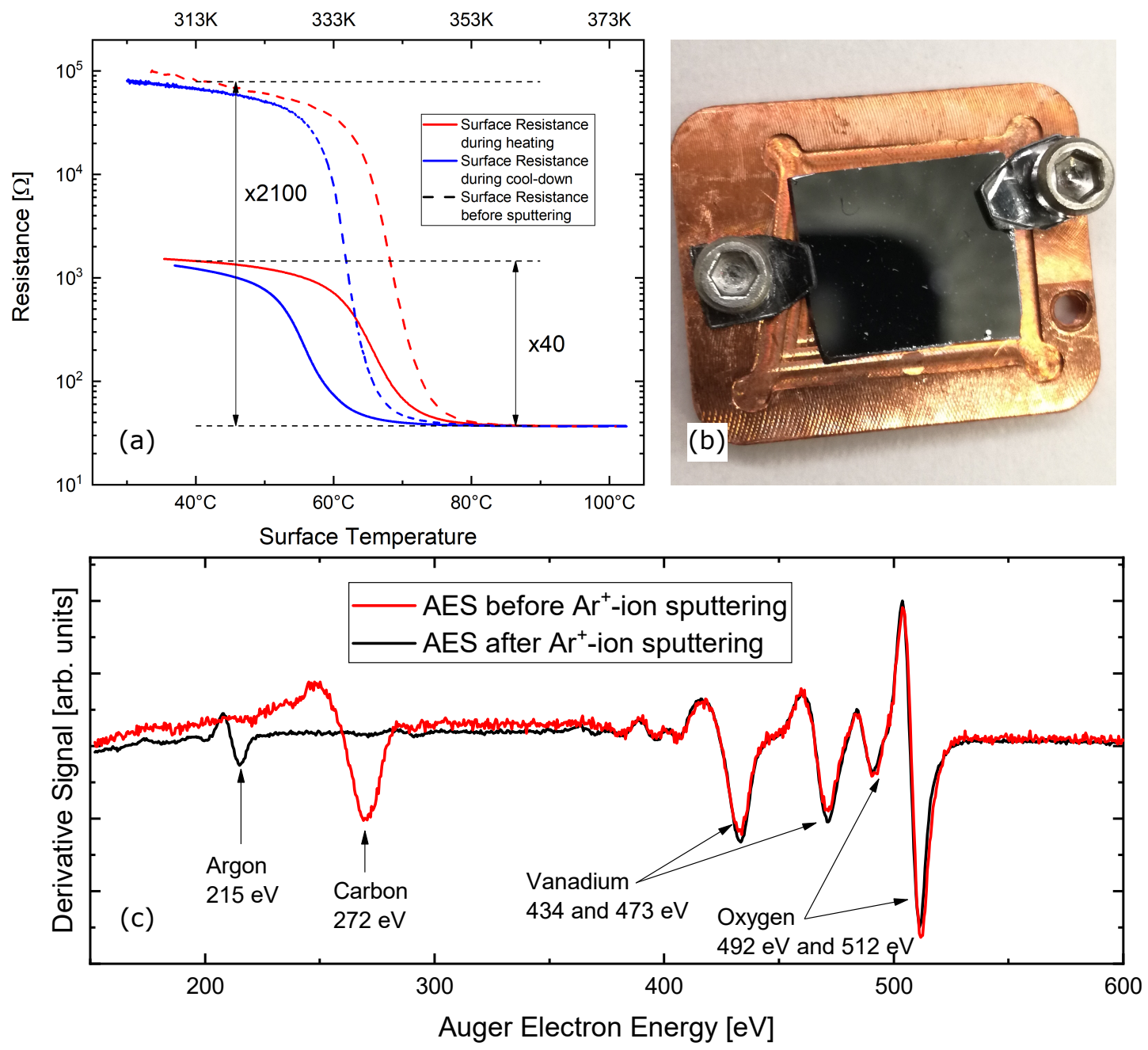

Figure 6.1: Sample characteristics of RFMS1. (a) shows the surface resistance against temperature plot of RFMS1 before and after sputtering with $1 \mathrm{kV} \mathrm{Ar}{ }^{+}$-ions. (b) shows an image of the surface mounted to the copper frame. (c) shows the AES of RFMS1 before and after sputtering. 

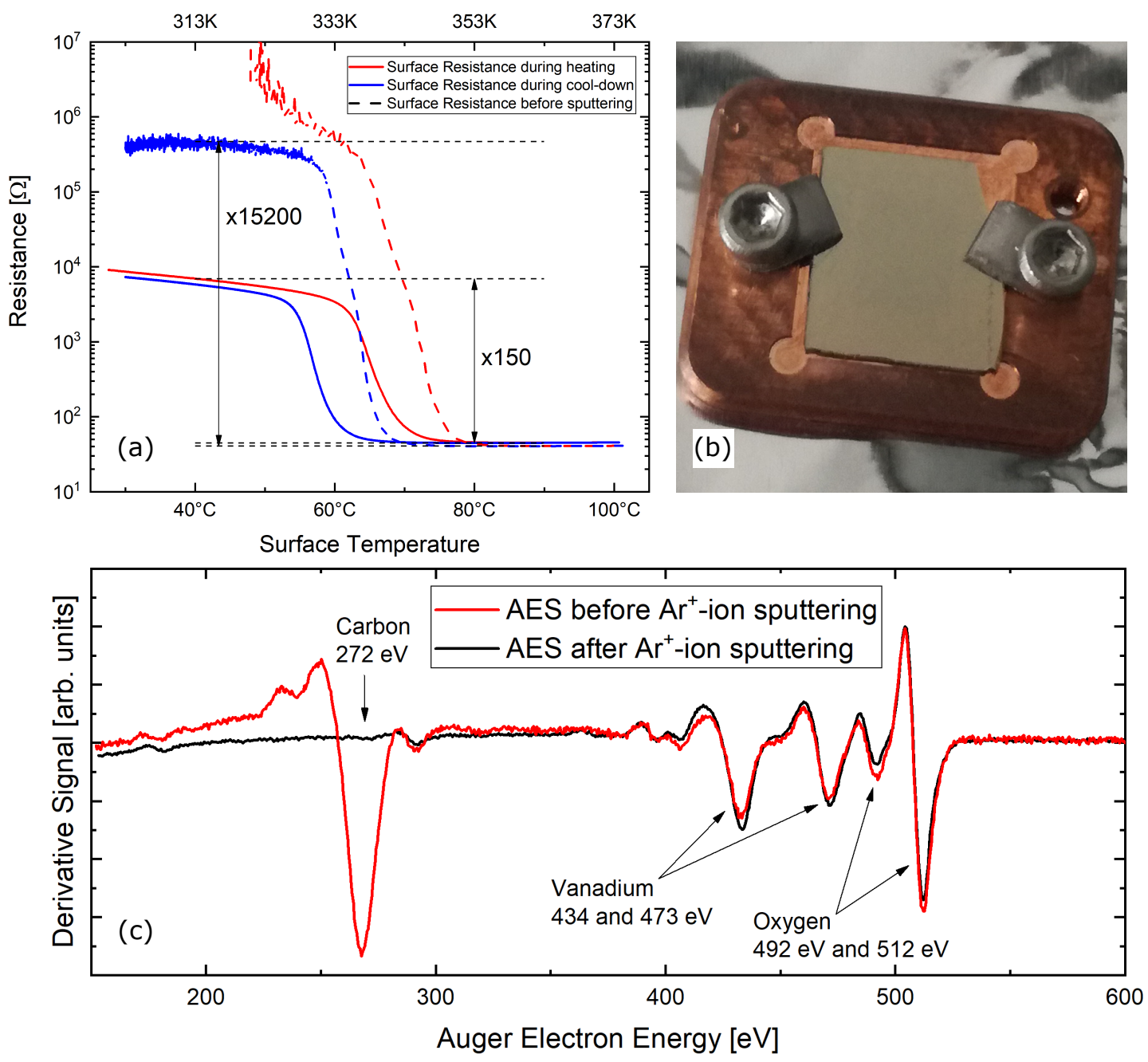

Figure 6.2: Sample characteristics of RFMS2. (a) shows the surface resistance against temperature plot of RFMS2 before and after sputtering with $1 \mathrm{kV} \mathrm{Ar}{ }^{+}$-ions and $\mathrm{O}_{2}^{+}$-ions. (b) shows an image of the surface mounted to the copper frame. (c) shows the AES of RFMS2 before and after sputtering. 


\subsubsection{Angular Distribution of Scattered NO}

Figure 6.3 shows the angular distributions of scattered $\mathrm{NO}(v=3)$. The the molecules are detected via the $\mathrm{R}_{11}(J=16.5)$ transition line of the $\gamma(1,3)$ transition band for scattering from RFMS1. For scattering from RFMS2 the $\mathrm{R}_{11}(J=6.5)$ transition line of the same band is used. The angular distributions are fitted with a $\cos ^{10.6}$ and a $\cos ^{11.6}$ function, respectively. Both scattering experiments show the, for direct scattering, typical narrow distribution. The distribution from RFMS1 has a higher angular offset of $22.3^{\circ}$ compared to the distribution from RFMS2. Here, it is only offset by $1.8^{\circ}$. These different angular offsets correspond to the surface tilting angle of the surface mount. RFMS1 was mounted in at an angle to incident molecular beam while RFMS2 was placed perpendicular to the incident molecular beam.

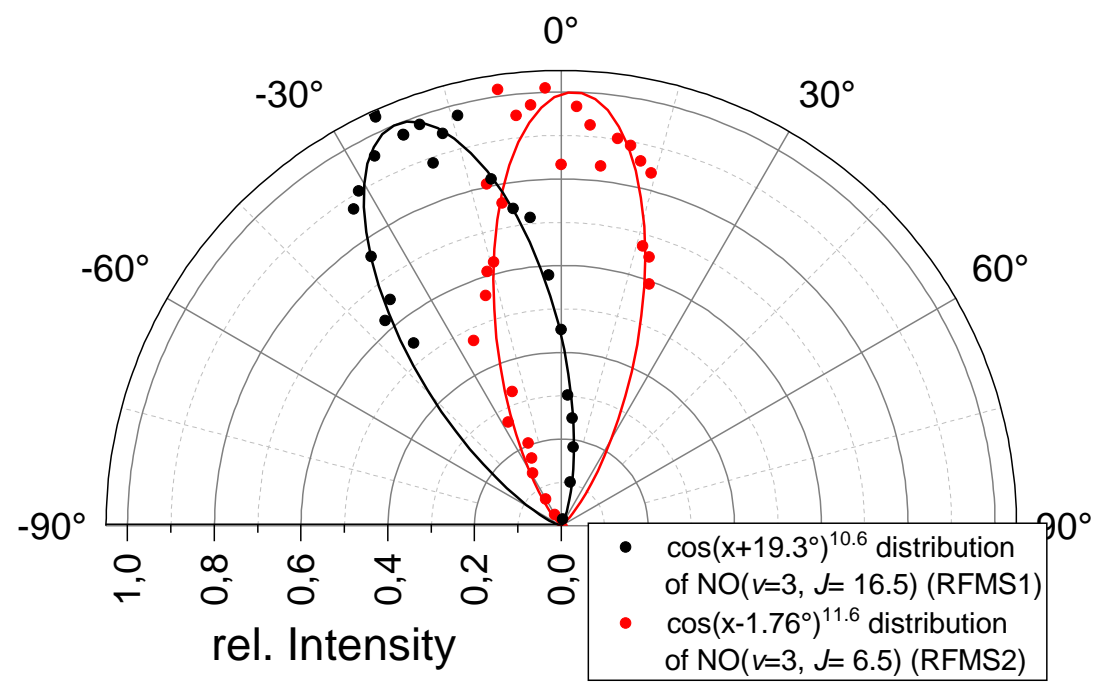

Figure 6.3: Angular distributions of $\mathrm{NO}(v=3, J=6.5)$ and $\mathrm{NO}(v=3, J=16.5)$ scattered from RFMS1 and RFMS2, respectively. The NO molecules are detected via the $\mathrm{R}_{11}$ transition line of the $\gamma(1,3)$ band in both cases. The different angular offset is caused by the fact that RMFS1 is mounted at an angle to the incident molecular beam. RFMS2 is mount perpendicular to the molecular beam.

\subsubsection{Time-of-Flight Spectroscopy of Scattered NO}

TOF distributions of NO scattered from RFMS1 are measured at the wavelengths of the $\mathrm{R}_{11}(6.5), \mathrm{R}_{11}(16.5)$ and $\mathrm{P}_{22}(30.5)$ transition lines of the $\gamma(1,3)$ band, the $\mathrm{R}_{22}(6.5)$, $\mathrm{R}_{22}(16.5)$ and $\mathrm{R}_{22}(25.5)$ transition lines of the $\gamma(0,2)$ band and the $\mathrm{R}_{22}(6.5), \mathrm{R}_{22}(16.5)$ and $\mathrm{R}_{22}(31.5)$ transition lines of the $\gamma(0,2)$ band. The TOF distributions were measured at two different surface temperatures, below $\left(25^{\circ} \mathrm{C}\right)$ and above $\left(80^{\circ} \mathrm{C}\right)$ the MIT 


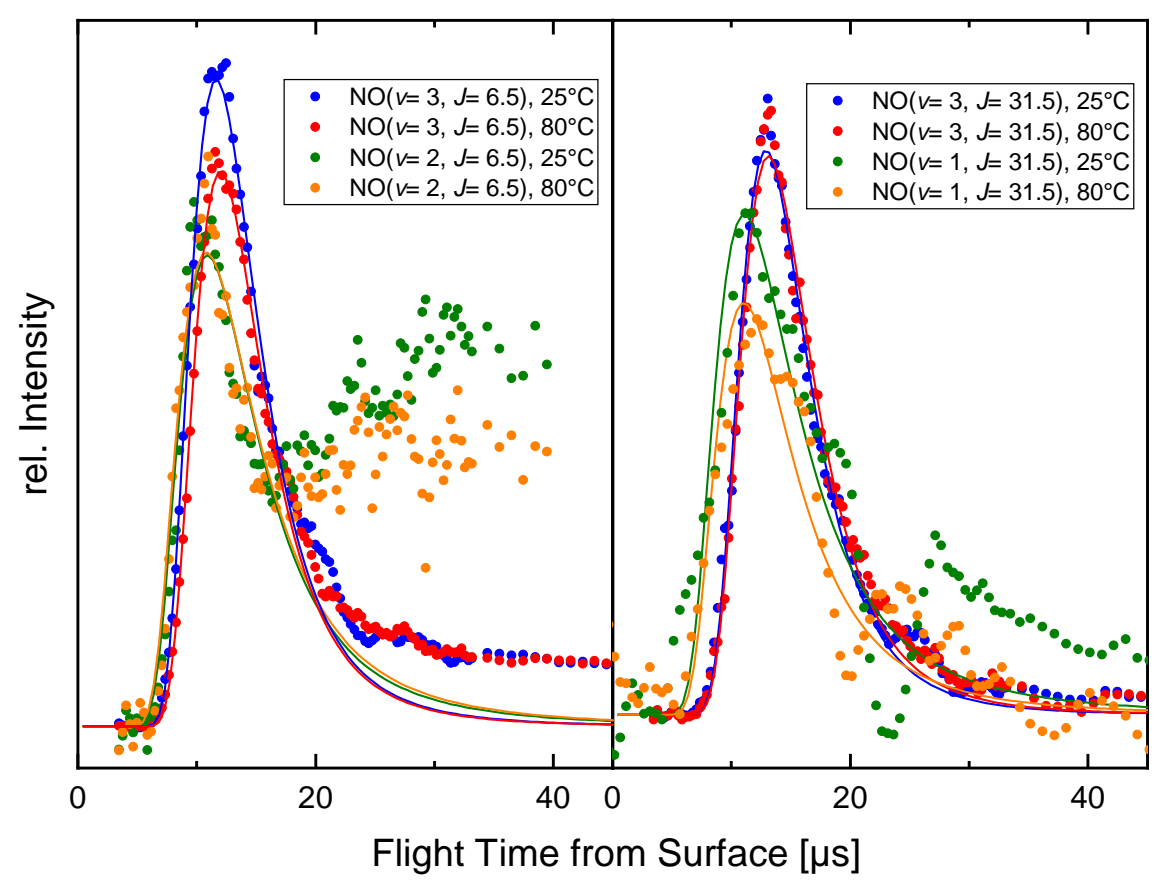

Figure 6.4: Exemplary TOF distributions of NO scattered from RFMS1. The left side shows TOF distributions of $\mathrm{NO}(v=3, J=6.5)$ (blue, red) and $\mathrm{NO}(v=2, J=6.5$ ) (green, orange) scattered from RFMS1 with a surface temperature of $25^{\circ} \mathrm{C}$ (blue, green) and $80^{\circ} \mathrm{C}$ (red, orange). The right side shows TOF distributions of $\mathrm{NO}(v=3, J=31.5)$ (blue, red) and $\mathrm{NO}(v=1, J=31.5)$ (green, orange) scattered from RFMS1 with a surface temperature of $25^{\circ} \mathrm{C}$ (blue, green) and $80^{\circ} \mathrm{C}$ (red, orange).

Table 6.1: Mean translational energy of $\mathrm{NO}(v=3)$ scattered from RFMS1.

\begin{tabular}{ccc}
\hline Rovibrational State & $\begin{array}{r}\langle E\rangle_{\text {trans }} \text { at } T_{\text {surf }}=25^{\circ} \mathrm{C} \\
\text { in meV }\end{array}$ & $\begin{array}{c}\langle E\rangle_{\text {trans }} \text { at } T_{\text {surf }}=80{ }^{\circ} \mathrm{C} \\
\text { in meV }\end{array}$ \\
\hline Incident $\mathrm{NO}(v=3, J=0.5)$ & $986 \pm 7$ & $986 \pm 7$ \\
$(v=3, J=6.5)$ & $230 \pm 34$ & $216 \pm 32$ \\
$(v=3, J=16.5)$ & $226 \pm 34$ & $220 \pm 33$ \\
$(v=3, J=31.5)$ & $184 \pm 28$ & $179 \pm 27$ \\
$(v=2, J=6.5)$ & $276 \pm 42$ & $283 \pm 43$ \\
$(v=2, J=16.5)$ & $268 \pm 40$ & $271 \pm 41$ \\
$(v=2, J=25.5)$ & $244 \pm 36$ & $254 \pm 38$ \\
$(v=1, J=31.5)$ & $253 \pm 37$ & $250 \pm 38$ \\
\hline
\end{tabular}



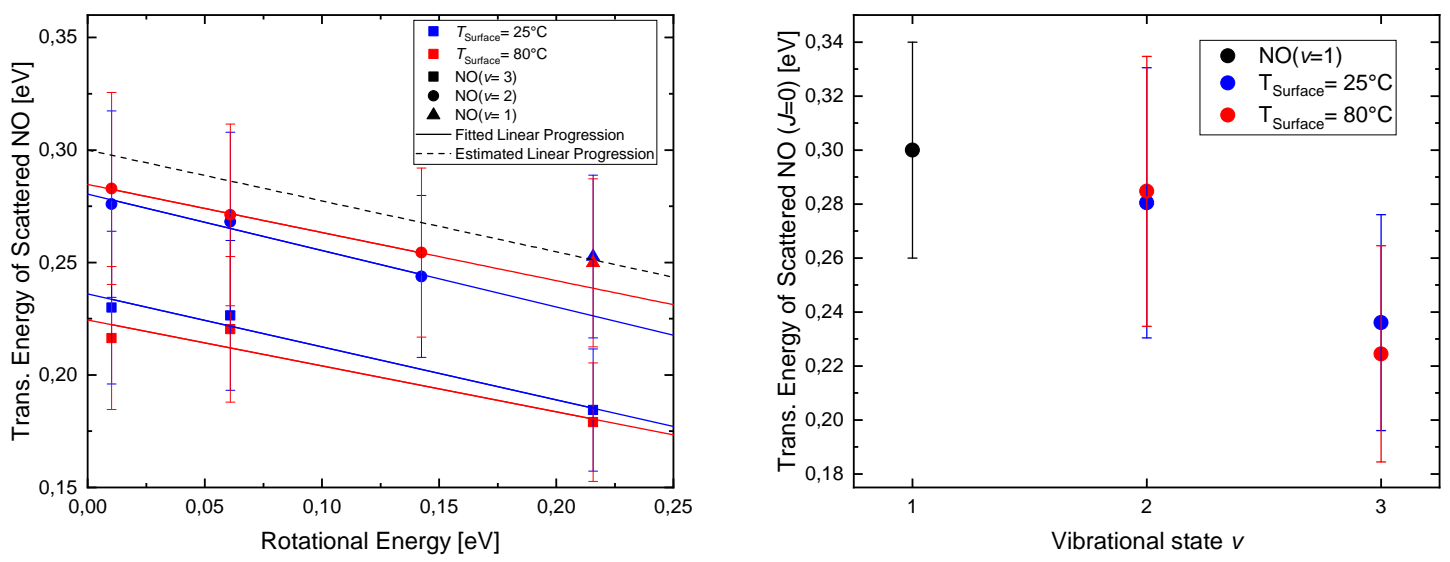

Figure 6.5: On the left side is the translational energy of scattered NO against the rotational energy is shown. Linear fits of the points of the $\gamma(1,3)$ and $\gamma(0,2)$ transition band are shown as compact lines. The estimated linear progression from the one evaluable point of the $\gamma(0,1)$ transition band is shown as a dashed line. On the right side the y-axis intercept of the linear progressions shown on the left are plotted against the vibrational state $v$. Points and lines are color-coded in respect to the surface temperature.

temperature of $\mathrm{VO}_{2}$. Table 6.1 shows the results from the TOF analysis. Figure 6.4 shows exemplary TOF distributions.

The TOF distributions of all vibrational states and surface temperatures show an intense component at early flight times. This early component stands clearly out of the rest of the TOF distribution with a width of about $10 \mu \mathrm{s}$. For $v=3$ and $v=1$ only a small tail component is seen at later flight times next to the early component. The tail component of the $\mathrm{NO}(v=3)$ TOF distributions is most pronounced for the low $J$ states. The tail component of the $\mathrm{NO}(v=2)$ TOF distributions is also more intense for lower $J$ states but also overall more intense than in the $\mathrm{NO}(v=3)$ distributions. In the case of all probed $v$ states the tail component is not present in TOF distribution of $\mathrm{NO}(J \geq 16.5)$.

Already in the TOF distributions it can be seen that the peaks of the higher $J$ state distributions are found at a later flight times than the lower $J$ state distributions of $v=3$. Furthermore, the TOF distributions show that the molecules in lower vibrational states arrive a few $\mu$ s earlier at the ionization region than it is shown in the corresponding distributions of $\operatorname{NO}(v=3)$. This is shown in the plot of the translational energies of scattered NO against their rotational energy on the left side of Figure 6.5. The points for $v=3$ and $v=2$ are fitted with a linear function and show a similar negative slope. Differences between the two surface temperatures are within the error bars. For $v=1$ only the high $J=31.5$ state distribution is observable because all other rotational lines 
are overshadowed by background signal. Therefore, the average slope of the other four data sets is drawn through the $\operatorname{NO}(v=1, J=31.5)$ points as a dashed line. On the right side of Figure 6.5 it can be seen that the translational energy of scattered NO increases with the loss of vibrational excitation.

\section{Conclusions}

The TOF distributions of $\mathrm{NO}(v=3)$ scattered from RFMS1 show a component with width and translational energy characteristic for the direct scattering channel. It is in all cases possible to resolve this direct scattering component, although another slower component is also present in most TOF distributions. This slow non-direct scattering component is more intense for lower $J$ states. This indicates that here rotational excitation is lower compared to the direct scattering component. The slow component is also much more intense in the TOF distributions of scattered $\mathrm{NO}(v=2)$. It can be assumed that some of the $\mathrm{NO}(v=3)$ which scatters non-directly also relaxes vibrationally and seen as the large tail distribution of $\mathrm{NO}(v=2)$. The tail distribution cannot be seen in the $v=1$ TOFs because only the high $J=31.5$ state is measurable.

The plots of translational energy of scattered NO against rotational energy of $v=3$ and $v=2$ follow linear progressions with negative slope. Also it can be seen that the translational energy of scattered $\mathrm{NO}(v=3)$ is offset by about $-0.05 \mathrm{eV}$ in respect to the translation energies of $\mathrm{NO}(v=2)$. Therefore, the translational energy of the incident $\mathrm{NO}(v=3)$ is converted to rotational excitation while the initial vibrational excitation is transferred to the translational energy during scattering. This behavior is reminiscent of the direct scattering channel. Therefore, equilibration between the surface and the molecules does not occur because the interaction time is too low.

The thesis of Kai Golibrzuch [42] shows a similar graph (Figure 3.25 in his thesis) for $\mathrm{NO}(v=3)$ scattering from $\mathrm{Au}(111)$. For scattering from $\mathrm{Au}(111)$ more than half of the translational energy is retained. In the case of $\mathrm{NO}(v=3)$ scattering from $\mathrm{VO}_{2}$ less than a third of the translational energy of the incident $\mathrm{NO}$ is retained after scattering. This shows the higher effective mass of the gold surface during NO scattering. It is shown that RFMS1 shows the same direct scattering properties as a $\mathrm{Au}(111)$ surface. Both surface just show different loss of translational energy in NO because of the different effective masses. 


\subsubsection{REMPI Spectra of Scattered NO}

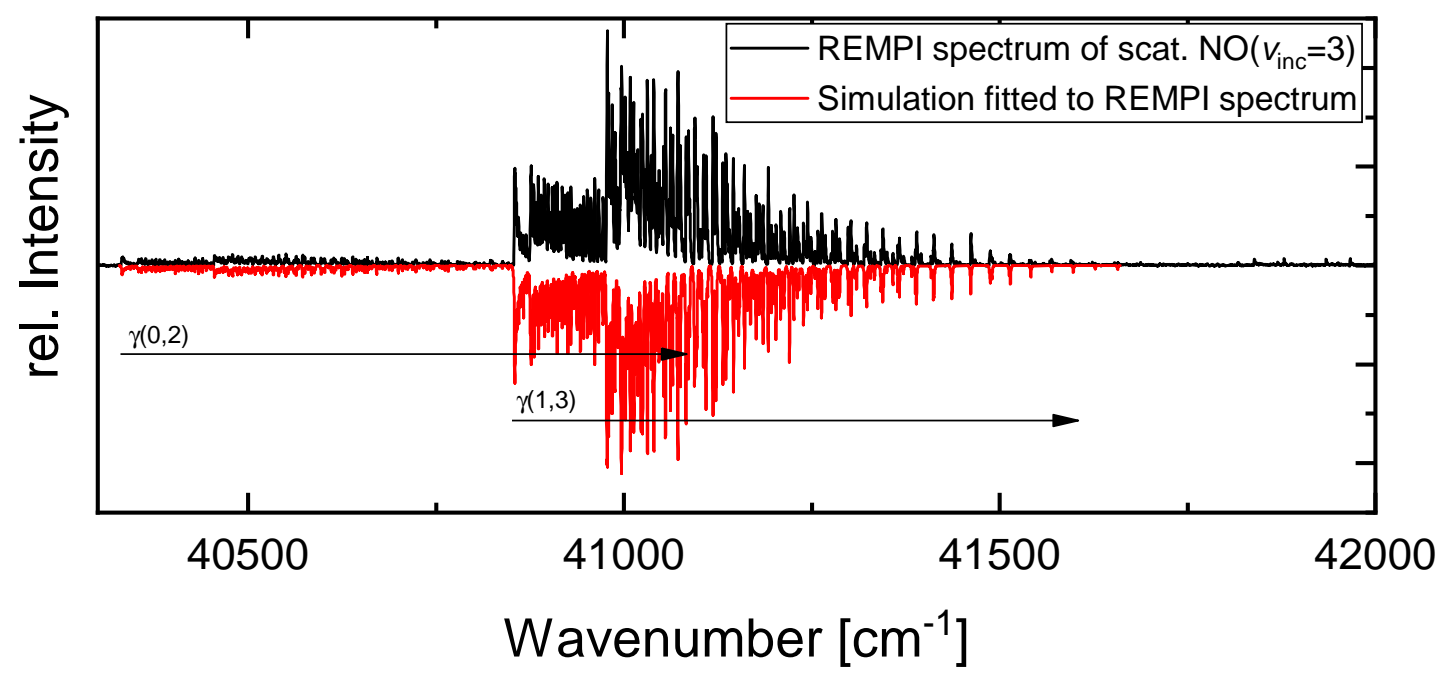

Figure 6.6: REMPI spectrum of $\mathrm{NO}\left(v_{\text {inc }}=3\right)$ scattered from RFMS1 at a surface temperature of $45^{\circ} \mathrm{C}$. In this spectral region the transition bands $\gamma(1,3)$ and $\gamma(0,2)$ can be observed. The black line shows the experimental data. The red line shows the simulation of the observed transition bands fitted to the experimental data.

To probe each relevant rotational line at once REMPI spectra of the $\gamma(1,3), \gamma(0,2)$ and $\gamma(0,1)$ transition bands were measured. An example of such a REMPI spectrum is shown in Figure 6.6. They are analyzed for the vibrational and rotational state populations and subsequently also rotational temperatures. The results of the vibrational population analysis are shown for both surfaces in Figure 6.7. It can be seen in the bar plots of the upper half of that figure that for both surfaces and both surface temperatures more than $97.5 \%$ of the NO molecules remain in their initial vibrational state after scattering. The leftover population consists almost exclusively of $\operatorname{NO}(v=2)$. After background correction no intensity of the $\gamma(0,1)$ band could be detected. Therefore, the population of $v=1$ in the scattered NO was assumed as zero. Transition bands of $v=0$ could not be measured because of the high background of $\operatorname{NO}(v=0)$ in the molecular beam. In the measured spectral range also the $\gamma(2,4)$ transition band can be found if $\operatorname{NO}(v=4)$ is present. It is not observed in any spectrum so it is safe to assume that also no population is found in $v=4$. Therefore, no vibrational excitation of $\mathrm{NO}(v=3)$ to $\mathrm{NO}(v=4)$ occurs during the scattering from $\mathrm{VO}_{2}$.

For both surfaces the population of the initial vibrational state $v=3$ is slightly reduced above the MIT temperature of $68^{\circ} \mathrm{C}$. This effect becomes more apparent in the plots in the lower half of Figure 6.7. For RFMS1 the relative population of $\mathrm{NO}(v=3)$ decreases 

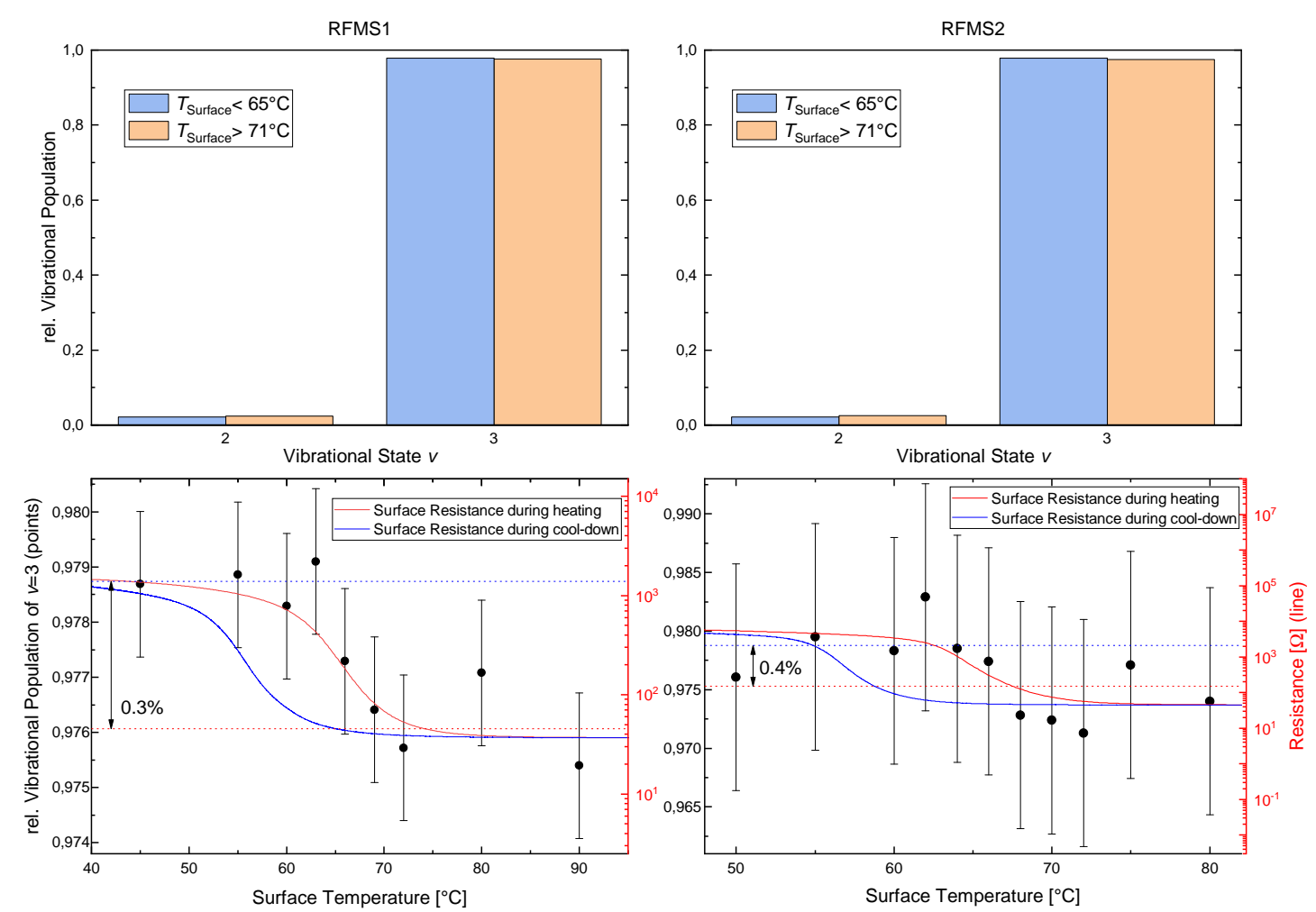

Figure 6.7: Relative vibrational distributions of $v=2$ and $v=3$ for NO scattered (top) from RFMS1 (left) and RFMS2 (right) at a surface temperature $<65^{\circ} \mathrm{C}$ and $>71{ }^{\circ} \mathrm{C}$ with the relative vibrational population of $\mathrm{NO}(v=3)$ plotted against the surface temperature of RFMS1 and RFMS2 (bottom). In the background of the data is the corresponding resistance against surface temperature measurement as visual aid shown. The red compact line is for positive heating rate and the blue compact line for negative heating rate. The dotted lines are the average value of the data points $<65^{\circ} \mathrm{C}$ (blue) and $>71^{\circ} \mathrm{C}$ (red).

by $0.3 \%$ with the MIT. By comparison with the resistance against surface temperature plot in the same graph it can be seen that this progression occurs parallel to the MIT when the surface temperature is linearly increased (red solid line). The measurement of NO scattered from RFMS2 was expected to yield more clear results. It has a larger MIT contrast than RFMS1 and also had no argon entrapped in its lattice. For unknown reasons, the noise in the data points is larger than in the data set of RFMS1 so that the error bars exceed the observed transition. Nevertheless, the difference of the average of the points above and below the MIT temperature show a similar trend as can be seen with RFMS1. The population of the initial vibrational state $v=3$ decreases by $0.4 \%$. The transition between the two different relaxation probabilities occurs also along MIT when the surface temperature is linearly increased (red solid line). 

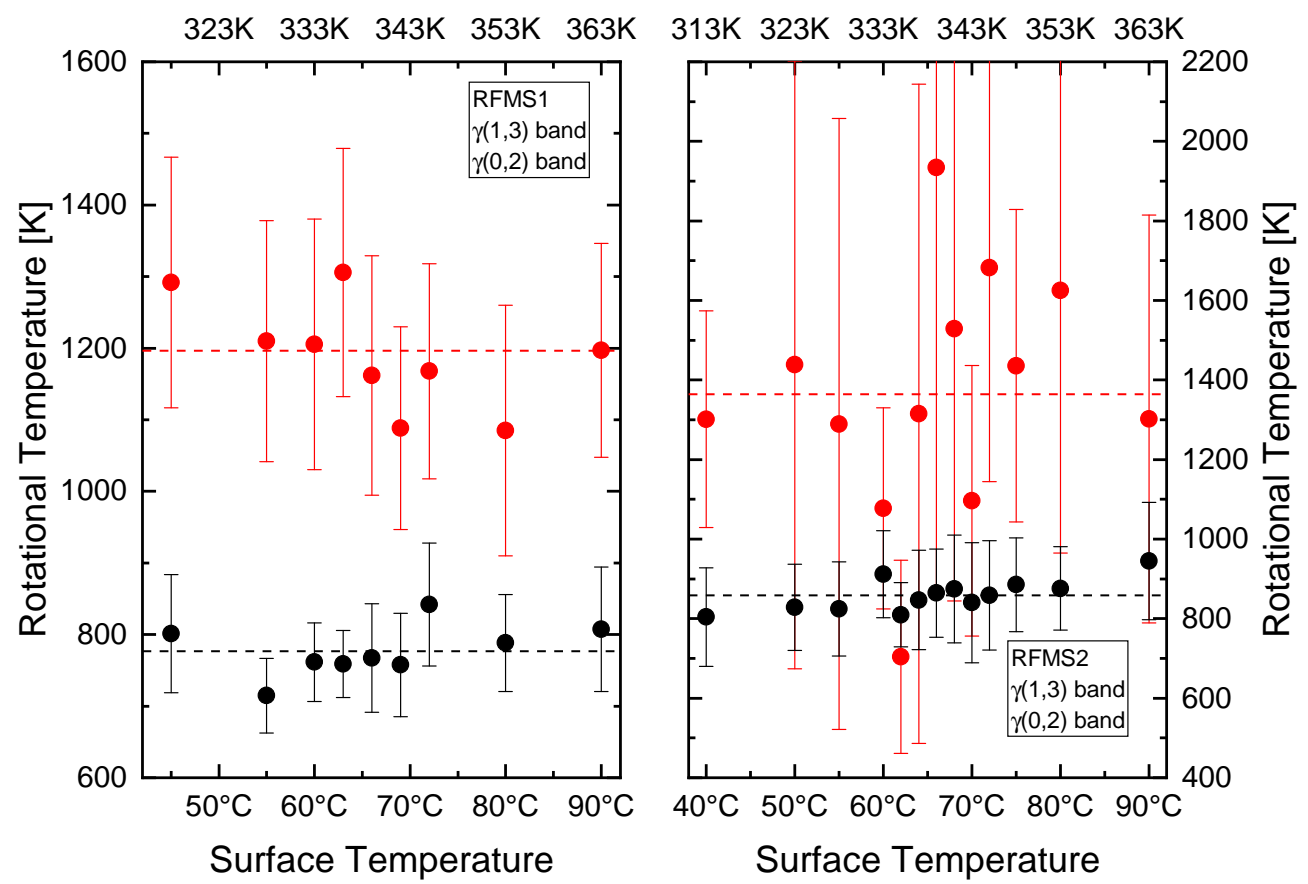

Figure 6.8: Rotational temperatures of the $\gamma(1,3)$ and $\gamma(0,2)$ band of NO scattered from RFMS1 and RFMS2 plotted over the surface temperature. Dashed lines mark the respective average rotational temperatures.

The $\gamma(1,3)$ and $\gamma(0,2)$ band that can be seen in the REMPI spectra are analyzed for their rotational temperature. The averaged results are listed in Table 6.2 and the individual values are plotted in Figure 6.8. For both surfaces the rotational temperatures of the $\gamma(0,2)$ band is with an average of $1200 \mathrm{~K}$ for RMFS1 and $1400 \mathrm{~K}$ for RMFS2 higher than the rotational temperatures of the $\gamma(1,3)$ band. For $\gamma(1,3)$ average rotational temperatures of $780 \mathrm{~K}$ for RMFS1 and $860 \mathrm{~K}$ for RMFS2 are found. All measured rotational temperatures are in any case significantly hotter than the surface temperature which ranges from $313 \mathrm{~K}$ to $363 \mathrm{~K}$. The plots show a small rise in rotational temperature along the surface temperature of the $\gamma(1,3)$ band for both surfaces which is in the case of RFMS1 accompanied by a small drop in rotational temperature of the $\gamma(0,2)$ band. The difference is however smaller than the error bars and in consequence considered as not significant. 
Table 6.2: Rotational mean energies $\langle E\rangle_{\text {rot }}$ and temperatures $T_{\text {rot }}$ of the $\gamma(0,2)$ and $\gamma(1,3)$ transition band of NO scattered from RFMS1 and RFMS2.

\begin{tabular}{ccccc}
\hline Surface & $\langle E\rangle_{\text {rot }}(v=2)[\mathrm{meV}]$ & $T_{\text {rot }}(v=2)[\mathrm{K}]$ & $\langle E\rangle_{\text {rot }}(v=3)[\mathrm{meV}]$ & $T_{\text {rot }}(v=3)[\mathrm{K}]$ \\
\hline RFMS1 & $67 \pm 2$ & $1200 \pm 80$ & $49 \pm 2$ & $780 \pm 40$ \\
RFMS2 & $73 \pm 5$ & $1400 \pm 310$ & $51 \pm 3$ & $860 \pm 50$ \\
\hline
\end{tabular}

\section{Conclusions}

Since the rotational temperatures are significantly higher than the surface temperature and also do not noticeably increase with the surface temperature it can be assumed that the scattered NO molecules are not thermally equilibrated with the surface and scatter directly from it. Remarkable is that NO in the initial vibrational state $v=3$ is found with much lower rotational excitation than the relaxed $\mathrm{NO}(v=2)$. This effect appears to be contrary to what was observed in previous scattering experiments of $\mathrm{NO}(v=3)$ from $\mathrm{Au}(111)^{73}$. There, the relaxed $v$ states of scattered $\mathrm{NO}$ and $\mathrm{CO}$ show lower rotational excitation than the molecules that retain their initial vibrational state.

The observed transition in relaxation probability of $\mathrm{NO}(v=3)$ shown in Figure 6.7 is comparable to the difference in relaxation probability of $\mathrm{NO}(v=2)$ seen after $11 \mu \mathrm{s}$ flight time in Figure 4.13. In contrast to the experiments with $\mathrm{NO}(v=2)$ it is shown that this change in relaxation probability occurs along the change in electrical resistance accompanying the MIT of $\mathrm{VO}_{2}$. This is the first clear sign that the relaxation probability of scattered $\mathrm{NO}(v=3)$ is affected by the MIT of $\mathrm{VO}_{2}$. The relaxation probability changes by $0.3 \%$ for RFMS1 and $0.4 \%$ for RFMS2 along the MIT.

On the other hand the observed relaxation probability and its dependence on the MIT is lower than expected. As shown in Figure 2.13 the vibrational relaxation probability of $\mathrm{NO}(v=3)$ scattered from the highly electrically conductive $\mathrm{VO}_{2}(\mathrm{R})$ should occur with more than $75 \%$ as shown in Figure 6.9. This expectation is done under the assumption that it behaves like other metals such as gold and silver. The relaxation probability is also expected to decrease during the transition to $\mathrm{VO}_{2}(\mathrm{M})$ by either $75 \%$ to zero or by about $10 \%$ depending on the assumptions made. Such a change in vibrational relaxation probability could not be observed with $\mathrm{NO}(v=3)$ scattered from RFMS1 or RFMS2. 


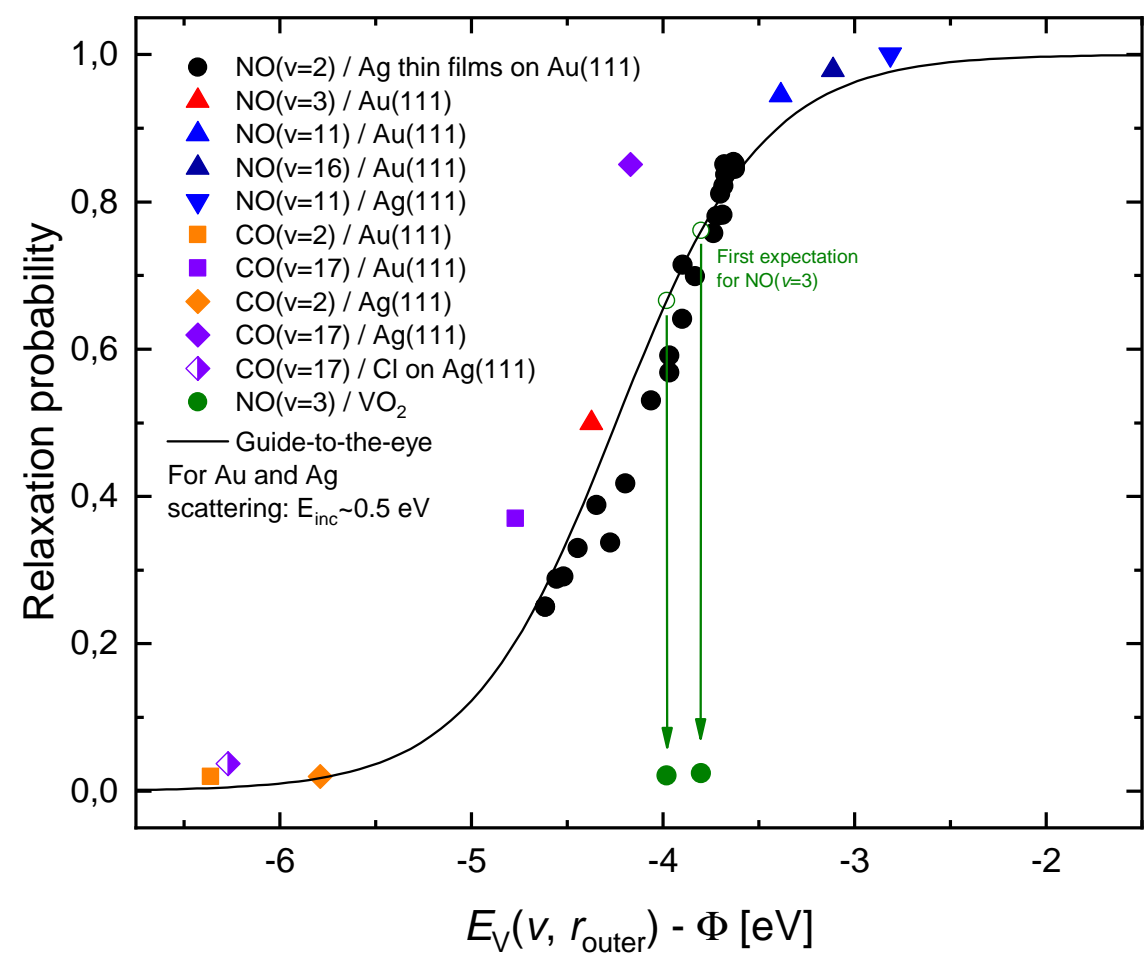

Figure 6.9: Graph of relaxation probability for vibrationally excited NO and CO from different metal surfaces as shown in Figure 2.13 with added points for $\mathrm{NO}(v=3)$ scattered from $\mathrm{VO}_{2}$. The empty dots are expectations for $v=3$ scattered from $\mathrm{VO}_{2}$ based on the general trend seen from the other points.

\subsection{Scattering of $\mathrm{NO}(v=11)$ from RFMS3}

\subsubsection{Sample Characterization of RFMS3}

Further NO scattering experiments are performed with $\mathrm{NO}(v=11)$ scattering from a RFMS prepared $\mathrm{VO}_{2}$ thin film. The sample is labeled as RFMS3 and consists of a rectangular slab of $\mathrm{VO}_{2}$ deposited on a r-cut sapphire substrate. To clean the surface it was sputtered before the day of the experiments three times with $\mathrm{O}_{2}^{+}$-ions accelerated to $1 \mathrm{kV}$ for $5 \mathrm{~min}$ each. At the beginning of each measurement day the sample was again sputtered with $\mathrm{O}_{2}^{+}$-ions accelerated to $1 \mathrm{kV}$ for $5 \mathrm{~min}$. The characteristics of this sample are shown in Figure 6.10, RFMS3 looks similar to RFMS1 and RFMS2. It has a flat and reflective colorless surface with no visible defects as shown in Figure 6.10 (b). The resistance against surface temperature plot shown in Figure 6.10 (a) shows that the MIT contrast is the most intense of all samples used for this work. The dashed lines show the resistance of RFMS3 before sputtering and the solid line shows it after sputtering. The plot before sputtering is slightly offset in both $\mathrm{x}$ and $\mathrm{y}$ direction which can be attributed 

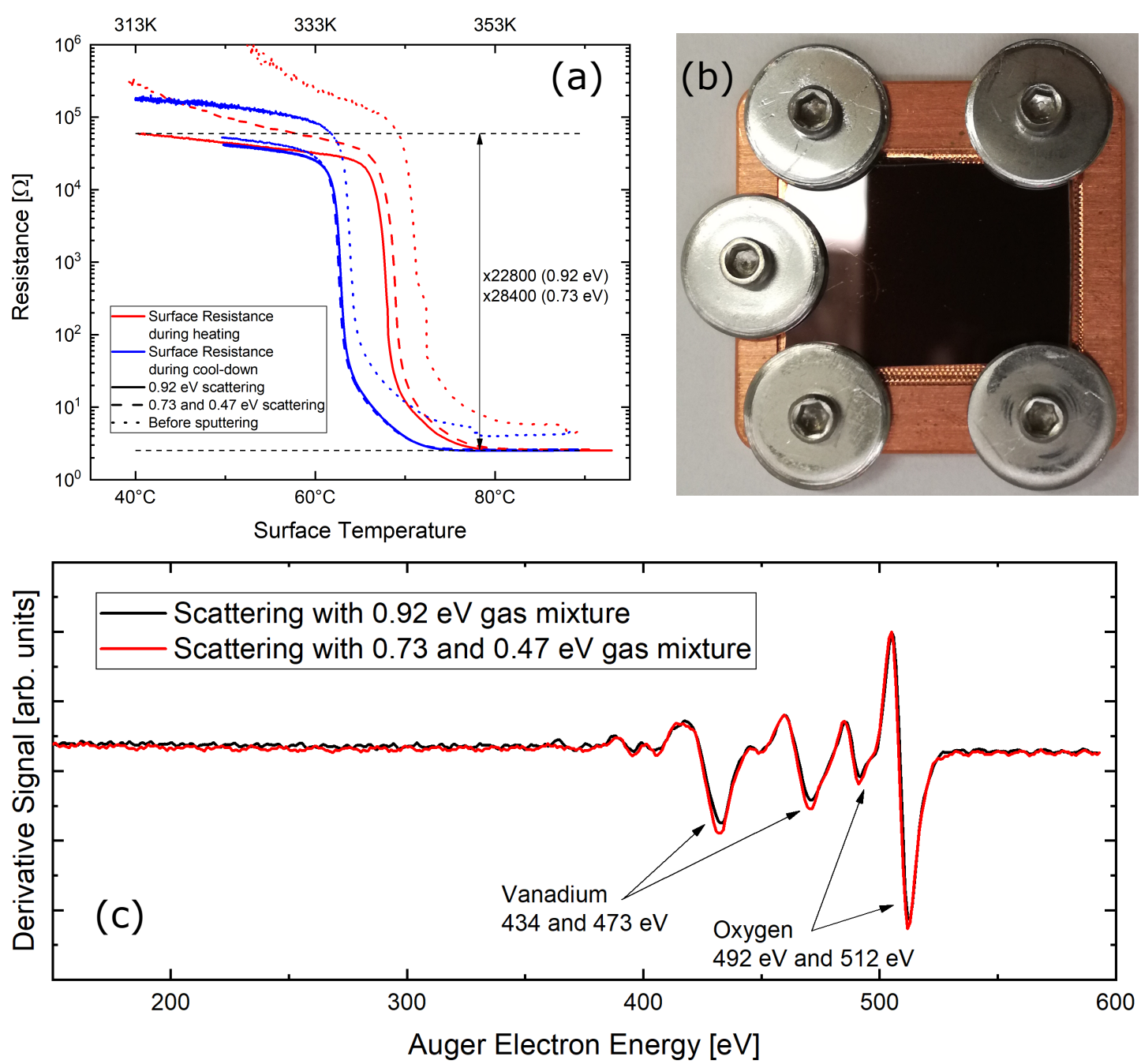

Figure 6.10: Sample characteristics of RFMS3. (a) shows the surface resistance against temperature plot of RFMS3 before and after sputtering with $1 \mathrm{kV} \mathrm{O}_{2}{ }^{+}$-ions. (b) shows an image of the $\mathrm{VO}_{2}$ thin film. (c) shows the AES of RFMS3 before experimental data was measured.

to differences in the experimental and electronic setup since the measurement were done almost 4 months apart. Yet it can be seen that the MIT contrast between all three plots remains in consideration of a reasonable scatter basically unchanged despite the time the surface was exposed to UHV conditions and sputtering. Furthermore, in Figure 6.10 (c) it can be seen that the $\mathrm{O}_{2}^{+}$-ion sputtering removed all traces of carbon contamination from the surface so that only AES signals corresponding to vanadium and oxygen can be detected. 


\subsubsection{Angular Distributions of Scattered NO}

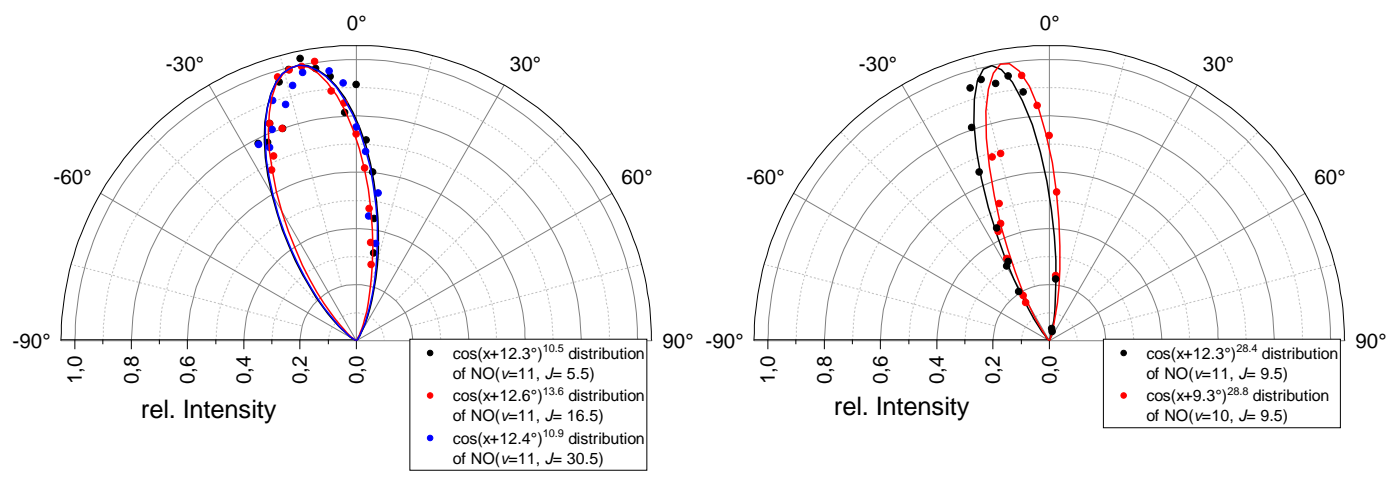

Figure 6.11: Angular distributions of the $\mathrm{R}_{22}(5.5), \mathrm{Q}_{11}(16.5)$ and $\mathrm{Q}_{22}(30.5)$ lines of the $\gamma(4,11)$ band (left) and the $\mathrm{Q}_{11}(9.5)$ lines of the $\gamma(4,11)$ and $\gamma(3,10)$ bands (right) after scattering from RFMS3.

The left side of Figure 6.11 shows the angular distribution of scattered NO probed at three different transition lines of the $\gamma(4,11)$ band. It can be seen that all probed transition lines show similar angular offsets and angular spreads. This is a strong indication that NO in the low $J$ states as in the high $J$ states scatters along the same channel. The narrow cos distribution with an exponent of $>10$ suggests that this channel is the direct scattering channel. On the right side of the same figure a comparison is shown between the angular distributions of NO probed via the $\gamma(4,11)$ and $\gamma(3,10)$ band. The former depicts NO molecules that retain their initial vibrational state $v=11$ and the latter depicts vibrationally relaxed NO. For both transition bands the $\mathrm{Q}_{11}(9.5)$ transition line of the respective band is probed. It shows that the angular distribution is in general similar but the angular offset is shifted by about $3^{\circ}$. Since the two angular distributions were measured one week apart it is possible that the mounting respectively heating wires have deformed in the mean time. This may lead to a slight change in the tilting of the surface. The angular distributions shown on the left side of Figure 6.11 were all three measured on the same day and show no angular offset in respect to each other. There is no physical reason why $\mathrm{NO}(v=10)$ should scatter of at a different angle than $\mathrm{NO}(v=11)$. 


\subsubsection{Time-of-Flight Spectroscopy of Scattered NO}
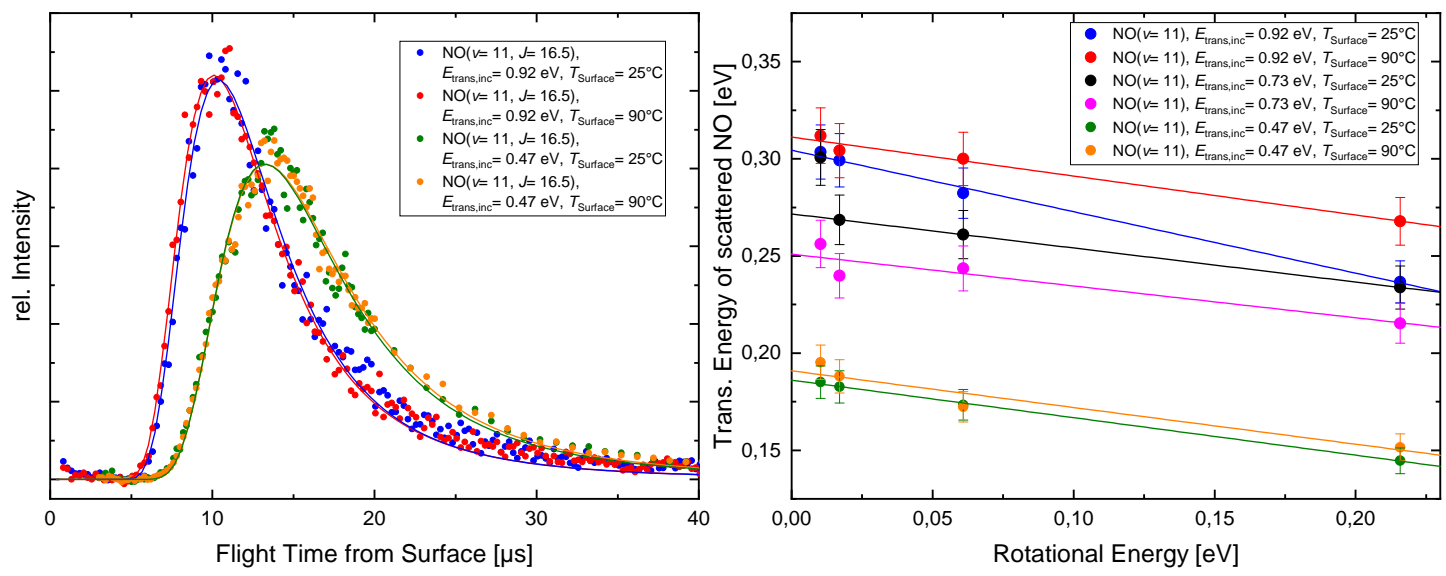

Figure 6.12: Left Side: Exemplary TOF distributions of $\mathrm{NO}(16.5)$ scattered from RFMS3 with translational energies of 0.92 and $0.47 \mathrm{eV}$ in the incident molecular beam.

Right Side: Translational energy of scattered NO molecules plotted against their rotational energy for incident molecular beam pulses with different energies. Surface temperatures of $25^{\circ} \mathrm{C}$ and $90^{\circ} \mathrm{C}$ are probed. Each data set is fitted with a linear progression.

Exemplary TOF distributions are shown on the left side of Figure 6.12. The distributions are fitted with Equation 3.5. No tail distribution appears in any of the $\mathrm{NO}(v=11)$ TOF distributions of NO scattered from RFMS3. The width of the distributions is about 10 us.

On the right side of the same figure the mean translational energies of $\mathrm{NO}(v=11)$ molecules scattered from RFMS3 are plotted against their rotational energy. The translational energies show no difference when the molecules are scattered from $\mathrm{VO}_{2}(\mathrm{M})$ or $\mathrm{VO}_{2}(\mathrm{R})$. The translational energy of scattered NO increases for higher translational energies of the incident molecular beam and decreases for higher rotational energies. The plotted translational energies of incident and scattered NO are listed in Table 6.3.

Figure 6.13 shows a Baule plot of for rotational excitation adjusted translational energy of scattered NO plotted against the translational energy of incident NO. Scattering from $\mathrm{VO}_{2}(\mathrm{M})$ at a surface temperature of $25^{\circ} \mathrm{C}$ is shown in blue and $\mathrm{NO}$ scattering from $\mathrm{VO}_{2}(\mathrm{R})$ at a surface temperature of $90^{\circ} \mathrm{C}$ is shown in red. Linear progressions are fitted to both data sets which lie close to each other. This shows that for translational energy loss during scattering it makes no difference if the surface is in its monoclinic or its rutile phase. The effective surface mass gained from the slopes of the linear progressions are $m(\operatorname{RMFS} 3(\mathrm{M}))=95 \mathrm{amu}$ and $m(\operatorname{RMFS} 3(\mathrm{R}))=91 \mathrm{amu}$. Considering the error of the 


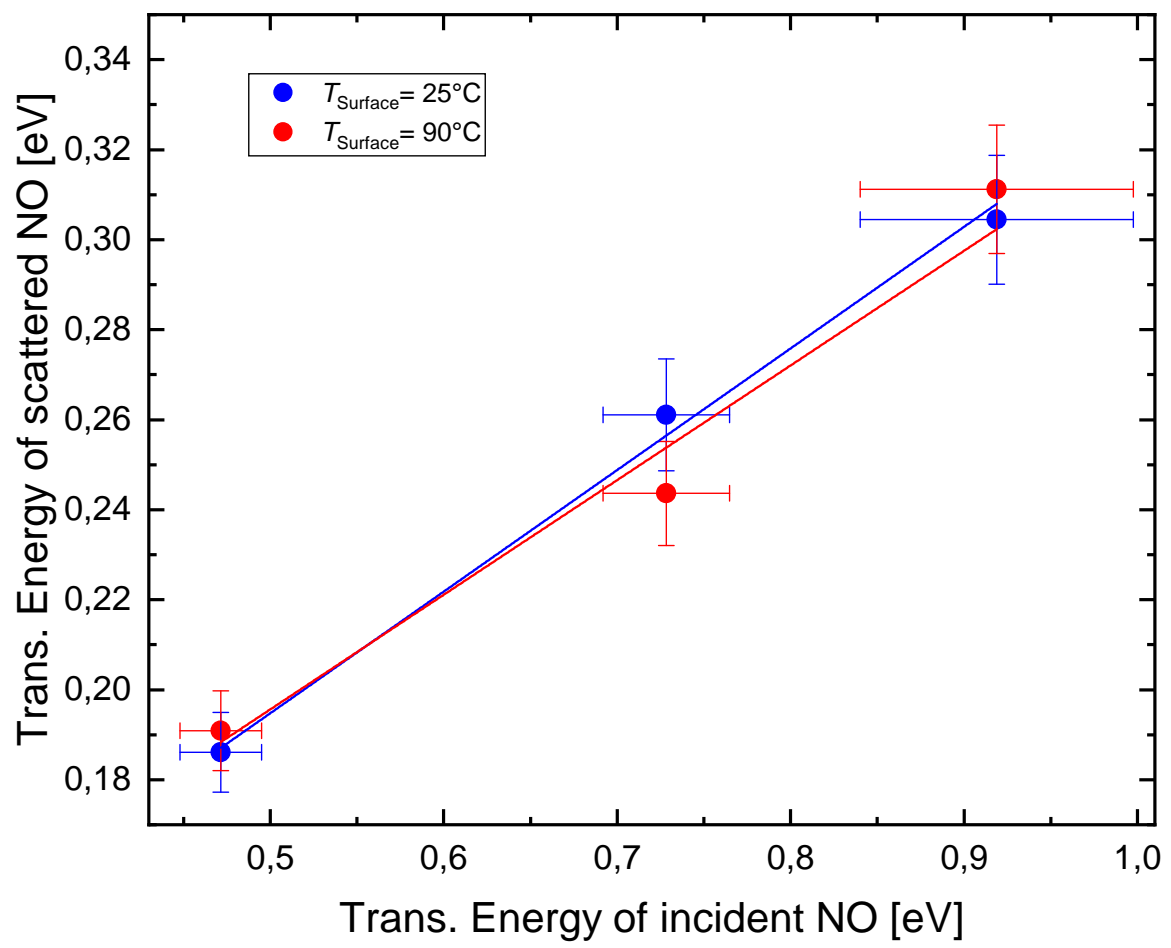

Figure 6.13: Baule plot of for rotational excitation adjusted translational energy of NO scattered from RFMS3 against the corresponding translational energy of incident NO. The slope of the linear progression fitted to the data points corresponds to an effective surface mass of $m(\operatorname{RMFS} 3(\mathrm{M}))=95 \mathrm{amu}$ (blue) and $m(\operatorname{RMFS} 3(\mathrm{R}))=91 \mathrm{amu}($ red).

Table 6.3: Mean translational energies of $\mathrm{NO}(v=11)$ scattered from RFMS3.

\begin{tabular}{ccccc}
\hline Rovibrational State & $\begin{array}{c}T_{\text {Surf }} \\
\text { in }{ }^{\circ} \mathrm{C}\end{array}$ & $\begin{array}{c}\langle E\rangle_{\text {trans,scat }} \\
\text { in meV }\end{array}$ & $\begin{array}{c}\langle E\rangle_{\text {trans,scat }} \\
\text { in meV }\end{array}$ & $\begin{array}{c}\langle E\rangle_{\text {trans,scat }} \\
\text { in meV }\end{array}$ \\
\hline Incident $\mathrm{NO}(v=11, J=0.5)$ & - & $920 \pm 79$ & $728 \pm 37$ & $471 \pm 24$ \\
$(v=11, J=5.5)$ & 25 & $304 \pm 14$ & $301 \pm 15$ & $185 \pm 9$ \\
$(v=11, J=9.5)$ & 25 & $299 \pm 14$ & $269 \pm 13$ & $183 \pm 9$ \\
$(v=11, J=16.5)$ & 25 & $282 \pm 13$ & $261 \pm 13$ & $173 \pm 8$ \\
$(v=11, J=30.5)$ & 25 & $237 \pm 11$ & $234 \pm 12$ & $145 \pm 7$ \\
$(v=11, J=5.5)$ & 90 & $312 \pm 15$ & $256 \pm 13$ & $195 \pm 9$ \\
$(v=11, J=9.5)$ & 90 & $304 \pm 14$ & $240 \pm 12$ & $188 \pm 9$ \\
$(v=11, J=16.5)$ & 90 & $300 \pm 14$ & $244 \pm 12$ & $172 \pm 8$ \\
$(v=11, J=30.5)$ & 90 & $268 \pm 13$ & $215 \pm 11$ & $151 \pm 7$ \\
\hline
\end{tabular}


translational energies these values are very close to the effective mass of CVD2 from Figure 4.6 .

\section{Conclusions}

RFMS3 can be considered as the $\mathrm{VO}_{2}$ thin film best suited for $\mathrm{NO}$ scattering experiments shown in this work. This is caused by a combination of the high order in the RFMS prepared thin film and its high flatness. Also the exclusive sputtering with $\mathrm{O}_{2}{ }^{+}$ ions preserves the surface's chemical integrity. NO scatters exclusively along the direct channel from RFMS3 which averts the influence of other interfering scattering channels. It is also shown that for the loss of translational energy during scattering it makes no difference if the thin film is in the $\mathrm{VO}_{2}(\mathrm{M})$ or $\mathrm{VO}_{2}(\mathrm{R})$ phase.

\subsubsection{REMPI Spectra of Scattered NO}

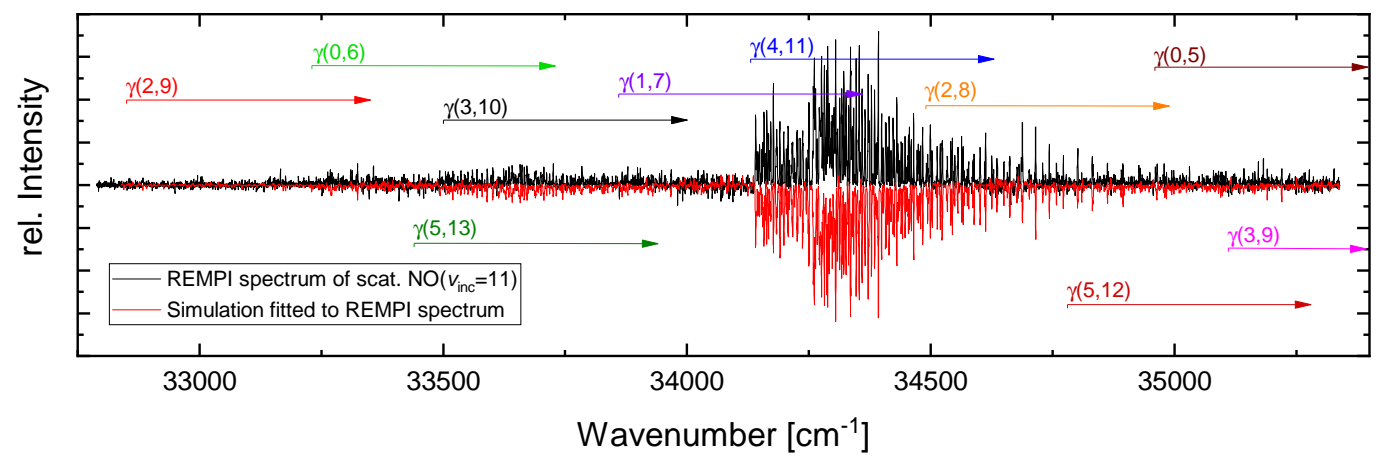

Figure 6.14: Background corrected, exemplary REMPI spectrum of $\mathrm{NO}(v=11)$ scattered from RFMS3 in black and simulation fitted to experimental data. Arrows indicate transition bands.

To gain distributions of as many vibrational states as possible the dye laser used to generate the REMPI beam was run with Pyrromethene 597 (Exciton Inc.). This laser dye, when doubled, covers the wavelengths of the vibrational transition bands $\gamma(0,5)$, $\gamma(0,6), \gamma(1,7), \gamma(2,8), \gamma(2,9), \gamma(3,9), \gamma(3,10), \gamma(4,11), \gamma(5,12)$ and $\gamma(5,13)$. An exemplary REMPI spectrum with the corresponding fitted simulation and marked positions of the vibrational bands is shown in Figure 6.14. From this figure it can already be seen that mostly the initial vibrational state of $\mathrm{NO}(v=11)$ is populated with a small amount found in $\mathrm{NO}(v=10)$. All other transition bands barely exceed the background noise of the detector.

Vibrational population distributions for all three probed translational energies of the incident molecular beam pulse $E_{\text {trans,inc }}$ of $\mathrm{NO}(v=11)$ are shown on the left side of 

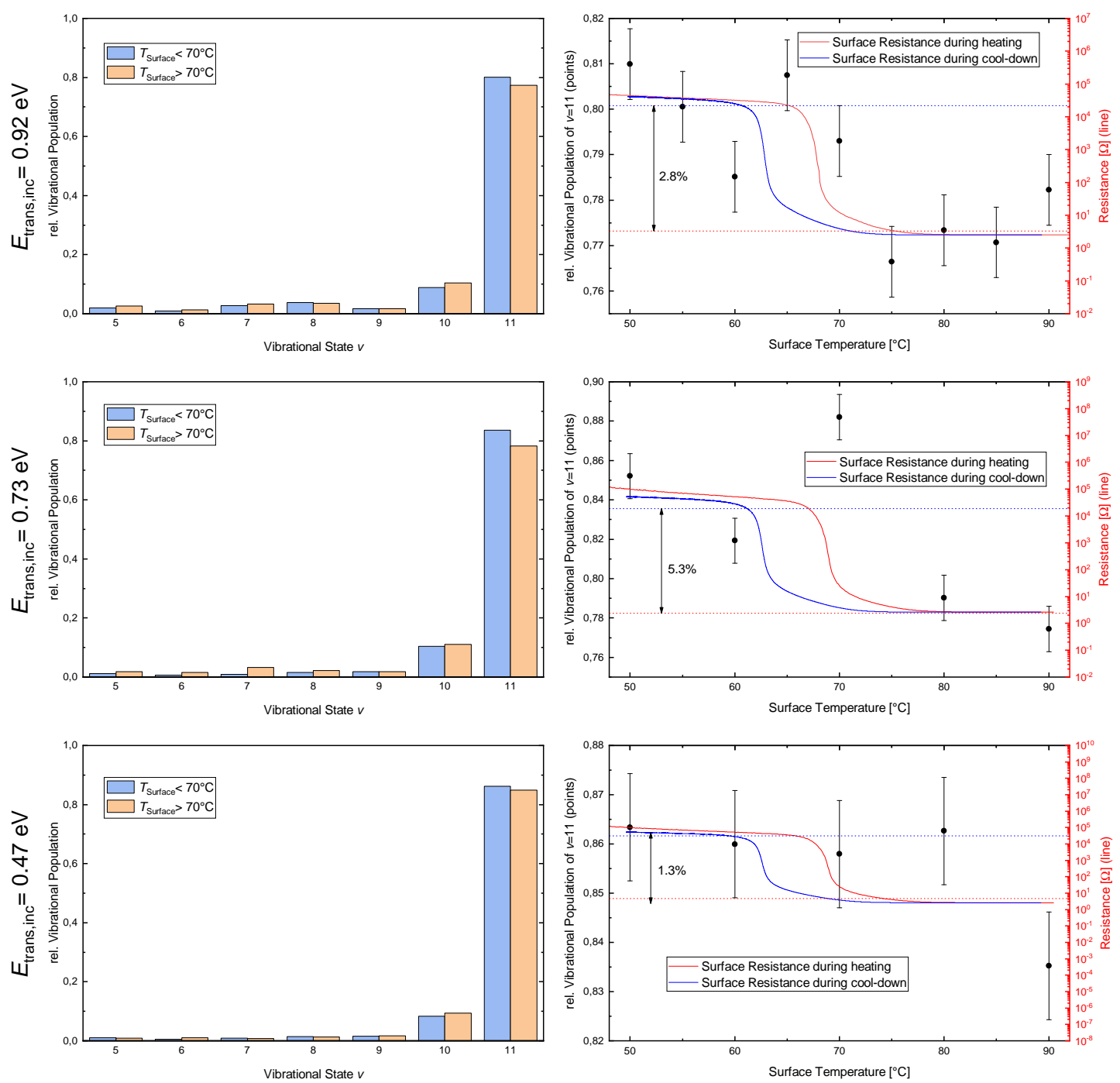

Figure 6.15: Vibrational distributions for all measured vibrational transition bands for surface temperatures above and below the MIT and relative vibrational population of $\operatorname{NO}(v=11)$ plotted over surface temperature for all measured incident translational energies.

Figure 6.15. About $77 \%$ to $86 \%$ of the total vibrational population is found in the $v=11$ state. The population of $v=11$ increases for smaller translational energies of the incident molecular beam. Furthermore, for all translational energies the population of $v=11$ is bigger for a surface temperature below the MIT temperature and lower above the MIT temperature. About $10 \%$ of the total number of detected molecules populate $\mathrm{NO}(v=10)$. The population of scattered $\mathrm{NO}(v=10)$ from $\mathrm{VO}_{2}(\mathrm{M})$ is in all cases smaller than the population of $\mathrm{NO}(v=10)$ from $\mathrm{VO}_{2}(\mathrm{R})$. The population of $\mathrm{NO}(v=10)$ shows no obvious dependence on the translational energy. The rest of the 

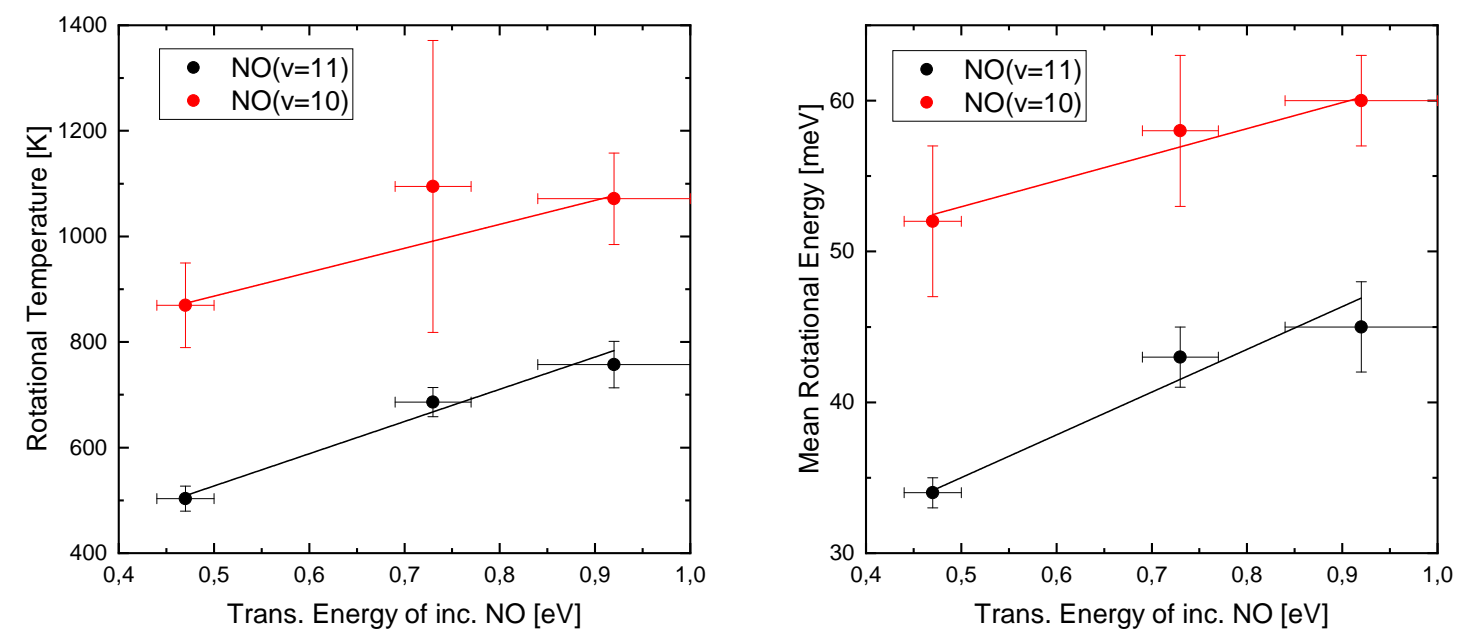

Figure 6.16: Rotational temperature (left side) and mean rotational energy (right side) of the $\gamma(4,11)$ and $\gamma(3,10)$ transition band of $\mathrm{NO}(v=11)$ scattered from $\mathrm{VO}_{2}$. Linear progressions are added to the data points to illustrate the linear dependence between rotational excitation and translational energy of incident NO.

total population is found in the vibrational states from 5 to 9 . These vibrational states $\mathrm{NO}(v<10)$ are more populated with higher translational energy. In most cases the population of these lower states increases if the surface temperature is above the MIT temperature.

On the right side of Figure 6.15 are the relative vibrational populations of $\operatorname{NO}(v=11)$, corresponding to the vibrational population distribution shown on the left, plotted against the surface temperature $T_{\text {surf }}$ of RFMS3. For the incident molecular beam with $E_{\text {trans,inc }}=0.92 \mathrm{eV} 10$ points were taken while for the other two only 5 points were measured. In all cases the vibrational population of scattered $\operatorname{NO}(v=11)$ is reduced when scattered from $\mathrm{VO}_{2}(\mathrm{R})$ compared to scattering from $\mathrm{VO}_{2}(\mathrm{M})$. The average

Table 6.4: Mean rotational excitation energy $\langle E\rangle_{\text {rot }}$ and rotational temperatures $T_{\text {rot }}$ from REMPI spectra of NO scattered from RFMS3 with different incident translational energies.

\begin{tabular}{ccccc}
\hline $\begin{array}{c}E_{\text {inc,trans }} \\
\text { in } \mathrm{meV}\end{array}$ & $\begin{array}{c}\langle E\rangle_{\text {rot }}(v=10) \\
\text { in } \mathrm{meV}\end{array}$ & $\begin{array}{c}T_{\text {rot }}(v=10) \\
\text { in } \mathrm{K}\end{array}$ & $\begin{array}{c}\langle E\rangle_{\text {rot }}(v=11) \\
\text { in } \mathrm{meV}\end{array}$ & $\begin{array}{c}T_{\text {rot }}(v=11) \\
\text { in } \mathrm{K}\end{array}$ \\
\hline $920 \pm 80$ & $60 \pm 3$ & $1100 \pm 90$ & $45 \pm 3$ & $760 \pm 50$ \\
$730 \pm 40$ & $58 \pm 5$ & $1100 \pm 280$ & $43 \pm 2$ & $690 \pm 30$ \\
$470 \pm 30$ & $52 \pm 5$ & $870 \pm 90$ & $34 \pm 1$ & $510 \pm 30$ \\
\hline
\end{tabular}


of the relative vibrational population of $\mathrm{NO}(v=11)$ is indicated by a blue dotted line for values with $T_{\text {surf }}<70^{\circ} \mathrm{C}$ and by a red dotted line for values with $T_{\text {surf }}>70^{\circ} \mathrm{C}$. The measured differences between these two states is $2.8 \%$ for $E_{\text {trans,inc }}=0.92 \mathrm{eV}$. For $E_{\text {trans,inc }}=0.73 \mathrm{eV}$ a difference of $5.3 \%$ is observed, though the population of $v=11$ at $T_{\text {surf }}>70^{\circ} \mathrm{C}$ might be underestimated. The cause for this is the high population of $v=7$ at $T_{\text {surf }}>70^{\circ} \mathrm{C}$. It appears to be an outlier. A difference of $1.3 \%$ is observed for $E_{\text {trans,inc }}=0.47 \mathrm{eV}$. It has to be noted that the value for $T_{\text {surf }}=80^{\circ} \mathrm{C}$ appears to be an outlier and if it is ignored the transition in relative vibrational population of $\mathrm{NO}(v=11)$ might be twice as large. This way the change in vibrational relaxation probability of $\mathrm{NO}(v=11)$ scattered with $E_{\text {trans,inc }}=0.47 \mathrm{eV}$ from RFMS3 would fall more in line with the other two translational energies.

From the rotational state distribution taken from the same REMPI spectra also the rotational excitation are analyzed for the $\gamma(3,10)$ and $\gamma(4,11)$ transition band. The intensity of all other transition bands is too low to be analyzable. The rotational temperatures and mean rotational energies are shown in Table 6.4 and Figure 6.16. The rotational temperatures both of $\mathrm{NO}(v=11)$ and $\mathrm{NO}(v=10)$ increase with the translational energy of the incident molecular beam. The rotational temperature are in general more than $50 \%$ larger for $\mathrm{NO}(v=10)$ than for $\mathrm{NO}(v=11)$. The mean rotational energies of both transition lines mirror the behavior of the rotational temperature.

\section{Conclusions}

Compared to the vibrational relaxation probability of $\mathrm{NO}(v=3)$ scattered from RFMS1 and RFMS2 the vibrational relaxation probability of $\mathrm{NO}(v=11)$ scattered from RMFS3 is significantly increased. Yet, also in this case the observed vibrational relaxation is much lower compared to the vibrational relaxation of $\mathrm{NO}(v=11)$ scattered from gold or silver 41 . The reason for this is found in the fact that while $\mathrm{VO}_{2}(\mathrm{R})$ has a much higher electrical conductivity than $\mathrm{VO}_{2}(\mathrm{M})$ its metallic properties are far less pronounced compared to gold or silver. Therefore, it can not be fit into the picture of work function dependent vibrational relaxation probability shown in the article by Steinsiek ${ }^{[15}$ without compensation for the different electronic properties of $\mathrm{VO}_{2}$.

It is shown that the vibrational relaxation probability is slightly higher for $\mathrm{NO}(v=11)$ scattered from the high electrically conducting $\mathrm{VO}_{2}(\mathrm{R})$ compared to scattering from the low electrically conducting $\mathrm{VO}_{2}(\mathrm{M})$. The difference of vibrational relaxation probability between these two phases of $\mathrm{VO}_{2}$ is found to be in the range from $1 \%$ to $5 \%$.

The measured rotational temperatures of the $\gamma(4,11)$ transition band indicate direct scattering of $\mathrm{NO}(v=11)$ as they increase linearly with the translational energy of 
the incident molecular beam. As already seen with the rotational temperatures of $\mathrm{NO}(v=3)$ scattered from $\mathrm{VO}_{2}$ and $\mathrm{NO}(v=11)$ scattered from $\mathrm{SiO}_{2}$ the $\mathrm{NO}$ molecules in the relaxed vibrational state $v=10$ show much higher rotational excitation than the molecules that retain their initial vibrational excitation. This effect was not observed for NO scattering from $\mathrm{Au}(111)$ and $\mathrm{Ag}(111)$ surfaces 41 . 


\subsection{Discussion}

\subsubsection{Low Relaxation Probability of Scattered NO}

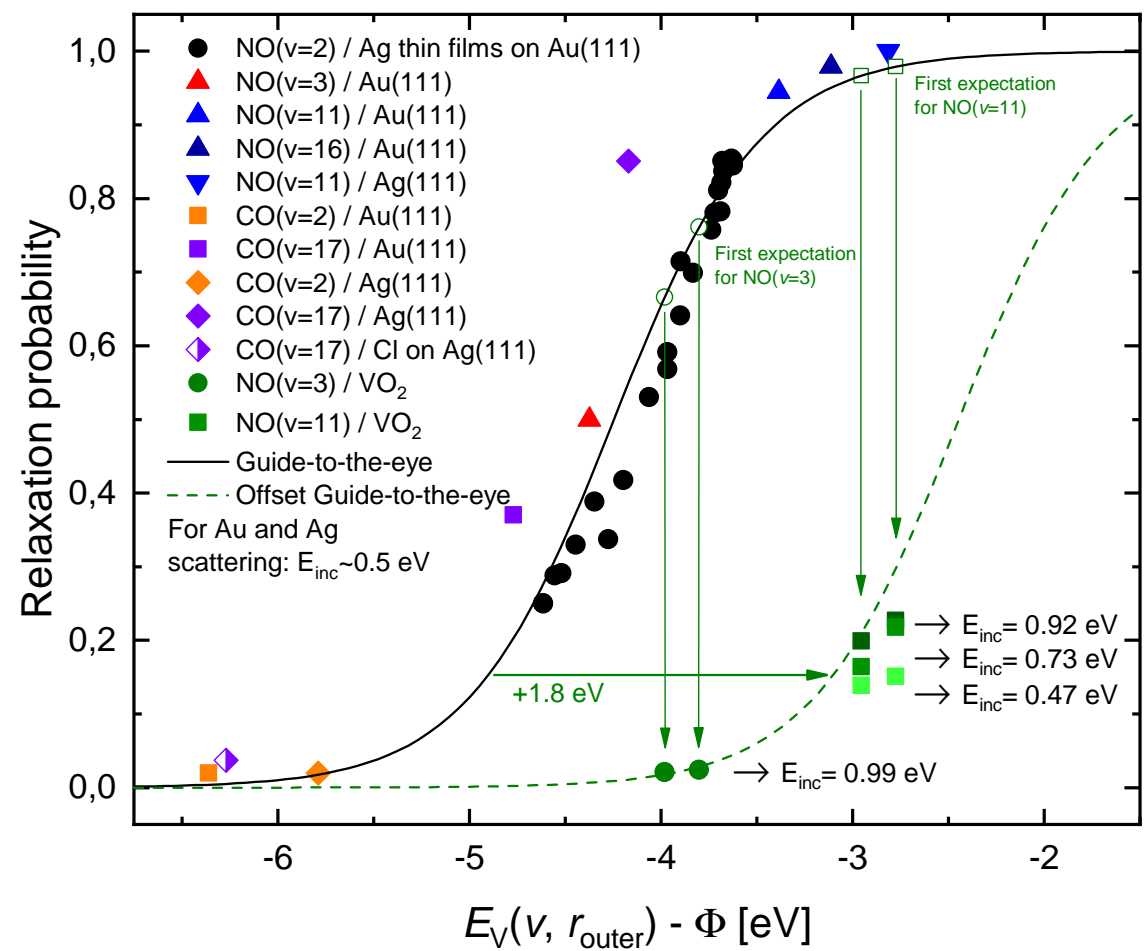

Figure 6.17: Graph of relaxation probability for vibrationally excited NO and CO from different metal surfaces as shown in Figure 2.13 with added points for $\mathrm{NO}(v=3)$ and $(v=11)$ scattered from $\mathrm{VO}_{2}$. The open circles are expectations for $v=3$ and $v=11$ scattered from $\mathrm{VO}_{2}$ based on the general trend seen from the previous experiments while the full points are the experimental results of this work. The green dashed line is the guide-to-the-eye line shifted to accommodate the results from scattering $\mathrm{NO}(v=3)$ from $\mathrm{VO}_{2}$.

The RFMS prepared $\mathrm{VO}_{2}$ samples are the first samples for which a clear correlation between the MIT of the sample and the vibrational relaxation probability of vibrationally excited NO molecules scattered from the sample surface are observed. This is done with a combination of the high order and flatness of the RFMS prepared $\mathrm{VO}_{2}$ thin films with its high MIT contrast and the application of low energy $\mathrm{O}_{2}{ }^{+}$sputtering that allows the removal of all detectable carbon contaminations while retaining most of the chemical integrity of the surface.

The resulting vibrational relaxation probabilities are compared to the previous experiments of NO scattered from gold and silver as shown in Figure 6.17. It can be easily seen that the vibrational relaxation probability of both $\mathrm{NO}(v=3)$ and $\mathrm{NO}(v=11)$ do 
not fit to the sigmoidal dependence between the vibrational relaxation probability and $\left|E_{\mathrm{v}}\left(v, r_{\text {outer }}\right)-\Phi\right|$ that was observed for NO scattering from $\mathrm{Ag}$ and $\mathrm{Au}$. 15]

Table 6.5: Vibrational relaxation probability of NO scattered from RFMS prepared $\mathrm{VO}_{2}$ thin films.

\begin{tabular}{cclc}
\hline vib. state of inc. NO & $\begin{array}{c}E_{\text {inc,trans }} \\
\text { in meV }\end{array}$ & surface & $\begin{array}{c}\text { vib. rel. probability } \\
\text { in \% }\end{array}$ \\
\hline$v=3$ & $986 \pm 7$ & RFMS1(M) & 2.1 \\
$v=3$ & $986 \pm 7$ & $\operatorname{RFMS1(R)}$ & 2.4 \\
$v=3$ & $986 \pm 7$ & $\operatorname{RFMS2(M)}$ & 2.1 \\
$v=3$ & $986 \pm 7$ & RFMS2(R) & 2.5 \\
$v=11$ & $920 \pm 80$ & RFMS3(M) & 20 \\
$v=11$ & $920 \pm 80$ & RFMS3(R) & 23 \\
$v=11$ & $730 \pm 40$ & RFMS3(M) & 16 \\
$v=11$ & $730 \pm 40$ & RFMS3(R) & 22 \\
$v=11$ & $470 \pm 30$ & RFMS3(M) & 14 \\
$v=11$ & $470 \pm 30$ & RFMS3(R) & 15 \\
\hline
\end{tabular}

The vibrational relaxation probabilities for all probed combinations of RFMS prepared $\mathrm{VO}_{2}$ thin films and vibrationally excited $\mathrm{NO}$ are listed in Table 6.5. Note that in the case of $\operatorname{NO}(v=11)$ scattering no transition bands of the vibrational states $v=0,1,2,3,4$ could be probed. Therefore, the relaxation probabilities of scattered $\mathrm{NO}(v=11)$ are potentially underestimated.

An explanation for the measured relaxation probabilities can be found in the fact that the relaxation probability is not only influenced by the molecule's vertical binding energy at the outer turning point of the vibration $E_{\mathrm{v}}\left(v, r_{\text {outer }}\right)$ and the surface's work function $\Phi$ but also by the image charge stabilization (ICS) of the surface. A larger ICS increases the stability of the $\mathrm{NO}^{-}$-ion close to the surface and therefore increases the time where a surface electron can be abstracted by the NO molecule. This is a vital step in the vibrational relaxation mechanism of scattered NO. The ICS can be described by the equation for the potential energy of a charged particle close to a dielectric surface that is found in the book by Griffiths ${ }^{[74}$ :

$$
V_{\mathrm{ICS}}=\left(\frac{1}{4 \pi \epsilon_{0}}\right)\left(\frac{\epsilon_{\mathrm{r}}-1}{\epsilon_{\mathrm{r}}+1}\right)\left(\frac{e^{2}}{4 z}\right) .
$$


$\epsilon_{0}$ is the free space permittivity, $\epsilon_{r}$ is the relative permittivity or dielectric constant, $e$ is the particle charge and $z$ is the distance between the particle and the surface. For a metal $\epsilon_{r}$ is infinite and therefore the term $\left(\frac{\epsilon_{\mathrm{r}}-1}{\epsilon_{\mathrm{r}}+1}\right)$ becomes 1 which reduces Equation 6.1 to

$$
V_{\mathrm{ICS}}\left(\epsilon_{r}=\infty\right)=\left(\frac{e^{2}}{16 \pi \epsilon_{0} z}\right) \text {. }
$$

The image charge stabilization $V_{\text {ICS }}$ is constant for both gold and silver. Therefore, taking it into account would just shift all points in Figure 6.17 by the same amount along the x-axis. For $\mathrm{VO}_{2}$ this is not the case. From an article by Yang ${ }^{[75]}$ the relative dielectric constant of $\mathrm{VO}_{2}$ is measured as $\epsilon_{r}\left(\mathrm{VO}_{2}(\mathrm{M})=36\right.$ below the MIT temperature and $\epsilon_{r}\left(\mathrm{VO}_{2}(\mathrm{R})=6.6 \times 10^{4}\right.$ above the MIT temperature. This results in corresponding ICS potentials of $V_{\mathrm{ICS}}\left(\epsilon_{r}=36\right)=0.95\left(\frac{e^{2}}{16 \pi \epsilon_{0} z}\right)$ and $V_{\mathrm{ICS}}\left(\epsilon_{r}=6.6 \times 10^{4}\right)=$ $1.00\left(\frac{e^{2}}{16 \pi \epsilon_{0} z}\right)$.

This shows that the difference in ICS of $5 \%$ between $\mathrm{VO}_{2}(\mathrm{M})$ and a metal is small. For $\mathrm{VO}_{2}(\mathrm{M})$ and a metal the difference is negligible. It is unlikely that this would have such a huge impact on the relaxation probability.

$V_{\text {ICS }}$ from Equation 6.1 is a function of $z$ which is described as the distance between charge and surface. But a more precise description would be that the ICS is dependent on the distance between the molecule and the image plane of the surface. The image plane is the centroid of the positive charge induced in the surface which does not necessarily has to be located at the surface and is different for different surfaces. Another formula for the ICS is used in an article by Wagner 13 . The article uses an equation postulated by Smith et. al ${ }^{76}$ which uses the distance between the image plane and the charge $\left(z-z_{0}\right)$ as a variable for the image charge stabilization:

$$
\begin{aligned}
& V_{\mathrm{ICS}}\left(z>z_{0}\right)=-\frac{1}{2\left(z-z_{0}\right)}\left(1-\exp \left(-\lambda\left(z-z_{0}\right)\right)\right) \\
& V_{\mathrm{ICS}}\left(z<z_{0}\right)=\frac{-U_{0}}{A\left(\exp \left(\beta\left(z-z_{0}\right)\right)\right)+1} .
\end{aligned}
$$

The parameter $\lambda, U_{0}, A$ and $\beta$ are given by Reference [76] for various metal surfaces including $\mathrm{Au}(111) . z_{0}$ is the distance of the image plane to the surface and $z$ the distance between the surface and the charge. With the corresponding parameters Equation 6.3 is reduced to

$$
\left.V_{\mathrm{ICS}}\left(z>z_{0}\right), \mathrm{Au}(111)\right)=-\frac{3.5985}{\left(z-z_{0}\right)} \cdot\left(1-\exp \left(-2.36 \cdot\left(z-z_{0}\right)\right)\right) .
$$

Equation 6.5 is plotted in Figure 6.18 with the position of $z=2 \AA$ and the corresponding ICS marked by dashed lines. According to Smith the image plane of $\mathrm{Au}(111)$ is located 


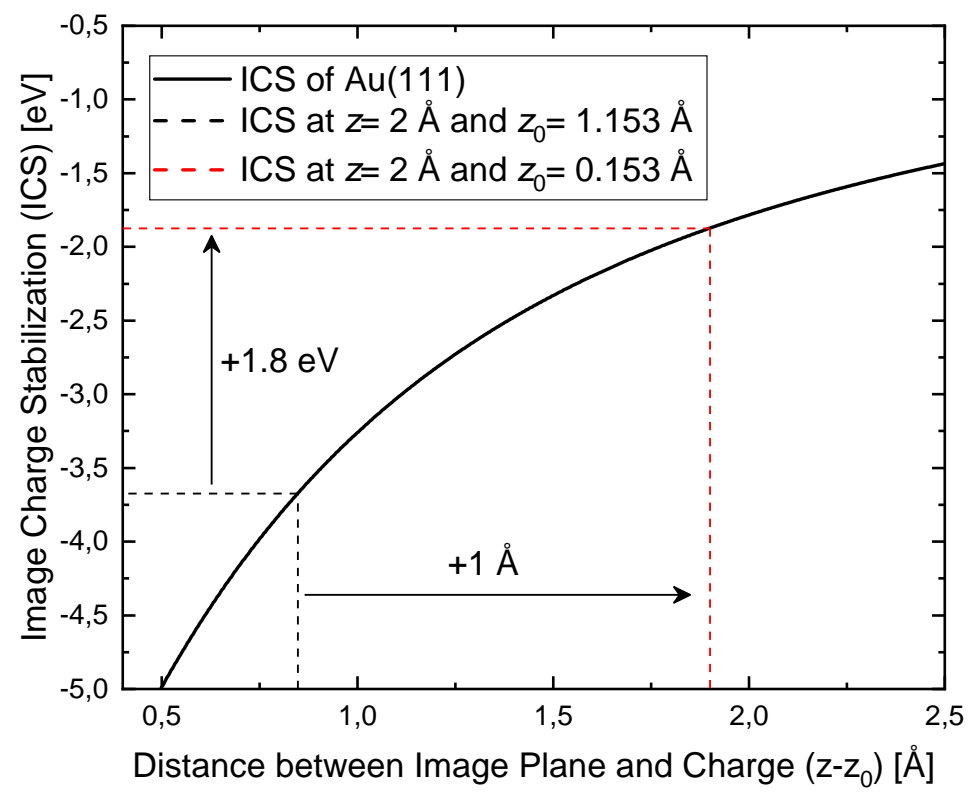

Figure 6.18: Image charge stabilization in dependence of of the distance between the NO molecule and the image plane for a $\mathrm{Au}(111)$ surface. The black dashed lines mark the ICS at $\left(z-z_{0}\right)=2 \AA$. According to Figure $2.15\left(z-z_{0}\right)=2 \AA$ is a position where the $\mathrm{NO}^{-}$-ion is stabilized enough for vibrational relaxation to occur. The ICS of $-3.7 \mathrm{eV}$ is according to the results shown in Figure 6.17 shifted by $+1.8 \mathrm{eV}$ to accommodate the ICS of $\mathrm{VO}_{2}$ (red dashed line). The resulting ICS of $-1.9 \mathrm{eV}$ is found on a $\mathrm{Au}(111) 1 \AA$ further away from the surface. From this it is deduced that to experience the same ICS a NO molecule has to approach a $\mathrm{VO}_{2}$ surface $1 \AA$ closer than a $\mathrm{Au}(111)$.

above the surface at $z_{0}=1.153 \AA$. The surface in this case is defined as "the outermost plane of nuclei" by Smith $[76$. By shifting the sigmoidal shown as a black line in Figure 6.17 along the $\mathrm{x}$-axis to match the points of vibrational relaxation probability of $\mathrm{NO}$ scattered from $\mathrm{VO}_{2}$ (green dashed line) one gains the difference in ICS between $\mathrm{VO}_{2}$ and gold and silver. The shift is in this case $1.8 \mathrm{eV}$ indicated by the horizontal green arrow.

The lower ICS reduces the stability of the $\mathrm{NO}^{-}$-ion and shifts its potential energy to higher values. In consequence, this reduces the probability of a non-adiabatic transition and subsequently the vibrational relaxation probability. According to Figure 6.18 a shift of $1.8 \mathrm{eV}$ of the ICS can be accomplished by shifting the image plane by about $1 \AA$ towards the surface. This means that according to the above stated assumption the image plane of $\mathrm{VO}_{2}$ is located about $0.1 \AA$ to $0.2 \AA$ above the surface. The number by itself appears to be reasonable but cannot be directly tested in the context of this work. 
Table 6.6: Charge carrier densities of $\mathrm{VO}_{2}$, gold and silver and corresponding work function $\Phi$ at room temperature and experimental vibrational relaxation probability of scattered $\mathrm{NO}(v=11) p_{\text {relax }, v=11}\left(E_{\text {trans,inc }} \approx 0.5 \mathrm{eV}\right)$.

\begin{tabular}{|c|c|c|c|}
\hline Compound & $n_{\mathrm{CCD}}\left[\mathrm{cm}^{-3}\right]$ & $\Phi[\mathrm{eV}]$ & $p_{\text {relax }, v=11}[\%]$ \\
\hline $\mathrm{VO}_{2}(\mathrm{M})$ & $7 \times 10^{14[75}$ & $4.88 \underline{60}$ & 14 \\
\hline $\mathrm{VO}_{2}(\mathrm{R})$ & $3 \times 10^{18[75]}$ & $4.70^{60}$ & 15 \\
\hline $\mathrm{Au}$ & $5.90 \times 10^{22[77}$ & $5.31^{78}$ & $94.5 \underline{41}$ \\
\hline $\mathrm{Ag}$ & $5.86 \times 10^{22[77}$ & $4.74^{78}$ & 10041 \\
\hline
\end{tabular}

Another approach to explain the difference in vibrational relaxation probability on NO scattered from $\mathrm{VO}_{2}$ and gold and silver is the difference in charge carrier density (CCD) between these materials. The CCD is the number of charge carriers per volume ${ }^{[79}$. For metals, all valence electrons supplied by the individual metal atoms count to charge carriers. The vibrational relaxation mechanism relies on the abstraction of an electron from the surface. A high CCD reduces the energy necessary for this to occur.

Table 6.6 lists the measured CCD of $\mathrm{VO}_{2}$, gold and silver. It can be seen that the CCD of both gold and silver is about $2 \times 10^{6}$ times higher than the $\mathrm{CCD}$ of $\mathrm{VO}_{2}(\mathrm{R})$. Ignoring the different work functions, this reduced CCD results in a significantly reduced relaxation probability of $\mathrm{NO}(v=11)$ scattered from $\mathrm{VO}_{2}(\mathrm{R})$. The difference in relaxation probability found is about $80 \%$. The $\mathrm{CCD}$ of $\mathrm{VO}_{2}(\mathrm{M})$ is furthermore about $4 \times 10^{3}$ smaller than the CCD of $\mathrm{VO}_{2}(\mathrm{R})$. Because in $\mathrm{VO}_{2}(\mathrm{M})$ the movement of the electrons is restricted, they can only partially be counted as charge carriers. This reduced CCD in $\mathrm{VO}_{2}(\mathrm{M})$ on the other hand is only accompanied by a minor yet noticeable reduction of the vibrational relaxation probability of scattered $\mathrm{NO}(v=11)$ by $1 \%$. From the low number of data points it is only possible to say that the vibrational relaxation probability of scattered NO increases with the CCD of the surface the NO is scattered from but not which principles it follows.

It should be noted that the ICS is a surface property and the CCD a bulk property. In consequence, only the former should have direct influence on the surface dynamics with vibrationally excited NO. It is reasonable to assume that the CCD influences the electronic structure of the surface and in consequence indirectly the surface dynamics but other influences such as the surface reconstructions have to also be taken into account. 


\subsubsection{Elevated Rotational Temperatures in Relaxed NO}

It is shown that the rotational temperature of the analyzed transition bands of NO scattered from $\mathrm{VO}_{2}$ is significantly higher than the temperature of the surface. This is an indicator for a transformation of incident translational energy to rotational excitation which is a phenomenon observed during direct scattering of NO from various surfaces. It is also found that the rotational excitation of the molecules that retain their initial vibrational state $(v=3$ or $v=11$ ) is significantly lower than the rotational excitation of molecules that relax to the next lower vibrational state ( $v=2$ or $v=10$, respectively) when scattered from $\mathrm{VO}_{2}$. The same effect is observed for $\mathrm{NO}(v=11)$ scattering from $\mathrm{SiO}_{2}$.

In the scattering experiments of $\mathrm{NO}(v=11)$ from $\mathrm{Au}(111)$ by Bastian Krüger 41 it is observed that the rotational excitation decreases with lower vibrational excitation for incident translational energies above $0.5 \mathrm{eV}$. This trend turns around at lower incident translational energies. A similar trend is observed by Kai Golibrzuch ${ }^{42}$ for $\mathrm{NO}(v=3)$ scattering from $\mathrm{Au}(111)$.

This is explained with a filtering effect that occurs during the scattering $41,73,80,81$. Depending on the orientation of the incident NO relative to the surface different rotational excitation in the scattered $\mathrm{NO}$ is observed. If the nitrogen atom faces the surface during collision, it results in scattered NO with a low average rotational energy and a high vibrational relaxation probability. If the oxygen atom faces the surface during collision it results in scattered NO with a higher average rotational energy and a low vibrational relaxation probability. In consequence, the vibrationally relaxed molecules are found rotationally colder than the molecules that retain their vibrational state during scattering.

Unfortunately, and in contrast to the $\mathrm{Au}(111)$ scattering experiments, only the rotational distributions of the two highest vibrational states $v=3$ and $v=2$ and $v=11$ and $v=10$ could be resolved so that a general trend along several vibrational states can not be observed. But since oxide surfaces such as $\mathrm{VO}_{2}$ and $\mathrm{SiO}_{2}$ have naturally little in common with the otherwise probed atomically-flat single crystal metal surfaces it can not be excluded that a different filtering mechanism occurs when scattering from oxide surfaces. It is also thinkable that during the scattering another mechanism is present that efficiently transfers vibrational energy to rotational energy. 



\section{Summary and Outlook}

In this work it is shown that the direct scattering channel is dominant when scattering NO from CVD prepared surfaces. Translational energies of all probed directly scattered NO molecules are obtained for CVD prepared surface with macroscopic roughness (CVD2 and CVD3). For one $\mathrm{VO}_{2}$ film with more severe defects (CVD1) the direct scattering component of the TOF distribution of NO in low $J$ states is overlaid with another scattering component. Here, the translational energies of directly scattered NO could only be partially probed. Therefore, scattering experiments under UHV are in principle not restricted to model systems such as $\mathrm{Au}(111)$ or $\mathrm{Ag}(111)$ but can also be done on materials that are closer to real life catalytic materials. An example for such a catalytic material is $\mathrm{V}_{2} \mathrm{O}_{5}$ as it is already used as a catalyst in the contact process and can be, like $\mathrm{VO}_{2}$, prepared with CVD.

In the context of the $\mathrm{NO}$ scattering experiments from $\mathrm{VO}_{2} \mathrm{O}_{2}{ }^{+}$-sputtering is established as a viable method for surface preparation. In contrast to $\mathrm{Ar}^{+}$-ion sputtering $\mathrm{O}_{2}{ }^{+}$-ion sputtering preserves the chemical composition of the $\mathrm{VO}_{2}$ film and in consequence reduces the loss of MIT contrast during sputtering. Furthermore, $\mathrm{O}_{2}{ }^{+}$-sputtering is shown to remove carbon contaminations much more efficiently than $\mathrm{Ar}^{+}$-sputtering.

By scattering vibrationally excited $\mathrm{NO}(v=3)$ and $\mathrm{NO}(v=11)$ from RFMS prepared surfaces it is shown that the vibrational relaxation probability of NO differs when it is scattered from $\mathrm{VO}_{2}(\mathrm{M})$ and $\mathrm{VO}_{2}(\mathrm{R})$. The vibrational relaxation probability of $\mathrm{NO}(v=3)$ scattered from $\mathrm{VO}_{2}$ is determined to be about $2 \%$ at $T_{\text {Surface }}<65^{\circ} \mathrm{C}$. It increases by $0.3 \%$ to $0.4 \%$ when the surface is heated to $T_{\text {Surface }}>71^{\circ} \mathrm{C}$. The vibrational relaxation probability of $\mathrm{NO}(v=11)$ scattered from $\mathrm{VO}_{2}$ is determined to be about $20 \%$ at $T_{\text {Surface }}<65^{\circ} \mathrm{C}$. It increases by about $3 \%$ when the surface is heated to $T_{\text {Surface }}>71^{\circ} \mathrm{C}$.

From comparison with previous experiments of vibrationally NO scattered from $\mathrm{Au}(111)$ and $\operatorname{Ag}(111)$ it is shown that the observed vibrational relaxation probabilities of NO scattered from $\mathrm{VO}_{2}$ thin films are significantly lower than the relaxation probabilities of NO scattered from metals. This is explained on one hand by the lower ICS of $\mathrm{VO}_{2}$ compared to gold and silver. From the results of this work a difference in ICS between gold and silver and $\mathrm{VO}_{2}$ of $1.8 \mathrm{eV}$ is deduced. The difference in vibrational relaxation 
probabilities can also be explained by the different $\mathrm{CCD}$ of gold and silver and $\mathrm{VO}_{2}$. Yet, from the available data it is not possible to analyze the exact relations between ICS, CCD and vibrational relaxation probability.

Table 7.1: Work functions and charge carrier densities for vanadium dioxide, silicon and germanium.

\begin{tabular}{|c|c|c|c|}
\hline Solid & Temperature $\left[{ }^{\circ} \mathrm{C}\right]$ & Work Function $[\mathrm{eV}]$ & $\mathrm{CCD}\left[\mathrm{cm}^{-3}\right]$ \\
\hline $\mathrm{VO}_{2}(\mathrm{M})$ & 300 & $4.88^{[60}$ & $7 \times 10^{14[75]}$ \\
\hline $\mathrm{VO}_{2}(\mathrm{R})$ & 350 & $4.70 \underline{60}$ & $3 \times 10^{18 \square 5}$ \\
\hline $\mathrm{Ge}$ & 300 & 4.7883 & $2.33 \times 10^{1384}$ \\
\hline $\mathrm{Ge}$ & 375 & $4.78^{83}$ & $7 \times 10^{14 \underline{84}}$ \\
\hline $\mathrm{Ge}$ & 900 & 4.78 & $3 \times 10^{1884}$ \\
\hline $\mathrm{Si}$ & 300 & $4.83^{85}$ & $9.65 \times 10^{986}$ \\
\hline $\mathrm{Si}$ & 500 & $4.83^{85}$ & $7 \times 10^{14 \underline{87}}$ \\
\hline $\mathrm{Si}$ & 1100 & $4.83^{85}$ & $3 \times 10^{1887}$ \\
\hline
\end{tabular}

Further scattering experiments with semiconductor surfaces might clarify the matter. Two candidates are germanium and silicon. Both are commercially available as cut single crystal surfaces. Their work functions fall close to the values found for $\mathrm{VO}_{2}$ as shown in Table 7.1. While their CCD at room temperature is significantly lower than the $\mathrm{CCD}$ of $\mathrm{VO}_{2}$ it scales with temperature so that at certain temperatures they reach comparable $\mathrm{CCD}$ values as found for $\mathrm{VO}_{2}(\mathrm{M})$ and $\mathrm{VO}_{2}(\mathrm{R})$. According to Reference 84] the $\mathrm{CCD}$ of Ge follows the empirical expression

$$
n_{\mathrm{CCD}}(\mathrm{Ge})=\sqrt{3.10 \cdot 10^{32} \cdot T^{3} \cdot \exp \left(\frac{-0.785}{k_{\mathrm{B}} \cdot T}\right)} .
$$

Here, $T$ is the temperature in $\mathrm{K}$ and $k_{\mathrm{B}}$ is the Boltzmann constant in $\mathrm{eV} \mathrm{K}^{-1}$. The temperatures where the CCD of Ge is equal to the CCD of $\mathrm{VO}_{2}(\mathrm{M})$ and $\mathrm{VO}_{2}(\mathrm{R})$ are listed in Table 7.1

The work function of Ge should only vary slightly between these two temperatures. By measuring vibrational relaxation probabilities of $\mathrm{NO}(v=3)$ and $\mathrm{NO}(v=11)$ scattered from a Ge surface at temperatures from $300 \mathrm{~K}$ to $1000 \mathrm{~K}$ it would be possible to probe a surface with a work function and CCD similar to $\mathrm{VO}_{2}(\mathrm{M})$ and $\mathrm{VO}_{2}(\mathrm{R})$. This way it can be tested if the relaxation probability of $\mathrm{NO}$ scales with work function or CCD. 


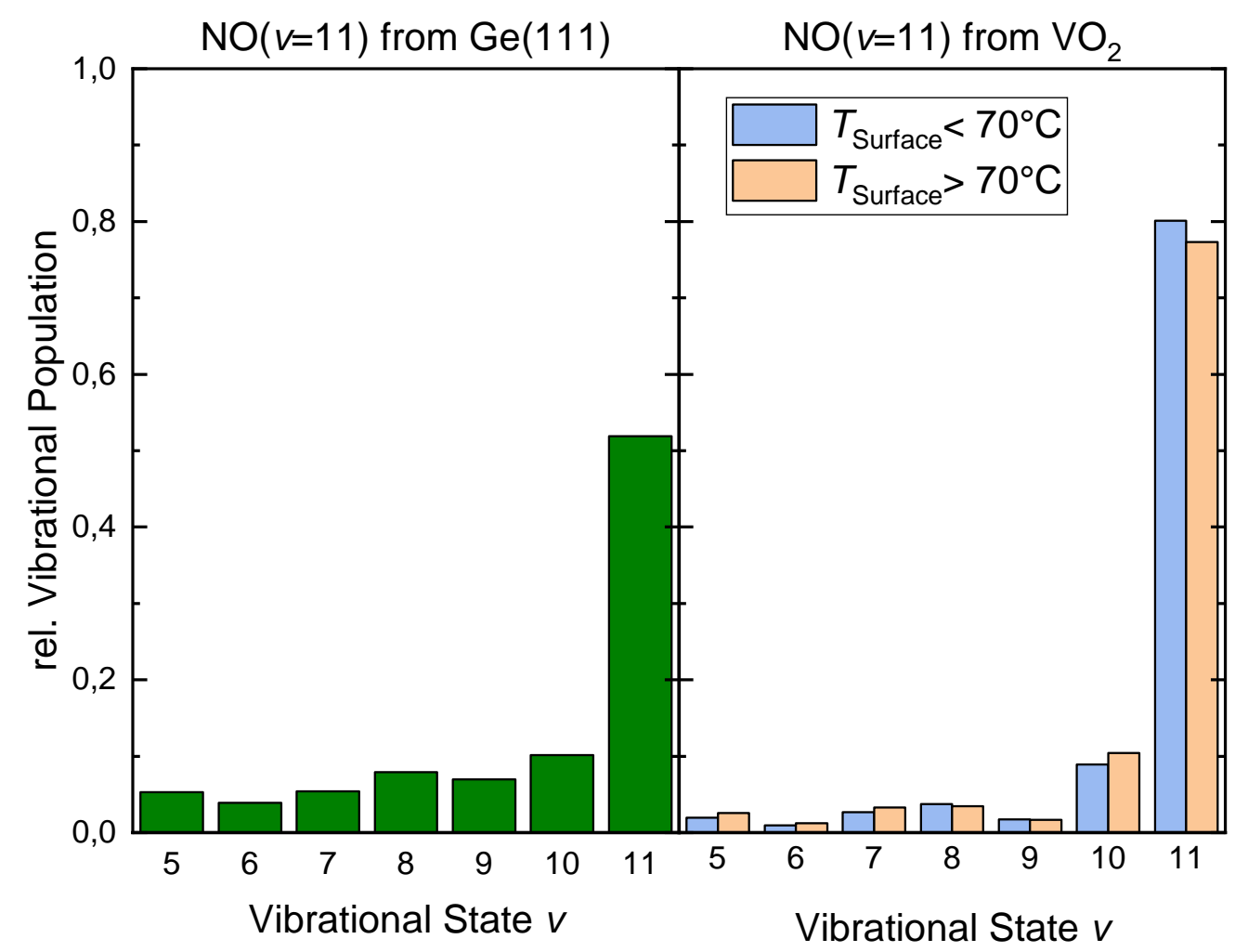

Figure 7.1: Vibrational state distributions of $\mathrm{NO}(v=11)$ scattered with $E_{\text {trans,inc }}=0.4 \mathrm{eV}$ from $\mathrm{Ge}(111)$ at $400{ }^{\circ} \mathrm{C}$ and $\mathrm{NO}(v=11)$ scattered with $E_{\text {trans,inc }}=0.9 \mathrm{eV}$ from $\mathrm{VO}_{2}$ around $70^{\circ} \mathrm{C} . \mathrm{Ge}(111)$ data is taken from Reference 88 .

In fact, NO scattering experiments with Ge have already been done but due to technical problems have been abandoned and the results have only been published in the $\mathrm{PhD}$ thesis of Bastian Krueger[1], the master thesis of Sven Meyer ${ }^{88}$ and the bachelor thesis of Niklas Henning $[99$. The vibrational state distribution from the only taken REMPI spectrum of $\mathrm{NO}(v=11)$ scattered from $\mathrm{Ge}(111)$ is shown with the results from this work in Figure 7.1. $\mathrm{Ge}(111)$ is held at $400^{\circ} \mathrm{C}$ because NO sticks to it at lower temperature. This makes it impossible to sustain a clean surface for more than a few minutes at lower temperatures when it is exposed to the molecular beam. Despite this constrain several temperatures and therefore CCDs of Ge can be probed between $400^{\circ} \mathrm{C}$ and its melting point.

The shown vibrational state distribution of $\mathrm{NO}(v=11)$ scattered from $\mathrm{Ge}(111)$ appears to have the same general structure as the vibrational state distribution of $\mathrm{NO}(v=11)$ scattered from $\mathrm{VO}_{2}$. The relaxation probability of about $48 \%$ of scattered $\mathrm{NO}(v=11)$ is significantly higher than the values found for $\mathrm{NO}(v=11)$ scattering from $\mathrm{VO}_{2}$. This 
is noteworthy because according to Equation 7.1 the $\mathrm{CCD}$ of $\mathrm{Ge}$ at $400^{\circ} \mathrm{C}$ should be about $4 \times 10^{17} \mathrm{~cm}^{-3}$ while the CCD of the probed $\mathrm{VO}_{2}(\mathrm{R})$ should be $3 \times 10^{18} \mathrm{~cm}^{-3}$. In general the scattering mechanism from $\mathrm{Ge}$ and $\mathrm{VO}_{2}$ appears to be comparable.

If NO scattering from Ge should not work, scattering experiments with a Si surface might serve as a substitute. Though, the CCD of Si is significantly smaller compared to $\mathrm{Ge}$ and $\mathrm{VO}_{2}$ it also increases with temperature ${ }^{87}$. It follows the empirical equation

$$
n_{\mathrm{CCD}}(\mathrm{Si})=2.8 \cdot 10^{16} \cdot T^{3 / 2} \cdot \exp \left(\frac{-6450}{T}\right)
$$

and can reach similar values as $\mathrm{VO}_{2}(\mathrm{R})$ at high temperatures. Temperatures where $\mathrm{Si}$ reaches the $\mathrm{CCD}$ of $\mathrm{VO}_{2}(\mathrm{M})$ and $\mathrm{VO}_{2}(\mathrm{R})$ are listed in Table 7.1. Si has also the advantage that its work function can be shifted by doping from $4.7 \mathrm{eV}$ to $4.9 \mathrm{eV}$ allowing for another adjustable experimental variable.

Additionally, to the observed vibrational state distributions also rotational state distributions of $\mathrm{NO}$ scattered from $\mathrm{VO}_{2}$ and $\mathrm{SiO}_{2}$ are analyzed for their rotational temperature and mean rotational energy. It is observed that in relaxed vibrational states such as $\mathrm{NO}(v=2)$ for $\mathrm{NO}\left(v_{\text {inc }}=3\right)$ and $\mathrm{NO}(v=10)$ for $\mathrm{NO}\left(v_{\text {inc }}=11\right)$ the rotational excitation is about $50 \%$ higher than in the initial vibrational state. This effect was not observed in NO scattering experiments from metal surface. Further experiments with $\mathrm{Ge}$ or $\mathrm{Si}$ might show if this is a general property of non-metals or is caused by the fact that both $\mathrm{VO}_{2}$ and $\mathrm{SiO}_{2}$ are oxides. 


\section{A Appendix}

\section{A.1 Custom-made LABView Environment}

To gather the data necessary for the work described above a custom LABView environment was written that combines all necessary tools to measure REMPI spectra, timeof-flight distributions and angular distributions. These are separated into accordingly labeled tabs located at the top of the front panel shown in Figure A.1. The described LABView program was used during the $\mathrm{NO}(v=11)$ scattering described in Section 6.2 . For the other experiments with $\mathrm{NO}(v=2)$ and $\mathrm{NO}(v=3)$ a predecessor program was used that is at its basis very similar to the program described in this section.

The program consists of a main loop that is supplied with processed data from three different secondary loops. The main loop is highlighted by a blue rectangle and labeled with (A) in Figure A.2. In this figure only the block diagram of the "Time-of-Flight" tab setting is shown. The block diagrams of the other cases of the tab selector "Setup", "Simple Spectral Scan" and "Fake Scan" are shown in Figure A.3. Figure A.4 and Figure A.5. The tab "Advanced Spectral Scan" is not explicitly shown because it only differs from "Simple Spectral Scan", shown in Figure A.4 by a feature to switch the REMPI laser timing. Exemplary the "Setup" and "Simple Spectral Scan" case's front panels are shown in Figure A.1. The "Setup" case is set as the default case where upfront of the measurement the integration borders for the MCP oscilloscope reading are set. These borders are separated in "Signal" (red) and "Background" (yellow) with a horizontal baseline (blue). All lines are set to position by dragging on the graph and applied with the "Set Window" button. When the program is closed the set integral borders and the background line positions are saved in a separate American Standard Code for Information Interchange (ASCII) file and used as default values when the program is started the next time.

The oscilloscope trace is read every $0.1 \mathrm{~s}$ and processed by the oscilloscope loop highlighted by the green rectangle labeled with (B) shown in Figure A.2. The oscilloscope trace with the integration borders is at all times shown in the bottom left corner of the front panel highlighted by the green rectangle in Figure A.1. The trace is offsetcorrected in y-direction with the value from the blue baseline. It is separated in a signal 


\section{A Appendix}

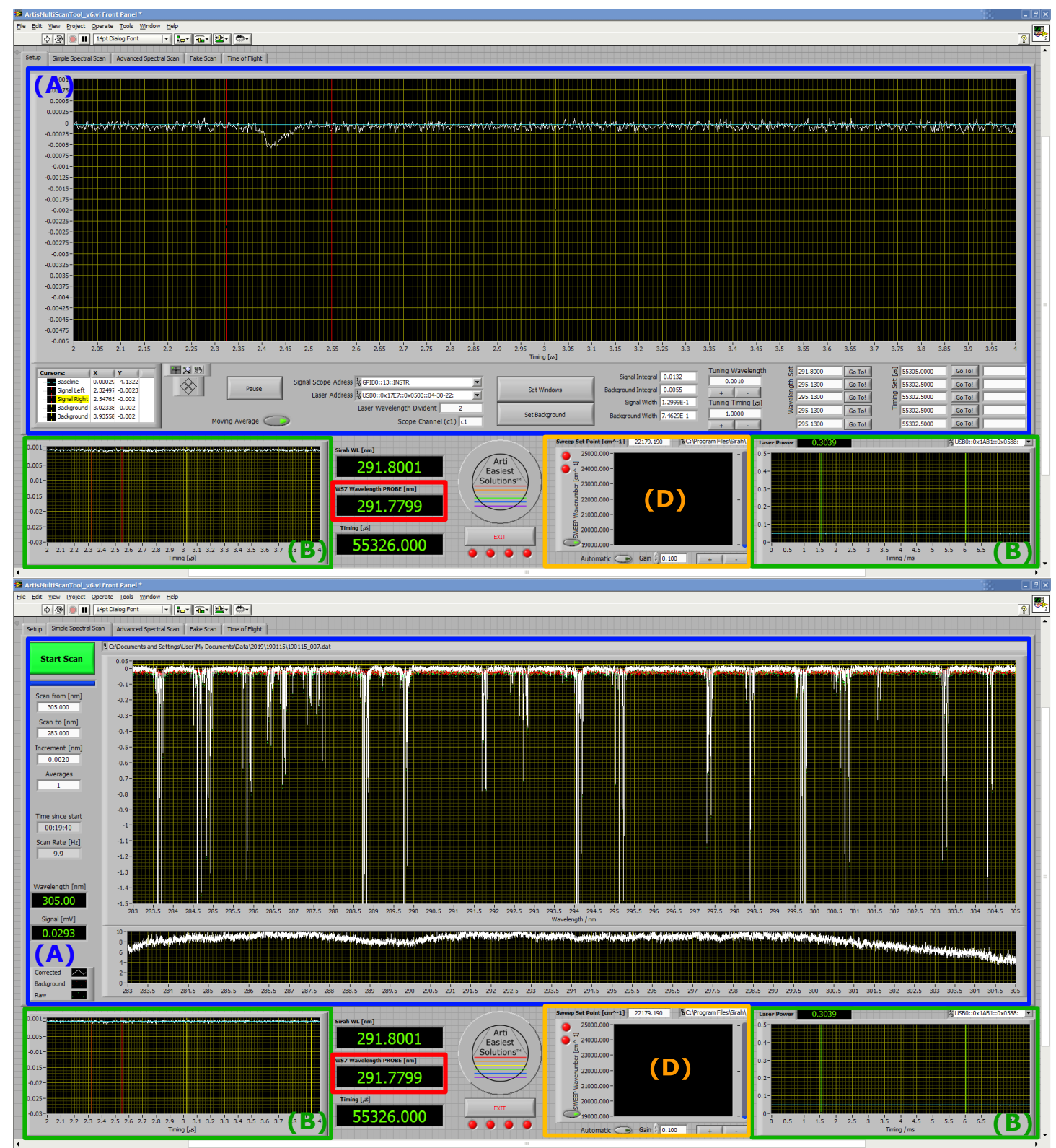

Figure A.1: Front panel of the Custom-made LABView Environment showing the "Setup" and "Simple Spectral Scan" tab. The colored rectangles correspond to the marked features in Figure A.2 The blue (A) rectangle shows the main screen which can be switched using the tab control. Here the measured data is shown and measurement parameters are set. The green (B) rectangle depicts oscilloscope readings. On the left side the MCP oscilloscope is shown with integration borders for signal and background. The red rectangle shows the wavemeter reading. The yellow (D) rectangle shows the reading and control of the SWEEP laser stabilization. 


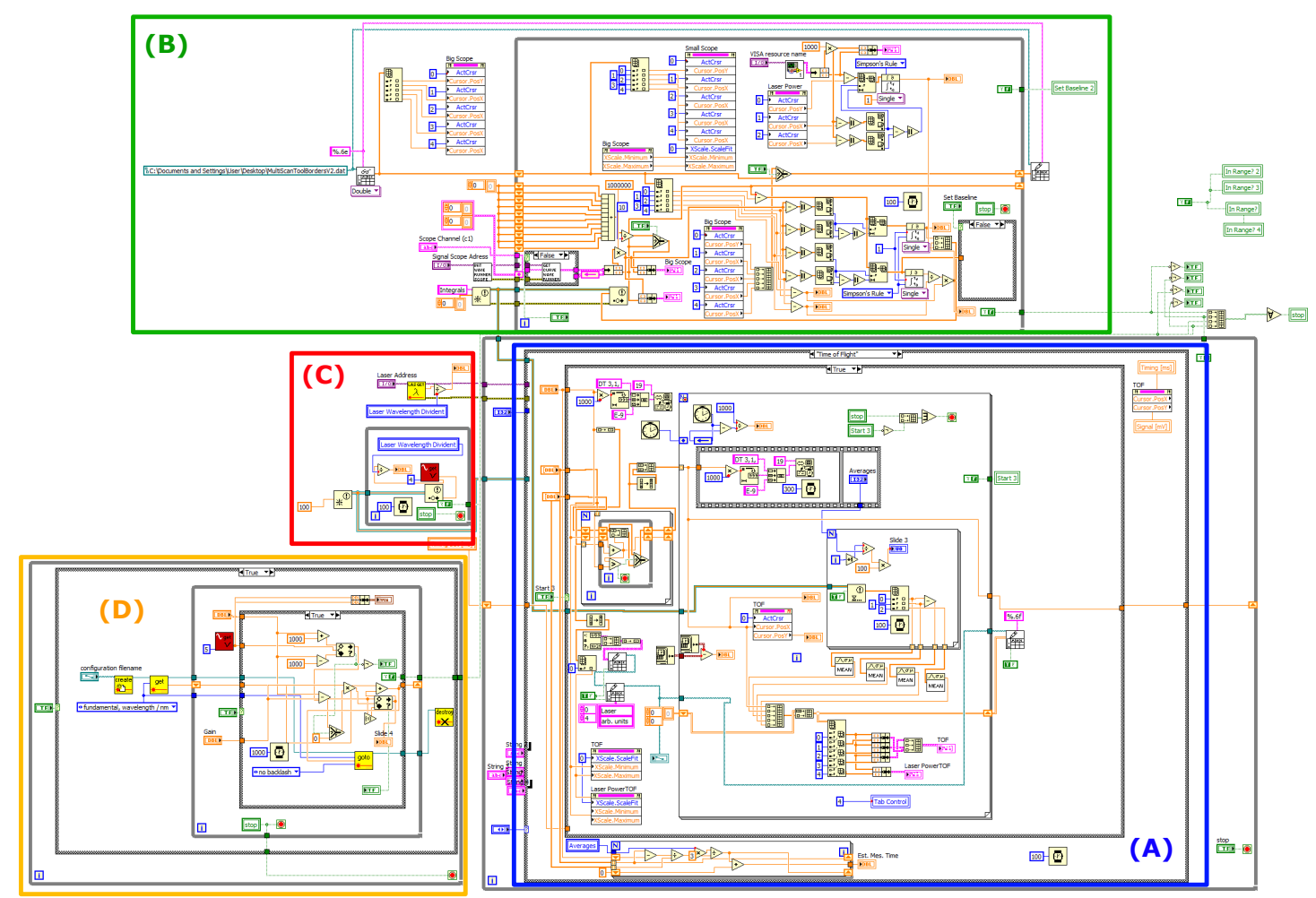

Figure A.2: Block diagram of the Custom-made LABView environment shown in Figure A.1. The diagram is separated in four sections that perform different tasks. The blue (A) rectangle highlights the main section of the program which controls the laser or delay generator scanning and combines the scanned points with the integrated oscilloscope reading into a visual graph. All other sections of the diagram direct data to the main section. Exemplary the "Time of Flight" tab is shown. The green (B) rectangle shows the while loop that repetitively reads the MCP and laser power oscilloscope and integrates the scope traces. The loop repetition is set to $10 \mathrm{~Hz}$ to match the laser repetition. The red (C) rectangle reads the laser frequency of the REMPI beam using the WS7 wave meter every $0.1 \mathrm{~s}$. The yellow (D) section reads and stabilizes the SWEEP laser wavelength by tuning the dye laser grid.

and background trace which are individually integrated using Simpson's Rule. The results of the signal and background integration and the difference between both is then provided to the main loop. In the XY graphs of the measurement tools the signal values are shown as green points, the background values as red points and the difference as white points as shown in the lower part of Figure A.1.

The oscilloscope loop also reads the trace of a second oscilloscope that is connected to a laser power measurement head onto which the REMPI laser beam is guided after it has crossed the UHV chamber. The oscilloscope trace and the integration borders are at all times shown in the bottom right corner of the front panel as shown in Figure A.1. 


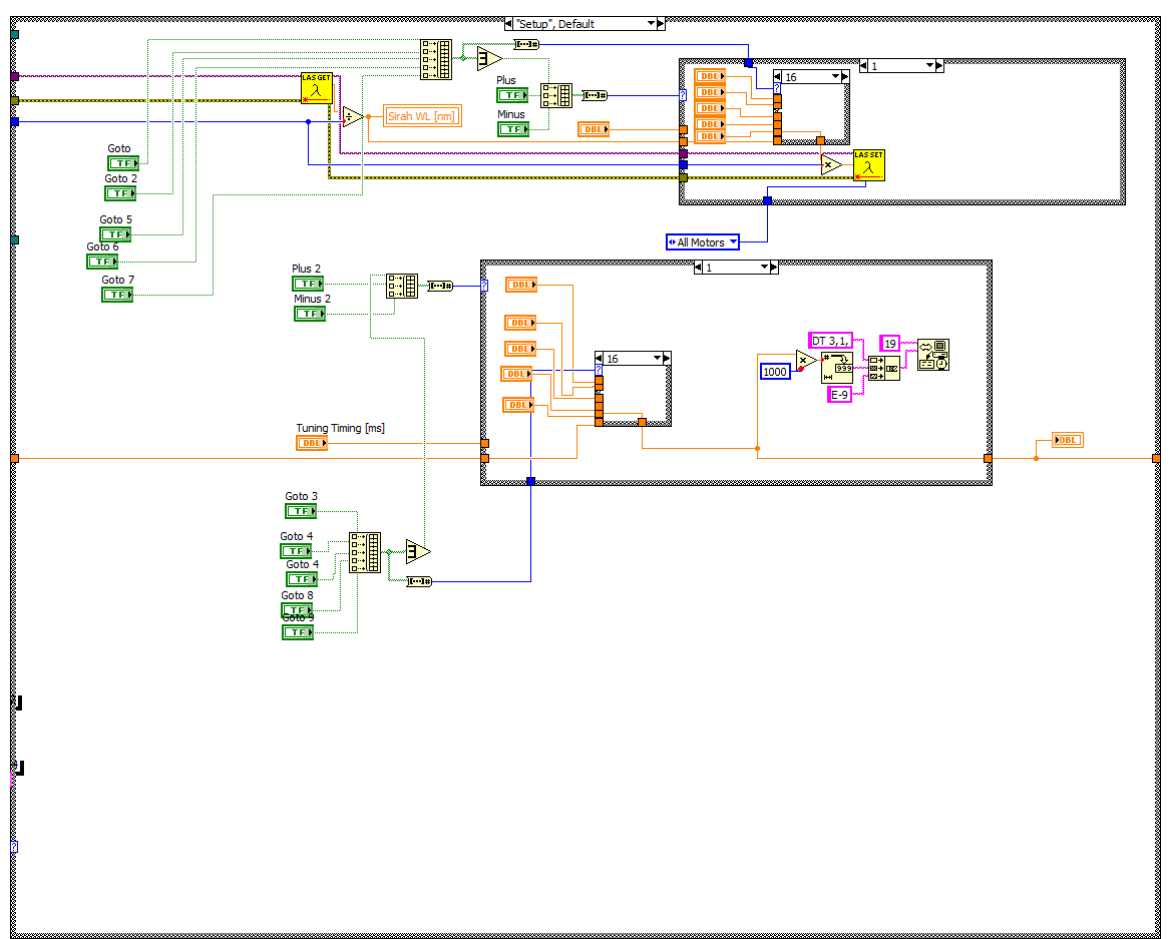

Figure A.3: Block diagram of the "Setup" case of the main loop highlighted by the blue (A) rectangle in Figure A.2 It used to define the integration borders for the MCP trace.

The integration value corresponds roughly to the REMPI laser power and is during the scan shown as a white line in a second XY graph below the measured data.

From the "Setup" panel both the setting of the REMPI delay generator and therefore the REMPI laser timing and the REMPI laser wavelength can be shifted or set to a specific point. The REMPI laser wavelength supplied by the intern grid calibration of the REMPI laser and the reading of the WS7 wavemeter (HighFinesse) (provided by the loop highlighted by the red rectangle labeled with (D) shown in Figure A.2 is shown at all times in the lower part of the front panel in green numbers on black ground. Below is the last set REMPI laser time delay shown in green numbers on black ground. The block diagram of the "Setup" case is shown in Figure A.3.

TOF distributions are measured using the "Time of Flight" tab which switches the main loop to the equally named case. The block diagram is shown in Figure A.2 highlighted by the blue rectangle. The TOF measurement starts with the program summarizing the set time delay intervals with individual step sizes to an array of time delays. The loop then goes through each time delay of the array. For each point the program first waits for $300 \mathrm{~ms}$ for the laser to settle before it takes a set number of points. The averaged points of the signal, background and difference integral are then plotted in the large 


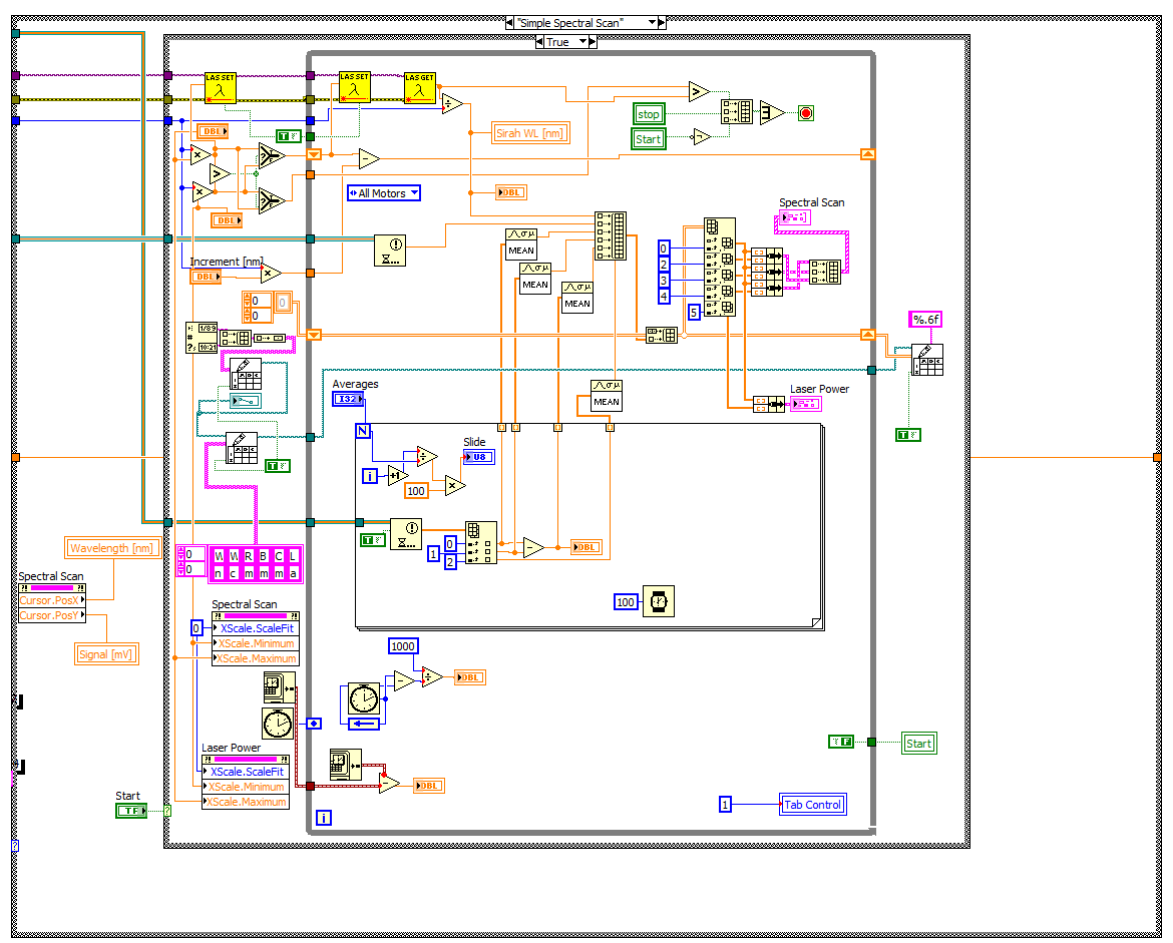

Figure A.4: Block diagram of the "Spectral Scan" case of the main loop highlighted by the blue (A) rectangle in Figure A.2. It is used to measure REMPI spectra.

XY graph with the corresponding REMPI laser power plotted in the smaller XY graph below.

The "Simple Spectral Scan" and "Advanced Spectral Scan" tabs are used to measure REMPI spectra by tuning the REMPI laser grid along a set spectral region. The block diagram of the "Simple Spectral Scan" case is shown in Figure A.4. The difference between "Simple Spectral Scan" and "Advanced Spectral Scan" is that the "Advanced" setting allows to change REMPI laser time delays during the scan at set positions of the spectrum. The wavelength tuning is done in set steps using the "Sirah Control 3.18" sub VIs supplied by "Sirah - Lasertechnik GmBH". For each wavelength a set amount of points is taken and averaged. The results are displayed on the large XY graph in different colors for "Signal", "Background" and "Difference". Like with the TOF described above during the scan the REMPI laser power of each point is simultaneously plotted in a smaller XY graph below the data points.

The "Fake Scan" tab works very similar to the other scanning tools described above but plots the measured points against time. This allows to measure changes in MCP signal over time when different properties not controlled by this program are scanned. One example are measurements where the surface temperature is ramped. Temperature 


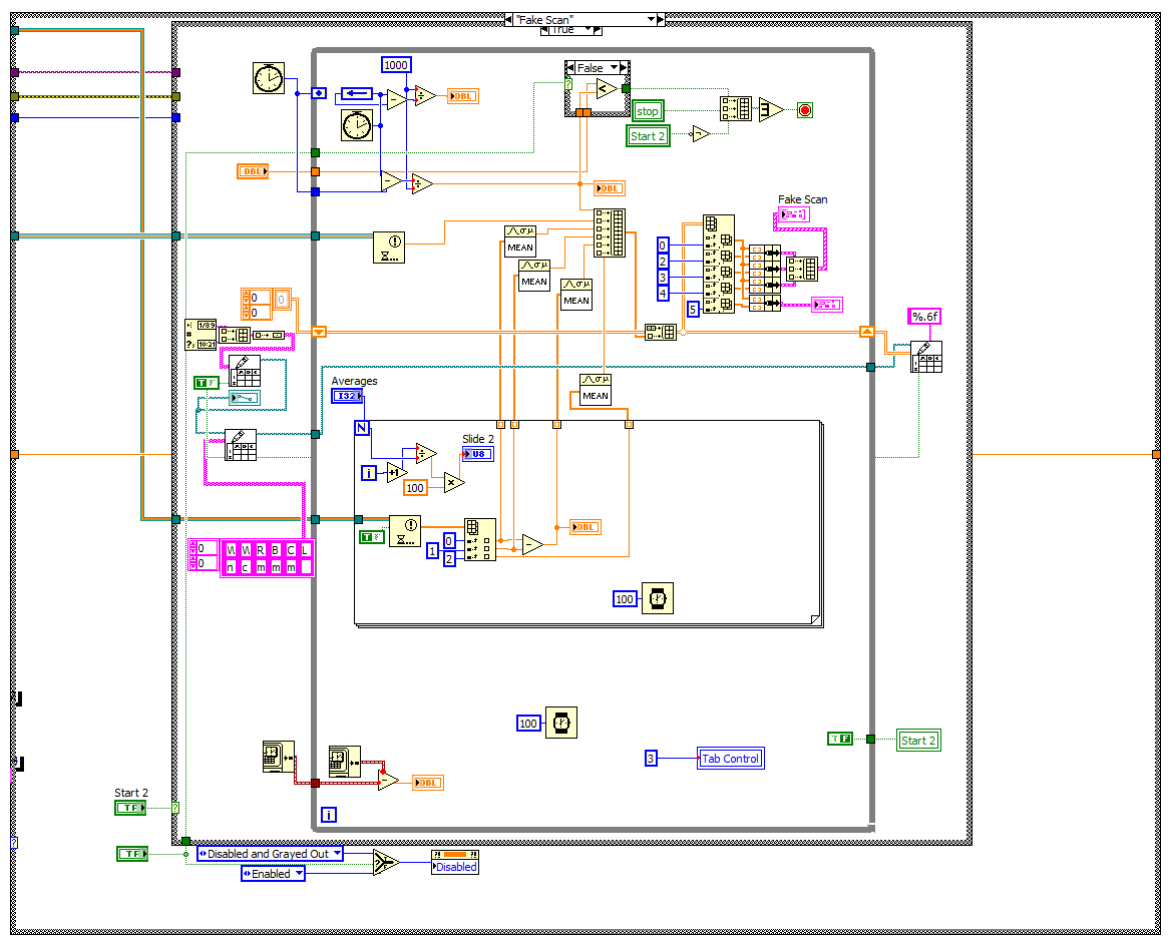

Figure A.5: Block diagram of the "Fake Scan" case of the main loop highlighted by the blue (A) rectangle in Figure A.2 It is used to measure the MCP signal over time.

control on this setup is done with a separate LABview program on a separate computer. In all cases the measured data is written as an ASCII file whose file path is always queried before the measurement starts. The file contains columns with the x-axis variable, the signal, background and difference integral value and the laser power in arbitrary units. The x-axis variable for "Time-of-Flight" is the time delay set by the program in us. The x-axis variable for the "Spectral Scans" and "Advanced Spectral Scan" tools is the wavelength in $\mathrm{nm}$ supplied by the REMPI dye laser and the wavenumber supplied by the wavemeter. The x-axis variable for the "Fake Scan" tab is the time in s.

The program is closed by pressing the large button labeled "EXIT" in the bottom of the front panel. It initiates all while-loops of the program to stop after their current cycle. This way it is prevented that an operation is canceled that can freeze the program. Each loop indicates its successful stop with a red boolean indicator below the "EXIT" button. 


\title{
List of Abbrevations and Indices
}

\author{
4-Probe four-terminal sensing \\ AES Auger-Meitner electron spectroscopy \\ $\mathrm{Ag} \quad$ silver \\ ASCII American Standard Code for Information Interchange \\ $\mathrm{Au} \quad$ gold \\ BBO $\quad \beta$-barium borate \\ CJC cold junction compensation \\ $\mathrm{Cl} \quad$ chlorine \\ CO carbon monoxide \\ cross crossing \\ CVD chemical vapor deposition \\ cw continuous wave \\ DFM difference frequency mixing \\ ds direct scattering \\ e electron \\ ECDL external cavity diode laser \\ el electronic \\ EHP Electron-hole pair \\ ESEM Environmental scanning electron microscope \\ FHG fourth harmonic generation \\ i irregular \\ inc incident \\ IR infra red \\ kin kinetic \\ $\mathrm{LiF} \quad$ lithium fluoride \\ $\mathrm{LiNbO}_{3}$ lithium niobate \\ MCP micro channel plate \\ MIT metal-to-insulator transition \\ MFC mass flow controller \\ Nd:YAG neodymium-doped yttrium aluminum garnet
}


NO nitric oxide

$\mathrm{O}_{2} \quad$ oxygen

OFHC oxygen free high conducting

OPA optical parametric amplification

PVD physical vapor deposition

QCM quartz micro balance

r regular

rot rotational

REMPI resonance enhanced multi-photon ionization

RGA residual gas analyzer

RFMS radio frequency magnetron sputtering

scat scattered

SEP stimulated emission pumping

SHG second harmonic generation

$\mathrm{Si} \quad$ silicon

$\mathrm{SiO}_{2} \quad$ silicon dioxide

subl sublimation

surf surface

TC thermocouple

td trapping desorption

TMP turbo molecular pump

TPD temperature programmed desorption

TOF time-of-flight

trans translation

$\mathrm{V}(\text { acac })_{2}$ vanadyl acetylacetonate

$\mathrm{VO}_{2} \quad$ vanadium(IV) oxide

$\mathrm{V}_{2} \mathrm{O}_{5} \quad$ vanadium(V) oxide

$\mathrm{VO}_{2}(\mathrm{M})$ monoclinic $\mathrm{VO}_{2}$

$\mathrm{VO}_{2}(\mathrm{R})$ rutile $\mathrm{VO}_{2}$

UHV ultra high vacuum

UV ultra violet 


\section{Bibliography}

[1] G. Wedler, Lehrbuch der physikalischen Chemie, Wiley-VCH, Weinheim, 2004.

[2] J. H. Sinfelt, Role of surface science in catalysis, Surface Science 2002, 500(1-3), 923-946, DOI 10.1016/s0039-6028(01)01532-1.

[3] W. G. Davenport, M. J. King, Sulfuric Acid Manufacture, Elsevier, 2006, DOI 10.1016/b978-0-08-044428-4.x5000-3.

[4] T. Kandemir, M. E. Schuster, A. Senyshyn, M. Behrens, R. Schlögl, The HaberBosch Process Revisited: On the Real Structure and Stability of Ammonia Iron under Working Conditions, Angewandte Chemie International Edition 2013, 52(48), 12723-12726, DOI 10.1002/anie.201305812.

[5] J. W. Erisman, M. A. Sutton, J. Galloway, Z. Klimont, W. Winiwarter, How a century of ammonia synthesis changed the world, Nature Geoscience 2008, 1, 636639 .

[6] I. Langmuir, The constitution and fundamental properties of solids and liquids. Part I Solids., Journal of the American Chemical Society 1916, 38(11), 2221-2295, DOI $10.1021 / \mathrm{ja0} 2268 \mathrm{a} 002$.

[7] A. K. Santra, D. W. Goodman, Catalytic oxidation of CO by platinum group metals: From ultrahigh vacuum to elevated pressures, Electrochimica Acta 2002, 47(22-23), 3595-3609, DOI 10.1016/s0013-4686(02)00330-4.

[8] J. Neugebohren, D. Borodin, H. W. Hahn, J. Altschäffel, A. Kandratsenka, D. J. Auerbach, C. T. Campbell, D. Schwarzer, D. J. Harding, A. M. Wodtke, T. N. Kitsopoulos, Velocity-resolved kinetics of site-specific carbon monoxide oxidation on platinum surfaces, Nature 2018, 558(7709), 280-283, DOI 10.1038/ s41586-018-0188-x.

[9] Y. Huang, Vibrational Promotion of Electron Transfer, Science 2000, 290(5489), 111-114, DOI 10.1126/science.290.5489.111. 
[10] K. Golibrzuch, N. Bartels, D. J. Auerbach, A. M. Wodtke, The Dynamics of Molecular Interactions and Chemical Reactions at Metal Surfaces: Testing the Foundations of Theory, Annual Review of Physical Chemistry 2015, 66(1), 399-425, DOI 10.1146/annurev-physchem-040214-121958.

[11] B. C. Krüger, N. Bartels, A. M. Wodtke, T. Schäfer, Final rotational state distributions from $\mathrm{NO}\left(v_{\mathrm{i}}=11\right)$ in collisions with $\mathrm{Au}(111)$ : the magnitude of vibrational energy transfer depends on orientation in molecule/surface collisions, Physical Chemistry Chemical Physics 2016, 18(22), 14976-14979, DOI 10.1039/c6cp02100j.

[12] B. C. Krüger, S. Meyer, A. Kandratsenka, A. M. Wodtke, T. Schäfer, Vibrational Inelasticity of Highly Vibrationally Excited $\mathrm{NO}$ on $\mathrm{Ag}(111)$, The Journal of Physical Chemistry Letters 2016, 7(3), 441-446, DOI 10.1021/acs.jpclett.5b02448.

[13] R. J. V. Wagner, N. Henning, B. C. Krüger, G. B. Park, J. Altschäffel, A. Kandratsenka, A. M. Wodtke, T. Schäfer, Vibrational Relaxation of Highly Vibrationally Excited CO Scattered from $\mathrm{Au}(111)$ : Evidence for CO Formation, The Journal of Physical Chemistry Letters 2017, 8(19), 4887-4892, DOI 10.1021/acs.jpclett. $7 \mathrm{~b} 02207$.

[14] R. J. V. Wagner, B. C. Krüger, G. B. Park, M. Wallrabe, A. M. Wodtke, T. Schäfer, Electron transfer mediates vibrational relaxation of $\mathrm{CO}$ in collisions with $\operatorname{Ag}(111)$, Physical Chemistry Chemical Physics 2019, 21 (4), 1650-1655, DOI $10.1039 / \mathrm{c} 8 \mathrm{cp} 06041 \mathrm{j}$.

[15] C. Steinsiek, P. R. Shirhatti, J. Geweke, C. Bartels, A. M. Wodtke, Work Function Dependence of Vibrational Relaxation Probabilities: $\mathrm{NO}(v=2)$ Scattering from Ultrathin Metallic Films of $\mathrm{Ag} / \mathrm{Au}(111)$, The Journal of Physical Chemistry $C$ 2018, 122(18), 10027-10033, DOI 10.1021/acs.jpcc.8b01950.

[16] G. Busca, L. Lietti, G. Ramis, F. Berti, Chemical and mechanistic aspects of the selective catalytic reduction of NO by ammonia over oxide catalysts: A review, Applied Catalysis B: Environmental 1998, 18(1-2), 1-36, DOI 10.1016/ s0926-3373(98)00040-x.

[17] C. Wu, F. Feng, Y. Xie, Design of vanadium oxide structures with controllable electrical properties for energy applications, Chemical Society Reviews 2013, 42(12), 5157, DOI 10.1039/c3cs35508j. 
[18] F. J. Morin, Oxides Which Show a Metal-to-Insulator Transition at the Neel Temperature, Physical Review Letters 1959, 3(1), 34-36, DOI 10.1103/physrevlett.3. 34.

[19] M. Imada, A. Fujimori, Y. Tokura, Metal-insulator transitions, Reviews of Modern Physics 1998, 70(4), 1039-1263, DOI 10.1103/revmodphys.70.1039.

[20] P. W. Atkins, Physical Chemistry, Wiley-VCH, 2001.

[21] J. H. de Boer, E. J. W. Verwey, Semi-conductors with partially and with completely filled 3d-lattice bands, Proceedings of the Physical Society 1937, 49(4S), 59-71, DOI 10.1088/0959-5309/49/4s/307.

[22] N. Mott, Metal-Insulator Transitions, CRC Press, 1990, DOI 10.1201/b12795.

[23] N. F. Mott, R. Peierls, Discussion of the paper by de Boer and Verwey, Proceedings of the Physical Society 1937, 49(4S), 72-73, DOI 10.1088/0959-5309/49/4s/308.

[24] J. C. Slater, Magnetic Effects and the Hartree-Fock Equation, Physical Review 1951, 82(4), 538-541, DOI 10.1103/physrev.82.538.

[25] N. N. Greenwood, A. Earnshaw, Chemistry of the elements, ButterwortHeinemann, 1997.

[26] U. Schwingenschlögl, V. Eyert, The vanadium Magnéli phases $\mathrm{V}_{\mathrm{n}} \mathrm{O}_{2 \mathrm{n}}-1$, Annalen der Physik 2004, 13(9), 475-510, DOI 10.1002/andp.200410099.

[27] M. Kang, I. Kim, S. W. Kim, J.-W. Ryu, H. Y. Park, Metal-insulator transition without structural phase transition in $\mathrm{V}_{2} \mathrm{O}_{5}$ film, Applied Physics Letters 2011, 98(13), 131907, DOI 10.1063/1.3571557.

[28] A. Heidemann, K. Kosuge, Y. Ueda, S. Kachi, Hyperfine interaction in $\mathrm{V}_{3} \mathrm{O}_{7}$, Physica Status Solidi (a) 1977, 39(1), K37-K40, DOI 10.1002/pssa.2210390152.

[29] K. Kosuge, T. Takada, S. Kachi, Phase Diagram and Magnetism of $\mathrm{V}_{2} \mathrm{O}_{3}-\mathrm{V}_{2} \mathrm{O}_{5}$ System, Journal of the Physical Society of Japan 1963, 18(2), 318-319, DOI 10.1143/jpsj.18.318.

[30] T. D. Manning, I. P. Parkin, M. E. Pemble, D. Sheel, D. Vernardou, Intelligent Window Coatings: Atmospheric Pressure Chemical Vapor Deposition of TungstenDoped Vanadium Dioxide, Chemistry of Materials 2004, 16(4), 744-749, DOI 10.1021/cm034905y. 
[31] T. J. Hanlon, J. A. Coath, M. A. Richardson, Molybdenum-doped vanadium dioxide coatings on glass produced by the aqueous sol-gel method, Thin Solid Films 2003, 436(2), 269-272, DOI 10.1016/s0040-6090(03)00602-3.

[32] W.-T. Liu, J. Cao, W. Fan, Z. Hao, M. C. Martin, Y. R. Shen, J. Wu, F. Wang, Intrinsic Optical Properties of Vanadium Dioxide near the Insulator-Metal Transition, Nano Letters 2011, 11 (2), 466-470, DOI 10.1021/nl1032205.

[33] D. Ruzmetov, G. Gopalakrishnan, C. Ko, V. Narayanamurti, S. Ramanathan, Three-terminal field effect devices utilizing thin film vanadium oxide as the channel layer, Journal of Applied Physics 2010, 107(11), 114516, DOI 10.1063/1.3408899.

[34] Z. Huang, C. Chen, C. Lv, S. Chen, Tungsten-doped vanadium dioxide thin films on borosilicate glass for smart window application, Journal of Alloys and Compounds 2013, 564, 158-161, DOI 10.1016/j.jallcom.2013.02.108.

[35] P. Kiri, M. E. A. Warwick, I. Ridley, R. Binions, Fluorine doped vanadium dioxide thin films for smart windows, Thin Solid Films 2011, 520(4), 1363-1366, DOI 10.1016/j.tsf.2011.01.401.

[36] A. Zylbersztejn, N. F. Mott, Metal-insulator transition in vanadium dioxide, Physical Review B 1975, 11(11), 4383-4395, DOI 10.1103/physrevb.11.4383.

[37] G. Herzberg, Molecular Spectra and Molecular Structure. Volume I: Spectra of Diatomic Molecules. Second Edition, D. Van Nostrand, 1950.

[38] J. M. Hollas, Modern Spectroscopy, John Wiley and Sons Ltd, 2003.

[39] P. Linstrom, NIST Chemistry WebBook, NIST Standard Reference Database 69, 1997, DOI 10.18434/T4D303.

[40] D. C. Jacobs, R. J. Madix, R. N. Zare, Reduction of $1+1$ resonance enhanced MPI spectra to population distributions: Application to the $\mathrm{NO} \mathrm{A}^{2} \Sigma^{+}-\mathrm{X}^{2} \Pi$ system, The Journal of Chemical Physics 1986, 85 (10), 5469-5479, DOI 10.1063/1.451557.

[41] B. Krüger, Dissertation, Georg-August University Goettingen, 2017.

[42] K. Golibrzuch, Dissertation, Georg-August-University Goettingen, 2014.

[43] N. Bartels, Dissertation, Georg-August University Goettingen, 2015.

[44] C. Amiot, R. Bacis, G. Guelachvili, Infrared study of the $\mathrm{X}^{2} \Pi(v=0,1,2)$ levels of ${ }^{14} \mathrm{~N}^{16} \mathrm{O}$. Preliminary results on the $v=0,1$ levels of ${ }^{14} \mathrm{~N}^{17} \mathrm{O},{ }^{14} \mathrm{~N}^{18} \mathrm{O}$, and ${ }^{15} \mathrm{~N}^{16} \mathrm{O}$, Canadian Journal of Physics 1978, 56(2), 251-265, DOI 10.1139/p78-032. 
[45] C. Amiot, J.-P. Maillard, J. Chauville, Fourier spectroscopy of the OD infrared spectrum. Merge of electronic, vibration-rotation, and microwave spectroscopic data, Journal of Molecular Spectroscopy 1981, 87(1), 196-218, DOI 10.1016/0022-2852(81)90089-8.

[46] C. Amiot, The infrared emission spectrum of NO: Analysis of the $\Delta v=3$ sequence up to $v=22$, Journal of Molecular Spectroscopy 1982, 94(1), 150 - 172, DOI https://doi.org/10.1016/0022-2852(82)90301-0.

[47] J. M. Brown, E. A. Colbourn, J. K. G. Watson, F. D. Wayne, An effective Hamiltonian for diatomic molecules, Journal of Molecular Spectroscopy 1979, $74(2)$, 294-318, DOI 10.1016/0022-2852(79)90059-6.

[48] M. J. L. de Lange, J. J. van Leuken, M. M. J. E. Drabbels, J. Bulthuis, J. G. Snijders, S. Stolte, Direct spectroscopic determination of the degree of orientation of parity-selected NO, Chemical Physics Letters 1998, 294(4-5), 332-338, DOI 10.1016/s0009-2614(98)00865-3.

[49] J. Danielak, U. Domin, R. Ke, M. Rytel, M. Zachwieja, Reinvestigation of the Emission $\gamma$ Band System $\left(\mathrm{A}^{2} \Sigma^{+}-\mathrm{X}^{2} \Pi\right)$ of the NO Molecule, Journal of Molecular Spectroscopy 1997, 181 (2), 394 - 402, DOI https://doi.org/10.1006/jmsp.1996. 7181.

[50] M. Barbato, C. Bruno, in Molecular Physics and Hypersonic Flows, Springer Netherlands, 1996, p. 139-160, DOI 10.1007/978-94-009-0267-1_8.

[51] D. N. Denzler, in Femtochemistry and Femtobiology, (Herausgegeben von J. S. Abderrazzak Douhal), World Scientific Publishing Co. Pte. Ltd., 2002, p. 653662 .

[52] J. C. Tully, in Dynamics of Molecular Collisions, Springer (US), 1976, p. 217-267, DOI 10.1007/978-1-4757-0644-4_5.

[53] J. C. Tully, Perspective on "Zur Quantentheorie der Molekeln", Theoretical Chemistry Accounts: Theory, Computation, and Modeling (Theoretica Chimica Acta) 2000, $103(3-4)$, 173-176, DOI 10.1007/s002149900049.

[54] E. Schrödinger, Quantisierung als Eigenwertproblem, Annalen der Physik 1926, 385(13), 437-490, DOI 10.1002/andp.19263851302.

[55] M. Born, R. Oppenheimer, Zur Quantentheorie der Molekeln, Annalen der Physik 1927, 389 (20), 457-484, DOI 10.1002/andp.19273892002. 
[56] H. S. W. Massey, Collisions between atoms and molecules at ordinary temperatures, Reports on Progress in Physics 1949, 12(1), 248-269, DOI 10.1088/0034-4885/12/ $1 / 311$.

[57] S. Roy, N. A. Shenvi, J. C. Tully, Model Hamiltonian for the interaction of NO with the $\mathrm{Au}(111)$ surface, The Journal of Chemical Physics 2009, 130(17), 174716, DOI 10.1063/1.3122989.

[58] R. Wagner, Dissertation, Georg-August University Goettingen, 2019.

[59] C. Steinsiek, Dissertation, Georg-August University Goettingen, 2017.

[60] A. Sohn, H. Kim, D.-W. Kim, C. Ko, S. Ramanathan, J. Park, G. Seo, B.-J. Kim, J.-H. Shin, H.-T. Kim, Evolution of local work function in epitaxial $\mathrm{VO}_{2}$ thin films spanning the metal-insulator transition, Applied Physics Letters 2012, 101(19), 191605, DOI 10.1063/1.4766292.

[61] C. Ko, Z. Yang, S. Ramanathan, Work Function of Vanadium Dioxide Thin Films Across the Metal-Insulator Transition and the Role of Surface Nonstoichiometry, ACS Applied Materials \& Interfaces 2011, 3(9), 3396-3401, DOI 10.1021/am2006299.

[62] C. Piccirillo, R. Binions, I. P. Parkin, Synthesis and Functional Properties of Vanadium Oxides: $\mathrm{V}_{2} \mathrm{O}_{3}, \mathrm{VO}_{2}$, and $\mathrm{V}_{2} \mathrm{O}_{5}$ Deposited on Glass by Aerosol-Assisted CVD, Chemical Vapor Deposition 2007, 13(4), 145-151, DOI 10.1002/cvde.200606540.

[63] T. D. Manning, I. P. Parkin, R. J. H. Clark, D. Sheel, M. E. Pemble, D. Vernadou, Intelligent window coatings: atmospheric pressure chemical vapour deposition of vanadium oxides, Journal of Materials Chemistry 2002, 12(10), 2936-2939, DOI $10.1039 / \mathrm{b} 205427 \mathrm{~m}$.

[64] T. Yamin, T. Havdala, A. Sharoni, Patterning of epitaxial $\mathrm{VO}_{2}$ microstructures by a high-temperature lift-off process, Materials Research Express 2014, 1(4), 046302, DOI 10.1088/2053-1591/1/4/046302.

[65] R. G. Musket, W. McLean, C. A. Colmenares, D. M. Makowiecki, W. J. Siekhaus, Preparation of atomically clean surfaces of selected elements: A review, Applications of Surface Science 1982, 10(2), 143-207, DOI 10.1016/0378-5963(82) 90142-8.

[66] L. E. Davis, Handbook of Auger Electron Spectroscopy, Physical Electronics Industries, Inc., 1976. 
[67] H. Hofsaess, P. Ehrhardt, H.-G. Gehrke, M. Broetzmann, U. Vetter, K. Zhang, J. Krauser, C. Trautmann, C. Ko, S. Ramanathan, Tuning the conductivity of vanadium dioxide films on silicon by swift heavy ion irradiation, AIP Advances 2011, 1 (3), 032168, DOI 10.1063/1.3646527.

[68] Q. Ran, D. Matsiev, A. M. Wodtke, D. J. Auerbach, An advanced molecule-surface scattering instrument for study of vibrational energy transfer in gas-solid collisions, Review of Scientific Instruments 2007, 78(10), 104104, DOI 10.1063/1.2796149.

[69] U. Even, J. Jortner, D. Noy, N. Lavie, C. Cossart-Magos, Cooling of large molecules below $1 \mathrm{~K}$ and He clusters formation, The Journal of Chemical Physics 2000, 112(18), 8068-8071, DOI 10.1063/1.481405.

[70] G. Scoles, Atomic and Molecular Beam Methods, Bd. 1, Oxford University Press, 1988.

[71] F. O. Goodman, Dynamics of gas-surface scattering, Elsevier, 2012.

[72] J. Luque, D. R. Crosley, in Database and Spectral Simulation.

[73] N. Bartels, K. Golibrzuch, C. Bartels, L. Chen, D. J. Auerbach, A. M. Wodtke, T. Schäfer, Dynamical steering in an electron transfer surface reaction: Oriented $\mathrm{NO}\left(v=3,0.08<E_{i}<0.89 \mathrm{eV}\right)$ relaxation in collisions with a $\mathrm{Au}(111)$ surface, The Journal of Chemical Physics 2014, $140(5), 054710$, DOI 10.1063/1.4863862.

[74] D. J. Griffiths, Introduction to Electrodynamics, Prentice Hall, 1999.

[75] Z. Yang, C. Ko, V. Balakrishnan, G. Gopalakrishnan, S. Ramanathan, Dielectric and carrier transport properties of vanadium dioxide thin films across the phase transition utilizing gated capacitor devices, Physical Review B 2010, 82(20), DOI 10.1103/physrevb.82.205101.

[76] N. V. Smith, C. T. Chen, M. Weinert, Distance of the image plane from metal surfaces, Physical Review B 1989, 40(11), 7565-7573, DOI 10.1103/physrevb.40. 7565 .

[77] A. Bansal, S. S. Verma, Searching for Alternative Plasmonic Materials for Specific Applications, Indian Journal of Materials Science 2014, 2014, 1-10, DOI 10.1155/ $2014 / 897125$.

[78] D. W. H. Rankin, CRC handbook of chemistry and physics, Crystallography Reviews 2009, 15(3), 223-224, DOI 10.1080/08893110902764125. 
[79] N. W. Ashcroft, N. Mermin, Solid State Physics, Cengage Learning, Inc, 1976.

[80] M. G. Tenner, F. H. Geuzebroek, E. W. Kuipers, A. E. Wiskerke, A. W. Kleyn, S. Stolte, A. Namiki, Orientation dependence of rotational excitation in NO scattering from $\mathrm{Ag}(111)$, Chemical Physics Letters 1990, 168(1), 45-50, DOI 10.1016/0009-2614(90)85100-q.

[81] N. Bartels, K. Golibrzuch, C. Bartels, L. Chen, D. J. Auerbach, A. M. Wodtke, T. Schafer, Observation of orientation-dependent electron transfer in moleculesurface collisions, Proceedings of the National Academy of Sciences 2013, 110(44), 17738-17743, DOI 10.1073/pnas.1312200110.

[82] A. F. Holleman, E. Wiberg, N. Wiberg, Lehrbuch der Anorganische Chemie, 102 edition, Walter de Gruyter, 2007.

[83] J. A. Dillon, H. E. Farnsworth, Work-Function Studies of Germanium Crystals Cleaned by Ion Bombardment, Journal of Applied Physics 1957, 28(2), 174-184, DOI 10.1063/1.1722701.

[84] F. J. Morin, J. P. Maita, Conductivity and Hall Effect in the Intrinsic Range of Germanium, Physical Review 1954, 94(6), 1525-1529, DOI 10.1103/physrev.94. 1525 .

[85] F. G. Allen, G. W. Gobeli, Work Function, Photoelectric Threshold, and Surface States of Atomically Clean Silicon, Physical Review 1962, 127(1), 150-158, DOI 10.1103/physrev.127.150.

[86] P. P. Altermatt, A. Schenk, F. Geelhaar, G. Heiser, Reassessment of the intrinsic carrier density in crystalline silicon in view of band-gap narrowing, Journal of Applied Physics 2003, 93(3), 1598-1604, DOI 10.1063/1.1529297.

[87] E. Conwell, Properties of Silicon and Germanium, Proceedings of the IRE 1952, 40(11), 1327-1337, DOI 10.1109/jrproc.1952.273956.

[88] S. Meyer, Master thesis, Georg-August-Universität Göttingen, 2015.

[89] N. Henning, Bachelor thesis, Georg-August-Universität Göttingen, 2014. 


\section{Acknowledgments}

I would like to thank Alec M. Wodtke and Igor Rahinov for the opportunity to write this thesis. I learned a lot from their experience and consider it an honor to work with them.

Furthermore, I would like to thank the whole Wodtke group and Rahinov group for their great support, the pleasant work environment and for all the times we had cake.

I would like to thank Anita who spend a lot of time in the lab making $\mathrm{VO}_{2}$ samples for instead of going to the beach.

Ich möchte mich besonders bei Tim Schäfer und Bastian Krüger bedanken für ihre stete Hilfe bei den Experimenten und der Auswertung und dafür, dass sie es sich auf sich genommen haben diese Arbeit korrekturzulesen.

Besonderen Dank möchte ich auch den Chemie-Werkstätten aussprechen, die immer gesagt haben, dass meine Entwürfe unmöglich umzusetzen sind. Zwei Tage später konnte ich die fertigen Teile dann meist abholen.

Ich möchte noch meinen Eltern danken, die immer alles in die Zukunft ihrer Kinder investiert haben. Egal, was es kostet und wie lange es dauern mag.

Zum Schluss möchte ich meiner Frau und besten Freundin Julia danken. Für ihre Unterstützung, wenn mir alles über den Kopf steigt und ihre aufmunternden Worte. Ohne dich hätte ich das nie zu Ende bringen können. 



\title{
Curriculum vitae
}

\author{
Artur Meling \\ Bergenstraße 17 \\ 37075 Göttingen \\ Day of birth: May 14th, 1989 \\ Place of birth: Kaskelen, Kazakhstan \\ Nationality: German \\ Marital Status: Married \\ Children: 1
}

\section{Education}

since Jun 2015

\author{
Graduate studies in physical chemistry \\ Georg-August-Universität Göttingen \\ Dissertation: Scattering of vibrationally excited NO \\ from vanadium dioxide \\ in the group of Prof. Dr. A. M. Wodtke
}

Okt 2012 - Apr 2015 Undergraduate studies in physical chemistry

Georg-August-Universität Göttingen

Master thesis: Preparation of Velocity Controlled Molecular Beams

of Highly Vibrationally Excited CO

in the group of Prof. Dr. A. M. Wodtke

Okt 2009 - Jul 2012 Undergraduate studies in physical chemistry

Georg-August-Universität Göttingen

Bachelor thesis: Soft landing of Ta nanoparticles on various substrates in the group of Prof. Dr. A. M. Wodtke

Aug 2002 - Apr 2008 Grammar school

Johannes-Kepler-Gymnasium Garbsen

Degree: Abitur 


\section{Miscellaneous}

Jul 2008 - Mar 2009 Military Service

Obergefreiter at the 4./Panzerartillerielehrbataillon 325

in Munster

Okt 2013 - Okt 2014 Dormitory spokesman

Student dormitory "Kellnerweg"

in Göttingen 